\title{
MBsthK
}


hor or y

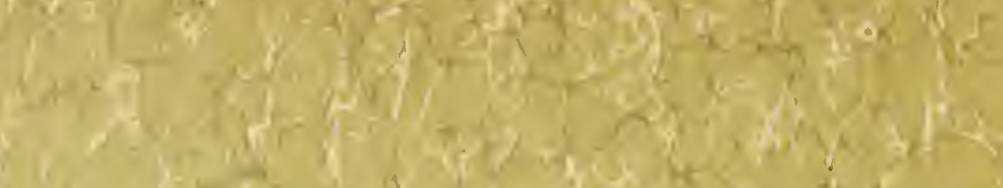

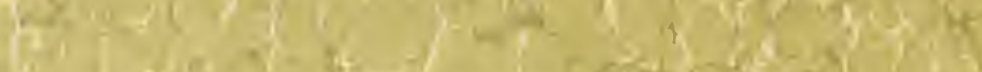

exint

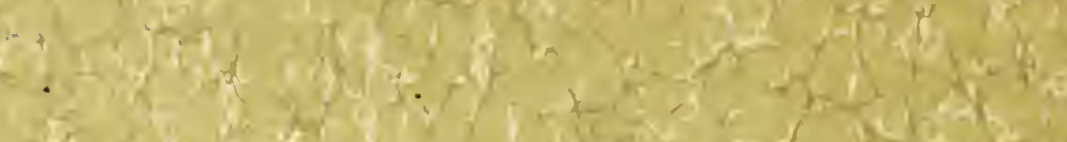

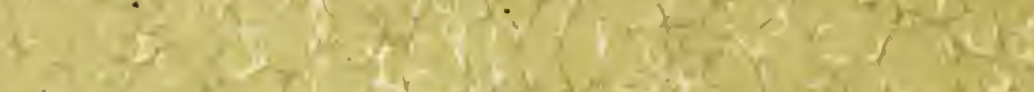
(15x) $4 x^{2} x^{2}$ and

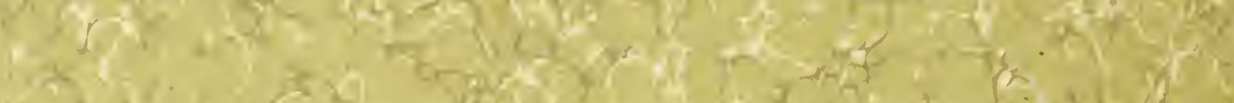

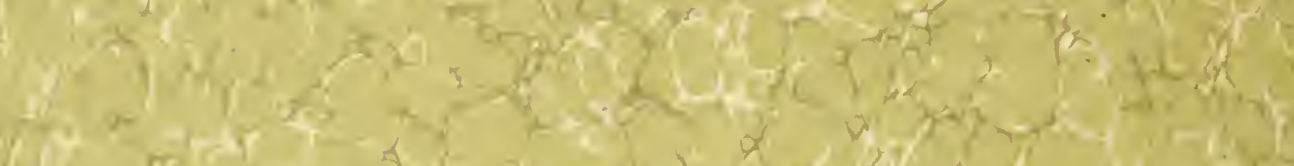

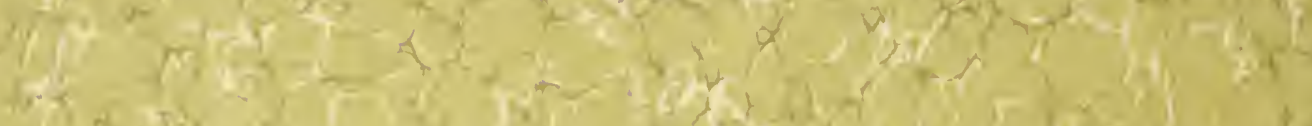

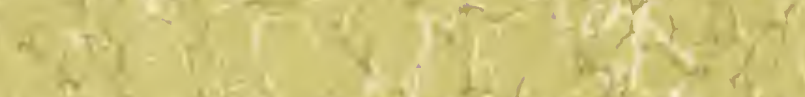

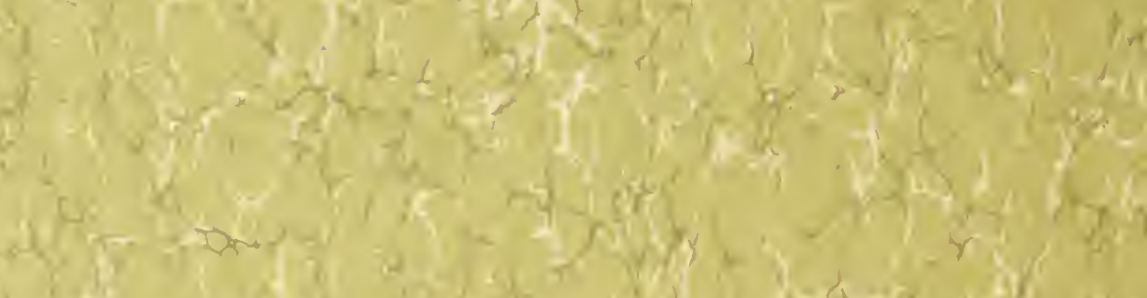

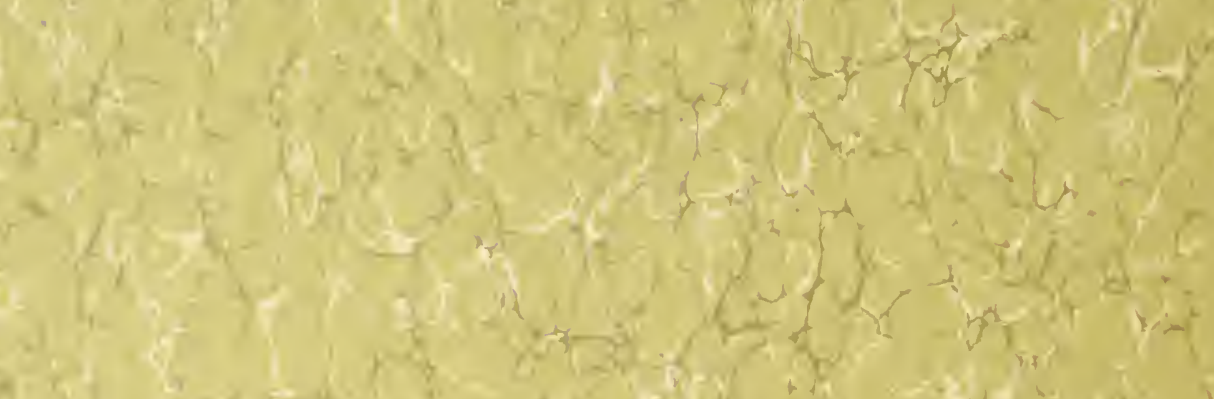
Q

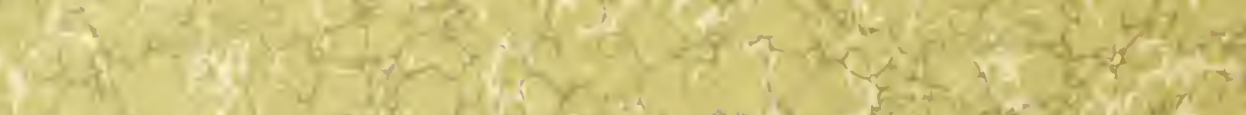

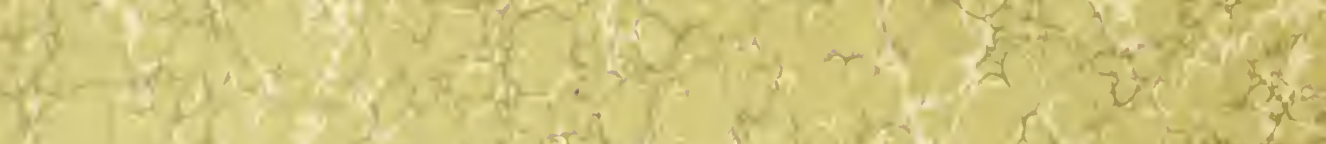

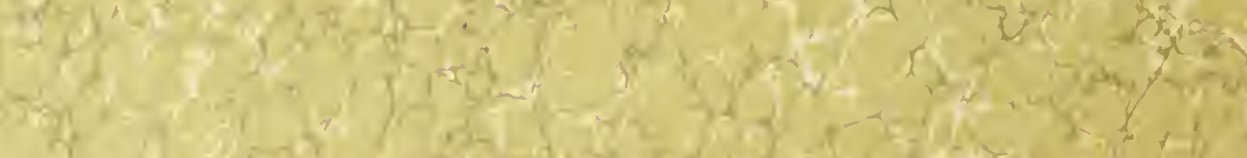

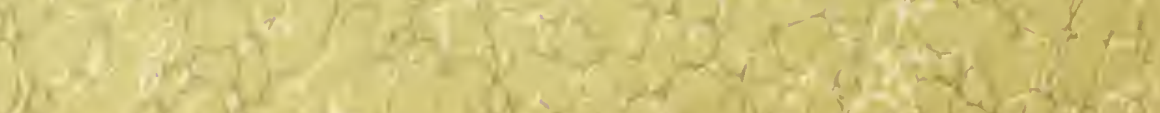




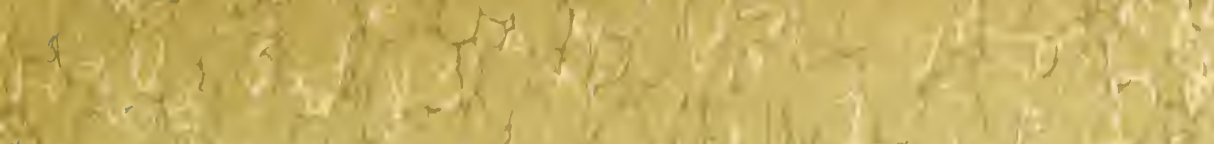

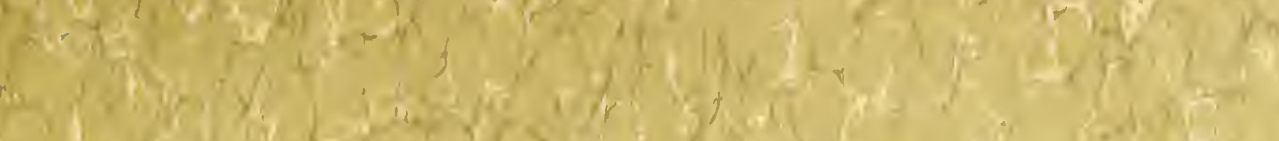

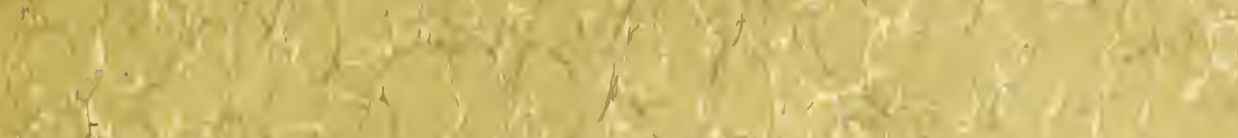

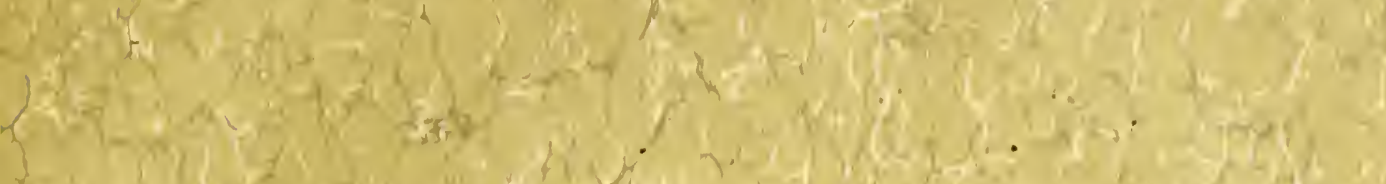

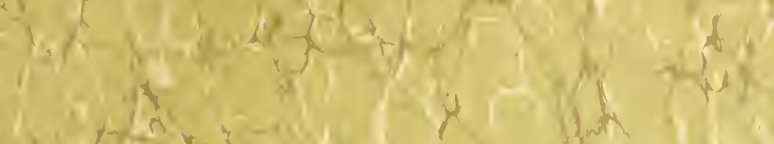

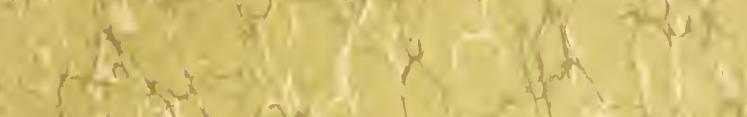

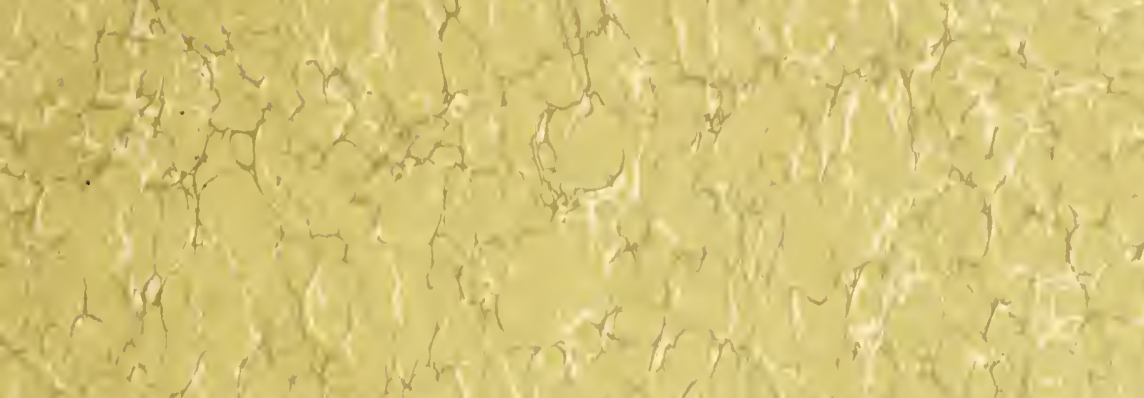

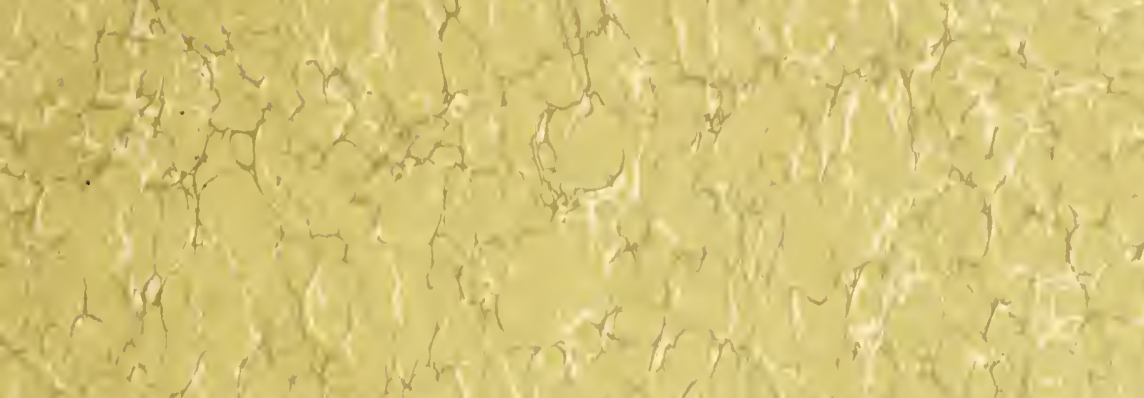

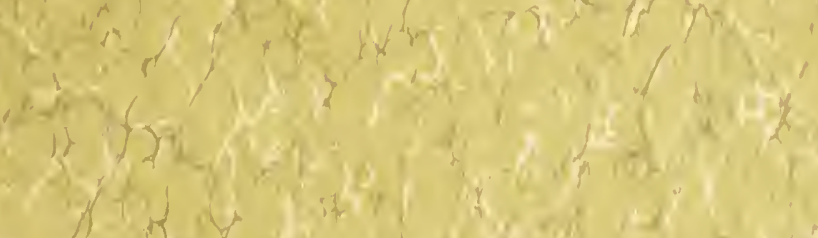
Sol

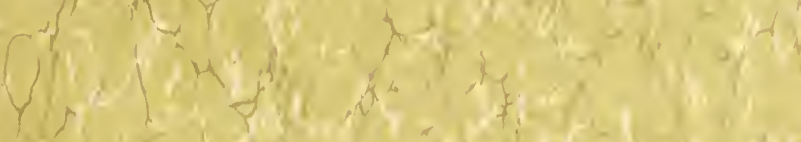
1)

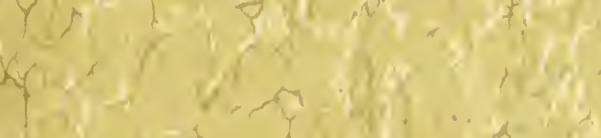

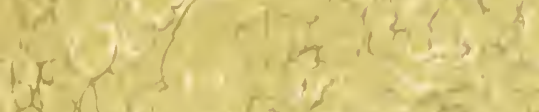
सhy

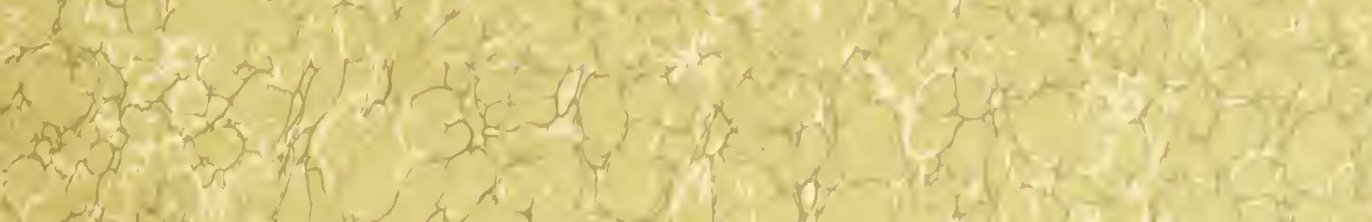

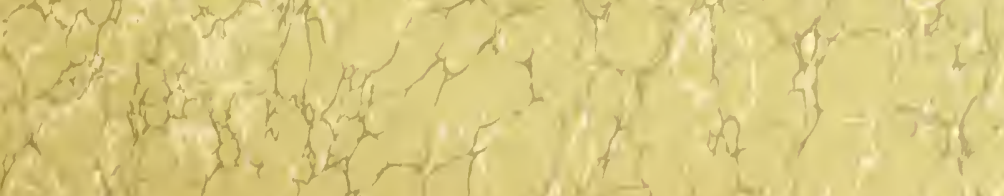



ACTA SOCIETATIS SCIENTIARUM FENNICE

TOM. XXXIV. № 1 .

\section{LE CONTE}

DE LA

\section{FEMME CHASTE CONVOTÉE PAR SON BEAU-FRERL}

\section{ÉTUDE DE LITTÉRATURE COMPARÉE}

PAK

A. WALLENSKÖLD

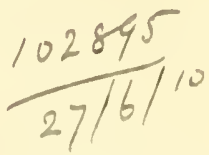




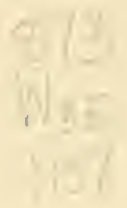

A MES CHERES COLLABORATRICES

$$
\begin{gathered}
M^{n E} \text { DAGMAR WALLFNSKÖLD } \\
M^{\text {LLJ VALBORG WALLENSKÖLD }}
\end{gathered}
$$

A W 


\section{CHAPITRE I}

\section{Classement général des différentes versions}

La condamnation imméritée d'une femme accusée d'adultère et sa réluabilitation après une longue suite d'aventures malhemrenses est un sujet légendaire sourent utilisé et qui a tronvé sa plus célèbre expression dans la légende de Geneviève de Brabant. ${ }^{1}$ Ce qui caractérise le conte dont nous nous occuperons dans cette étude, ce sont deux traits communs à tontes ses versions principales: 1) le premier (et, dans quelques versions, le seul) amant rebuté est le frère du mari, et 2) les persécuteurs de liérö̈ne (ou, dans quelques versions, le seul persécuteur, le bean-frère), chutiés par des maladies, sont guéris par leur victime elle-même. après qu'ils ont confessé leurs méfaits.

1 Dans son ceuvre magistrale, Manmorks gamle Folkeviser, Srend Grundtrig, partant de la ballade danoise Ravengaard og Memering, traita des divers groupes de légendes basées sur ce thème (v. t 1 [1853], p1. 177-213). Depuis, on a pu compléter son exposé systématique; v. notanment, sur la fille sans mains. H. Suchier, (Euvres poétiques de Philippe de Remi, Sire de Beaumanoir, t. I (1854), pp. XXIII-LXXXI; sur la femme persécutée qui perd ses deux fils, P. Streve, Die Ortavian-Sage (1544); sur la légende de Gcneviève de Brabant, B. Golz, Pfalzgräfin Genovefa in der deulschen Dichtung (1597); sur l'histoire d'un faux amant introduit dans le lit de la fomme persécutée, (i. Paris, Le Roman du Comte de Toulouse, 1). 12, note 1 (extrait des Annales du Vidi, t. XII $[\mathbf{1 9 0 0}\})$; etc. etc. Ajontons à la liste des contes ayant pour sujet les aventures d'une femme injustement accusée par un ou plusieurs amauts repoussés, les contes de provenance orientale suivants:

1) Hisloire de la fille vertucuse, dans G. Spitta-Bey, Contes arabes modernes (1883), Pp. 80-93 (no. VI),

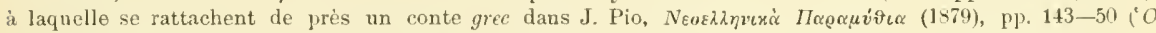

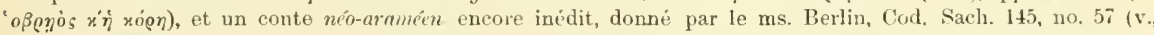
sur ce ms., M. Lidzbarski, Geschielten und Lieder aus den new-aramäisrhen Hss. der Kgl. Bibl. zu Berlin [1596], p. 139; le conte en question est à tort rattachè à notre conte oưr. cité, p. 171. 11. F. Fuchs, de Berlin, a bien voulu nous en donner nne traduction allemande), ainsi que, avec plus de divergences, un conte russe dans A. N. Alauasieff, Народныа Русскія Сказк. t. VII (1863), pp. 12-24 (Волпебие зеркалье [Le petit miroir enckrntéf). où il y a mélange avec le thème du conte de Blanche-Neige, et un conte fimnois dans K. Krrohn et L. Lilius, Sumalaisia Kunsansatuja, 2 osa: Funinkaallisia saluja, 1 vihkn (Tietecllinen painos) [Contes populaires finnais, 2:e partie: Cuntes royaux, lier fasc. (Ed. scientifique)] (1593), pp. 14-17 (No. 3: hauppiaan tytär [La fille du marchand]) et 149-52 (variantes sous la rubriøue: Tiattomasti syytetty neiti [La fille injustement accusée]).

2) Le Roi Bahrîm, ses deux Vizirs Kihássa é Khalássa et la fille du premier, dans le Tauti-Xameh de Nakhchabi, nuit 51 (v. la traduetion allemande de Pertsch, Zeitselur. der Deutsrhen morgenl. Gesellshaft. t. XXI [1567], pp. 543-50). Apparenté de près ì ce conte persan est un conto du Balihtyar-Simeh, Le Roi Dîdbin it ses cleux Vizirs (v., pour la version persane, Ouseley-Clouston, The Bahbtyar Nrima [1983], pp. 62-72, et W. A. Cloustou dans Originals and Anulayzes of some af Chancer's Cantertnerg Trtes [14rit, pp. 390-15; poux 
('e conte, que nous désignerons du nom de conte de lu femme chaste convoite pur som benu-frère, fante d'mn nom propre rommun i plusiem's gronpes de versions ${ }^{2}$. a déji depnis longtemps attiré l'attention des savants. J. C'. 'l'n. Girässe fut le premier' чlui, dans son grand onvage très nomri. mais farfois inexact, Die grossen sugenkreise des Mittrlulters (1S42), essaya de dresser me liste des différentes versions ("Florentia von lom" $)^{2}$. Son exposé est très incomplet ef he distingne pas assez nettement notre conte d'antres contes d'origine différente. Trois ans après, P. O. Bäckströ̀, a propos du „live populaire" suédois Hilktrgardis och Tulundus (une variante de notre légende), entreprit avee plus de surcès, dans ses Svenstit Folkböcker, nne espèce de gronpement des diflérentes versions connmes ${ }^{3}$; mais il n'a pas examiné le rapport des gronpes entre enx. 11 ronsidere la rersion orientale (persane), qui est la plus riche en épisorles. comme lat plus anciemne et attribue par suite à notre conte me origine orientale". T'out opposée est l'opinion de Ŝr. Grunotrig, exprimée dans son grand onvage à juste titre célèbre, Dammarks yamle Folkeviser $(1553)^{5}$. Il rejette absolnment l'idée d'une origine orientale de la légende, parce quiil regarde le conte de la femme chaste convoitre par son beuu-fière comme intimement lié anx différentes versions dn cycle général de „la femme imnocente persécutée". dont la ballade danoise Racenguard oy Memeriny hi semble représenter le type le plns ancien. La version orientale que commaissait Grundtvig, celle des Mille et un Jours de Pétis de la Croix ", ne serait donc qu une simple imitation de quelque version occilentale ${ }^{7}$. Même la remarqne faite plus tari (en 1s60) par F. Liebrecit ${ }^{s}$ que notre conte se rencontre anssi dans los Mille et une

La version arabe, R. F. Burton, supplemental Nights to the Bonk of lle Thousund Nights and a Night 1586-1855], t. 1, pp. 94 [11]. et t. 11. p). 296-7. et R. Basset, Contes arabes [1843]. pp. 67-78: pour la version mügrure, A. Jaubert dans le Jomnal Asintique. t. X [1527]. 1). 150 7, et Davids, A Grammar of the Turkish language [14:32]. p1. $171-8$ ).

3) Lu fille dons le coffre, dans G. Meyer, Hbanesische Märchen, dans Archiv für Littcraturgesrhichle. t. XII (ISst), pp. 127-32 (No. 11: J)as Mädehen im Kasten). 1'. Lereh, Forschungen ïber die fiurden und die Iranischen Vordchaldäe, I (1457), pp. 33-9 (No. 6: Erzählung von I)acribeg. en kurde, avec tradurtion alle'mande;; et E. Prym et A. Socin, Liurdische Sammlungen. Erzählungin und Lieder in den Trialehten des Tür

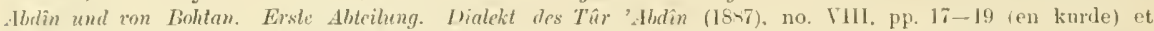
27-32 (en allemand: Jusif Buschari). A ce conte se rattathe encore partiellement un conte néo-aramérn,

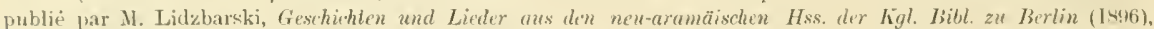

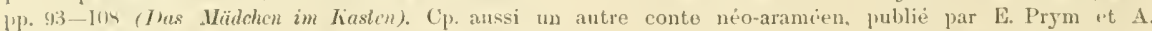

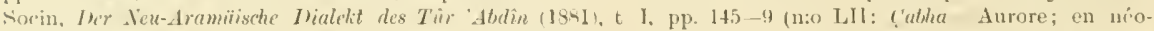
araméen), et t. II, PP. 2J1-16 (trad. allemande), où il y a mélange avee le conte connu du Pèré umourentr sle sa fille.

1 la désignation commune, conle de Criscentia. provenant du nom tonué à lhéroüne dans la version 1. lit haserchronik, nous semble trop spériale et, par lit, trop arbitraire.

P'p. $286-7$.

T. 1 (1845), pp. 264-74: v. aussi t. 11 (1844), pp. 6-7.

1. omer. rité. t. 1, p. 264

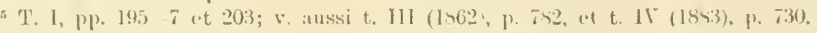

a Y. t. Y (éd. 172!) , pp. 241-953 (Histoire de lípsima).

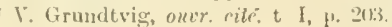

lahrb. i. rom. 1, angl. Lit.. 11, 131 
Nuits $^{1}$ ne changea en rien l'opinion de Grundtrig ${ }^{2}$. En comparason arec l'expose de Bäckström, celui de Grundtvig a le mérite de vouloir suive le développement historígue de la légende ${ }^{3}$.

'Trois ans avant l'apparition du premier volume du grand ouvrage de Grundtvig (en 1550$),$ F. H. VoN DER HAgex avait de son côté, à propos de sa publication de la version allemande de C'rescontia, discuté, dans ses Gesummlabenteuer', les vicissitules de notre légende. Son examen, qui se fonde principalement sur l'exposé de Grässe, est

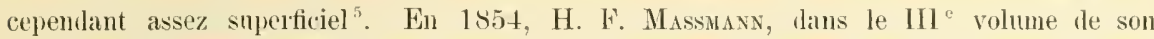
édition de la Kaiserchronik, fournit, à propos de lépisode Narcissus oder C'rescentiu de la vieille chronique, de nouveaux détails su les pérégrinations du conte". Il no reussit cependant pas a bien faire ressortir le rapport des différentes versions entre elles?

Les onviages que nons venons de mentionner contribuèrent snccessivement à élargir la connaissance des différentes rersions de notre conte, mas ils ne rénssirent pas à classer ces versions d'une manière systématique. Ce ne fut qu'en 1865 que Adotf Mussafı, dans un mémoire remarquable par sa clarté et sa précision, Über eine italienische metrische Durstellung der Crescentiusage", donna 111 classement méthodique les différentes versions. Son classement est fonté, non sur les divers degrés de développement du conte (jrobablement Mussitia jugea inutile de luasarder des conjectures incertaines), mais sur un point de départ tont conventionnel, qui est cependant, en même temps, en quelque rapport avec les différentes phases historiqnes du conte: le nombre des personues malades et gnéries par l'héroïne. Ainsi, dans le groupe I (disignation de Mussafia) il n'y a, en règle générale, qu'un seul malade: le beau-frère; dans le groupe II il

1 V. léd. de Habicht-Hagen-șchall (3:e èl., I834), t. X1, p1. 243-54 (nuit 497): Abenteuer eines Kadi's und seiner Frau.

2 V. Grundtvig, ouvr. cite, t. 111 (1562), p. $7 \times 2$.

a Il est surprenant de rencontrer, dans l'exposé si clair et méthodique de Grundtrig, une version qui n'appartient ancunement ì la légende en question, mais à la légende de „la méchante belle-mére": c'est le Mirurle du roi Thierry et de su femme Osanne. publiè par Monnerque et Fr. Michel dans leus Theître français an moyon-age (1539), pp. 551-605. (V. Grundtvig, owwr. cile, t. 1, p. 196).

+ I. $1, \mathrm{pp} . \mathrm{C}-\mathrm{ClV}$.

5 Lassertion erronée de von der Hagen ıue notre légende se retrouve sous forme de "livre populaire", dans le hollandais (Florentina de gutrowwe. v. ouvr. cité. p. CIV) provient évidemment d"un passage hátivement lu dans Grässe. Celui-ei dit expressément (v. lomvr. rite, p. 2\$i): "Wohl aber ist zu bemerken, dass durchaus ein anderes Buch, eine Art Familiengeschichte, ist das oft damit verwechselte Niederlandische Volksbuch: De Historie van Florentina de getrouue" ....... 906 et 910

- V. Massmann, Der keiser und der liunige buorh oder die sogenannte Kaisteleronit, t. 111, pp. $896-$

7 Ainsi la version d'Hild'garde (1). 9/0) est séparée des autres versions par nne analyse de deux versions étrangères au conte de la femme chaste convoitée jar son bcau-fière (Die unschuldige liönigin von Frunlireich et Die Königin sibiliu, p1.907- I0), et une version de notre conte, tirée des Gesta Romanorum alle mands, est placée à la suite du eonte de la fomme persécutée qui perd ses deux infints (v. pp. 913-6).

${ }^{8}$ V. sitzungsber. d. pluil.hist. Cl. der Krais. Akal. der Wiss., t. Ll, pp. 589-692 (Vienne, anuée 1565). Le mémoire a aussi paru en extrait (1866) 
y en a quatre, dont denx sont des persecuteurs de l'héroïne; dans le groupe III, ce ne sont que les deux criminels du groupe précédent qui sont malades; le groupe IV jrésente quatre criminels malades; enfin, le cinquième (et dernier) groupe ajoute aux malades du groupe précélent mu cinquieme, le mari'. Dans ce classement ne sont cependant pas comprises les versions orientales, dont Mussatia se contenta de dire qu'elles se rapprochent des grompes $I V$ et $V^{2}$. Q Quant à la version la plus ancienne et à l'origine du conte, Mussatia émit me nouvelle théoric. Selon lui, le conte serait venn de bonne heme de lorient dans les palys occilentaux sous une forme plus on moins simple et $y$ aurait pris les formes I-III; ensuite nue antre version orientale, celle-là encore conservée, aurait domné naissance aux groupes $I V-V^{3}$.

Depuis ee mémoire si consciencienx de Mussafia vante (1866), en guise d'appendice, un autre article sur le même sujet: Eine altspanische Prosudarstellung der Crescentiasage, notre conte n’a pas été, dans tonte son étendue, l'objet de recherrhes spéciales. De nouvelles rontributions d'une certaine importance à la liste des différentes versions du conte ont été domnées surtout par $H$.

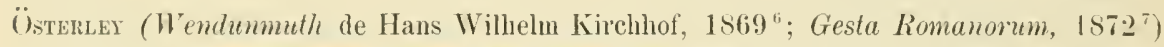
et W. A. Choustox 'Originuls and Analogues of some of Chaucer's C'anterbury T'ales, edited by F. J. Furnivall, Edmund Brock and W. A. Clouston, 1888) ${ }^{\text {s. }}$

1 Mussatia, ‘lans son mémoire, donne p. 678, note 1, un petit tableau des personnages malades des différentes versions de la légende. Il y règne cependant une rertaine confusion i la suite de quelque lapsus ealami; an lien de II il faut lire: III, au lieu de III: II, et au lieu de III $\theta$ : II d.

${ }^{2} \mathrm{~V}$. Mussafia, outr. cité, pp. 678-80. An lieu de: "Nit IlI und $V$ berührt sich zunächst die arabische Erzählung ${ }^{4}$ (p. 6i- ), il faut naturellement lire: „Mit IV und V* ete.; cp. p. 680: „Der innige Zusammenhang zwischen diesen orientalischen Versionen und den unter IV und V angeführten occidentalischen" - - -

${ }^{3}$ V. Mussafia, ouvr, cité, pp. 680-1: ,es ist daher sehw wahrscheinlich, dass die Sage schon in einfacherer Gestalt den Westen zugeführt worden sei, dort bald in gesehichtlicher, bald in legendarischer Form sich localisirt habe uud dass nur der französische Romun sammt seinen Ablegern (worunter auch die Versionen der Gesta Romanorum [- IV'] zu rechnen sind) von jener ausfülnlicheren orientalischen Version beeinflısst worden sei, welche in den arabischen Erzählungscyklus Aufnahme fand."

+ Lo mémoire fut reproduit en abrégé par M. Al. d'Ancona dans ses Sacre Rappresentazioni, t. III (1572), pp. 210-6 (en italien).

${ }_{5}^{5}$ V. Silzungsber. der kais. Akad. der Wiss., philos.hist Classe, t. LIII (Vienne, annė 1866), pp. 199-565. Ce mémoire a aussi para en extrait.

- T. V, p. 75 (II, 23: Von könig Carolo magno tin ware histori).

7 P. 747 (249: Hilllegarde). Dans la liste des versions donnée par M. Osterley il y a yunclques erreurs: 1) la version de fautier de Coinsi se trouve indiquée trois fois (Méon, 2, 1; - Gantier de Coinsi, de l'omperatrice etc.; - Méon, nouv. rec. 2, 1); 2) le renvoi "J. B. Dahl, Hildegardis, 1s32“ indique sans doute louvrage de J. Konrad Dahl, intitulé Die heilige Hildegardis, Abtissin in dem Kloster Rupertsbery bu Bingen (Mainz. 1 532 ); mais, comme le titre de louvage le dit dejjè, il y est question de sainte Hildegarde, contemporaine de saint Bernard, laquelle n'a jamais en rien à faire avec notre légende; 3) le renvoi ${ }_{n}$ Grässe, lit.

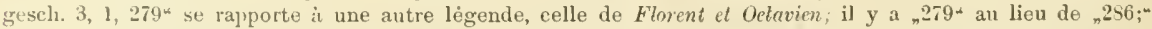
4) les versions de Straparola (Pincevoli nntli 1, 4) et de Basile (Pentamerone, no. 22) n'appartiennent pas à notre conte, mais au ronte du père amoureux de sa fille (cette dernière erreur se tronvo déjà dans l'édition (u Wendunmuth par 11 . Österley).

- Pp. 365-414 (No. 18. The Innocent Persceuled Wife. Asiatic and European versions of Chauecr's Man of Larr's Tale. Némoire publiè à part déjà en 1Ss6) et $5+1-3$ (Additional Notes). 
Nous avons dit ci-dessus ( $p .5)$ que Mussafia, le senl qui ait jusqu ici tâché de grouper systématiquement les différentes versions de notre conte, avait domné un classement tout conventionnel, basé essentiellement smr le nombre des persomages malitdes. Il est évilent (et nous sommes sûr que Mussafia aurait été le premier ì le reconnaître) qu'un tel classement ne peut être qu'un simple expédient. Ce qu'il faudrait, ce serait naturellement un classement oì l'on indiquerait d'abord la version la plus ancienne, la source même, ou, si la source est perdue, son dérivé le plus proche, pour snivre ensuite méthodiquement la marche de la légende à travers les pays et les îges. Un tel classement a naturellement le grand désavantage d’être presque toujours plus. ou moins lyypothétique; car il est très rare, dans ces sortes tle recherches, de pouroir i chaque pas établir un fait assuré. Dans beanconp de cas, jl ne pent s'agir gne d'une conjecture plus on moins bien londée. Si donc nous adoptons, pour le présent ouvrage: un tel classement rationnel, ce n'est pas que nous osions espéres d'aroir toujours trouvé la solution juste; nous le faisons, simplement parce que nous voulons avoir lis conscience nette davoir fait ce que nous avons pu pour arriver à un classenent vraiment satisfaisant des différentes versions tle notre conte.

\section{CHAPITRE II}

\section{Origine du conte}

Nous avons déjả mentionné, dans le chapitre précédent (pu. 4-(i), les trois opinions différentes qui ont, jusqu’à présent, été émises sur la mrovenance de notre conte: 1) origine orientale (Bäckström), -2) origine occidentale, plus spécialement germanique (Grundtvig), et 3) origine orientale à deux reprises (Mnssafia). Ajoutons que presque tous les savants qui, sans avoir entrepris le classement des différentes versions de notre conte, ont émis une opinion sur sa provenance, se sont montrés partisans de la thérie d'une origine orientale, c'est-à-dire indienne ${ }^{1}$. Pour dire tout de snite notre opinion, nous sommes aussi d'aris que le conte de la femme chaste convoitée par son beaufrère est né en Orient, probablement dans l'Inde, et que, contrairement à l'opinion de Mussafia, elle n'a pas, après sa première introduction en Europe, subi une influence orientale postérieure.

1. V. p. ex. Th. Benfey, dans Gött. gel. Anz. 1858, pp. 538 9; G. Paris, Hist. poét. de Charlemngne (1865), p. 396 ("L'histoire est d'origine orientale"); W. A. Clouston dans Orig. and Anal. etc. (1s8s), P. +14 ("I am disposed to consider the Innocent Persecuted Wife as of Hindú, if not of Buddhist, extraction"). 
Examinons labord la thorie de Grundtrig. Son principal, on pourait presque dire son unipue argument est que, comme notre conte est me simple branche du grand cycle de contes de la frmme innocente persecutie, dont il place lorigine dans le Nord, elle ne pent pas être 'n même temp̧s d'origine orientale. Grunttrig attache spécialement de limportanee au nom de Jrotrich, porté par les denx frères (le mari et le bean-frère) dans lancienne version allemande de Crescentia; car. selon hui. il y a là un indice de la parenté de cette version aver les légendes allemandes où figure mu Vietrich, rellet plus on moins direct du célèbre roi ostrogoth 'T'hodoric'. Liargument nous semble cependant bien faible. (Quand même le nom de Dietrich de la version Crescentia serait dî a l'influence d'un conte allemand sur le roi ostrogoth (ce qui nous paraît, d'ailleurs. assez problématiqne), il ne snivrait nullement de là que la version ('rescentia dût appartenil an grand cycle germanique de la femme innocente persécuté: un nom célèbre a très bien pu être donné par l'autem de la rersion Crescentia aux deux personnages yni, dans la tratition populaire, n'étaient probahlement comms que sous ces noms: l'emperem de Rome et son frère. Si, comme le fait remaryuer Grundtrig ${ }^{2}$, lihistoire des denx Dietrich et de la belle Crescentia est placée, dans la Kaiserchronik, au temps même où vivait le roi ostrogoth (avant Justinien), cela prouve tout an plus que l'anteur de la Kaiserchroniti a cru roir dans le mari de la chaste Crescentia son homonyme ostrogoth. Le fait que notre conte se retrouve dans des collections de contes orientales, telles que le 'Touti-Nameh de Nakhchabi, l'Al Faray Bairla Alsidda persan, les Mille ct une Nuits, etc. (v. ci-dessous chap). IIl), prouve déjì suffisamment, à notre avis, que l'origine du conte est à cherchel en Orient.

Quant à la théorie de Mussafia. selon laquelle la légende est bien de provenance orientale, mils qui, pour les versions occilentales, admet une influence orientale i deux époques différentes, elle n'est fondée smr aucun argmment spécial. Mussafia tronve simllencut très hasadenx (. änsserst bedenklich“) de conjectmer une élimintion sucessive d'épisodes originaux. ce quil fant éridemment faire, si lon regarde les versions orientales, avec leurs quatre criminels malades, comme représentant une forme plus archä̈que du conte que les rersions emropéennes I-III. Or, Mussatia ne parait pas avoir remarqué que tontes les versions .ocridentales" présentent un trait important qui ne se

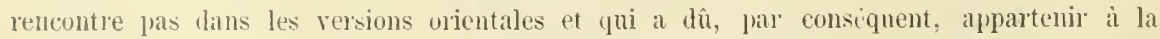
source commune de toutes les versions occidentiles, en opposition à leur source orientale:

T. Grundtrig, ouvr. cité, t. I, PP. 195 et 203. Grundtvig semble anssi voir un reflet de ce personnage historiqne dans le seigneur Thierri de la version de Florince de Rome (v. ci-dessous chap. V): cp. ce que dit Grundtvig, p. la6, sur Thierry, personnage du miracle lu roi Thierry et de sa femme Osanne P. P.

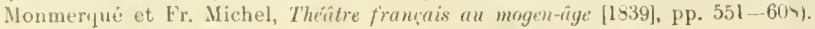

2 T. I, p. 19.5. 
le beau-frère, à la suite de ses premiers efforts pour sédurire l'heroün, est enfermé dims un lieu solitaire, d'où il ne sortira qu'au retour de son frère, trait qui n'a gucre pu être inventé à deux reprises. Nous nous royons donc antorisé à rejeter la théorie de Mussafia, quelque singulière que puisse parâtre, clans notre classement, l'élimination sncessive d'épisodes originan. Cette simplification de la fable primitive, telle qu'elle se présente dans les groupes I-III de la classification de Mussatia, nous l'expliquons comme due anx effets de la tradition orale. tandis que les grompes IT $-T^{T}$ nons semblent plutôt témoigner đ’une tradition littéraire.

\section{CHAPITRE III}

\section{Versions orientales}

Nons avons dit plus hant (1. 7) que nous croyons notre conte d'origine indienne, comme la plupart des contes répaulus en Orient. Malgré nos recherches, qui ont dû rependant se horner an déponillement des rerreils de contes indiens accessibles en langnes européennes, nons n'avons pu retronver cet original suppose. Il a pent-être fait partie dn famenx recueil de contes sanserit perdu dont le Sorkusaptuti (ou Les soixante-tix conles d'un Perroutuet) actuel ${ }^{1}$ est le descendaut appaurri. Nons pourons le supposer arec me certaine raisemblance, puisyne nous retromons notre conte de la femme chaste convoitie par son beau-frère daus un recneil de contes persan dn premier. tiers du XIV" siècle, apuelé Touli-Nameh (Le livre du Perroquet) et ayant pon antem. mu certain Zijyâi-ed-dîn-Nakhchabî. Or, ce Tonti-Nameh n'est que le remanienıent d'un Touti-Nameh antérieur perłu, qui était me traduction plus on moins altérée (probab]ement dn XII" siécle) du Sonkasaptati indien primitif ${ }^{2}$.

La rersion indieme, source supposée de tontes les rersions du conte de la femme chaste convoitee par son beau-frère. étant intronvahle, il est impossible de dire quelle a été exactement la forme primitive de notre conte. Une comparaison des différentes versions orientales entre elles nous conduit cependant à une source commune (indiemne) qui n*a pas dî être très éloignée du vrai original. Partant du principe, d'ailStuttgart 1599 )

II en existe une bonne traduction allemande par Rich. Schmidt (Dic Sukasaplati - texlus ornatior.

${ }^{2}$ V., pour liceuvre de Nakhchabi, Yétude détaillée de W. Pertsch dins la Zeilschr. der Drutschen morg. Ges., XXI (1867), 505-5! (cp. XXII, 568). Nakhehabi composa son Touti-Nameh, l'après ce qu'il en dit lui-mème à la fin de son wuvre, lan 730 de l'hégire, cest-ì-dire en 1330) après J.C. (cp. Pertsch, art. cité, p. 5166). M. II. A. Clouston (Orig. and Anol. etc., p. 310) avance, sans preuves ì l'appui, gue le ToutiNumek de Nakhchabi a été composé nenviron $1306^{*}$; cp. R. F. Burton, Supplem. Nights to lhe Enok of the Thousand Nights and a Night (Benares 1856 -1885), t. 1I, p. 297. 
lems tont théorique, que cette version primitive a été simple et logique dans ses détails. uous nous la représentons à peu près sous la forme suivante ${ }^{1}$ :

I'n homme (1), voulant entreprondre un voyage, eanfia sa femme (2) ì la garde de son frère (3). Celui-ci devint amoureur de sa belle-saur, et, eomme elle repaussait ses propasilians déshonnítes, il laceusa d'adultère devant le juge du pays. Acrusce par de faux témoins soulayis par son beau-frère, la femme ful enndamnéc à titre lapiéé. Laissíe ì moilté morte sur le lieu du suppliee, elle fut recucillie par un passant misérieordieux (1), qui la conduisit duss sa maison et lui confia san fils enfant ì gurder (a). -- Un esclave de la maison (5) tomba amoureur de l'héroine; rebuté par elle, il rísolut de se venger. I'ne nuit, il s"inlroduisit dans la chambre oit dormait lenfant de son maitre it le tua, puis il eutra dans la chambre de sa gardienne, lacha ses habits de sang et cacha près à elle If couteau ensanglante. Le lendemain, quand le meurtre eut c'té découvert, leselave altira les soupesons sur l'étrangère². Lhâte et sa femme ne purent ecpendant pas se convainere pleinement de sa eulpabilité: ils se cantenterent de la renvoyer, th l'hôte compatissant lui donna mîme une somme diargent pour son voyage (b). - Avee eet argent elle rurheta un jeune homme quion allait pendre paur dettes (ii). Par reconnaissanec, le jeune homme l'aecumpagna, mais devint amoureux d'elle. Repousse, il la vendit comme esclave au capitaine d'un navire (c). - Celui-ci (7) lamena ì bord de son vaisseau et voulut user de ses droits de maître envers sa uouvelle eseluve. Mais, sur le's prières de celle-ei, une violente tempîte séleva qui brisa le navire. Lihroüne el le capitaine furent sauvés, chacun de son côté (d). - La femme traùa un refuge dans un couvent, où, grốce à sa saintelé, elle guérit toules sorles dé maladies (c). - Pendant ce temps, ses quatre perséeuteurs avaient été frappés de maladics diverses, et son mari. revenu, avait appris par son frère sa enupabie conduite. Comme le renom de la sainte femme qui guérissait tous les maux étrit arrivé jusquaux oreilles du mari, il se mit en route avec son frère malade pour chercher auprès delle la guérison de eelui-ci. Ein chemin, sé joignirent à eux surecssivement le bon hôte avec son esclave malade, le jeune homme rarheté et le capilaine (f). - Arrivés à destination, ils furent admis auprès de la femme, qui. couverle d'un voile, leur ordonna the raconter fidèlement ce quils avaient sur leurs conscienees. Ils racontèrent alors l'un après lautre (le mari parlant le premier) ee qui se rappartait ì leurs relations avee la fomme. Celle-ei se fit connẫtre, pardonna aux eriminels et les guérit, après quoi clle retourna avee son mari dans leur puys, où ils vieurent heureux $(g)$.

${ }^{1}$ Pour simplifier les renvois à cette version primitive, nous y désignons les personnages principaux par des chiffres $(1-7)$ et les différents épisodes du récit par des lettres minuscules (a-g).

${ }^{2}$ Le trait quiun meurtre est imputé ì une personne innocente par l'assassin lui-même se retroure souvent dans les contes populaires. Mentionnons iei les contes suivants:

un conte néo-aramén, où un sénéchal tue l'enfant de son maître et accuse lu meurtre la femme du maître (v. E. Prym et A. Socin, Der Neu-Arame̋ische Thalcht des Tû̀ 'Aldîn [18S1], t. I, pp. 145--9 et t. II, [P. 211-6 ['ubha]):

un conte serbe, oi c'est la méchante belle-sceur gui tue son propre enfant avee le conteau de l'heroine et cache celui-ci sous loreiller de la femme innocente (v. H. F. Massmann, Der keiser und der kunige bunch ader die sogenannte Kaiserelironik, t. III [1854]. pp. 916-7);

un conte slovaque, oủ c'est lo pére de la femme qui tue ses propres petits-fils et place le conteau ensanglanté sous l'oreiller de sa fillo (v. Zeilschr. für deulsche Mylholagie und Sittenkundt, t. IS [1859], pp. $2: 1-8$ (1) Her thelf]):

un conte sicilicn, oú c'est un religieux, amoureux de la femme, qui tue les enfants de celle-ci et place le coutean dans sa poche (v. L. Gonzenbach, Sicilianisehe Wïrehen, t. I [1870]. pp. 153-7 /[on dem hinde der Hutter (rottes]).

un conte de l'Armagnac, où e'est la marâtre yui tue sa propre fille et accuse du menrtre la fille de la premiere femme de son mari (v. J.- Fr. Bladé, Contrs at proverbes populaires recueillis en Armagnae [1s67], p]. 55 6 [La dimayseleto]).

Dans les Pizecvoli Natti de Straparola, l'histoire du "père amoureux de sa fille" (I, 4) raconte comment lo pire se renge de sa fille, devenuo reine d'Angleterre, en tuant les deax enfants de celle-ci avec le coutoau même de la reine, qu'il remot ensuite dans sa gaine. Comme la scéne manque dans la source des l'úcevoli Xatti, ll Pecorone de Sier (iovanni Fiorentino, ouvrage composè en 1378 (Giorn. X, no. 1), il est possible

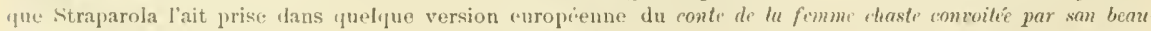
frère. Pour des contes apparentés à celui te Straparola. v. encore G. Rua, Intorno alle .Piarevoli notli" drllo straparola (159(y), pp. 4x-9. 
Les versions orientales qui nous ont été conservées, et dont aucune n’a gardé intacte la version que nous regardons comme la primitive, penvent être divisées en trois branclies principales: celle dı Touti-Nameh, celle des Mille et une Nuits et celle des Mille et un Jours.

Si nons examinons d'abord la branche du Touti-Nameh, il fant en premier lien mentionner la version de Nakhchahi, dont il a déji été question ci-dessus (p. 9). W. Pertsch en a domé une analyse létaillée daus la Zeitschrift der Deutschen morgenländischen Gesellschaft, tome XXT (1867), ${ }^{1}$ sous le titre: Geschichte von der Chôrschîd und dem 'Utürid (33 ${ }^{\mathrm{e}}$ nuit). Voici les traits principaux par lesquels cette version diffère de la version primitive:

Personnages: (1) 'Utârid, écrivain publịc; (2) Chôrschîd; (3) Kaivân; (t) le beall-père de Chôrsehîd; (5) Latîf, second beau-frère de Chôrschíd; ${ }^{2}$ (6) Sicharîf; (7) un marchand, passager sur lo navire. Episodes: (a) c'est devant l'émir que Kaivân aecuse sa belle-şeur; les faux témoins manquent; (b) l'enfant, frère de Latîf, luabite la même chambre que Chôrschîl; il n'est pas question du couteau ensanglanté; Chôrschîd ne reęoit pas d'argent pour son voyage ${ }^{3}$ (c) Scharîf est battu par des hommes, ḋ la suite d'une rixe; il s'imagine que Chôrschíd l'a racheté, paree qu'elle est tombée amoureuse de lui; (d) effrayé par l'orage, le marehand promet de laisser Chôrschîd en paix; l'orage cesse, et le narice aborde la terre; (f) lo bean-pére n'est pas du nombre des personnes qui vont cousnlter Chôrschíd, ni naturellement le marehand; (g) Chôrschî́l amène la confessiou des trois criminels en leur racontant une histoire de son invention oú leurs méfaits sont décrits.

Le Touti-Nameh de Naklıchabi parait avoir joni d'une très grande répntation, puisqu'il a été plusieurs fois remanié et trałuit en différentes langues orientales. ${ }^{5}$ Cependant, autant que nons arons pu le constater, notre conte ne se trouve pas dans ces remaniements et traductions. ${ }^{6}$ Mais il y a un remunicment turc du Touti-Nameh

' Pp. 536-8. Le Touli-Nameh de Nakhchabi n'est pas encore publié dans la langue originale. Ine traduction française manuserite, probablement du milien dn XVIIJ:e siécle, se trouve a la Bibl. Royale de Munich (Mss, orient. 174-176,3 volumes in-folio; le texte persan à gauche, la traduction française à droite). Notre conte s'y lit t. II, pp. 135-49 („Conte \& soirée 32:e de la rare beauté de Courchide, et des malheurs qui lui survinrent par l'effet de sa beaute $\left.{ }^{\star}\right)$. Cp. J. Aumer, Hie Pers. Hss, d. K. Hof-und Staalsbibliolhek in Wiünchen (1866), p. 54. Pertsch connaissait l'existence de cette traluction française, mais ne l'avait pas lue (v. Pertsch, art. cilé, p. 510: no. 4).

2C'est une altération évidente du récit primitif que les rôles de l'hôte misérieordienx et de son esclave soiont joués par des parents de la femme.

${ }^{3}$ L'argent pour le voyage est nécessairement un trait primitif, puisque sans rela on ne comprend pas aver quoi l'héroüne aurait pu racheter le jeune homme. Il est invraisemblable qu'elle l'eut fait avec n'or et les bijoux qu'elle portait sur elle", comme le dit la version de Nakhchabi, car comment l'aurait-ou lapidée avec tout eela?

* Ce trait, que l'hérö̈ne provorgue les confessions des coupables en racontant, sous une forme plus ou moins déguisée, sa propre histoire, se retrouve dans un autre conte d'origine orientale, que nous avons sigualé ci-dessus p. 3, note 1 (sous 1), dans des versions arabe, néo araméenne, grecque, russe et finnoise.

5 V. Pertsch, art. cité, pp. 506 -s, et t. XXII (1868) de la même revue, p. 56s; cp. R. Sichmidt, Fier Erzühlungen aus der (Cukasaptati (1890), 1.9. 9.

- Nous arons consulté la traduction allemande du Touti-Namel de Mohammed Qúdirî, par C. J. L. Iken (Touli Vameh. Eine Simmlung persischer Mïhrchen won Nechschebi. Stuttgardt 1522), ainsi que la traduction anglaise de la rédaction hindoustanique du même ouvage par J. Gilehrist (Totu Fulunce; a translation of the popular Persian tales, entitled Tuthi Nameh. 2:0 éd., Calcutta 1810). 
primitif, probablement du commencement du $X T^{\text {e }}$ siccle ${ }^{1}$, qui donne notre conte sons ce titıe: Histoire de Merhûma. De cette rédaction turque nons connaissons différentes éditions ${ }^{2}$ : ainsi que deux traductions en langue allemande, l'une par G. Rosen ${ }^{3}$, lautre lar M. Wickerlauser ". En outre, on trouve notre conte, probablement d'aprés la version de Rosen, sous le titre La Tertu dune femme dins l'unve de Xavier Marmier ${ }^{5}$. Ial rersion tmque du Touli-Nameh différe de la version primitive par les traits snivants ${ }^{6}$ :

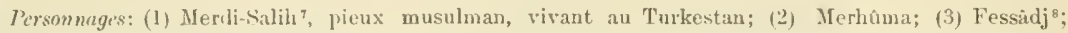
(4) un Bélouin". Fípodes: (a) le Bédonin, frappé de la leauté de Mlerhûma, propose de l'épouser, mais abandonne son projot, "quand il apprend qu'elle est marice ${ }^{10}$; (b) aprés avoir déconvert le meurtre ${ }^{11}$, le Bédouin maltraite d'aborł Merlúma, mais se laisse ensuite convaincre de son innoeence; il la renvoie, de peur que sa femme ne lui fasse du mal; (d) non seulement le capitaịe, mais tout l'èuipage veut porter atteinte à la vertu do Merhania; tout le monde, excepté Merhôma, est tué par un coup de foudre; elle arrive seule sur le navire it un port lle mer ${ }^{12}$; (e) par précaution, Merhúma s'luabille en homme ${ }^{13}$ et va raconter ses aventures au prince de la ville; celui-ci, ayant reçu de Nerhuma le navire aree sa cargaison, lui fait bâtir un couvent; $(g)$ cest devant le Diran, convoqué par le prince sur les prières de Mlerhuma, que les trois criminels racontent leurs méfaits'.

1 V. G. Rosen, Tuti-Nameh, I (1859). p. XlV; e]). Th. Benfey dans Götl. gel. Anz., année 1858, 1. 532.

2 Eil. de Bonlac, 1434 [1253], dont lauteur aurait ité un certain Sary Abdoullali-effendi (r. Bianchi dans le Journ. Asial., IV:e série, tome If (1843), p. 45: no. 135); ed. de Constantinople, 1840 [1256] (v. IIamnor dans Jahrb. der Lil., t. X('VI [1541], ]. 106, note: no. 160). Nous ne saurions dire si l'édition de Kazau de 1\$51 (v. Zenker, Bibl. orient., t. II [1861], p. 51: no. 678) contient la même rédaction.

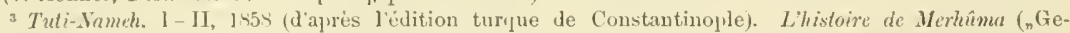
sclichte der Merhuna ${ }^{4}$ s'y tronve t. I, pp. 89-108.

+ Jie Papagcimärchen, 1458 (d'après l'élition turque de Boulac). Die keusche Merhuma se lit pp. $50-6$ (7:e nuit). Cotto traduction diffère seulement pour quelynes détails de celle de Rosen. M. Landau (Thie

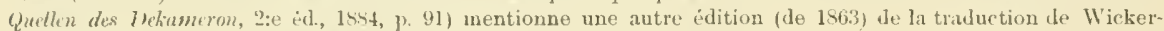
hanser, portant lo titre: The dreissig Närhte.

"Contes populaires de différenls pays. Denxième série (1898), pl. 165-77.

- Nons nous sommes servi de la traduction de Rosen.

? Dans la version de Wickerhauser: Salil, dans celle do Marmier: saliah.

- Alujelé Ferrudi par Narmier.

- Un Arabe, d'atjés Wickerhauser.

10 W'apres la version de Wickerlanser, la proposition n'a liou qu'au bont de quelques jours, dans la demeure de lihrabe.

11 fiaceusation directe par l'assassin, qui manque ici, a nécessairement dü se trouver dans la version primitive, puistgu elle se retronve encor* dans la plupart des versions oecidentales gui doment l'ópisode en Iluestion.

12 Cet ipisorle, M. de Gubernatis (Zoological Bythology [18:2], t. I, p. 121) le regarde comme le reflet d'un mythe solaire: $\rightarrow$ it is the aurora coming out of the gloomy ocean of night, and the monsters who persecute her are burnul to ashes by tho thunderbolts and the sun's rays." De mêmo, M. de Gubernatis dit à propos de la litpiclation de Merhoma couvr. rité, t. I, 1. 315): ,the aurora lost in the mountain of stone." Avons-nous besoin de dire oxpressément que nous ne pouvous admettre ce rapprochement fantastique de notre conte tout humain avec les vieux mythes solaires?

${ }^{13}$ Ce trait, assez superflı ici, mais qui se rencontre tres souvent dans les contes populaires orientaux (v. V. Chauvin, Bibliographic des ouvrages arabes, t. V [19011, p]. 96, note 1, et 295 [Add.]). ne se trouve pas dans l'idition de Wickerhauser, et a aussi été omis par Marmier.

1t Wappès la version de Wickerhauser, l'Arabe n'a pas accompagné son esclave, et la confession a lien tout de suite, diıns la cellule méme de Nerhúma. 
Comme on a pu le voir par les analyses précédentes, les denx rersions du ToutiNameh, quoique différant sensiblement entre elles, ont quelques traits en commun par lesquels elles diffèrent de la version primitive et forment nn groupe à part. ${ }^{1}$ C'est avant tont le fait que le capitaine (marchand) ne figure pas parmi les malales, quoique la logique du récit demande nécessairement que les personnes qui ont en directement à faire avec l'héroïne se retronvent toutes à la scène finale. Ensuite, il fant observer que l'héroïne ne fait pas nanfrage. Enfin, ce ne sont que les criminels qui racontent comment ils se sont comportés envers la femme. Voilà les traits caractéristiques de la branche du Touti-Nameh.

Passons ì la seconde branche des versions orientales, celle des Mille et une Nuits.

Dans les différentes rédactions de cette célèbre collection arabe de contes, qui, dans sa forme première, remonte an moins an $\mathrm{XIV}^{\text {e }}$ siècle $^{2}$, on trouve trois versions différentes du conte de la femme chaste convoitíe par son beau-frère. Celle qui, par la conservation de lépisode (d), est le plus voisine de la version primitive, se tronve dans le ms. Wortley Montague ${ }^{3}$, conservé actuellement à la Bibliothèque Bodléienne d'Oxford. Le conte y porte ce titre: Le Cadi de Bagdad, su vertueuse femme et son méchant frère. Cette version fut publiée en anglais, en 1811, jar Jonathan Scott daus ses Arabian Nights Entertaimments sous le titre: Adventures of the Canzee, his Wif'e etc ${ }^{5}$. Depais lors, elle a encore été reproduite en anglais ${ }^{6}$ et traduite, d'après la version de Scott, en d'autres langues ${ }^{7}$. Cette version, que nous appellerons la version Montague, diffère de la rersion prinitive par les traits snivants ${ }^{\text {: }}$

1 Que la version turque ne puisse pas dériver de celle de Nakhchabi, c'est ce 'Iui ressort, non seulement des analyses précédentes, mais aussi dine comparaison gẻnérale dn contenu des deux Touti-Tamch (v. Pertsch, art. cité, pl. 513-51).

${ }^{2}$ V. R. F. Burton, The Book of the Thousand Vights and a Night (London 1s94), t. V111, p. 79: „a study of the vie intime in Al-Islam and of the manners and customs of the people proves that the body of the work, as it now stands, mnst have been written before A. D. 1400,"

3 Tome VII, p. 77 et suiv.

- Daprès la table des matières de ce ms., donnée par J. Scott dans The Oriental Collections de W. Ouseley, t. II (1795), pp. 25-35.

5 T. VI, pp. 396-40S (nuits 900-911).

- Ainsi, par M. W. A. Clouston dans les originals and Analogues of some of Chaner's Canterbury Tales (1885), pp. 379-85. Cetto traduction diftëre un peu de celle de Scott.

7 En frençuis, elle se trouve dans les éditions des Mille et une Nuits d'Éd. Giautier, t. II (1823), pp. 406-18 (497:0 nuit: Aventures d'un Cadi et de sa femme), et do N1. Destains (1825), t. V1, pp. $300-13$ (Aventures du Cadi et de sa femme); en allemand, dans lédition de Habicht-Hagen-Schall (1825). t. XI, pp. 287-99; 5:e éd. (1840), t. XI, p1. 197-206 (nuit 497: Abenteuer eines Kadi's und seiner Frane.

${ }^{3}$ Nons nous sommes serri de la traluction anglaise donnée par M. Clouston dans les Urig. and. Anal. etc. (v, ci-dessus note 6 ). 
P'rsmmagis: (1) un radi, vivant ì Baydad; (t) un íleveur de chaneanx; (5) un chamelier. Episodes: (a) Ja femme est condamníe ì être fustigce et chassić; (b) le chamelier tue par migarde lenfant qui dormait is cóté de la femme, en voulant tuer celle-ci, aprés quoi il s'enfuit; la femme n'est pas soupconnée, elle continue tout simplement sa route; il n'est pas question d'argent de voyage; (e) c'est un sultan qui lonne it li femme une demeure près de son palais; elle ne guérit pas de malades, mais aequiert une grande réputation de sainteté par l'efficarité de ses prières en génèral; (f) le carli n'aceompagne pas son frère, qui a quittú la maison arant lui, mais ils se rejoignent en route sans se reconnaitre; ancun les voyageurs n'est malade: ils se rendent chez la femuc pour chereher absolution de leurs pécliés; (g) un homme de confiance du sultan assiste, caché, a l'entrevue, ot le sultan veut d'abord punir les quatre coupables; le cadi et sa femme restent aupres du sultan.

Une antre version des Mille et une Nuils, unui se trouve dams les éditions arabes de Boulac (1535-1836), de Calcutta (1839-1842), de Bombay (1297 l.), du Caire (1302 h.) et de Beyrouth $(1589-1890)^{1}$, porte le titre: Le c'adi juif el sa pieuse femme. Elle a été publice plusieurs fois en langues europermes, la première fois en 1823 par A. E. Zinserling en allemand, d'après une traduction francaise manuscrite, anjourd'hni perdue, faite par l'orientaliste J. ron Hammer ${ }^{2}$. Cette version, que nous appellerons la version Boulac, diffère par les traits suivants de la version primitive ${ }^{3}$ :

- Personnages: (1) un cadi juif; (4) un paysan; (5) un voleur; (6) un malfaiteur ${ }^{4}$; (i) manqque. Lipisodes: (b) - la version Montague ${ }^{3}$; la paysanne bat la femme et veut la tuer $^{8}$; le paysan ne lui donne pas dargent ${ }^{7}$; (c-e) on est en train de crucifier le malfaiteur; il n'est pas déloyal envers sa bienfaitrice; c'est lni, au contraire, qui lui bâtit une cellule, oủ il la sert fidèlement; l'épisode (d) manque donc entièrement; (f) les unalades sont le beau-frère, le voleur et la paysanne; ils ne se rencontrent qu'à la porte de la saiute femme, la paysanne accompagnée de son mari; $(\mathrm{g})$ seulement les criminels se confessent; tons restent auprès de la femme du cadi à servir Dien ${ }^{8}$.

\& V. la liste des contes des deux éditious nommées eu premier lieu dans J. Payne, Tales from the Arabic, t. III (1884), pp. 249-5!) (no. 118, nuits 465-466: The ,Jewish Cadi and his Pious Hife). Pour les élitions de Bombay, du Caire et de Beyrouth, v. la liste dressée par M. R. Basset dans la Rerue des tradilions prpulaire's, t. XI (1996), p]. 148-n7 (nuits 465-466: La fimme aceuse injustement [pp. 169-9]).

${ }^{2}$ Cette version se trouve daus les éditions suivantes des Mille ct une Nuits: 1) en allemanl: A. E. Zinserling, Der Tausend und Einen Vacht noth wiht äbersctzte Mährchen. Erzählungen und Anekdoten (1823-1524),

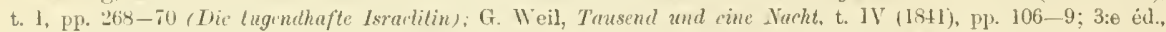
3:e imjr. (1589), t. 1V, pj). 85-7 (Die tugendhafte Fran eines israelitiscten Richlers); M. Henning, Tausend und eine Waeht (1895-1899), t. 1X, pp. 14 et suiv. (Der israelitische Fadi und scin frommes Weib): 2) en fransais:

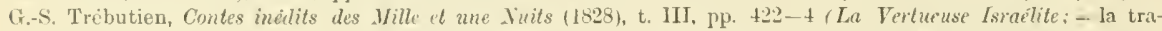
ductiou allemande de Zinserling); 3) en anglais: J. Payne, The Book of the Thousand Nights and One light, t. V (1N+3), 11). 9-13 (The Jewish Cati and his Pious Wife); R. F. Burton, I plain and literal lranslation of the Arabian Nights Entertainments, now cntituled. The Book of the Thousand Nights and a Night (Benares 1855 ), $t$. V. 11). 256-9 (The Jerish Kazi and his pious Hifc. version rejroduite par M. W. A. Clouston dans Originals and Analogues of some of Chaueer's C'antertury T'ates [1884], pp. 368-71); J. H. Mc Cartly, Laly Burton's Edition of her Ilusband's srabian Nights, t. 111 (1886), pp. 335-S (= la précédente version): R. F. Burton et L. O. Smithers, The Bnok of the Thousand Nights aul. a Night (London 1894), t. 1V, Pp. $206-9$ (= l'édition de 1885 ).

3 Nous nous sommes servi de la traduction anglaise de Burton.

* Un débiteur, d'aprés lés traductions de Zinserling et de Trébutien.

- Dans les versions de Zinserling et de Trúbutien, lassassin, dont la profession n'est pas indiquéc, tue l'enfant, parce que le petit commence à rrier.

- Si lon considere lattitude hostilo de la fermme du Bédouin dans la version turque du Touti-Nameh (v. ei-dessus p. 12), on est tenté d'admettre que ee trait est primitif.

7 Dans la rédaction Zinserling-Trébutien le paysan donne a la femme une somme d'argent.

${ }^{3}$ La fin du récit est abrégée et un peu différente dans la version Zinserling-Trébutien. 
Par le fait que, lans les versions Montagne et Bonlac. l'aswissin tne l'enfant par mégarde (ou, du moins, sans préméditation) ces deux versions paraissent ctre assez étroitement apparentées entre elles, malgré tontes leurs divergences.

Par ce même trait, me version juive, contenue dans le célihre Mause-Buch, composé frobablement dans le desuier tiers dn XVI" siècle par nu duif de l'Allemagne méridionale d'après différentes somres ${ }^{1}$, semble remonter à la même sonree qne ces denx versions arabes. Comme la version Boulac, la version juive raconte, en opnosition avec la version Montagne: 1) que la femme est lapictée, ef 2) que les criminels sont malades. D'antre part, la version juive doune (sons une forme trés altérée) l'épisode du voyage en mer, ce qui montre que la version Boulac ne peut pas être sa source. Voici les principatx traits par lesquels la version du Muase-Buch, d'après une élition, non datée, de Wilmersdorf (Bavière), chap. 203, diffère de la version prinitive ${ }^{2}$ :

Personnages: (6) manifue; (7) des pirates. Épisodes: (b) l'esclave reut tuer la femme, mais le tils de l'hôte (joune homme, à yui elle donne des leçons) se jette contre l'assassin et est tué, aprés yuoi la femme s'onfuit; l'épisode (c) manque; (d) la f('mme est prise par les pirates, qui, effrayés par un orage, tirent au sort pour savoir qui sera jeté à la mer; le sort tombe sur la pauvre femme, mais les pirates se contentent de la conduire à terre; (e) la femme se coustruit une cabane et apprend ì guérir la lèjre; (f) les malades qui viennent la voir (avec le mari) sont les (deux) faux témoins; (g) les lépreux ne sont pas guéris, vu la gravité de leur crime ${ }^{3}$.

Il est natmrellement difficile de dire qnel est le rapport exact entre la version Montague, la version Bonlac et la version juive. Par le fait yue, dans les denx dernières, il s'agit de la construction d'une dememre pour la femme, on pourrait stpposer qu'elles forment un groupe contre lit version Montague.

Nous arrivons maintenant ì la troisième version des Mille et une Nuits. Elle a été publiée en arabe, d'après un ms. tmnisien, dans l'édition dite de Breslan (18251.843) par M. Habicht et H. I, Fleischer, et porte ce titre: La pieuse Femme accusée de libertinage ${ }^{4}$. Elle a été deux fois traduite en anglais ${ }^{5}$. Cette version, que nous

1 Sur le Haase-Buch v. l'artiele de M. Steinschneider, Jülisch-deulsche Litteratur und Jüdisch-Deutsch dans Serapeum, t. XXVII (1จ66), pp. 1-12; cp. aussi t. XXV (1864), pl. 67-72. Différentes éditions sont citées par J. Chr. Wolf, Bibliothece Hebraca, t. II (1721), p. 1360 (no. 395), et M. Steinschneider dans sierapeum, t. IX (1848), p. 379 (no. 156).

${ }^{2}$ Nous nous sommes servi d'une transcription en allemand, faite penr notre compte par M. Heinrich Glaser, de Munich. Nous en domnons une traduction française dans notre Appendice (A).

${ }^{3}$ Dans le résumé que donne de la dernière jartie de cette version M. Max Grünbaum, Jüdischleutsche Chrestomuthic (1552), pp. 430-1, d'après l'édition de Rörlelheim (de l'annèe 1753) et celle 'lue nous avons employée, il raconte "que le beau-frère devient aussi lépreux et va arec les deux faux témoins cliez la femme persúcutée. Nous ne saurions dire si M. Grünbaum s'est trompé, ou bien si c'est vraiment li la scrsion de l'édition de Rödelheim. La logique du l'écit demande évidemment ¡que l'instigateur du crime, le beau-frère, soit également puni, mais, d'autre part, cette histeire veut spécialement prouver comment sont punis ceux qui portent faux témoignage (v. l'Appendice, A, la fin).

- V. la liste des contes do ce ms. daus J. Payue, Tules from the Arabic, t. IlI (1884), pp. 263-it (no. $110 \mathrm{r}$, nuits 907 et suiv.: The Pious Thoman acrused of Lewdness).

5 V. J. Payne, Tales from the Arabic. t. II (158t), pp. 5-16 (Story of the Pinus Homan accused of Lewdness), R. F. Burton, Supplemental Nights to the Book of the Thousand Nights and a Night (Benares $1856-$ 
appellerons la version Broslau. presente par raphort il la version primitive les divergences que voici':

Personnages: (1) un homme de Nichapour; (4) un cheik; (5) le tils du cheik; (i) manque. Épisodts: (a) lit frome est lajidée par des gens nui eroint l'accusation du beau-frére; (b) le fils du clecik, pour se veiger, suburne un autre jeune lomme, qui s'introduit dins la demeure du cheik, vole quelyues objets, se laisse arreter et arense la fenme dritre sa complice (done pas cle menrtre); (e) le dibiteur doit êtro fustigé; il déuoure sa bienfuitrice comme uspion au sultan du pays, mais elle a le temps de senfuir; (d) manruv; (o) la femme, habilke en homme? arrive daus une grande ville, ou elle actpuiert l'amitie de la fille du roi; le roi mort, les gens de la ville veulent tuer la princesse et son prétendu amant; la princesse est, en effet, massacre, mais, yumil la femme persicute lait comnatre son soxe, la haine se change en repentir, et on la prorlame reine; (f) le complice du fils du cheik est parmi les malades; (g) seulement les criminels racontent leur histoire; le mari est fait roi do lit ville.

C'omme l'épisode (b) est fortement altéré, il est llificile de savoir si la rersion Breslan duit être grompée avee les denx autres versions arabes, on hien si celles-ri forment grompe contre, elle. A l'appui de cette dernière alternative on ponrrait citer le fait que, dans la version Breslan, le cheik dome à la femme une somme d'argent pour ses frais de royage, trait surement primitif" mais qui manque dans les versions Montague et Boulac ', ainsi que dans la version du Maase-Buch. (Yuant à l'épisole (e), il parait être emprunté à quelque autre conte, où une femme, après s'être fait passer pour homme, est arrivée à tle grands honneurs ${ }^{5}$.

On remarque un rapprort assez étroit entre la version Breslau et une rersion tuture très comompne, domée par M. Wr. Radloff dans ses Proben der Vollestitterutur der 'Türkischen Stämm Sü̈l-Sibiriens sous le titre: Das Weib als Fürst ${ }^{6}$. La comparaison avec la version primitive dome le résultat suivant:

lersomnagis: (4-5) manquent; (6) un homme ivre. F́pisoles: (a) la femme est condamnée à être pentuo; elle réussit à s'échapper, en donnant de l'argent aux bourreaux; (b) manque; (c) simple rencontre ave l'homme irre; (e) la fenme est recueillio par un homme charitable; le vieux souverain de la villo veut abdiyner "t, pour trouver son successeur, il se sert d'un oisean magique qui doit se placer sur la tête

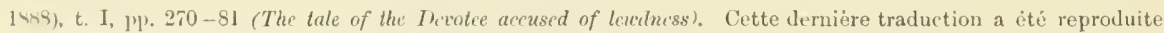

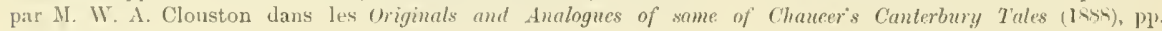
$371-8$, et dans la nouvelle édition par MI. L. C. Swithers (Loudres 1s94) do louvrage de Burton, t. IX, 1). $177-84$.

1 Nous nous sommes servi do la traduction de Burton.

- Cp. ci-dessus p. 12, note 13 .

${ }^{3}$ Cp. p. 11, note 3. Déjì le manque de ce trait daus la rersion Boulac nous empeche de regarder, avec Burton (siupl. Nighls ete., 1, 270, note 1; èd. de 184, IX, 177, note 1), la version Breslau comme um simple "rechaufici de la version Boulac. En outre, la trahison du débiteur racheté manjue dans eette derniere version.

+ Il se rencontre cependant daus la version Zinserling-Trèbutien (v. ci-dessus p. 14, note 7 ), qui présente certaines altirations secondaires do la pure version Bonlac.

- Voir p. ex. le conte arabe des lmanls de Syrie (1. Gauttier, Les Mille et une Tuits, VI [18:3], $349-60$ ) et le conte apparenté de la Fille dans le coffre (r. ci-dessus p. 3, note 1). Cp. p. 12, note 13. ville de Tara).

- V. t. IV (1872), pp. 141-5 (reeneillie parmi les Tatares de Chodseha Aul, au nord de la 
de la personne élue'; c'est ainsi que la fe'mme devient souveraine de la ville; (gr) les malades sont guéris au moyen d'une boisson; le mari devient roi ì côté de sa femme.

C'est à canse de la hante position que parvient à obtenir la femue aussi bien dans la version Breslan que dans la version tatare que nous regardons ces deux versions comme apparentées entre elles, par lapport aux versions Montague et Bonlac, ainsi qu à la version du Mause-Buch. Mais le fait que l'épisode ln capitaine, qui manque entièrement dans la version Breslau, se retroure intact dans la rersion tatare démontre que celle-ci n'est pas un dérivé corrompn de la version Breslau, telle qu'elle nons est conservée. Les deux versions dérivent donc d'une source commune perdue.

Nous arrivons à la troisieme branche des versions orientales, celle dles Mille. et un Jours.

On sait que, dans la préface de la célèbre édition des Mille et un Jours de Pétis de la Croix (publiée en $1710-1712$ ), il est dit ${ }^{2}$ que cette wurre est la traduction d'mu recueil de contes persan, Hezaryek-Fouz (= Les mille et nu jours), qu'anuait fait, d'après des „comédies indiennes," un derviche Moclès, avec qui Pétis de la Croix avait été „en liaison d'amitié à lspalıan, en 1675, et qui lui anrait alors communiqué son recueil mannscrit ${ }^{3}$. Ces informations conceruant la source des llille ot un Jours ont paru suspectes à plus d'un titre. D'aborl, on ne commaît pas l'existence d'anciennes comidies imliennes, et puis l'auteur de la préface des Ifille et un Jours commet une erreur évidente, quand il dit qu'on roit "à la Bibl. du Roy" une traduction turque de ces comédies indiennes ,sous le titre de Alfaraye Badal-schidda, ce qui signifie la joie après l'affliction"; car le ns. turc, portant ce titre et conservé a la Bibliothèque nationale, ne contient pas des "comélies", mais des contes, qui ne sont même pas iłlentiques i cenx des Hille et un Jours, et il est d'ailleurs comnu qu'on n'a ni coméclies tmques, ni tradnctions turques directes l'onrrages indiens ${ }^{4}$. Si done il fant se garder de prendre l'historiette de la préface des Mille et un Jours à la lettre, il est, d'autre part, évident que Pétis de la Groix n’a las simplement inventé ses contes en imitation

' Ce trait de loiseau indicalcur se retronve dans un conte des Wille et unc Vuits yui présente quelque analogie avec le conte de la femme chast' convoitic pur son benu-fire, mais uni en est historiquement indejenlant: e'est 1 Histoire les Amants de siyric (v. Burton, The Book of the Thonand Nights and a fight [Isyt], $t$, X1, p. 171 et note 2). Cp. aussi M. Lidzharski, Geschichten und Lieder aus ren neu-uramëischen Hss. der Kyl. Bibl. zu Berlin (1896), p. 101 (Ihas Mädchen im Kiasten), et note '2, oì l'auteur signale encore d'antres contes laus lesquels un trait analogue se retrouve.

2 C'est, d'ailleurs, Cazotte qu'on suppose avoir écrit cette préface.

${ }^{3}$ V., dans l'éd. de 1729 , t. I, p'p. $3-6$.

V. W. A. Clouston dans Urig and .Inal. etc., p. 386. 
de cenx des Mille th une Nuits, rendus populaires par la traduction de Galland, mais yu'ils sont vaiment des raluctions, plus on moins enjolivées, de contes orientaux, probablement persans. (In ne comnait pas de Hezuryek-Rouz persan, mais dans l'onvage cité: Alfarage Badal-Schidda (Al Furay Ba'da Alsidda), dont on connaît plusieuts mamiscrits jersans ${ }^{1}$ et tures ${ }^{2}$, quelynes-uns des contes des Mille et un Jours se retrouvent en effet, entre antres notre conte de la femme chaste convoitee par son beaufire. Il serait donc a priori possible que Pétis le la Croix se fît servi, entre autres recueils de contes persans, le celui yni jorte le titre: Al Firay Ba'da Alsidda (La Joie après l'Aftliction).

Comme nous venons de le dire, le recueil persan nommé Al Farag Baida Alsidda contient notre conte, du moins dans la version qu'en donne le ms. Londres, Brit. Mus. Or. 237; dans ce ms. notre conte porte le titre: Histoire de l'Arabe, de sa frmme Ouriga et de son frere ${ }^{3}$. La traduction turque. dans le ms. que nous arons consulté (Brit. Mus. Adl. TS53: El-Ferej ba'd esh-Shiddet, XVII siècle) ${ }^{1}$, donne égalenent notre conte ${ }^{5}$, à jen près sous la même forme. Toici par quels traits la version d'All Faray Pa'da Alsidila, d'après le ms. persan de Londres. diffère de la rersion primitive ${ }^{6}$ :

Personnages: (1) un Arabe; (2) Ĉriyals; (4) un roleur de grands chemins; (5) un eschve négre; (6) un jeune homme yni a ditoume l'argent du roi. Spisodcs: (a) il y a une introduction ou il est question du

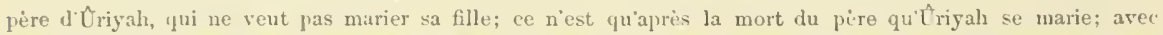
quatre tímoins le beau-frère surprend, dans la chambre d'Ûriyah, un faux amant yu'il y avait fait entrer lui-même; (b) Ûriyah est naltraitée aussi bien par les parents de lenfant assassiné que par l'assassin luimême; (d) le eapitaine du navire est emporté par-dessus bord prar un coup de vent, et Ĉriyah arrive sur le navire it un port de mer, appelé Sahel; (e) le roi dn fıys oủ t̂riyah a trouvé un refuge devient amoureux d'elle, mais est repoussé; son bras ayant ité par deux fois atteint de paralysie et guéri par Ûriyah, iI se convertit ì lislamisme aree tout son penple; ąrés sa mort, triyah devient reine; (f) le jeune homme ra-

1 V. Ch. Rien, Catal. of the Persian Manuseripls in the British Musem, II 1881), 1). 559 (MIs. or. 237); 1. Chaurin, Bibliogr. des outrages arabes. IV (1900), p. 126. Dejà en 18:2, W. Onseley (Trarets in rarious coun-

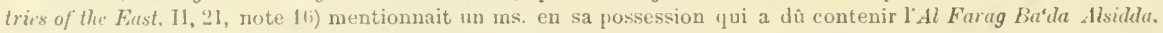
puisque lhéroine de notre conte $\mathrm{g}$ porte le mème nom (Aruiah).

$=\mathrm{V}$. Ch. Rieu, Catalogue of Turkish Mamusripts in the British Musezm (1853), pp. 201-5 (ddd. 7843); V. Chauvin, Biblingr, des ourrages arabes, IV (1900), p. 1:66.

${ }^{3}$ V. (3. Rien, Calnt. of the Persian MLs. in the Brit. Wus., II (18s1), 760 a: Ms. or. 237, fol. 117 b: The Arat), his wife trigah. and his brother. Le ms. date du XVI:e ou du commencement du Xlll:e siècle; commo auteur est nommi MIuhamned Käzim B. Mlìak Ilusain Muzaffarî Sujâvandî, poetically surnamed Hubbi (v. Hieu, onvr. cile, 1). $75 \%$ ).

- Ln des unss. de cette fraduction turyue, conservé it la Bibliotliéque nationale de Paris sous la cote "f. or. anc., ture $377^{\circ}$, date des environs de 1 amnce $14 x() ; \mathrm{r}$. W. A. Clouston dans (iriginals and dnalogues of

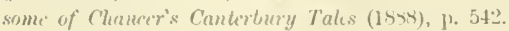

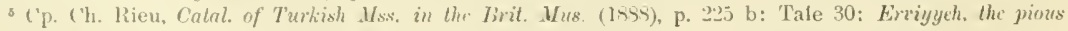
unman. whom her lusband's brother attempted to sciluee (f, 194 a).

- Nous nous sommes servi d'une tralnction auglaice que M. Denison lioss, professeur de langues orientales ì l'liniversiti de Londres, a eu l'obligeane de nous procurer. Nous en lomons une traduction française daus notres Aprendire (B). 
cheté est accompagné de sa mère; (g) le mari, le voleur, la mère du jeune homme et le capitaine racontent d'abord pourquoi ils sont renus; le lendemain, le capitaine. le jeune homme rachetr, le nègre et le beaufrẻro se confessent; pour éprouver la filélitè dı mari, t̂riyah lui offle, avant de se fairc reconnaitre, une de ses belles compagnes pour femme; îriyah et son mari règnent longtenys ensemble et se retirent ensuite dans la solitude pour servir lieu, laissant le gourernement au frère.

Par le trait que l'héroïne devient reine, trait trop exagéré pour ponvoir être primitif, cette version se rattache à la version Breslan de la brancle des Mille et une Nuits, mais représente en même tempss, si l'on exeepte quelques actails, nne version beaneonp moins éloignée de la version primitive.

Nons avons déjả dit plus haut (1). 18) qne notre conte se retronve dans le célèbre recneil de contes intitnlé Mille et un Jours; il y porte ce titre: Histoire de Repsima ${ }^{1}$. Il entre naturellement anssi dans les nombrenses tradnetions des Mille et. un . Jours ${ }^{2}$. Chose plus eniense, l'Histoire de Repsima a en la bonne fortmie de devenir „livre populaire" en Suède. La plus ancienne édition connue est celle de Hernösand de l'amnée 1802, portant le titre: Den Sköna. Repsimas Besynnerliga Händelser; c'est une traduction presque littérale llı texte de Pétis de la Croix ${ }^{3}$.

La version des Mille ot un Jours diffère par les traits snivants de la version primitive $^{4}$ :

Personnages: (1) Temim, marehand de Basra; (2) Repsima; (3) Revendé; (4) un voleur de grands chemins; (5) Calid, nègre. Épisodes: (a) une introduction comme dans la version d'Al Farag Batda Alsidda: le père s'appelle Dukin, et c'est Repsima elle-même 'qui ne veut pas se marier; la scène du flagrant délit comme dans la version d’Al Farag Báda Alsidda: Repsima est enterrée vivante jusquà la poitrine; (e) Repsima arrive ì une île, gourernéc par une reine, oil los labitants lui donnent une retraite; elle accuiert

1 Nous n'arons pas vu l'édition princeps (1710-1712) des Mille el un Jours (i la Bil)liothèque nationale de Paris il n'en existe qu'un exemplaire dépareillé, oú manıue le volıme contenant l'Histoire de Repsimu). Dans lëdition de 1729, te conte se lit t. V, pp. 241 - 95 (jours 947-1001); dans l'édition de 1526, t. 11I, pp. 117-53 (jours 21S-232). V. encore Le Cabinet dés fées (Amsterdarn 1785), XV, 431-65; (Cienève 17×6). XV, 477-514; Panthéon lithérairc, Contes orientaux, Il (1835), 265-74; éd. de k', de Donville (Paris 1883), pp. 357-84. Pour la bibliographie des éditions (et traduetions) des Mille et un Jours, v. V. Chanvin, Bibliographie dt's ouvruges arabes, IV (1900), 123-32.

2 V. en anglais: King, The Prrsian and the Turkish Tales, If (171t), 7001-26 (The Story of Repsima); A. Philips, The Thousand and One Trays (3:0 éd. 1722). 1II, 292-330 (The Hislory of Repsima); H IVeber, Tules of the East, II (1812), 517-25 (The History of Repsima, d'apres Philips); The Persian Tales. or The Thousand and one Trays (Bombay 1S48), 11, 101-11 (The Stmy of Repsima; cette édition est faite daprès celle de King, réimprimee en 1824); J. H. Mc Carthy, The Thoustend and One Ltays (IS92), II, 208- t2 (IX. The Story of Repsima; il y a au commencement du volume une gravure de Stanley L. Wood, représentant la scène où le nègre déclare son anour à Repsima); en allımnd: Tausend und ein Tag (1745), pp. 862-93 (Hislorie von (ter Repsima); F. H. von der Hagen, Touscnd und tin Tag. IV (1827), 192_23I (Geschirhte Repszimi's). Dans la Bibliogruphic de Chauvin (v. la note précédente) ne sont pas indiquées quelques-unes des traductions que nous counaissons: celle de Jíng, celle de Philips de 1722 et Ia traduction allemande de 1745.

3 Cette édition fut signalée en 1815 par P. O. Bäckström dans ses sivenska Folkbörker, 1, 270. Deux autres éditions sont mentionnées par H.j. Linnström, Svenskt Boklexikon (1883-1564), 1, 64t (No. 152: Ein underbar Historia om den sköna Repsime. Hvari visas att dygden till slut belönas, men att laster och brott alltid blilva straffade. Ganska nöjsam att lisa. Vestervik 185t. 16 PL.), et II, 29.t (Repsima. Samling af sagor till nöjsamt tidsfördrif, no. 1. Vestervik 1859. 24 pp.).

- Nous nons sommes servi de l'údition de 1739 des Mille et an Jour (sir). 
l'ustime de tout le monde, la reine la choisit pour son héritière, et apres la mort de la reine Repsima monte sur lo trone; (f) le voyage $d+s$ six hommes n'ust pas raconté: un beau jour, ils se trouvent en même temps devant la porte de Repsima; (g) le mari, le voleur, le capitaine et le débiteur racontent d'abord pourquoi ils sont venus, aprés quoi tous sont renvoyés; le lendemain, le negro te le beau-frère se confessent aussi, et les criminels, en dernier lieu Revendé, sont guéris; conme taus la version d'dl Farag Báda Alsidda, Repsima offre it son uari une notivelle femme; Repsima retient son nari avec elle, et Revendé devient son premier ministre.

Comme on le voit, les divergences de l'Histoire de Repsima l'avec la version d'Al Faray Bu'da Alsilda ne sont pas très grandes yuant an fond lu conte, de sorte quion pourrait être tenté d'admettre gne Pétis de la Croix a tout simplement pris som histoire dams le recueil de contes persan. Il y a cependant un épisode dans la version des Jille ot un , Jours quil faut, grâte an témoignage de la version Montagne des Mille el une Vaits et de la version tatare, regarder comme plus primitif une l'épisode correspondant dú recueil persan: e'est l'épisoule de la tempête. II fant done rroire que l'étis de la Croix n'a pas utilisé le recueil Al Farag Báda Alsidda, du moins dans la forme que nous connaissons, mais qu'il s'est servi d'un antre recueil de contes, leut-être raiment m Hesuryel limz perdu (v. ci-dessus p. 18), d'oi notre conte serait remu anssi daus le recneil Al Furag Ba'da Alsilda. Ce reneil de contes perdu, Hczaryck: Rouz on antre, était pent-être une imitation persane les Hille ct une Nuits, composée de contes de provenance arabe ${ }^{1}$. Comme notre conte, dans les rersions d'Al Faray Bu'da Alsidila et les Mille et un Jours, a cela de commun avec la version Breslan des Mille et une mits et la version tatare que l'héroüne devient reine, il est possible que la source ale l'Histoire de Repsima ef lu conte du recneil intitulé Al Faray Bu'da Alsidlu ait en une origine commme avec la troisième version arabe. Dans tous les cas, le commencement des denx versions dont nons nous sommes ocempé ci-dessus n'est certainement pas primitif, car il est tont ì fait imutile à la marche du récit que l'hérö̈ne ne se marie pas avant la mort de son père. Ce commencement a sans doute été ajouté plus tar'd pour renforcer l'idée de la grande chasteté lle l'héroüne². L'introdnction d'un faux amant dans la chambre de l'héroün doit également être regarlée comme un trait postérienr, car le rôle des faux témoins derient par là suprerfu. Eulin, la scène de la confession, avec tsms ses retards, a bien l'air d'ôtre altérée; l'orilye du récit semble demander que les visiteurs racontent successivement leu histoire, en (ommençant par le mari et en terminant par le capitaine (c\%. lil version Montague).

Cp. IV. A. CHonston dans (Mriginals and Analogues ete. (1888), p. 542.

2 A rause du nom de lihíröne, Ripsima, dans les Mille el un Jours, on pourrait être tentè de voir, dans l'aversion de l'héröne pour le mariage, un écho éloigné de la legende arménienne do sainte Rijsime

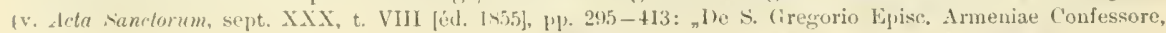
item de s. Virginibus Ripsime, Gititna et soriis martyribus in Armenia Majore"; cp. S. C. Malan, The Life 
Il nous reste ì parler de quelques autres versions appartenunt ì la troisième branche des versions orientales.

Il fant tont d'abord mentionner nu drame en trois actes, basé sur l'épisode dı meurtre, d’après les Mille et un. Jours de Pétis de la Croix: liepsima, essui d’une trugédie domestiqne, publié à Lausame en 1767 , sans nom d'antem ${ }^{1}$. Le rérit a naturellement été un pen changé:

Lution se passe dans la tente du voleur, appelé Adel. Celui-ci est aussi amourenx de Repsima, ce qui provoque la jalousie de sa femme, Zamé. Calid ne tue pas l'enfant; il l'emporte seulement et tue it sa place un cherrean. Temin (sie) retronve sa femme dans la tente d'Adel, après avoir appris son innocen'e the la bonche de son frère mourant. Calid est tué par un coup de foudre.

Il est probable que l'Histoire de Repsima a aussi domné naissance ì me pastorale basquè ${ }^{2}$, La princesse de Cazmira, composée de 6492 vers, répartis en 206 rôles. D'après mne analyse donnée par M. J. Vinson dans son onvarge intitulé Le folk-lore du pays basque $(1883)^{3}$, la version basque diffère par les traits suivants de la version primitive:

I'ersonnages: (1) le fils du roi cle Chiraz; (2) la prineesse de Cachemire, fille du roi Torgul; (3) Valen; (t) nn Mlaure; (5) = (4. Lisisodes: (a) l'héroüne est jetée dans un tron par Valen; (b) elle poignarde le Manre, 'qui reut la prendre do force, après quoi elle s'enfuit; (d) le capitaine la dépose dans nne île; (e) elle devient reine; (f) l'analyse ne dit rien sur le voyage des criminels; ( $g$ ) l'analyse dit senlement qu'elle retronve son mari.

Le trait que la princesse devient reine montre évidemment un rapport étroit avec la version des Mille et mn Jours. Ia pastorale hasque est sans donte basée directement sur le conte de Pétis de lis Croix.

Il est plus difficile de dire si mu conte populaire gree, recueilli en bipire (Janina) et qui se rattache de près au groupe des lille et un Jours ${ }^{4}$, a été inspiré par le conte de Pétis de la Croix, ou bien s'il est veun directement de l'Orient, peut-être par

and Times of S. Grryory the lluminator [1868], p]. 1\$7-213). Cette vierge romaine tres belle, qui anrait vécu i Rome sous l'empereur Dioclétien, s'était vouée au célibat. Comme l'emperenr voulait l'éponser, elle s'enfuit avec ses compagnes jusqu'en Arménie. Lda, le roi Tiridate veut la violenter; elle sort victorieuse de cette épreuve, mais doit subir le martyre. - Il est cepenlant probable que c'est Pétis de la Croix qui a domné des noms aux persomnages du conte. Autrement, on ne comprendrait pas bien pourquoi, dans le recneil Al Farag Báda Alsidda, ancun des personnages, excepté l'hèroïne, n'est nommé.

1 L'autenr de cette tragédie domestique était M:lle Bonillé, fille d'un réfugie d'Amsterdam, morte ¿L Lausanne en 1816 (r. A.-A. Barbier, Rictionnaire des ouvrages anonymcs, 3:0 éd., t. IT [1879], p. 323 a: „Repsima“ - - -). M:lle Bouille dit elle-même (p. 3) que le sujet de sa piéce „est pris des Mille d: un Jour.s, Conte's Persans, Tome VII, dans le Conte intitulé Histoire de Repsima." Elle donne un résumé de ce conte jusqu'i l'épisode du meurtre inclus (pp. 3 \%).

${ }^{2}$ On sait que les pastorales basques sont des pièces de théatre, comparables aux anciens mystères français et qui sont jotées encore de nos jours (v. J. Vinson, Le Folli-lore du pays basinue [16s3], p. 32t).

${ }^{3}$ V. pp. 344-5. C'est M. Kr. Nyrop, Storia dell'popea francese nel medio evo (trad. de M. Eg. Gorra, 1596), p. 211, note 3 , qui a le premier signale le rapjort existant entre notre conte et la pastorale basque.

4 R. Köhler, dans la Jenaer Litteraturzeitung, annèo 1878, p. 306, avait déjà signalé le rapport étroit 'qui existe entre le conte grec et l'Histoire de Repsima. 
lintermédiaire d'une tradnction turuge ${ }^{2}$. Nons sommes porté à croire a la première alternative, rn l'utroite ressemblance, pour plusieurs détails (p. ex. le fait que l'héroüne succide it une reine), entre l'Histoire de Repsima ef notre conte. Il est vrai que le conte grec décrit le royage des six hommes it la résidence de lihéröne, ce qui a tont l'air d'être un trait primitif, tandis que le conte de Pétis de la Croix ne le fait pas. Mais il ne semble pas trop osé d'ahmettre que c'est le conte grec qui a changé, à cet endroit, la version re sil source, celle-ci étant quelque peu artiticielle.

Le conte gree fut publié la première fois par J. G. von Haln dans ses Griechische und Albanesische Märchen (1864) sous le titre: Von der Fruu, die Gutes thut und Undank erfährt ${ }^{2}$. Tue conte diftère par les traits suivants de la version primitive:

l'ersonnagcs: (1) un marchand grec; (4) un voleur niggre; (5) un autre voleur. Episodes: (a) il y a une introduction qui tend à démontrer le caractére dissipó du beau-frère et le caractère généroux du mari; le beau.frire introd’it un homme ivre dans la maison du frére pour convaincre le cadi de la vérité de son accusation; la femme doit être mise à mort, mais les bourreaux se contentent de l'enterrer juspu'au cou; (d) tout l’équiparre est sauvé dı naufrage; $(\Theta)$ = la version des Mille et un Jourg; la nouvelle reine fait bâtir un grand hôpital pour les malades qui viennent chercher guérison; (g) seulement les criminels racontent leur histoire; tous restent aupries de la reine et reçoirent des cliarges à sa cour.

Nons avons ainsi passé en revue tontes les versions de la branche orientale de notre conte que nons connaissons ${ }^{3}$. Voici, en guise de répétition, comment nous nous en figurons la filiation:

1 En aucun cas, le conte grec ne peut venir d'un roman français perdu, comme l'ont supposè quelques savants; v. Iuulop, Ilistory of prose fition (éd. 1848). t. I, 1. 91, note; E. Rohde, Der gricrhische Romun (1576), 1). 534, note 2; 2:e éd. (1900), p. 568, note 2; B. Sichmidt, Griechische Marchen, Sagen und Volkslieder (Iธร7), p. 7 , note 1 .

3 V. t. I, pp. 140-8 (No. 16). Plus tard (en 1879) il fut publié en grec par J. Pio dans son ourrage

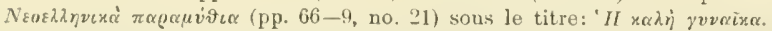

3 Wapres E. Rohdo, Ther griechische Roman (1876), p. 534, note 2 (2:e ed. [1900]. p. 569, note 2), deux autres contes orientaux provientraient encore de la source indienno de notre légende: Aienlures de la fille drun Vizir (v. Cardonne, Mil. de litt. orient, t. II [1770]. pp. 3(i-57) et L'hroïsme de la pieté filiale (v. Abe]Rémusat, Contes chinois, t. 1 [1827], pp. 3-129). Bornons-nous ì dire yue ces contes ne nous paraissent avoir aucun rapport historique avec notre légende: ni dans lun ni dans l'autre ne se retrouvent les traits caractéristiques du conle de la femme chaste convoitée par son beau-frère. D'ailleurs, le conte qu'a publie Cardonne n'est ¡u'une variante du conte albanais qu'a publié G. Mleyer dans Areh. für Litt. gessh., t. XII [1854], pp. 127-132: No. 11. Ihas Müdchen im hasten, et dont nous avons parlé ci-dessus p. 3, note 1 (sous 3). 


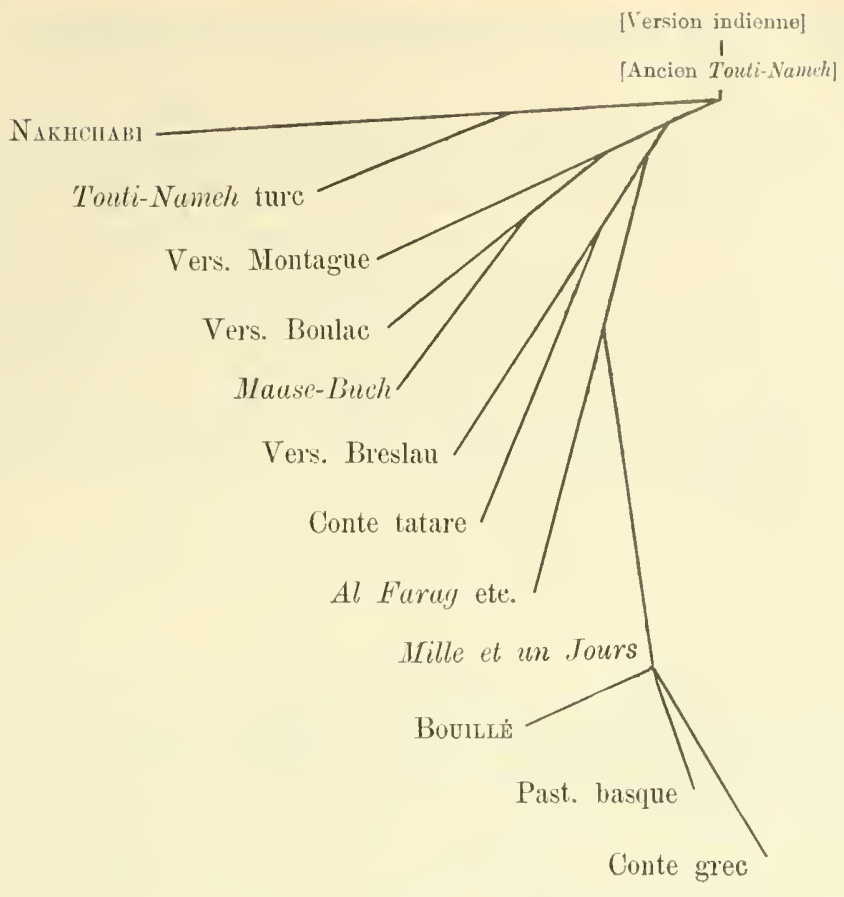

CHAPITRE IV

\section{Les Gestu Romanomm}

Comme il a déjà été dit dans le chapitre II (11) 8-9), toutes les versions occilentales du conte de la femme chaste convoitée par son beau-frère ${ }^{1}$ présentent en commu un trait important, l'emprisonnement dn bean-frère, qui prouve que ces versions proviennent d'une source commune, version orientale quelconque. Ln antre trait de moindre importance, bien que non sans valeur, est que le mari est tonjours un très haut personnage, empereur ou roi. C'est donc avec ces denx traits supllémentaires que notre conte a été apporté en Occident, et le fait qu’il se rencontre sons une forme très abrégée

1 Par le nom de versions occidentales nous ne désignons aturellement que celles des versions en langues européennes qui n'appartiennent pas directement au groupe des versions oriendules. Ainsi p. ex. la pastorale basque (v, p. 2l) n'est pas une version occidentale, non plus que le conte grec (v. pl, 2l-2l). 
dès le milien du XII" siercle fait suproser que son introduction en Europe ne peut guère être postérieme à la fin du XI ${ }^{\mathrm{e}}$ siècle.

Deux des groupes occilentaux que nous aurons à traiter, celui des Gesta líomanoram et celui de Florence dr fiome, se rapjochent davantage. pour le fond du récit, de leur source orientale: ils ont tous les deux conservé les quatre rriminels (le beanfrère, lassassin, le jeune homme lacheté et le capitaine). Il $\mathrm{y}$ a done lieu de croire qu ils forment entre enx une branche a fart, frovenant dime source commune. Ancum de ces denx groupes ne pent cependant dériver directement de lamtre, chacun ayant conservé des traits primitifs qui manquent dans l'autre. Si nous parlons d'abord du groupe de versions que nuts appelons celui des Gesta Romunorum, r'est parce que celui-ci, à tont prendre, s'éloigne moins de notre version primitive (indienne) que celui de Florence de Rome.

Des Gesta Romanorum, le célébre recueil latin de contes moralisés, compilé à la fin du XII ou an commencenent du XIV siècle ${ }^{2}$, il existe denx rédactions principitles: l'me d'origine anglaise, l'autre d'origine continentale, dont celle-là est probablement la plus ancienue ${ }^{3}$, mais dont les rapports exacts n'ont pas encore été définitivement fixés. Notre conte se retrouve, arec de fort légères différences, dans les deux rédactions. Toici d'abord par quels traits ces deux versions en commun diffèrent de notre version primitive (inclienne):

Personnages: (1) un empereur de Rome; (4) un grand seigneur; (5) un sénechal au service du sejgneur; (fi) un malfaiteur. Épisodes: (a) l'empereur, yui part pour la Terre sainte, laisse le gouvernement i sa ferume; limpératrice, obsedé des déclarations d’amour du beaufrére, le fait jeter en prison; à la nouvelle tu retour de l'emperenr, l'impératrice, accompagnée du beau-fríre reliché $\theta t$ d'une nombreuse suite, se rend ì sa rencontre; en route, comme la suite commence it courir apris un cerf, le beau-frére veut faire violence à l'imperatrice; ne parrenant pas à ses fins, il labandonne dans la forkt, attachée par les cheveux i un arbre, et va raconter à son frìre que l'impératrice lui a été enlevie par une troupe d'hommes armés; l'enfaut que limpératrice doit soigner est une fille; (b) le conteau est placé dins la main de l'imperatrice, dormant à côté de la fille; cest la femme lı seigneur yui accuse l'impératrice dı uneurtre et veut la faire turr; il n'est pas yuestion l'argent de voyage; ic) le malfaiteur racheti devient le serviteur de l'impicratrice et ne tombo fras amoureux a'ule; c'est le capitaine qui, ayant vu la belle imperatrice, corrompt le serviteur;

1 Dans la Kraserchronik, 'jui a cité écrite environ 1150 (v., pour cette late, Die Kaiserchronik, il Edw. Sichröder, 159?, p. 44).

* V., pour cette date, Gesta Romanorum, éd. H. Österley (1872), p. 257.

3 V. Giesta Rom., éd. Österley, pl. 262-6.

4 F. Liebrecht (The Acadcmy, 1V, IS73, col. 464) caractirise ainsi le rapport des deux redactious: ,it is clear that the supposition of an independent Anglo-Latin text is untenable, and that the English version can only be counted as one of the branclues of a widely suread family of manuscripts". 
l'impératrice est enlevée, quand elle va acheter des marchandises sur le navire; (f) les veyagenrs, parmi lesquels n'est pas le maitre de l'assassin, ne se rencontrent qu'au couvent; (g) le beum-frive ne confesse son crime quaprès une seconde sommation.

La rédaction anglo-latine de cette rersion n'a pas encore été imprimée. D'après les unss. que nous avons consultés ${ }^{1}$, elle présente les traits caractéristiqnes suivants:

Personnages: (1) Menelaus ${ }^{2}$; (2) la fille du roi de Hengrie. Yipisodes: (a) il y a une seconde raison pourjuei l'imperatrice fait jeter lo beatu-fère en prison: c'est qu'il s'immisce dans les aftaires publiques de l'empire; (c) dans la ville ou l'impératrice loge avec son serviteur, ce n'ust pas seulement le capitaine qni siprend d'amour pour elle: beaucoup de personues le font.

De bonne heure, la rédaction anglo-latine des Gesta Romunornm fiat partiellement traduite en anglais. De cette traduction anglaise on possèle aujourd'hui quelques mss. des $\mathrm{XIV}^{\mathrm{e}}$ et $\mathrm{XV}$ e siècles ${ }^{3}$, dont un (Londres, Brit. Mus. Harl. 73333, XV siècle) donne anssi notre conte sous le titre Merelaus the Emperour (chap. 699). En 1838, cette version anglaise de notre conte, qui ne diffère guère pour le contenu de la version latine, fut imprimée par Sir F. Madden dans sa magnifique édition des Gestu R'mnanorum anglais ${ }^{4}$. Mais déjà trois sièeles anparavant, notre conte avait été imprimé en anglais dans l'édition des Gesla Romanorum de Wynkyn de Worde (vers 1510-1515) sons nne forme quelque pen différente ${ }^{5}$. Daus cette érlition, qui ne contient „u'une partie (43) des histoires originales, notre conte, qui occupe la quanantiène place, domne

1 Ce sont les mss. Londres, Brit. Mus. Har]. 2270 ( $\mathrm{XV}$ :e sicele), chap. 101 (fol. S1) $\mathrm{r}^{0}-82 \mathrm{v}^{0}$ ), et Londres, Brit. Mos. Harl. 5259 (XV:e siecle), "lap. 99 (fol $117 \mathrm{r}^{0}-120 \mathrm{r}^{0}$ ). Nons donnons in extenso le texte du premier de ces mss. dans notre Appéthlie (C). - Cette version latine a cité racontie en anglais par Fr. Douce dans sa Dissertation on the Gesta Romanorum, incorporée dans ses lllustrations of shakspeare and of Ancicul Manners (1:ère ed., [४07, t. II, pp. 416-20; éd. 1839, pp. 568-70).

${ }_{2}^{2}$ Dans les mss. Londres, Brit. Mus. Harl. 5259 et 5369 (XV:e siécle, chap. 41); v. Gesta Rom., éd. (̈)sterley, 1) 192), il y a, par la mejrise d'un enlumineur queleonuce, Gerelaus (le nom ne s'y tronve qu'une fois, comme le premier mot de la version).

${ }^{3} \mathrm{~V}$. jour ces mss., Gesta Roman,, éd. H. Österley, pp. $237-41$.

+ V. Sir F. Marlden, The old english version of the Gesta Romanomum, p1, 251 - 60. I tans la rëimpression diplomatigue de ees Gesta par 11. S. J. 11. Herrtage pour The Early English Text Socicty (The Eirly English Versions of lle Gesta Romanorum, 1879), le conte (Nerelaus fie emperour) se trouve lp. 3H-9. La même version a eité réimprimée par I. F. J. Furnivall dans les Orig. and Anal. of some of Chancer's Cantorbury Tules (1854; cette partie imprimée en [872), 1'p. 57-70, sous le titre: The Tale of the Wife of Merelaus the Enperor.

${ }^{5}$ Lion ne connait yu'un senl exemplaire do cette édition, conservẻ ì St. John's College ì Cambridge; v., sur ce livre, The Retrospertive Review, t. II (1820), 1p. 327-9 (cet article a ité reproduit par C. H Hartshone, The Book Rarilies in the University of Cambridge [1829], pp. 395-400), et F. Maduen, ouvr. cite. p. XV, note *. On a supposé que l'auteur de cette édition fut John Leland, antipuaire (mort en 1552), supposition émise par Richard Robinson dans son eurieux ouvrage Fupolemin, Archippus \& Panoplia, encore inédit (ms. Londres, Brit. Nus., Reg. 18 A LXVI), ecrit en 1603. Dans cet ouvrage, sorte de catalogue des livres imprimés de Robinson, de ses patrons et de leurs bienfaits, on lit (fol. $5 \mathrm{r}^{0}$ ): „1577. A Record of Ancyent Historyes. intituled in Latin Gesta Romanormm translated (auctore vt supponitur. Johane Leylando Anticuario) by mee perrsed corrected and bettered" (le passage a lejji été donnes jar l'r. Donce, Illustr. of shakspeare, $t$. 11 [1507], pp. 424-5, avec 'puel(gues clangements insignifiants dans lat transcription). Cp. repenuant l'attitule réservée do F. Madden, ouvr. rile: p. XVII, note". 


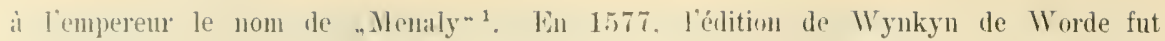
réimurimée pal Richard Ruhinsun aver des corrertions et les additions ${ }^{2}$, et rette édition

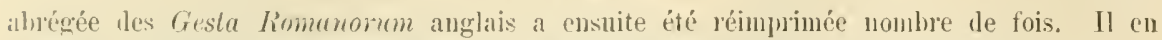
existe des élitions des XVI". XVII" et XVIII" siecles dans deux rédactions différentes ", et en 15et Ch. Swan, daus I'Jutroluction de ses Gesta Romanomum, réimprima notre conte d'apres l'me de res ressions'. Ajontons encore que le poite anglais Thomas Hoceleve on Occleve (environ 1:3(is-1fio) a fait son conte en vers anglais, intitulé Fubula de quatum Tmperatrice lomuna, d'ajorès un des miss. latins où, par la méprise de lenluminenr. .. Ventslaus" est devenu ,fereslans".

Les diffórentes versions montionnées de notre conte ne présentent que des divergines de peu d'importanee.

A côté de la version anglo-latine et de ses dérives anglais, il existe me version rontinentale. Cette version fut pour la premiere rois pulliée en 1872 par H. (Osterley Lans son érlition des Gestu Romanmum ${ }^{6}$. Plus tarrl (en 1890) elle a été publiée,

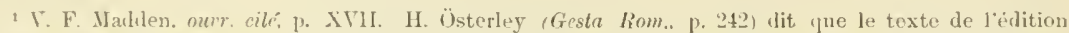
de Wynkyn de Worde est une tralustion fidele du ms. Brit. Wus. Harl. 536\%, mais cela doit etre une erreur, puisque Pempereur y est appele „Gertaus" (c1. F. Madden, ouvr. cité, p. 521, note au chap. LXLX).

2 ("est l'édition, actuellement introur:able. dont, il a èté question ci-dessus 1). 25, note 5.

3 Nous avons ite it même 3 e consulter une édition de 1648 (Londres), portant le titre; $A$ Rerord of Anrient Historips. Entituled in Latine: Gesta Romanorum. Now newly Perused and Correcterl. with some thing alded, by R. R.", ainsi que les iditions de 1663, 1659, 1696, 1698, 1703 et 1713 . Dans ros didions, on l'empereur porte le nom ae , Mlenelay", notre conte est tonjours to flieme, mais le nombre les rontes y est porti it 4 . Douke (llustr. of shaksperare [1507], t. 11, pp. 4.7-8) mentionne encore des iditions le 1595,1665 et $167 \%$, ainsi yte denx sans date (imprimcies l'une par R. Bishop, l'autre par Stanshy). Madiden couvr. eite, p. XVIII et p. XIX, note*) y ajoute tes iditions de 1610, 1715 et 1753 ; il suppose en outre que I'edition sans date inprimce par Stanshy est de 164:3. Dans Ilazlitt, Hand-Booli to the Pop., Poet., and Iram. Lil. of Gimeat Brituin (1867), p. 227, et Seend Ser. of Bibl. Coll. and Sotes on Early Engl. Lit.

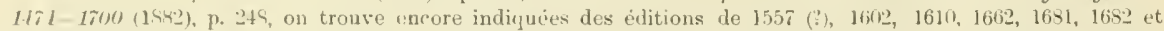

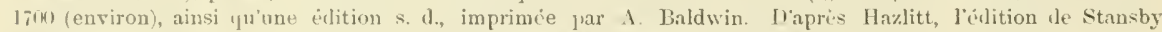
daterait denviron 1620, celle de Bishop d'environ 1640. Robinson lui-même a dit, dans l'ouvrage intitulé Eupolemin, Archippes of I'anoplia (ms. Londres. Brit. Ius., Reg. 18 A LXVI, fol. $5 \mathrm{r}^{0}$ ), yue sa version des Gista limanorum fut imprimée six fois à partir de 157 jusquen 16(01. Donce (ouvr. eité, p. 427) nous informe que l'idition de 1545 ne conteuait encore "pue 43 contes, comme celle de Wynky de Worde; ce n'est pro-

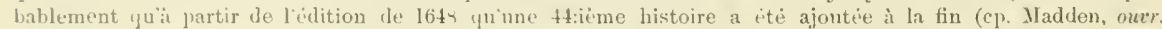
rite. P. XIX). La seconde redaction anglaise des Gesta Romanorum. qui contient 54 listoires, donne à

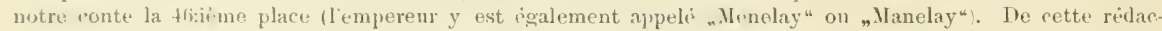
tion nous ne connaissons que les deux editions suivautes: Fiesta homanornm: or Fifty-eight Histories (1rigi-

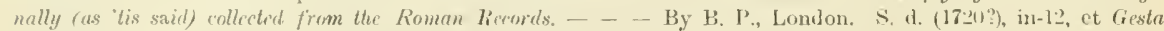
bomanomem: containing Fifty Eight Remarkable Histories, Collected originally from the bist and most aneient Roman Rererds. - - By A. B., London 172:, in-12.

\& T. Ch. Swan, Festa Rum., t. I, pl. CXIV-CXXXII (chap. (I); ed. de 187t, publiee par W. Hooper

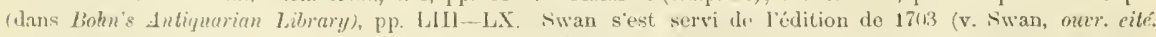

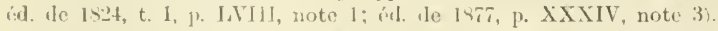

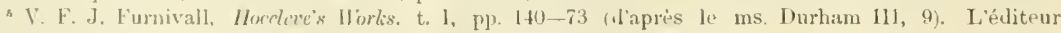

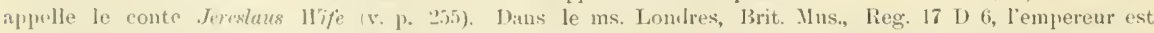
nommé ,Gereslans" (v. Furnivall, our. citr; t. I. P. 255).

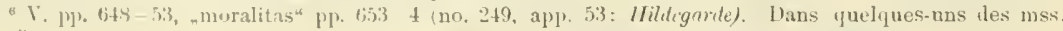
vitis par Österley, notr. légende est munic dune moridur: tels les mss. Jreste, C, 14:3 d (env. 1422): Ihe 
d'après d'autres mss., par $W$. Dick dans son édition du célèbre recneil d'exemples ${ }^{1}$. Cette version se distingne par les traits suirants:

Personnages: (1) Octavianus. Épisodes: (a) après avoir abandonné l'impératrice dans la forêt, le bean-frère reprend simplement le même chemin par où il ètait venu²; (e) l'impératrice apprend les vertus médicales des plantes, de sorte qu’elle guérit les malades d'nne manière tonte naturelle; (g) l'impératrice n'a pas de voile, elle tâche seulement de ne pas faire voir son visage; anjès la première confession incomplète du beau-frère, le reméde est essayé sur lui, mais sans résultat.

De cette dernière version latine il existe deux tradnctions différentes en langue allemande. L'une d'elles fut publiée la première fois en 1757 par 'l'h. J. Bodmer dans l'onvrage intitulé Fabelu uns den Zeilen der Minnesinger (de Boner') ${ }^{3}$ d'après m ms. dn XIV ${ }^{\text {e }}$ siècle $^{4}$, la seconde fois par H. F. Massmann, en 1854, dans son culition de la Kaiserchronik ${ }^{5}$ d'après nn antre ms. pell différent. Dans cette dernière rélaction notre conte porte le titre: Ton octuviuno dem ehaiser des fraw so vil vmb ir rainigehuit laid, so dz sy irs manns brider vieng und in darnach wid' ausz liesz etc. ${ }^{\circ}$ L'antre traduction allemande a été donnée par J. G. Th. Grässe, en 184:2, dans le premier appendice de son élition des Gesta Romanonum d'après le ms. Burlin, Grimm. 81, écrit en $1469^{7}$. Ces denx versions de la rédaction allemande ne diffèrent entre elles que pour des détails insignifiants.

Ayant ainsi passé en revue les denx versions principales des Gesta Romanorum, l'anglo-latine et la continentale, avec leurs traducticns anglaises et allemandes, il ne nous reste gu'ì constater que le nou de l'empereur, aussi bien Menelaus qu'Oetavi-

Octaviano qui super omnia uxorem dilexit (v. Österley, ouvr. eité, p 25), et Colmar. Issenhem. 10 (XIV:e siècle): Ihe imperatrice que ligno erat suspensa el post diversa perieula al majorem honorem est ratucta (v. Österley, ouvr. cite', p. 181).

1 V. W. Dick, Die Gesta Romanorum, nach der Innsbrueker Hs. vom Jahre 1312 und vicr Mïnchener Hss. herausgegeben, pp. $111-8$ (cap. 150: De fratre imperatoris, qui concupiuit imperalricem et suspencht eam viuam). Sur un ms. de la Bibl. Univ. de Buda-l'sstl (cod. 25, de l'année 1474), oì notre légende oceupe la 134:e place (fol. $69 \mathrm{a}^{1}$ ), v. L. Katona dans Zcitsehe, für vergl. Lill. gesth.. Nene Folge. XI1I (1899), 470-91.

2 Cela est évidemment une altération de la version primitive des Gesta Romanorum; pour pouvoir rester auprès de l'emperenr, il faut que le beau-frére explique de 'quelıne manière la disparition de l'impératrice.

${ }^{3}$ Pp. 262-71 (App. XII).

+ Le ms. Zurich C, 113 (no, 103). Ja rubriipue de notre légende y est: Ton dem laxiser octaviano dez weib alz vil laid umb ir kaüsch so daz si irs mame's bruder hieng (v. Ósterley, ouvr. cité, p. 211).

s. T. III, pp. 913-6.

- Nous avons encore pu voir une traduction manuscrite, presuue identiqne à celle publiée par Bodmer, dans le ms. Londres, Brit. Mus. Adll. 10291 (fol. $83 \mathrm{r}^{\circ} \mathrm{b}-87 \mathrm{r}^{0} \mathrm{a} ;$ no. 1(1:3), daté de 1420. Le titre en est: I'on dem claiser Otlauiano dez weib als vil vmb ir cheusch lod so daz sey - — (mot illisible) man pruder ling. Dans le registre du $\mathrm{ms}$. on lit: Von dem chaiser otlaviano vad seinem weib di sein prul' hing.

? V.J.G. Th. Grässe, Gesta Romanorum, das älteste Mährch'n- und Legendenbuch des thrisllichen Mittelallers (1842), II, 152-9 (Achte krzählung: Vom Kaiser Octavianus). Notre conte occupe dans le ms. la 36:e place et y porte, d'après Österley (ouvr. eite, p. 229), la rubrique: Octavianus lut scin weib lieb, ete. ("est naturellement par erreur que Grässe dit (t. II, p. 281) que la version en question n'est iue l'histoire très simplifiée de l'Empereur Vctavien (appartenant au type de la méchante belle-mère), erreur déjà relevée par Koberstein dans son Grundriss der ciesch. der d. Nat. lit. (v. la 5:e éd, 1si2, 1, 399: \$ 16s, note 22). 
anus, ne pent être rattaché à aucun personnage historique et qu'il est, par conséquent, entièrement fictif ${ }^{1}$.

\section{CHAPITRE V}

\section{Florence de Rome}

11 a déjà été dit dans le chapitre précédent ( $p .24)$ que la branche des Gesta Romanorum et celle de Florence de lome sont apparentées de près. On sernit même tenté de croire que celle-ci dérive directement de celle-là, si la hranche de Florence de Rome n'avait gardé un trait primitif qui manque dans les versions des Gestu Rommorum: l'assassiu est accompagné, chez la sainte femme, du père de l'enfant tué, legnel raronte, à son tour, re qu’il sait concernant la femme persécutée. Il faut ronc admettre que les deux branches dérivent séparment d'une version perdue où l'hôte miséricordienx accompagnait l'assassin. Ia branche de Florence de Rome, tont en se lasant sur ce fond commun, l'a beancoup développé et altéré en certains détails.

Les versions qui constitnent la branche de Florence de Rome sont les snivantes: 1) un roman d'aventure français du premier quart du XIII ${ }^{\circ}$ siècte, en 6+10 vers

i rest peut-ître it la version des Cesta Romanorum de notre conte que le moine anglais Nirholas Tricet (mort apres 1334), anteur d'une chonique anglo-normandr, a emprunté l'ipisode du meurtre pour sa Jï de Constance, légende appartenant au type de la méchanle belle-mère. Trivet raconte que Constance, pauvre impératrice persécutée, a itẻ recueillic en Northumberland par un chatelain Elda et sa femme Hermingride. In chrralier s̈éprend de la belle inconnue et lui lait des propositions diamous. Repoussé, il se venge un conpant lia gorge à llermingilde, conchée à coté de Constance, en plasant le contean ensanglantẻ sous loreiler de celle-ci et en lacensant ensuite du meurtre. L'aecusateur est epentant frappé jar une main divine et l'imocence de. Constanco est reconnue (v. l'edition de cette rie, accompagnce atune traluetion anglaise, par E. Brock dans les friginals and Inalogues of some of Chawrers Canterbury Fules [18ss].

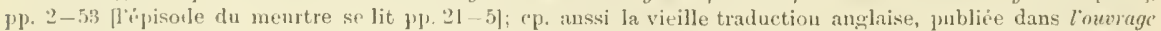

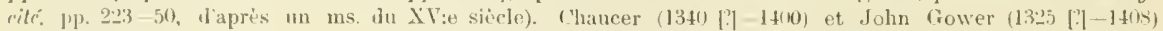
ont siparement remanie le conte de T'rivet, celui-lit dans The Tale of the Man of Lane, celui-ei dans sa r'mufissio Amantis, livre 11 (ponr l"épisode du meurtre dans Chancer, v. Skeat, The Complele Works of freoffrey

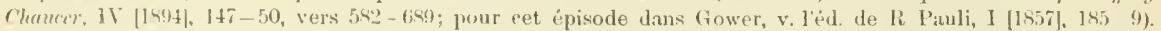

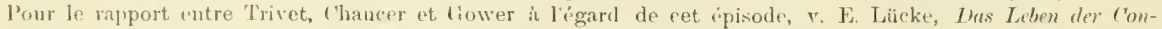

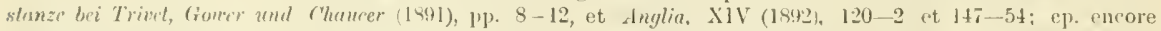

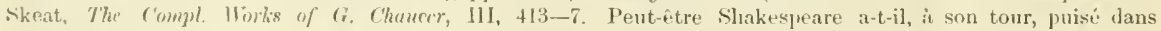

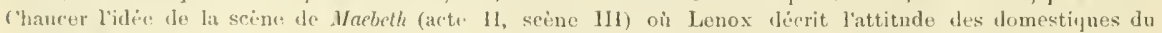
roi assassine:

nThose of his chamber, as it seem'd, hat done't:

"Their hanis and faces were all badg'i] with blond,

„So were their daggers, which, unwipil, we found

"Upon their pillows:" - - - .

(Cp. The Itays of Hilliam shaksperere, 1803, t. X, j. 132. note 8). 
alexandrins (208 lajsses), rersion encore inédite ${ }^{2}$, mais dont il existe me ancienne traduction en prose espagnole, publiée en 1864 par J. Amador de los Rios dans sa Historia critica de la literatura expañola ${ }^{2}$; 2) un remaniement français, de la première moitié du $\mathrm{X}^{\mathrm{r}}$ siecle, de la version primitive perdue du roman de Florence de Rome, version inédite ${ }^{3}$; 3) une romance anglaise, dı $\mathrm{X} I V^{\mathrm{e}}$ on de la première moitié du XV* siècle, publiée par Joseph Ritson dans ses Ancient Engleish Mctrical Romancë̈s (1802)' et par M. Wilhelm Viëtor sous le titre Le bone Florence of Rome (1S!3:3); et 4) le Dit de Flourence de Romme, du commencement du XIV e siècle, publié par A. Jubinal dans son Nouveun Recucil de Contes, Dits, etc. (1839) ${ }^{6}$. La sumce commune de ces rersions était probablement une chanson française de la seconde moitié du XI siècle, actuellement perdue ${ }^{7}$. En comparant les différentes rersions de la branche de Florence de homes, nons constatons que la version-mère a dû diffèrer an moins par les traits suivants (détails superflus omis) de la rersion primitive (indienne):

Personnages: (1) Esmeré, fils de Philipje, roi de Hongrie, plus tard empereur de Rome; (2) Florence, fille d'Oton, emlereur de Kome; (3) Milon, frère ainé d'Lsmeré; (4) Thierri, seigneur de Châtean-Perdu; (5) Nacaire, chevalier auprès de Thierri; (6) un malfaiteor; (7) Escot. Épisodes: (a) très longne introduction,

2 Nous pnblierons sous pen cette version, d'aprés les trois mss. connus (Bibl. nat., nouv, acq. frans. 4192: ms. appartenant it Il. Y.-T. d'Arey Hutton, Marske Hall, Richmond. Yorkshire; Mus, Brit, Landsd. $36^{\circ}$, fragment), dans les publications de la société des anciens textes français.

2 T. V, pp. 391-468; une analyse détallée de cette version se lit ibid. t. V, pp. 6\$ $\ldots 7 t$.

3 Nous publierons également cette version française, d’après le ms. Bibl. nat., f. fr. 2438t, lans notre edition de Florence de firme. Pour la clate, le terminus a quo. l'année 131\%, est fourni par la mention, rers 31. de Fizonne comme type de beauté féminine: car cu personnage est emprunté aux Fưu du Puon, poème 'qui ne peut ètre antérieur à cette date (v. P. Meyer dans le bull. de la Soc. des ane. textes firangais, VIII.

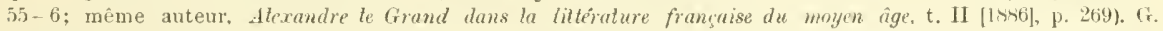
Paris (Rom., XXIII, 81, note 2) a démontré qu'il y a eu leux rédactious du poème intitulé Les limur du F́an, l'une composíe peu avant, l'autre pen après l'anuée 1313. Cp. aussi F. Bonuardot, Rom., XXIV, 5i681. - Pour le teminas al quem, il n’y a d'autre critérium que la langne du remaniement ( $\nabla$. notre édition). + T. III, Pp. 1-92.

' Cette version est conservée par un seul ms.: Cambrilge, [7niv. Libr., Ff. Il. 39.

- T. I, pp. 88-11\%. Pour la date du Thit, v. P. Paris dans l'Hist. litt, de le France, XXV1 (1873),

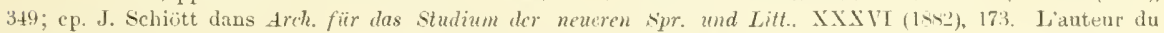
Lit était peut-être Jehan de Saint-Quentin; v. G. Gröber, Grundr. der roman. Phil., II, partie 1 (19(12), p. 910 (\$ $2-23$ ).

7 Nous étudierons cette question de plus prés dans l'Introduction a notre édition de Florenre de Rome.

8 Dans son ourrage intitule frip Fassungen der sage von Florence de lome (1s!90), M. R. Wenzel donne une comparaison analytique très détaillée de toutes ces versions de Florche de Rome. - Ajoutons iei qu'il est possible qu“il ait aussi existé un remaniement en prose de la chanson de Florence de home. puisipue Barbazan, dans le catalogue qu'il avait dressé des manuserits de M. de Bombarde (v. ms. Paris, Bibl. nat., nouv, acr. fr. 1790, fol. 69--70), mentionne comme le '2.):e ms. de cette collection un „Fraginent de Florence de Rome et d'Esmerez. Roman en prose. 14:e siécle"; cp., sur les mss. de M. de Bombarde et sur sa personne, H. Nartin, Cat. des mss. de la Bibl, de l'Ars., VIII (1899), 25:--7. Ce fragment est actuellement introuvable. Il se pourrait cependant que Barbazan se fût trompé en parlant d'un roman en prose. " puisqu'il dit $\left(\mathrm{ms}\right.$. cité, fol. $\left.7 \mathrm{v}^{\circ}\right)$, en indiquant le contenu d'un ms. nacruis par II. l'Abbé de Fleury au mois de fevrier 1752," lequel est actuellement à la Bibl. nat. de Paris (f. fr. 24432), qu'il connaissait "un fragment chez MI. de Bombarde ${ }^{4}$ du "Roman de Flourence de Rome" donné par le ms. en question (fol. 215). Or, ce dernier "roman" n'est autre que le Dit de Flourence de liome, publié par A. Jubinal. Le Iragment de la collection Bombarde a done pu etre tout simplement une partie de ce Dit. 
dans lajuelle il est daconté commont bsmere de llongrie, ajrés la mort d'Oton de Rome et après aroir vailamment combattu te roi de Cirece, ‘ui avait commencé une guerre contre Rome, parco qu'il n'avait pas olitum Florence pour femme, épouse celle-ci et devient empereur; pendant une absence d'Esmeré, occasionnée par la guerre, Milon veut épouser l'lorence, prétendant que son fréro est mort; il est cependant convainea de trahison ot jeté dans une tour; a la nouvelle du retour d'Esmeré, Florence va à sa rencontre, accompagnie te Milon, quille a reläché, et d'une nombrense suite; Milon réussit à séparer Florence de sa suite et la conduit loin de Rome, afin de la prosseiler; sa vertu est cepenlant sauvegardèe par une broche magifue; furicux, Milon l'attache ì un arbre et la bat; à ce monent, il est surpris par Thierri, qui chassait daus la forèt, et prenu la fuite; (b) lenfant de Thiurri et de sa femme Églentine, jeune fille du nom de Biatrice, couche dans lo mème lit que Florence; Maraire jlace le couteau ensanglanté dans la main de Florence dormante; celle-ci va être brûlé, mais est graciée au dernier moment, et on lui rend sa mule et ses riches habits; (c) le malfaiteur devient le sorviteur de Florence et ne tombe pas amoureux ilelle; (f) Esmeré est aussi frarmi les malades; ils arrivent tous séparément au couvent (Beau-Repaire), Macaire accompagné, non soulement de Thicrri, mais aussi de sa femme; (gr) les criminels sont brûlés vifs.

Cette version de Florence de Rome est représentée par deux sous-rersions, l'une domnie par le remaniement français du XIV siècle, l'autre par les autres rédactions '. Si nous examinons d'abord le remaniement français, 'ul, malgré sa date relativement révente, paraît avoir conservé certains détails de la version-mère de Florence de fiome qui ont été altérés dans l'autre branche², nous royons qu'il se distingue par les traits suivants: ${ }^{3}$

Personnages: (6) Gombaut 4 Épisoles: (a) Esmeré et Florence ne sont quue fiancés; (c) Gombaut est en prison chez Thierry; (d) Escot périt dans le naufrage; (f) Thierry est aussi parmi les malades; (g) le mariage d'Esmeré et de Florence s'accomplit à Rome.

2. La filiation des versions peut ètre figurèe comme suit:

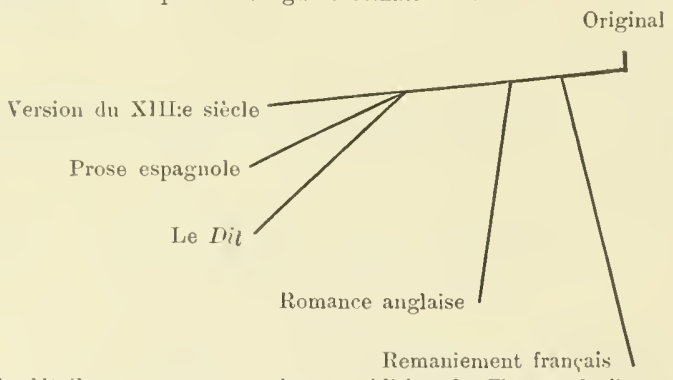

I'our plus de détails nous renvoyous à notre édition de Florence de Rome.

${ }^{2}$ Ces dítails seront examinés dans notre édition de Florence de liome.

${ }^{3}$ Une analyse detaillee de cette version fut domnie par P. Paris, Hisl. litt. de la france, XXVI (1573), 335-50. - Nentionnons en jassant que dans le roman darentures Florent 'l Oclaxim, aujuel cette redaction de Florence de liome fait suite, on raconte longnement les aventures variées doton jusqüa son avènement au tróne. Oton itait fils de Florent et de Marsebille, fille du sondan de Babylone; il était commo jeune appelé Othonet et érousa à la fin l'olice, fille du roi de Palerne. T. l'analyse de P. Paris, Hist. litt. de la France, XXVI (1873), 303-35.

- Nom de traitre; v. E. Samerland, Ganelon und sein Geschlechl im afr. Epos (1596), p. 26 (Gaydon. v. 7889 et suiv.). V. aussi le rỏle do Goubaut de Lausanne dans Charles le Chauve (c). Wenzel, Die Fassungen, etc., p. 52, note). Dans Aiol, „fionbaut" est le nom d'un brigand (éd. Normand-Raynaud [18j7], rers 6665 et 6685). 
Une comparaison arec la version primitive (indienne) montre que dans tous ces cas, excepté pent-être pour le nom du larron, le remaniement français a altéri la version primitive de Florence de Rome.

Quant à l'autre branche, le plus complètement représentée par la chanson française du premier quart dı XII" siècle, elle présente les traits particuliers snivants:

Prsonnages: (6) Clarembaut. Épisoles: (a) relache de Ia tour, Milon va d'abori seul à la rencontre d'Esmeré et accuse Florence d'adultère; convaincu de mensonge, il est chassé par son lrère, mais retourne ¿ Rome et persuade a Florence d'aller à la rencontre du mari; dans la forèt, des bites sanvages yui attajuent Milon contribueut à saurer Florence; (c) Clarembaut est aidé dans sa trahison par un certain Peraut, chez qui il a logé Florence?

Il est assez malaisé de dire si ces traits sont primitifs pour la branclue ou non. Ce qui parle cependant en faveur an moins de l'épisode oì Milon va senl à la rencontre l'Esmeré et accuse l'impératrice d'adnltère, c'est que, grâce à cette acusation dimentie, il est facile de comprendre pourquoi Milon ne retourne pas anprès de son frère, après avoir abandonné Florence dans la forêt.

De cette version française du premier quart dı XIII ${ }^{\circ}$ siècle dépendent directement la traduction en prose espagnole, qui est étroitement apparentée ì l'un des mss. qui la donnent ${ }^{2}$, et le Dit du XIVe siècle. Ce Dit a altéré la version de la chanson française de la manière suivante:

Personnags: (6) Archembatu; (7) Cadot. F́pisoles: (a) l'épisode où Milon accuse Florence d'aduitère manques; il n'est pas question de la broche miraculeuse"; (b) lauteur ne dit pas le nom de la demeure du chevalier Thierry, ni ceux de sa femme et de sa fille; (i) le complice d'Archembaut, dont lo nom n'est pas dit, figure parmi les malades.

Cette version, fortement abrigée et partiellement défectneuse dans l'édition de Jubinal ${ }^{5}$, est empremute d'un caractère très religrienx; ainsi, Florence est, selon l'antenr, accablée de tous ces mallueurs, parce que, en épousant Esmeré, elle rompt un vou de chasteté qu'elle arait fait. La version du Dit ne parait pas être tirée de l'un des mss. existants de la chanson de Florence de Rome ${ }^{6}$, elle provient donc d"un mannserit perdu.

1 hans le roman français, la fin de la chanson, yui n'est donnce que par l'un des mss. (ms. d'Ircy Hutton), est altérée: les criminels n'y sont pas brûlés, mais retonrnent guéris chez cux.

${ }^{2}$ Ms. Bibl. nat, nouv. acq. franȩ. 4192 ; v. notre élition de Florence de Rome.

3 C'est une coincidence fortuite avec la version du remaniement; le lit abrège beaucoup.

* Ce trait noimplique mullement que le Dit ait eu une autre source, conme le veut Mussatia

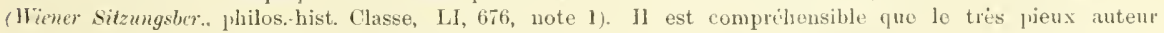
du Thit nait pas voulu attribuer le salut de Florence à un objet de nature mugique.

s Par suite du manque d’un feuillet dans le ms. d’oì l'éditeur a tiré sa version, l'épisode du capjtaine et larrivie de Florence à Beau-Pepaire manquent.

* Comme le us. de Londres n'est actuellement qu'un fragment sans valeur pour la question de la provenance du Thit, il est impossible d'affirmer d'une manière absolue qu'il n'ait pas éte, dans son état primitif, la source du Thit. Le ms, d'Arey Hutton ne peut pas l'ètre, parce que les criminels n'y sont pas brûlés ( $v$. ci-dessus note 1). Quant au ins. de Paris, il ne conticnt pas certaine scène (celle où Milon est attanué dans Ia forêt par un inorme serpent) qui se trouve dans le Dit et yui nous semble être primitive (l'argument le M. Wenzel, Die Fassungen ete, p. 46, en faveur de létroite f'arenté du Fit et du ms. d'Arcy Hutton n'est done pas foudé). 
lieste la romance amyluise, rersion tris libre de lat chanson perdue du XII" siecle. Sille ne diffiere cependant guere, pour les traits yoi nous occupent, de la chanson du XIII ${ }^{\circ}$ siecle, avec laquelle elle forme groupe contre le remaniement du XIV ${ }^{\mathrm{e}}$

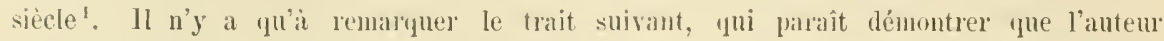
était un homme qui n'aimait pas le fantastigue:

Episode (a): Lanteur no mentionne ni hites sauvapes ni broche miraculeuse: re sont uniqnuenent les priéres de Florence qui otent is Milon tonte sa fore? 2 .

En comparaut les versions prinitives des Ciestu homanorm et de Florence de Rome, on voit que leur soure commune, version ocidentale ancienne, avait altéré la version orientale primitive par les traits suivants:

Personnayes: (1) nn empereur de liome; (4) un grand seigneur; (5) un chevalier, an service du seigneur; (b) un malfiteur. Épisodes: (a) l'héröne fait jeter le bean-frère en prison; i la aouvelle du retour du mari, elle ra ì sa rencontre asee le beaufrere et une nombreuse suite; en route, l'hiroüne et le beau-fréro sont sijaris de la suite; le beau-frere veut abuser delle, mais ne jrarrient pas i ses fins; il l'abaulonne daus la forêt, attachée ì un arbre, et retourne auprés du mari, à qui il raconte quón lui a enlevé sa bellesirur; (b) l'enfant qu'on conlie aux soins de l'hroune est une jeune fille; elles dorment dans le méme lit; l'assa-sin place le couteau ensanglante dans la main de l'héroïne dormante; elle ne reçoit pas d'argent le voyage; (c) le jenne homme raclseté devient le serviteur de l'héroine et ne tombe pas amoureux d'elle; cest jar eupjdité qu'il la vend au capitaine; (f) les quatre criminels ue se reneontrent quau convent.

\section{CHAPI'TRE VI \\ Le Mirucle de la Vierge}

Jusqu'a présent nous n'avons pas vu dintervention divine directe dans le récit de notre héroïne. Elle a bien obtenu, par ses priçes, ce qu’elle a demandé; mais il n'a pas été question d’apparition miraculeuse de quelque être divin dans le lessein le secomir la femme persétutée. Or, il est facile de comprendre que le moyen îge, si porté à erone anx miracles, ait tronvé moyen de faire de notre conte oriental un miracle à la glorification de la sainte Vierge. Le trait miraculeux, cest l'épisorle où la Vierge indique à l゙léroine, endormie sur un rocher an milien te la mer, le moyen de gutérir les lépreux ${ }^{3}$. Un antre trait caractéristique dans ce groupe de rersions, e’est

1 V. p. 30, note 1.

2 Les noms propres de la romance sont naturellement un peu altérís.

3 Ces sortes de miracles ne sont pas rares lans les licgendiaires lu moyen âge. Nons avons aussi retrouve un trait analogue dans une licende loeale moderne de la Sonabe, Legende ron Mariä Florklurg (s.

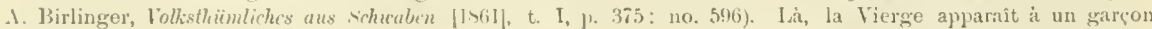
malate et lui dit de rhercher une herbe à un endroit indiqui. Aprés plusieurs exhortations le garçon obeit, et la sainte V̈̈erge elle-meme le guérit en pressaut la planto contre son front. 
qu'il n'y a jamais plus de deux criminels, le beau-frère et l'assassin. l'épisode du capitaine survivant tontetois dans un royage en mer, abontissant à l'exposition de l'héloüne sur un rocher. Comme dans ces versions le bean-fière accuse l'horome diadultire, 'e qui est un trait primitif qui ne se tronvait fras dans la somce commme les Gesta Romanorum et de Florence de Rome (v. ci-dessus p. 32), il est très vraisemblable. que le Miracle de lu Vierge, qui apparaît déjà dans $n$ ms. dlu XIIe siècle, remonte à me version occilentale antérieure à la sonre conmune tes Gesta liomunomm et de Florence de Rome. Cette supposition est corroborée par le fait que, dans le Hiracle de lu Vierge, l'enfant assassiné est, comme dans les versions orientales, un petit garçon, tandis que, clans les versions des Gesta Romanomm et de Florence de Rome, c'est une jemne fille.

La forme la plus ancienne sons launelle se retrouve la version du Miracle de la Trierge est celle conservée dans uń rertain nombre de mss. dn XII siècle et des siccles suivants, contenant en latin des minacles anonymes à la glorification de la sainte Vierge. Dans ses Studien zu den mittelalterlichen Marienlegenden ${ }^{1}$, Ad. Mussafia a donné des indications nécienses sur l'apparition le notre légende (Kaiserin von Rom) dans ces collections de miracles ${ }^{2}$. A l'aide de ces indications, nous arons pu constatel' qne notre Miracle de la Vierge s'y rencontre sous quatre formes différentes, apparentées de très près. Voici par quels traits notre Miracle de la Vierge, tel qüil est domué far ces quatre versions latines, diffère de la version orientale primitive:

Personnages: (1) un empereur romain; (4) un noble seigneur; (5) Je jeune frère du seiguenr; (6) manque; (7) des marins. F́pisods: (a) l'empereur, qui part pour la Terre Sainte, laisse l'empire et son jeune

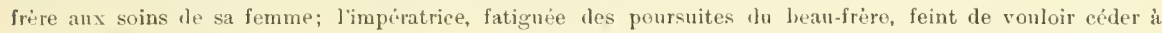
ses prières; elle fait donc mettre en ordre une tour spéfiale pour leurs rendez-vons, mais, anssitôt que le beau-frère y est entré, elle l'y enferme et l'y tient prisonnier eing ans ${ }^{3}$; i la nouvelle du retour de l'emperour, l'imprératrice, pleine de joie, fait mettre le bean-frèe en liberté; celui-ci sf hâte d'aller ì la rencontre de son frère et, questionuc sur sa figure pâlie, raconte que l'impératrice avait voulu le séduire et 'pue, pour se sonstraire à ses tentatives, il s'était tenu enfermi lans une tour; l'empereur, saisi de clagrin, s'évanenit, puis continue sa route vers lome; quand limpératrice vient à sa renrontre, il la fraple au visage et dome ¿ deux valets l'orlie de la concluire dans une forét pour l'y derapiter; au moment où les valet, veulent la violer asant de la tuer, ils sont surpris et mis ì mort jar le noble seigreur et sa suite; (b) l'assassin place le couteau ensanglantí dans la main de l'impératriee, domant à côté du petit lils du seigneur; par ses aris d'eforoi, l'imperatrice éveille le seigneur et sa femme; flle est conduite ì la mer pour être transportée dans un autre pays; l'épisole (c) mauıne; (d) sur le navise, les marins veuleut abuser d'elle, mais, émus par ses lritres, ils se contentent de la déposer sur un roclser désert, an milien de la mer; lia, la sainte Vierge lui

1 V. Silzungsherishte der philos.hist. Ciasse der Kais. Aliad. der Wissensthaflen, Vienne, t. CXIII (1ssi), pp. 917-94 (= I); t. CXV (1888), pp. 5-92 (- II); t. CXIX (1889), wém. 1X, pp. I-66 (- III); t. (XXX1X (

${ }^{2}$ Cp. aussi Ad. Mussafia, Über die non Gautier de Coincy benülzten quellen (dans les Denksehriftn der

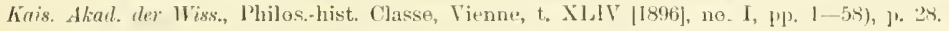

3 Comme la branche de Florence de lome parle aussi d'une low (v. (i-dessus j). 30), il est jussible yue co trait soit jopres a la branche occidentales primitive. 
inclique, dans un songe, une herbe, croissant sous sa the, avec laquelle elle pourra gucirir les lépreux'; l'impriatrice eneille de cette herbe, et peu ajrès un navire lin recueille et la conduit a la terre ferme; (e) sortie Ju navire, l'impératrice guirit un lijreux et ensuite beacoup d'autres lépreux; il nest pas question de couvent; $(\mathbf{f}-\boldsymbol{q})$ l'ussissin, devenu lipreux et ayant entendu parler de ces cures merveilleuses, fait appeler la femme; il ne confesse son crime įuajpès uno seconde sommation; on reut que limpératrice éfouse le jente homme guiri, mais elle rufuse et continue son chemin vers Rome; le frere de l'empereur itant devemin lineux, l'impiratrice est apuelíe aujres de lui et le gurit en la présence de son mari, du jaje et du sinat; apris settre fait comnaitre, elle entre dans un couvent.

11 fatit probablement regarder comme la plus ancienne de ces yuatre versions latines relle, la senle donnée par un ms. du Xll" siècle"2, qui commence par les mrots: r(wam mercelem legale conitzgitum caste servantes obtincant in futmro, nondum, fratres carissimi, rognoscimus" , et qui, dans les mss., apparaît sous les rubrigues: De pudicitia ct tolerantia miustam imperatricis ${ }^{3}$, lye improtrice quam ex omni bribulatione salvucit (sc. Virgo Beata) et Exemplum de quadam imperatrices. Nous désignerons cette version comme le Miracle latin A.

Lal seconte version du miracle, que nons appellerons le Miracle latin $B^{6}$, commence far les mots: "Fuit quidam Rominus imperator, cui providit Dei clementia uxorem genere ot moribus nobilissimam ac ptrldnitudine honestissimam", et est divisée en trois clapitres portant les rubriques: 1. De quadam imperutrice cuius castitatem beata virgo a violentia servorum eripuit; 11. Ie alio casu consimili circa candem imperutricem; 111. De medicina quam beatu viryo imnotuit. Cette version n'est quin remaniement raccourci, mais fidèle, dı Miracle lutin $A$. C’est elle que le cèlèbre historiographe Vincent de Beauvais (mort en 1:264) introduisit mot potur mot dans son Spreculum Historiule, dont nous parlerons plts tard ${ }^{7}$.

II ne s'acyit plus pue de ectte maladie; dans les trois branches précédentes les maladies variaient.

2 IIs, Paris, Bibl. nat., f. lat. 146,3 (fol. $36 \mathrm{r}^{0} \mathrm{a}$ ). Les autres mss. qui, it notre conuaissance, con-

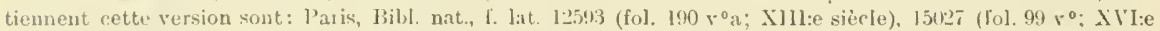
siucle), 1605i (fol. $25 \mathrm{r}^{\circ} \mathrm{a}$; Xlll:e siecle) et 18134 (fol $126 \mathrm{r}^{\circ} \mathrm{a}$; XlII:e siccle), ainsi que le ms. Paris, Ars. 946 (fol. $27 \mathrm{r}^{\circ} \mathrm{b}$, sans rubrique, XIIl:e s.), et trés probablement le ms. Charlerille los Xlll:e siècle; indiqué par Mussntia, oum cite t. CXY, 1, 50), que nous navons pas en l'ocrasion de consulter, mais qui, d'après Mussalia, commence jar une phrase (n Erat liome seculari habitu imperator honorabilis, ficle et religione venerabilis, verbo et artu in regno amabilis") qui se retrouve presq̨ue mot pour mot dans le texte des autres mss. apris la petite introduction. (Juant au ms. aljpelí par Mussafia Ps, nons n'avons pas réussi a l'ilentifirr; aplarenti te trés prés au ms. Paris, Bibl. nat., f. lat. 16056 (v. Mussafia, ouvr. rite, t. CXIll, lp). (59) - 60 , i] doit cependant contenir la méme version. - Nous donnons la version du ms. Bibl. nat., f. lat. $1+46.3$ dans notre Appindipe (1). Les versions des ciny antres mss. parisiens ne diffirent que jour des ditails insigniliants de la version du ms. 13ibl. nat, f. lat. $14+63$.

3 .lss. Paris, J'3ibl. nat., I. lat. 11463, 15027 ct 16056, ansi que trís probablement celui disigne par Mluscalia founr, eite, t. ('XIH, p. 459) par lo sigle l's, leguel nous navons pas reussi a identifier.

+ Ms. Paric, Bibl. nat., f. lat. 12593.

- Mls. Paris, Bibl. nat., f. lat. Isti3t.

- Nous l'avons trouvie dans le ms. Paris, Bibl. nat., f lat, nour. acı. 357 (fol. 1; XlJise sièrle).

7 Nour devons ceprendint avoner 'qu'il nous semble tont au moins possible que le Mir. lat. I soit unu simple copie du ricit du Vincent de Beauvais. Celui-ci anat dans ce cas remanie la version alu Mir lat. A 
La troisième rersion, le Miracle latin $C$, est apparentéc de très près à la version $\mathrm{B}$ et commence à peu près yar les mêmes mots. Nous ne la connaissons que d'après un ms. dı XIII ${ }^{\mathrm{e}}$ siècle $^{1}$.

Enfin, la quatrième rersion, le Firacle latin $D$, est nu remanicment en vers, composé de 8.2 quatrains de vers décasyllabes, lequel, pour le fond dn récit, suit de près les trois antres versions ${ }^{2}$. Cette version porte le titre: De imperatrice miraculum, et commence par le couplet suivant:

„Fuit olim vir quidam nobilis,

Urbis Rome dux venerabilis,

Fide constans et facto stabilis,

Juxta causam judex immobilis"s.

Comme il a déjà été dit plus haut (p. 34), la version A du mirucle latin se rencontre dans un ms. dn XII" siècle, mais il est possible que le miracle en question remonte jusqu'au siècle précédent, car cest à partir du milieu du XI ${ }^{\text {e }}$ siècle que ces sortes de miracles deviennent communest. Qnoi qu’il en soit de l'époque précise de la composition de ce miracle, ce n'est qu'an XIII ${ }^{\mathrm{e}}$ siècle qüil a en nn déreloppement remaruquable. grâce surtont à deux auteurs bien connus, le moine français Gantier de Coinci $(1177-1236)$ et le célèbre historiographe Vincent de Beauvais (1190-1264). Mais avant de parler d'eux et de leur rôle dans l'histoire de notre légende, il convient de mentionner à cet endroit l'apparition de notre miracle dans quelques recueils anonymes de miracles de la Vierge, écrits en français, en hollandais et en islandais, qui sont des traductions de recneils de miracles latins.

En français le Miracle de la Tierge se rencontre en trois versions différentes, dont deux en prose et une en vers. Linne des versions en prose, que nous arons trouvée dans denx mss. dn $X V^{\mathrm{e}}$ siècle $^{5}$, porte la rubrique: De Nostre Dame, qui yarda la femme de lempereur de plusieurs perilx. Elle ne diffère guère dn minacle latin. Les divergences les plus remarquables sont:

Ms. Londres, Brit. Mus. Harl. 2316 (fol. $6 \mathrm{r}^{\circ}-7 \mathrm{v}^{0}:$ no. 12), Nous donuons cette version, jusqu'ici inédite, dans notre Appendice (E).

${ }^{2}$ Mentionnons cependant que, dans la version $I$, le beau-frère dit ì l'empereur que c'est l'impératrice ‘ui l'a enferné dans la tonx, et qu’il n'y est pas dit ‘ute l'empereur frappe sa femme.

${ }^{3}$ Nous avons trouvé cette version, daprès les indications de Mussafia (Sitz. ber., t. CXIII, pp. 979 et 981), dans les mss. Paris, Bibl nat, f, lat 17491 (fol. 155 rº; XIII:e siécle) et 2333 A (fol. 119 ro; XIV:e siécle). Nous donnons cette version inédite dans notre Appendice (F) d'après le premier des mss. cités. L'autre ne présente en général que des variantes insignifiantes.

+ Cp. Mussafia, Wiener sitz. ber, philos.-kist. Classe, t. CXIX, p. 57. - Ces collections de miracles de la Vierge étaient connues sous les noms de Marionate ou de Jariale. Le jremier nom se rencontre dans une version hollandaise de notre miracle dont nous parlerons ci-dessous, le second nom (nariale magnum ${ }^{4}$ ) se tronve dans Vincent de Beauvais.

${ }^{5}$ Paris. Bibl. nat, f. fr. 1805 (fol. $90 \mathrm{r}^{0}-95 \mathrm{r}^{0}$ ) et 1506 (fol. $61 \mathrm{r}^{0}-6+\mathrm{v}^{0}$ ). Nous clomnons cette version, daprès le premier des mss, dans notre Appendicc (G). 
Fy isodes: (a) l'empereur confie sa femme à son frère; le beall-frére raconte à l'empereur yue limpératrice l'avait enferme dans une tour', jarce qu'il avait voulu s'bloigner, exaspéré de la vie scandaleuse que menait sa belle-steur.

Lautre version françise on prose se tronve lans mu ms. de la Bibl. nat. de Paris ${ }^{2}$ qui porte le titre: Faiz ct miractes de Nostre Dame ${ }^{3}$. Notre miracle s'y lit sous la rubrigue: De lu frmm d'un cmpereur qui pour bien fere eut a souffir moult de maulx, et estoit en grant perill de morl, laquelle Nostre Iname delivra pour ce. que elle se reclama a elle. Pour le contenn, cette version ne diffère non plus du miracle latin que par des détails insignifiants':

Épisodes: (a l'empereur, qui part pour des contries doignées de son empire, confie l'empire et sa femme aux soins du frire; ce sont quatre ,escuyers" qui recoivent lordre de tuer limpiratrice; (b) l'inıp'ratrice est juacie. sans vivres, dans un petit bateau; (d) éest de cette situation que la tirent les marins qui veulent ensuite la riolenter.

Ia version francaise en vers, entin, qui ne nous est comnue que prar un manuscrit de la Bibliothèune de l'Arsenal de Paris ${ }^{5}$, commence palu les rers:

,1)ames, qui vostre mariage

Tenés en loi de pucelage." - -

et porte la rubrique: Ine la sainte mpereris qui garissoit les lieprous. Cette version est, pour le fond du récit, tout à fait identique au miracle latin.

(Quant à la version hollandaise, nous ne la comnaissons que par mu onvrage imprimé à Leyde en 150:3 par Hngo Jan Soen van Woerden et portant le titre: Die miraculen van onse lieue vrour.. Notre minale y est intitulé: Fan eenre keiserinnen et commence ainsi: „En leest in een boeck van onser liener rrouwen exempelen dat glelieten is murionale. Dat te romen was een keiser lye hadde een goede eerbaer rronwe seer innich tot gode", etc. ${ }^{6}$ Cette version ne diffère du miracle latin que pour чuelınes détails:

Épisodes: (a) le beau-frère raronte il son frère que c'est l'impératrice qui l'a fait eufermer dans la tour; (d) les marins jettent limpératrice dans la mer sur une flanche, et elle est ainsi conduite par les flots it lîle.

En islandais, notre miracle se rencontre dans trois rédactions différentes, qui ont ité publiées, en 1871, par C. R. Unger dans son onvrage intitulé Muriu

1 ('e trait, quion a vu déjà dans Ie Miracle latin $D$, pourrait bien être primitif; car il est peu naturel que le beau-frère explique sa pâleur par un séjour volontaire dans la tour. On le reneontre, d'ailleurs, dans plusieurs autres versions, dont nous parlerons plus bas.

${ }^{2} \mathrm{~F}$. fr. 410 (XT:e siecle), fol. $15 \mathrm{r}^{\circ}$.

"A la fin du volume on lit: "Explicit per dei gratiam liber miraculorum virginis marie".

- Nous jublions aussi cette version française en prose dans notre dppendice $(\mathrm{H})$.

${ }_{5}$ No. 35115 (XI1l:e siècle), fol. $133 \mathrm{r}^{0} \mathrm{a}-136 \mathrm{r}^{0} \mathrm{a}$. Une miniature qui se trouvait avant la rubrique au bas du fol. $132 \mathrm{y}^{\circ} \mathrm{b}$ ) a éti enlev'e. - Nous donnons cette version dans notre Appendice (I).

- V. fol. p II $\mathrm{r}^{0}$. Le miracle va jusquau fol. p IIII $\mathrm{v}^{0}$. - Nous avons utílisé un exemplaire qui se trouvo an Musie Pritannique. 
Saga ${ }^{1}$. Pour le contenu, ces trois rédactions, dont l'une est incomplète an commencement $^{2}$, ne diffèrent guère du miracle latin. L'une des rédactions, intitulée Wor frv frelsadi drottningu (Notre Dame saura la reine) ${ }^{3}$, se rapproche beancoul dans sa forme du Miracle lutin $A$. Les deux autres, dont celle qui est complète est intitulée $A f$ keisara fru (De la femme de l'empereur). lappellent plutôt les Miracles latins $B$ et. $C$. Il faut seulement mentionner que la rédaction intitulée Af keisara fru présente un certain nombre d'amplifications de détail.

Le miracle, tel qu'il a été raconté, vers $1218-1222^{4}$, en vers français par Gautier de Coinci dans ses Niracles de Notre-Dame, a été publié par M. Méon, en 1823, dans son Nouveau recueil de fabliaux et contes inélits ${ }^{5}$. Le miracle y porte le titre: De l'Empereri qui gardu sa chastée par moult temptacions, ou de l'Anpereriz de Rome qui fu chacie de Rome pour son serorge, et commence ainsi:

"As saiges dit et fet savoir

Li très bons livres de savoir"

1 V. pp. $421-38,1104-12$ et $1112-6$.

2 Cest la rédaction imprimée en dernier lieu. Ello commence au moment oủ le seigneur qui a sauvé l'imperatrice des mains de ses bourreaux la conduit chez lui.

${ }^{3}$ C'est celle donnée pp. 1104-1'.

+ V., pour cette date, les Miracles de la Sainte Vicrge, éd. Abbé Poquet (1857), p. XXXVIII. Dans le Grundriss der romanisehen Phitologie, t. II, partie I (190-), p. 651 (\$ 99), M. G. (iröber place la composition des Miracles de Nostre Lame vers 1223.

s V. t. 1I, Pp. 1-128 (4064 vers plats de huit syllabes; le récit finit dejjà avec le vers 3983). La citation erronée "II, 50 et suir.", qui se rencontre sonvent la première fois dans Monmerque-Michel, Théâtre Francais, 1839, p. 365), provient d'une citation mal comprise de Fr. Michel dans son ćdition du foman de he Violette (1S34), p. 192, note 2. Michel roulait dire que l'épisode du meurtre commence, dans Méon, p. 50, ce qui est juste.

- Méon nindique pas la source de sa version; il est probable qu'il a utilisé plusieurs mss., ce qu“indique déjà le double titre; cp. A. Weber, Handschriftl. situdien auf dem Gebiete romanischer Literatur des Mittetatters (1876), p. 6. Dans le ms. Paris, Bibl. nat., f. fr. 23111 (XIIT:e s.), notre miracle (fol. $253 \mathrm{v}^{\circ}$ b) porte le même titre que la première partie de la rubrique domnée par Méon; cp. A. Weber, ouvr. eité, p. '27. D'autre part, la seconde partie de la rubrique est donnée par le ms. Paris, Bibl. nat., f. fr. 24300 (XII:0 s.; fol, 83 r. ${ }^{\circ} \mathrm{b}$ ). Les rubriques du miracle étant très variées dans les différents mss, nous en domnons ici un petit échantillon:

De l'empereris de Romme qui garda chasteté en moult de temptacions (ms. de Soissons, miracle no. 4); v. l'Abbé Połuet, Les Viractes de la Sainte Vierge, traduits et mis en vers par Gautier de Coiney [185i], p. XXXIII);

De lempeerris qui garda sa chasté contre mout de temptations (ms. Paris, Bib], nat., f. fr. 986 [XIII:e s.], fol. $\left.1113 \mathrm{r}^{\circ} \mathrm{b}\right)$

D'une emper[er]is de Rome qui souffrit moll pour mantenir eastetez (ms. Berne, Steiger-Mai [XV:e s.], fol. 142 $\mathrm{r}^{0} \mathrm{a}$; v. A. Tobler dans Jahrb. für roman. und engl. Lit, VII [1866], 433, et A. Weber, ouvr. cité, pp. 7 et 9 );

I) la bonne enpereris qui garda loiaument sen mariage (uss. Paris, Bibl, nat., f. fr. 25532 [XIII:e s.], fol. $109 \mathrm{r}^{\circ} \mathrm{a}$, et ms. Paris, Ars. 3519 [XVIILe s., partiellement en résumé], p. 62 a);

De l'ampereriz de Rome qui mout aimme Wostro Dame sainte Marie (ms. Lontres, Brit. Mus. Harl. 441 ] [XIV:e s.], fol. $\left.108 \mathrm{r}^{\circ} \mathrm{b}\right)$; 
Cette version ne diffère dn miracle latin par ancun trait important, mais seulement par des amplifications tle détails'. Comme le montre déji le grand nombre de manuscrits qui ont conservé l'ixnve de Giautier de Coinci, ses miracles ont dû jouir d'une très grande notoriété. Il n'est tonc pas étonuant de retrouver son miracle de l'Impératrice de Rome dans la l'éninsule ibérique. Arl. Mnssatia en a publié, en IS6ti, une traduction espagnole en prose du $\mathrm{XIV}^{\top}$. vièrle dans son némoire Eine altspanische Prosadarstellung aler Crescentiasage", traduction indirecte faite d'apris me traduction galicienne perdue $^{3}$. Dans le senl ms. guon commisse de la traduction espagnole ${ }^{i}$, le miracle porte ce titre: Ifuy fermoso cuento de una sunta emperatriz que ovo en Rroma ct de su castidat. Cette traluction est très filc̀le.

Nous avons déjà dit plus laut (1. 34) qu'une des versions du miracle latin amonyme a été introduite par l"historiograple Vincent de Beaurais dans son Speculum

De lempereis de Rome qui cts stronges ama (ms. Chantilly, Musée Condé 1578 [XIII:e-X]V:e s.], fol. 167; v. Chantilly, Le Cabinet des lirres. Monuserits, tome Il (1901), 1). 55 [no. 475]);

The la feme de lempereur de liome quy ful traii du frere de son mari et qui Nostre Dame ayda et la remist a son honneur (ms. I'aris, Bih], nat., f. fr. 1613 [XlII:e s.], fol. 151 vob [la rubrique a été ajoutée postérieurement par une main du XV:e siecle]);

De l'empereriz qui fu tentee en multes tribulacions (ms. Blois 34 [XIIl:e s.], fol. V1 Xx X vo; v. Cat. gén. des mss, des bibliolhèques publiques de France. Déparlements, t. XXIV (1894), p. 392);

De la bone empereriz qui garissoil les mesiaus par miracle (ms. Paris, Bibl. nat., f. fr. 1533 [XIII:e s.], fol. 14 in $\left.\mathrm{r}^{\circ} \mathrm{a}\right)$;

Ite l'empereris de Roume (mss. Paris, Ars. 3517 [Xlll:e s.], fol. $148 \mathrm{r}^{\circ} \mathrm{a}$, et Paris, Bibl. nat., f. fr. 1536 [SIII:e s.], fol. $\left.120 \mathrm{r}^{0} \mathrm{a}\right)$;

The inperatrice que per mullas tenptaciones castitatem seruauit (ms. Paris, Bibl. nat., f. fr. 817 [ann. 1465], fol. $\left.75 \mathrm{v}^{\circ} \mathrm{a}\right)$;

The imperalrice (mss. Paris, Bih] nat., f. fx. 2163 [XI1]:e s.], fol. $103 \mathrm{v}^{0}$ a, et 2193 [XII:e s.], fol. 2l $\mathbf{r}^{0} \mathbf{a}$ ).

Ajoutons enfin yu'il n'y a pas de rubrique dans les mss. suivants: 1) Paris, Bibl. nat., f. fr. 22928 (XIV e s.), fol. 160 $r^{\circ}$ (le commencement du miracle fait défaut); 2) Berne 634 (XII1:e s.), fol. $99 \nabla^{0}$ ( . J. R. Sinner, Catalogus fortieum mss. Bibliothecae Bernensis, t. IIl [1772]. p. 389); cp. H. Hagen, Calalogus codieum Bernensium [Berne 1875], \}. t90 [n. Miracle de l'imperatrice“]); 3) Paris, Bibl. nat., f. fr. 1530 (XIII:e s.), fol. $147 v^{\circ}$ a (les rubriques des autres miracles sont en latin).

1 Dans l'Histoire lilleraire de la Franee, $\mathrm{\Delta IX}$ (1838). \$50-5, A. Dural a donné une analyse de la version de Gautier de Coinci d'aprés le ms. Jaris, Bibl. nat., no. 2710 du Calalogue des livres de la biblio-

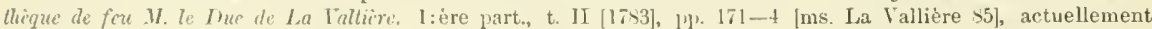

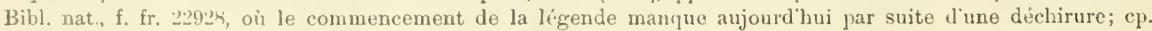
P. Paris, Les mss, francois. VT [18t5], 31s).

${ }^{2}$ V. Silzungsberichte der kais. Akad. der Hisscnschaflen. philos.hist. Classe (Vienne), t. LIII (annéo 1Sfifi), Pl. 499-565. Notre légende y occupe les pp. 518-6i2.

3 Le traducteur castillan dit lui-même: ,desto vos quiero retraer fermosos miraglos, asy como de latin fué tresladado en francés et de francés en gallego" (cp. Mussafia, ouvr. cilé, p. 5(10).

- C'est le mîme ms. (Escurial 1., j. 12), intitulé Flos Sanctorum, qui contient la version espagnole de Flornce de Lome (r. ci-dessus p. 29). - A. Ebert (Jahbuch für romanisehe und englische Literatur, IV [1862], 5!) a le premier signali: la prisene de cette version castillane du miracle de l'Mmpéralrice de Rome dans le ms. en question, indique par lui sous la cote h-I-13. 
historiale, achevé en 1244 on $1254^{1}$. Le miracle y occnpe les chapitres $90-92$ du septième $^{2}$ livre (époque de l'emperenr Tibère) et y porte ces rubriques: Cap. XC: De Imperatrice cuius castitatem /Beata Firgo/ a violentia semorm eripuit; Calp. XCI: De alio casu consimili circa candem Imperatricem; ('al). XCII: De modicina quam ei Beutu Virgo innotuit. L'on connaît beaucoup d'éditions de l'ouvre de Vincent de Beauvais, ainsi que des traductions en néerlandais et en français. La tradnction néerlandaise, qui est en vers, fut exécutée par le poète flamand Jacob van Maerlant. entre les amnées $128+$ et 1290 , sous le titre Spiegel Historiacl, et notre miracle (300 vers) y est appelé: Tan der lieyserinnen van liome ${ }^{3}$. La traduction française, Miroir historial, qui est très fidèle, ne date que du XIV" siècle ${ }^{4}$ et a pour auteur .Jean du (ou: de) Vignay ${ }^{5}$. La version de Vincent de Beanvais de notre miracle a aussi été publiée par H. F. Massmann dans son édition de la Kaiserchronik (1854) ${ }^{6}$; me traluction en italien se trouve dans Fr. S. (Quadrio, Della storia e della ragione d'ogni poesia ${ }^{7}$, et 11. W. A. Clouston donne, dans les Originals and Analogues of some of Chaucer's Canterbury Tales (1885), nue trarhetion en anglais des chapitres XC et XCI".

Dans la première moitié du XIV" siècle, la version de Vincent de Beanvais fut utilisée pour le célèbre recueil d'exemples intitulé Scala celi, qui avait pour auteur le dominicain Johannes Gobii, appelé ordinairement Johannes Junior. Notre miracle, qui

1 V., pour ces dates, l'Hist. litt. de la France, XVIII (1835), 456. - Nous répétons ici ce que nons avons dit déjà p. 34, note 7: quil est possible que le Mir. lat. $B$ ne soit qu une copie le la version de Vincent de Beauvais, qui aurait, par conséquent, utilisé plutôt le Wir. lat. A. Ce qui pourrait être considéré comme une preuve en faveur de cette opinion, cest que le ms. qui contient le Mir. lat. $C$ (apjarenté de très près au Wir. lat. B) renvoie pour plusienrs miracles (cependant pas pour le nôtre) à l'ouvrage de Vincent de Beauvais comme source.

2 Dans les plus anciennes éditions, du moins l'èlition princeps de 1473 (imprimce à Ntrasbourg par Johannes Mentellin) et celle de 1483 (imprimée à Nuremberg jar Antonius Koburger), dans lesquelles on compte un livre de plus au commencement (contenant le prologue et la table des matiéres des trente-deux livies du spec. hist.), c'est le huitième livre.

3 V. l'éd. de M. de Vries et E. Verwijs, l [1863], 3405 (partie I, livre VII, chap. LXV). Dans l'éd. de J. A. Clignett et J. Steenwinkel (Spiegel Historiacl of Rijmkronijk van Jacob van Macrlant, Leyde 1r8t-5), notre miracle se lit t. II, pp. $220-9$.

+ Elle a probablement été faite en 1332-1333; v. L. Delisle Exemplaires royaux et princiers du Miroir Historial (extrait de la Gazelte archéologique, année 1886), pp. 4-6. La premièe juditon imprimée dato de 1495-1496 (Paris, Vérard); cp. J.-Ch. Brmet, Manuel du libraire, t. V (186t), col. 1256.

${ }^{\circ}$ V. l'éd. princeps, t. I, fel. CCLXXXVil (doit ètre: CCX(IX) v ${ }^{\circ} \mathrm{b}-\left(\mathrm{C}(\mathrm{N})\right.$ (doit être: ( $(\mathrm{CI}), \mathrm{r}^{\circ} \mathrm{b}$; l'éd. de 1531, t. I, fol. CCXXI $\mathrm{r}^{0} \mathrm{~b}-1$ 'CXXIl $\mathrm{v}^{0} \mathrm{a}$. Le miracle se trouve dans le huitieme (v. ci-dessus note 2) livre, chapp. 90-2. Les rubriques des chapitres sont, d'après l'èd. de 1495: Xr': lhe lemperiere de laqualle elle [la mere dien] garda la chastete ot defendit quelle ne fut corrompue ne violee de ses manuais serfs; XCI: Dung aulre cas semblable qui aduint a celle emperiere mesmes: XCII: Do la medecine que la benoiste vierge pucelle marie lui demonstra en la roche de la mer done clle fut puis essauciee sur ses ennemys.

- T. III, pp. $899-902$.

7 T. IV (1749), pp. $351-4$.

${ }^{3}$ Pp. 400-1. Lomission du dernier chapitre est probablement due à une erreur commise par plusieurs savants (le premier, à notre connaissance, était Fr. louce, Illustr. of' Shakspcare [180i], 11, t20), qui n’ont cité que les deux premiers chapitres de la version de Vincent de Beauvais. 
y occupe la sixième place sous la rubrique alphabétique Castitas, ne présente avec sit source que quelques dilférences de pure forme ${ }^{\text {. }}$.

Parmi les écrivains qui, de lem propre areu, ont ntilisé la rersion de Vincont de Beanvais, il fant ensuite nommer le dominicain Jolnames Herold $(\div \text { en } 1468)^{*}$, appelé d'ordinaire "Discipulus", qui en tit la premier miracle de son Promptuarium de

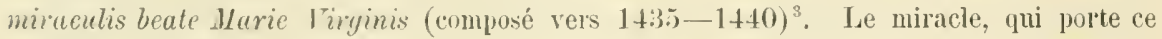
titre: Imperatrici subcenit Maria in maximis angustiis, ne diffère que dans les termes de la version de V'incent de Beauvais; il est un peu abrégé. En 1728, le dominicain Bonaventura Elers réimprima le Discipulus sous ce titre: Discipulus redivivus: seu sermones discipnli quadragesimules et festivales cum Promptuario Exemplorum ${ }^{4}$.

Un autre yui dit également avoir puisé dans Tincent de Beanvais fut lillustre prédicateur italien Gabriel Barcleta (Barleta, Barletta, Bareletta; mort probablement apre's 1450). Sa version du miracle, dans ses Sermones tum quadragesimales quam de sunctis $^{5}$, est en effet tout ì fait conforme à celle cle Vincent de Beaurais.

La version de Vincent de Beauvais a encore, dans un état tout à fait intatt, été introdnite dans le célèbre recneil de légendes pieuses comu sons le nom de Speculum exemplorum, dont la première édition parut en 14\$1. Le miracle porte dans la table ce titre: Castitatis merito imperatrix per Beatam Virginem ex multis angustiis liberatur ${ }^{6}$. Dans la refonte de cet onvage par un anonyme (le jésuite Johannes

1 T. led. de $1+76$ (Juber), fol. XLlV $\mathrm{r}^{\circ}$-XLVII $\mathrm{r}^{\circ}$, oil, par erreur, la rubrique est Caritas. Dans liulition de $1+80\left(\mathrm{I}^{\top} \mathrm{lm}\right)$ il $\mathrm{y}$ a Castitas. Pour d'autres editions, v. T. F. Crane, The Fiempla - of Jacques de Vitry $(1 \times 90)$, p. IXXXXvi, note + .

2 V., pour cette date, Loniventura Elers, Thiseipulus redivinus: seu Sermones Discipuli dominicales, quadragesimales at festivales, eum t'rompluario excmplorum: - - (Augsbourg 172〉; le rolume ne contient que

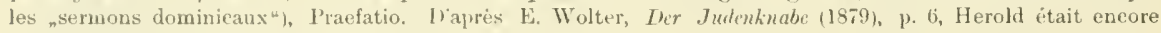
en 1470 cillebre comme pridicateur ì Bale.

${ }^{3}$ Y., pour cette date, T. V. Crane dans les Proreerlings of the Ameriran Plitosophical Soricty. t. XXI (14st), p. 54, mote * et the Errmpla - of Jarques de Fitry (1890), p. LVII. Liouvrage de Herold a souvent iti: imprime aux XV:e et XVh:e siécles. Nous nous sommes servi dume édition sans date, lien ni pagination, prolablement imprimie it keutlingen, en 1475 .

+ Notre miracle se lit 1). 8.51 b s5t a (no. 635). - Dans les Procedings of the Amer. Philos. sioe.. XXI (18st), 5s, note 's, M. 1. F. ('rane dit que louvrage intitulé La fleur des commandemens de Dicu, dont it existe plusieurs inlitions de la tin du XV:e pt de la lière moitié du XVlie sicele (v. J.G. Th. Graesse, Trésor

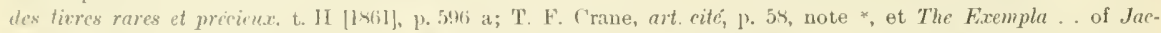
ques de Vitry 14901 1. 'XIII, note "l, est une traduction du Prompluarium de Herold. ('ela est une erreur, conme M. Crane parait lavoir remarıué lui-même jlus tard (v. The Errempla etc., pp. CXIII-CXIV); aussi notre milacle ne sy rencontre-t-il pas (nous avons consulté une édition de 154r, Paris, par Jehan Real).

${ }^{5} \mathrm{~V}$. leed de $1497-1498$ (13rescia, in $4^{\circ}$ ), part. 1 (1497), ff. $38 \mathrm{r}^{\circ} \mathrm{b}-38 \mathrm{r}^{\circ} \mathrm{b}$. Dans l'éd. de 1504 (Lyon, ('I. Havost), le miracle se lit part. I, ff. $1.11 \mathrm{v}^{0} \mathrm{a}-\mathrm{I}$ HIl $\mathrm{r}^{\circ} \mathrm{a}$. Concermant les diverses ciditions de ces sermones. v. 1). ('lement, Bibliolheque curieuse historiquc et critique on Catalogue raisonne de lizres difficiles à trouver, t. II (1751), 11p. 425-7. - Le miracle est place parmi les sermones qualiagcsimules ,in secundo Sabbato quadragesime ${ }^{-}$sous la rubrique ginirale: .'suod Naria est aduocita nostra .

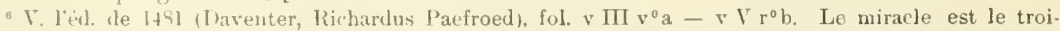
sieme -exemple ${ }^{4}$ de la distinctio quata“. Four les nombreuses iditions de cet ourrage. v. T. F. C'rane dans les Propcedings of the Ameriean Philos. Sinciety. t. XXI (1584), p. 60, note*, et The Eximpla - - of Jacques de Vitry (1s:1), 1, LXXIV, note $\neq$. 
Major, né en 1542, mort en 1608), laquelle porte le titre: Magmum Speculum Exemplomum, on lit à la tête de notre mirarle: Beata virgo Maria Imperatricem castissimam, murito fidelissimam, \& propler corporalem pulchritudinem multu porpessom a tribulationibus liberat ${ }^{1}$.

Mentionmons encore que le Père Don Silvano Razzi, frìre ainć de l'illustre dominicain florentin dn $\mathrm{XVI}^{\mathrm{e}}$ siècle, Seraphino Razzi ${ }^{2}$, introduisit la version de Vincent de Beaurais dans son ourrage en italien: Miracoli della gloriosa T'pryine Muria nostru. Signora, ò elle est le premier miracle dn second lirre: Maria somuine ad ona Imperatrice posta in granissime anyustie ${ }^{3}$.

Enfin. le célèbre écrivain religieux allemand Martinus von Cochem ( $\leftarrow$ en 1712) raconte le miracle, d'après Vincent de Beauvais, dans son Anszerlesenes History-Buch ${ }^{4}$. Le récit, qui porte le titre: Wie die Mutter Gottes einer Keyserin in ihrrn grossen Noethen a Hiilff kommen. contient certaines amplifications de détail, mais le fond en est tout $\dot{i}$ fait le même.

Nons venons d'énumérer les ouvrages, contenant notre miracle, qni ont sûrement utilisé la version du Speculum historiule. Mais dans plusiem's antres cas, oì la source du miracle n'est pas expressément indiquée, il est naturellement impossible de dire si lauteur a puisé dans Tincent de Beanrais, ou bien s'il s'est servi d'une des collections de „miracles de la Vierge" d'où Vincent de Beanvais lui-même ar tiré sa version de notre miracle. Il nous fant done nous contenter d'avoir indiqué cette alternative, sans pouroir, pour chaque cas spécial, résondre la question d'origine.

Mentionnons d'abord quelques versions latines, datant encore dn XIII ${ }^{\mathrm{e}}$ siècle.

$1 \mathrm{~V}$. dans la première édition, de $1603, \mathrm{pp} .240 \mathrm{~b}-242 \mathrm{~b}$ (tom. I, dist IV, ex. III). Tans la seconde édition, de 1605 , notre miracle se lit pp. $236 \mathrm{~b}-238 \mathrm{~b}$. W'autres éditions ont été indigúes par A. de Backer, Bibliollièque des écrivains de la Compagnie de Jesus, nouv. ed. (1869-1876), t. If, p. 1012, et T. F. (rane, Proccedings of the American Philos. Sosiety, t. XXI (188t), p. 61), note †. Quand M. (rane clit (owvr. cite, p. 60, et The Fxempla - - of Jacques de Vitry [1890], pp. LXXV-LXXTT) que cet onvrage differe dn siperulum exemplorum lar l'arrangement des exemples, qui seraient placés alphabétiquement dans le Magnum speculum Exemplorum (cp. aussi Quétif-Echard, Scriptores ordinis praedicatorum recensiti — —, t. I [1719] p. 185 b), ainsi que par la manière libre dont Johannes Najor traite le contenu de loriginal, il y a évidemment erreur. Le Magnum Spcr. Exempl. (du moins dans les éditions de 1603 et de 1605) ne différe essentiellement du spec. exempl. (éd. 1481) que par l'addition de 137 nouveaux exemples par ordre alphabétique, en guise d'appendice, et dun Tractatus par le Père Johannes Diestemius.

2 V. Quetif-Echard, Seriptores ordinis praedicatorum recensiti $-\ldots-$, t. II (1721), 1. 388 a.

3 V., dans l'éd. de 1612 (Trevigi), pp. 111-8. Trois éditions de cet onvrage avaient paru it Florence avant 1594; v. Quétif-Echard, endroit cite.

+ V t. I (éd. 1687), pp. 213-23. („Der Iritte Titel. Von der Mutter Gottes. Die achto History"). Dans l'éd. de 1732 (Augsbonrg et Dillingen, in $4^{\circ}$ ), ce miracle mantue. 
Dans son ourrage intitulé Tructatus de diversis materiis predicabilibus orlinatis et distinctis in septem protes secundum septem doma Spiritus Sancti etc. (appelé brièvement: Liber de septem donis), le dominicain Étienue de Bourbon (Stephanus de Borbone; † vers 1261) ronue me version raccoureie et un peu altérée de notre miracle. On la lit daus l'ouvrage de A. Lecoy de la Marche. Anecdotes historiques, léyendes et apoloynes tirés lu recucil inédit dEtienne de Bourbon (1877)'

Épisodes: (a) l'cmpereur, qui quitte l'empire pour des affaires, laisse sa femme sous la garde de son frère; la scine de l'emprisonnement manque; l'empereur frappe sa femme des mains et des pieds; (b) lim. piratrice se defend "cum pugnis" contre le frire du seigncur; (d) l'on n'apjrend pas comment limpératrice vient de l'ile oủ elle a itci díposcic; $(f-g)$ le seignenr et son frère malade viennent chez limpératrice.

La version d'F́tienne de Bourbon a été la source de la version très abrégée du miracle qui se trouve dans l'ourrage connu sous le titre: Liber de abundantia exemplorum, attribué sans raison, dans l'unique édition (sans date, mais probablement de l'année 148(1), à "Magister Albertus magnus. Ratispa. episcopus" 2. Le véritable anteur en était sans doute Humbert de Romans ( $\dagger$ en 1277) ${ }^{3}$. Notre miracle se trouve dans la septième partie: "De timore periculorum" .

Beancoup plus près du récit de Vincent de Beauvais que la version d'Étienne de Bourbon est la version donnée par Étienne de Besançon ( $\dagger$ en 1294) dans son Alphubetum narrationum. Dans cet ouvage, qui n'a pas encore été édité, le miracle se lit sous le titre: Regina quanto dignior, lanto magis castitatem suam servare debet ${ }^{5}$. Les différences avee le miracle latin sont tout ¿̀ fait insignifiantes, mais le récit est très condensé ${ }^{6}$. LiAlplabelum narratiomum a été tradnit en anglais et en catalan. La traduction anglaise de notre miracle nous est comnue par un ms. du $\mathrm{XV}^{\mathrm{e}}$ siècle,

1 Pp. 115-7 (no. 136). Dans louvrage d'Étienne de Bourbon le miracle est rangé dans la seconde division: ${ }_{n}$ Le eis que pertinent al donum pietatis* (Tit. VI: No beata Maria: Quod servientes sibi a tribulacione liberat)

2 ("p. L. Hain, Repert, bibl., t. I, partie I (1826), p. 53 b. 484.

3 V. (quitif-Echard, Seript. Ord Pravl. (1719-1721), t. I, pp. $147 \mathrm{~b}-148 \mathrm{a}, 186 \mathrm{a}, 430 \mathrm{~b}$; T. F. Crane, dans The Irademy, 18s6, 20 févr. (no. 720), p. 133. (1). cependant Edw. Schröder dans Zeitschr. f. dentsches Altertum, XLIT (1000), 425. - Il y a, dans l'Histoire littéraire de la France, XXIX (1885), 546--51, un article de B. Hauriau sur lourrage en question.

4I n'y a pas de pagination, mais c'est fol. $59 \mathrm{r}^{0}-59 \mathrm{r}^{\circ}$ - Ce qui prouve directement que la version du Liber de abudantia excmplorum est basée sur celle d'Étienne de Bourbon, c'est gune dans les leux l'empercur frapue sa femme „des mains et des pieds" et que l'impératrice se défend anssi "avec les poings“ contre le frire de son sauveur.

5 Y. les mss. Paris, Pibl. nat., f. lat. 15!913, fol. $74 \mathrm{r}^{\circ} \mathrm{a}-74 \mathrm{r}^{0} \mathrm{a}$; Paris, S:te Gen. 546 (année 1328), fol. $169 \mathrm{r}^{\circ} \mathrm{b}-170 \mathrm{r}^{\circ} \mathrm{a}$; Londres, Hrit. Nus. Harl. 268 (XV:c s.), fol. $178 \mathrm{v}^{0}-179 \mathrm{r}^{\circ}$. Pour dautres mss. contenant l'Alphabetum narrationum d'Etienne de Resancon, v. T. F. C'rane. The Exempla - - of Jacques de Vitry (15!0), 1. LXXI, note*. - Nous publions la version un ms. de la Kibl. nat, f. lat. 15913, dans notre Appendice (J).

- comme on lit dans les mss., apres le titre du miracle: „Ex Miraculis Beate Virginis", il est fort probable que l'autour a puisé dirertement dans une collection de miracles de la Vierge, et non dans Vincent de Beaurais. 
on le titre latin est encore conservé ${ }^{1}$. La traduction catalane, qui date de la fin dn $\mathrm{XlV}^{*}$ olt dn commencement dn $\mathrm{XV}^{\mathrm{c}}$ siècle ${ }^{2}$. a été imprimée. sinns date ni lien ${ }^{3}$. sons le titre: Recull de eximplis e miracles, gestes e faules e altres ligendes ordenates per A-B-C, etc. Totre miracle $y$ est annoncé de lia manièse snivante: Mirucle que la ierge Maria fou a Lemperadrin muller del Emperador de Roma, sigons ques recompte en los miracles de la verye Maria mure de Jhesuchist, après quoi snit la rubrique latine: Regina quanto dinior. [dinior?], tanto magis castitatem suam debrt obseruaret.

1 a dernière version latine dn miracle que nous ayous à mentionner est un comt r'écit en vers rythmiqnes de Jean de Garlande (Johamnes de Garlandia) faisant partie de son poène non édité Stellu maris de Mriraculis Beale Marie Tirginis", composé vers $1245^{\circ}$. L'exposé de Johannes de Giulanilia semble bien basé sm le miracle latin. mais ne raconte que très sommairenent notre légende ${ }^{7}$.

Passons maintenant anx versions en langues modernes.

En framgais, nous avons dabond à mentionner la version que donne Jean Miélot (milien dn $\mathrm{XY}^{\mathrm{e}}$ siècle) dans ses Fivaeles de Nostre Dame. Notre miracle (no. XXIX) y apparât sous le titre: Tune grant dame de Romme qui fut par deux foiz faulsement acusee de adultere et de omicide, la quelle par la grace de la vierge Marie, a qui elle recourroit, fut deliuree et meseruers. Cette rersion ne diffère guère dn miracle latin:

Persominges: (1) un grand seignenr de Rome; (t) Heros.

1 Ms. Brit. Mus., Add 25719 , fol. $157 \mathrm{r}^{0}-\mathrm{r}^{0}$. Le miracle commence ainsi: "We rede in pe milacles of our ladie how somtyme in Rome per was ane Emperour pat harl a fayr wyfe and a chaste".

2 V. T. F. Crane dans les Procedings of the Amorican Philos. somely. XXI (15st), fi2.

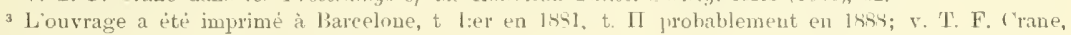
The Exempla - - - of Jacques de Titry. p. "T, note*.

+ T. II, I]). $20 \mathrm{I}-6$ (no. DX(')

s Les deux premières strophes du poime, ainsi yue la dernière, ont iti publites par A. Scheler,

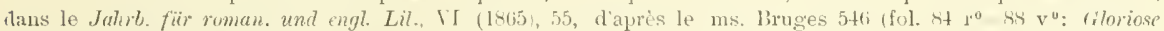
virginis mirucula). M. Hauréau a encore communirui la premiére strophe, d'ajrís le mêmo ms., dans les

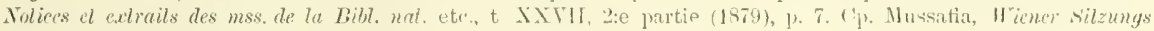
ber., philos.-hisl. Cl., t. ('XIX (1589), mém. IX, P1. 6-7.

- $Y$., pour cette date, Ward, Catal. of romanres, II (1593), 699.

T $V$. le ms. Londres, Brit. Mus. Roy \&. ('. IV (XIT:e siecle), fol. $18 \mathrm{r}^{\circ} \mathrm{b}-\mathrm{v}^{\prime \prime} \mathrm{l}$ (le texte est accom jagné de gloses latines diune main postritienre). Le miracle est contenu en onze strophes et demie, rom

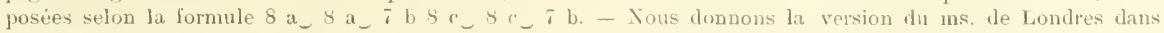
notre Appendire $(\mathrm{K})$.

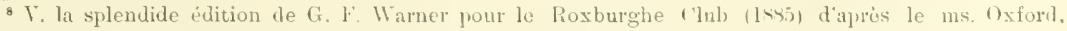
Douce 37t, 19. 23-4 (texte imprime) et fol. 31 a - 37 a (reproduction aututypinge). Fol. 30 b if $y$ a une magnifipue peinture en grisaille représentant lemprisounement dans la tour eu trois scénes conscicutives, figurant sur le même plan. - Pour lidition du lioxburghe ('Jub et les mss. contenant lienvre de Michot.

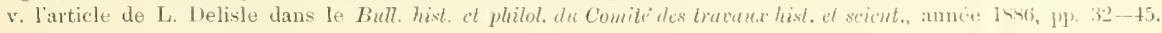


Buaromp plus bloigné du miracle Jatiu est lal version fortement abrégée de la

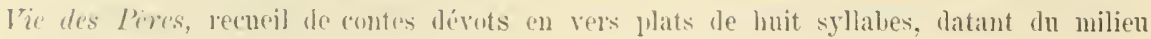

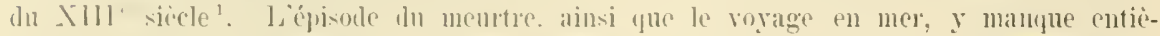

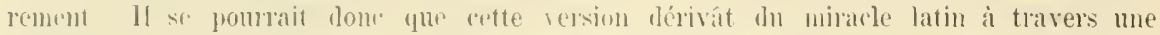

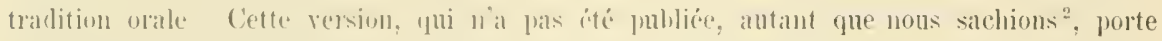
dams les manuscrits diffépentes rubriques, rapjelant jhus ou moins celles du miracle de

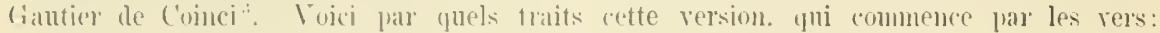
"Fouk est qui acroit sul ses piaus.

'Pandis comme li jeus est bians" - - -

1. pour rette date, E. Sillwan dans liomanin, XIII (1454), 35\%.

${ }^{2}$ Elle nest pas dins les ancienne's iditions de la lö des lirres. Nous arons consulté celle de

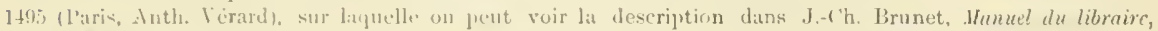
t. 111 (14(i2), rol. 163.

2 Nous publions cette rersion dans notu Appentie (1.) d'ajues le ms Paris, Bibl, nat., f. fr. 1546

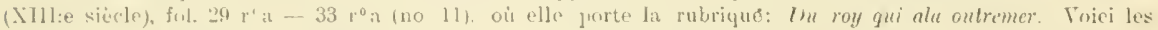
autres rubriques iue nous connajisons:

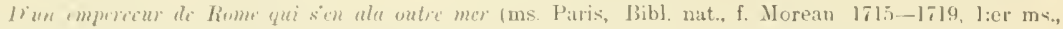

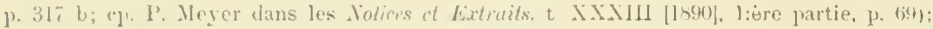

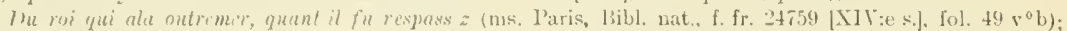
11. lemperenr qui ale outir mer it lessa sa fimme in la garde son frere (ms, Paris, laibl nat., f. fr.

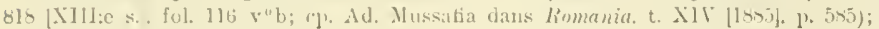

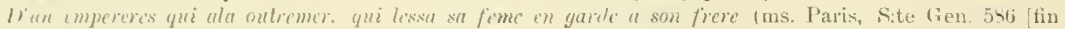
du XIll:e s.]. fol. 1:2 $\left.\mathrm{r}^{0} \mathrm{a}\right)$;

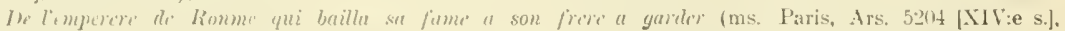
fol. $136 \mathrm{v}^{\text {"b) }}$ :

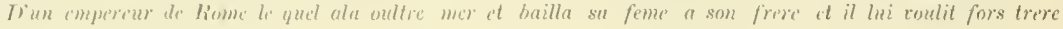
(ms. L'aris, Ars 5-3lt [XV:e s.., fol. $131 \mathrm{r}^{0} \mathrm{a}$ );

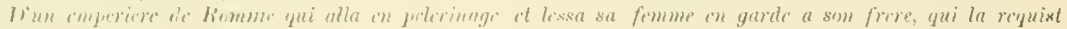

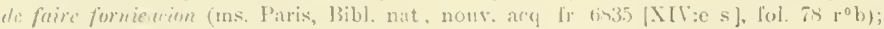

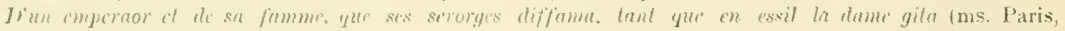
13ihl. nat., f: fr. 2.ivt3i [XIJI:e s.]. fol. 4] volu):

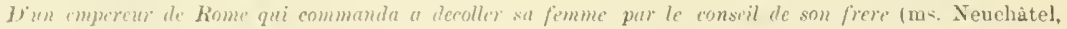

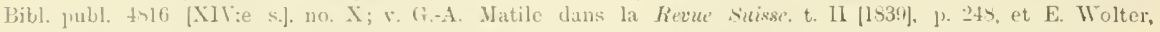

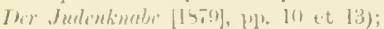

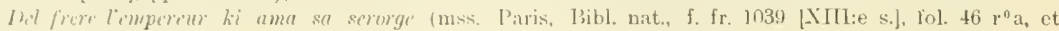

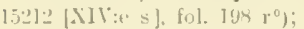

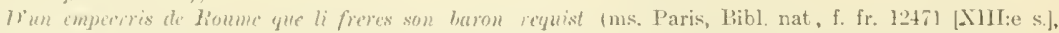
fol. $\left.2(1)+\mathrm{r}^{\circ} \mathrm{b}\right)$;

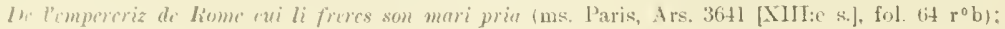

Ih. l'mperix de liome qui fut mal mise par le lrayson de son serorge (ms. Paris, 13ibl. nat., f. fr. 1545

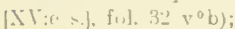

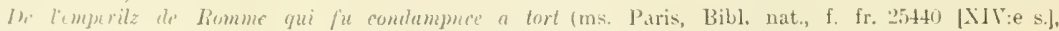
fol. $1\left(\mathrm{ii}, \mathrm{w}^{0}\right) \mathrm{i}$

Ih. l'empreälz de lomme qui fusl a moult grant tort comelempues (ms. Paris, libbl. nat., f. fr. 154t (XIT:e s.J, fil. $57 \mathrm{r}^{\mathrm{n} h}$ ):

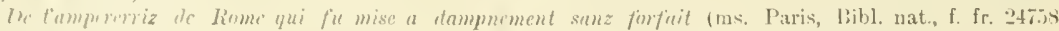

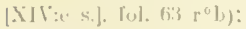

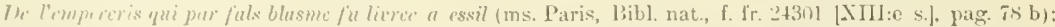

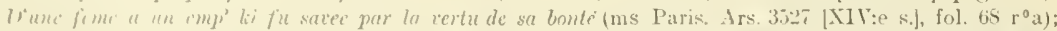

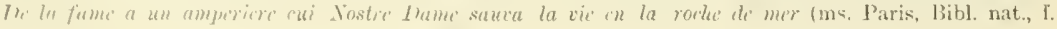

fr. $1 \times 17$ [X]: : s.]. fol. 7 ti $\mathrm{r}^{0} \mathrm{a}$ ).

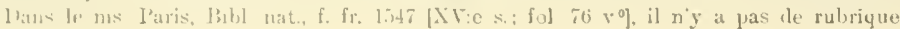


differe dı miracle latin:

Personnages: (4), (5) et (i) manquent. Lisodes: (a) e'est à cause d'un vou, ficit pendant ume mala die, que l'empereur va visiter les lienx saints; il confie le gouvernement de l'empire i son frère, sous la surintendance de sa femme; il nest pas dit que ce soit par ruse que limpérntrice enferme le beall-frère; celui-ei dit que cest l'imperatrice qui l'a enfermé; c'est à trois ehevaliers que l'empereur donne l'ordre de tuer limpriatrico; les ehevaliers se laissent attendrir et déposent l'impératrice directement sur le rocher au milieu de la mer; lépisode (b) manque; (e) le premier que l'impératrice guérit, artivè à la terre ferme. est le seigneur du pays: ( $f-g$ ) les trois ehevaliers qui araient épargné la vie de lia souveraine sont réeompensés; l'imperatrice ne se fait pas nonne.

De cette version T'.-e.-B. Le Grand (Legrand d'Aussy) a donne une analyse.

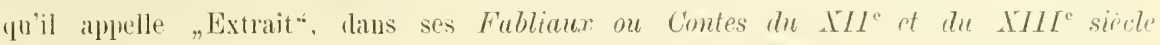
sons le titre: De la bome Imperatrice qui gavda loyalement lu foi du mariuge, on de l'Empreur de home qui fit le coyage d'outromer. Cette analyse reproduit firlelement la version de la Tie des Pères.

Dans me note ajoutée à la fin de som analyse. Legrand d'Aussy lait saroil que "M:lle de la Rochegnilem ${ }^{2}$ a fait de ce Conte, sons le nom d'Adéluädr de Hongrie, 11n Roman". En effet, e'est bien smr le „eonte dérot" "gu'a analysé Legrand "l’Ansy que parait être basé le roman insipide de M:lle Laroche Gruilhen († m 1710)". Voici les mincipanx traits par lesquels il diffère de la version de la lie des Ières:

Prrommages: (1) Ladislas, roi de Hongrie; (2) Arleleide, fille du roi do Bohême; (3) Venceslas. Episodes: (it) le roi litit une expélition guerrière contre le Mahométan Imurath; Veneeslas a été auparavant amoureux d'Hildegrade, cousine d'Adeleide: Veneeslas déclare son amour, non seulement de vive roix, mais aussi par une lettre; l'emprisonnement dans la tom manjue; sans aroir revu sa femme, Ladislas donne à un chevalier, Edmond, l'ordre de la tuer; Adeleide se rend avec Eilmond et Hildegrade it un châtean solitaire oủ elle croit reneontrer son mari; fuand Edmond vent accomplir sa mission. Adeleide montre la lettre compromettante de Vencesłas; Elmond épargne la reine, mais annonce i Ladislas sa mort, lui montrant en nême temps la lettre de Veneeslas; le roi, désolé de sa eréculité, chisse son frére: (f - g) Hildegrade fait savoir au roi gu'Adeleide vit; le roi accourt an ehateau; Venceslas reçoit son pardon à condition l'épouser Hildegrade+.

1 V. l'èl. de 1781 (in-120), t. V, pp. 16t-9); 3:e ed. (1429, Fablinux ou mules, finbles el romans thu XII:e it du XIII: sierle), t. V, pp. 125-9. Dans le volume anonyme de 1781 (in-8\%), (1ui porte le titre: Conles dévols. Fables al Romans aneiens: pour scrvir de suite aux Fabliaux, notre conte est donne pp. 115-9 (ce volume forme le tome IV de l'édition de 1779, parue en trois volumes). - Une traduction anglaise de cet "extrait" a èté donnée par M. W. A. Clouston dans les iriginals and Analogucs of some of Clanecr's Canterbury Tales (1888), pp. 397-9.

2 Lans l'éd. de 18:9: „Madame de La Rocheguilhen".

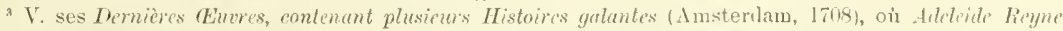
de Hongrie ocenpe pp. 66-102. D)ans une edition de lannée suivante (Amsterdam 1709, in-12), qui porte le titre: Histoires curieuse's et galantes ou Dimières curres de Hademoisclle Lu Roche-Guilhen, notre histoire se lit t. II, pp. 1-50 ( Adeleide. Reine d'Hongrie. Nonvelle Historique"). Il y a encore une troisième édition de ce livre, de 1711 (Amsterdam, in-12), portant le titre: Eiweres diverses, contenunt quelques histoires galun-

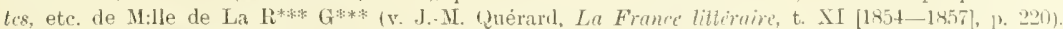

+ Un résumé de ce roman a été donné par l'Abbé de La Porte dans l'Histoire littêraire dis fr'mmes Frangises ou Letres Historiques et Critiques - - - par me Soriéte de liens de Lettres, t. III, Paris 1769, pp. 91-2 (Lettre VII). 


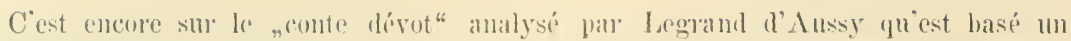

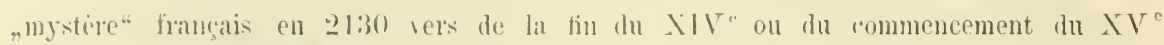

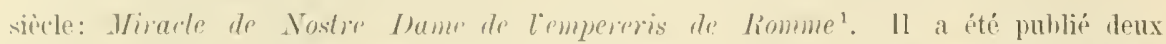

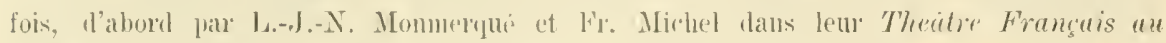

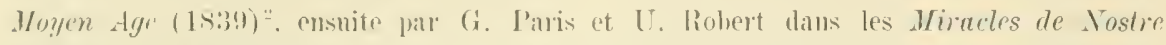

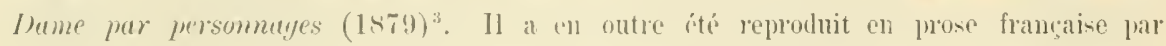

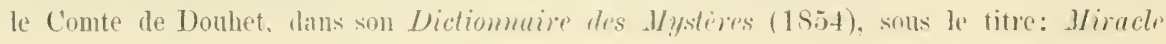
de l'Imprivatrice romaine!. Enfin, l'etit de Julleville l'a analysé longmement daus son Histoire du Theatre en France $(1890)^{5}$. Cette version diffère. pour le fond du récit, très peu de la version de la lie des Peres ${ }^{6}$ :

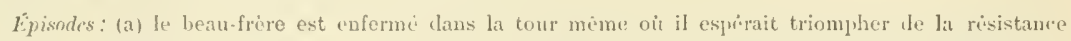
de l'impératrice ; (d) Dien lui-mime emoie lit sainte Viergo an seconrs de l'impreratrice; c'est à Naples jue

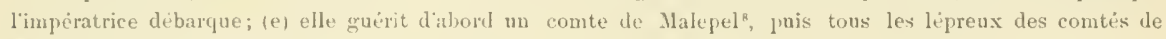
Célanne, de Malepel et de Fondi.

En Italie, uotre miracle a eu un déreloppement encore plus remarquable yu'en France.

Mentionums "labord un long poème ell "ottava rima", L'Uramia overo La. Costante Domu, par Don Felice Passero, "Abbate della Congregation C'assine se" (imprimé en 1616 à Naples „appresso (rio. Domenico Ronugliolo", in-8 ${ }^{0}$ ). qui a pris pour source le miracle latin, mais y a introrluit nombre d'amplitications de détail et

I Un passige du mystire vi il est question de Rohert d'Anjou, roi de Naples, montre ipe le mystére est postérieur a lavinenunt de ce rui (1309); cp. Petit de Julleville, Lex Mysteres. II (1890), $295-6$.

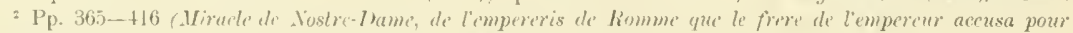
In fire destruire, pour ce quidle viewoit rolu faire se voulent; it depuis devint mesel, de la dame le garit quant il of righy son meffail). If y a une traduction en prosu francaise en regard du texte original. - ("est naturellement par erreur que les auteurs disent que ce mystère a tite emprunti an conte de fiautier de loinci

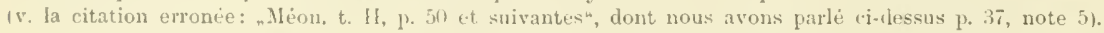

3 T. IV, Pp. $237-313$ (no. XXVH: Miracle de IEmpereris de liomme).

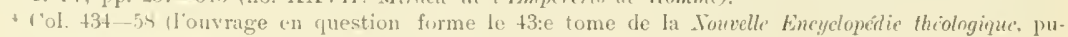
blice prar liblé Mligne). Douluct a divisc son texte en cinquanle-scpt nscènesn.

s Prem. partie: Les Mystèr's, II, 294-6.

- M. L. Voigt Ifie Miraliel der Pariser Hs. S19, welche cpische Stoffe behandeln, auf ihre Quellen untersucht [15r3]. P]' 6$]$ क) a analysé en ditail notre mystive, en le comparant à la version de fiautier de ('oinci, "qu'il regarde a tort comme la source ilu mystire (p. 61).

" ('e rapprochement entre notre mystere et la source latine lainsi 'pue la rersion de Gautier de

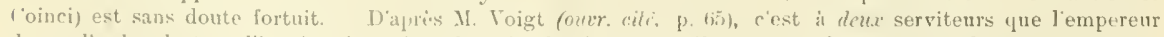
donne l'ordr de tuer l'imperatrice: dans le miracle diamatisé il y a rependant, comme dans la rersion de la Vie des l'òres, trois chevalier's pui sont charges de cette mission.

B ("est sans doute un nom tiré de la maladie du comte; cp. Mirurles de Tostre llome par personnages. publiés par G. Paris et [. Robert, t. VIII (1\$, 33, p. 302, s. v. Malepel. 
a, en ontre. moditié complitement la scène dn meurtre?. Toici les principanx traits par lesquels cette version diffère du miracle latin:

Persomnages: (2) Crania; (3) Manlio; (4) Eliodèmo; (5) Ennanno. Épisnlex: (a) l'empereur met sa femme sous la protection de Maulio; Manlio raconte à son frère revenu que cest l'imperatrice qui la tenu enfermé dans la tour; c'est a toute une troupe de valets fue l'empereur livre sa femme; (b) Ermanno reut prentre Trania de force et tue fe petit garçon (Alessio), yuand il se nut à crier, aprés quoi il s'enfuit, laissant le couteau auprès d'Urania: (1) les marins, effrayés par une violente tempéte, déposent Urania sur un ilot, oi elle passe ensuite dix années, protégée par la sainte Fierge et ses anges; c'est un bateau conduit par une main invisible qui porte Lrania á la terre ferme.

Lal rersion italienne qui, aree le poème de Passero, se mapluoche le plus du miracle latin est aussi un poème en „ottava rima“. La historia ti Flavia Imperatrice, dû à la plume de Gioranni Briceio (1581-1646). Ce poème a parm nombre de fois sous forme de feuille volante ${ }^{2}$. Dans une des éditions que nons connaissons il est dit que le sujet dı poème a été tiré des Miracoli della Madomm. "Libro secondo, Miracolo primo" ${ }^{3}$, done probablement de la version de silvano Razzi (v. ci-dessus p. 41). Dans une antre édition ${ }^{+}$, la source indiquée est ,Miracoli della Madoma, Lib. ‥

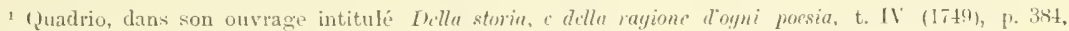
signalait déjà le poéme de Passero. In exemplaire le lédition trés rare de 1616 se tronve à la Biblioteca Nazionale de Florence. N:Ile Aline Pipping, de Helsingfors, a bien voulu, pendant un séjour à Florence, nous faire un résumé détaillé de louvage en question. Le poème se compose de yuinze "ranti et rontient plus de neuf cents .stances". Au commencement il y a un "Argomento dell'Opera ", à la fin duquel lanteur renvoie aux versions de Vincent de Beauvais, du "Wiscipulus" et du "Giardino dessempi”, ouvage yue nous ne saurions identitier.

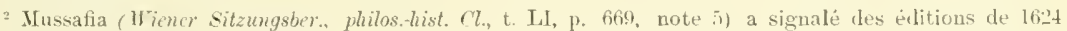
(Viterbo), de 15122 (Bologna) et de 1562 (Prato), ainsi gu'une édition sans date (Treviso). Nous sommes a méme llajouter les éditions suivantes dont nus avons pris connaissance: 1) Istoria di Flaciu Imperatrice, La quale fu liberata dalla Gloriosa Torgine Harie da molte tribulazioni, e strani aecidenti, e ritatto wel sue primiero stato; s. I. ni d. (1700?), in - t $^{\circ}$ comme autenr est nommé (iouambatista Pittore liomano), S1 strophes, $8 \mathrm{pp}$. (Il $\mathrm{y}$ a une gravure représentant un navire qui salproche du rivage; sur le rivage denx caraliers couronnés avee leur suite: plus loin ume tour; 2) Lstoria di Flavia Impratricr, La quali fu laberatu slullu

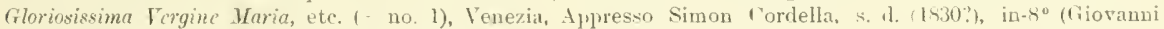
Briccio, Pittore Romano, est nommé comme auteur), 81 strophes, 23 l'l). (p. 24 il y a un sonnet en l'lonne'ur de la Vierge ; 3) Istoria di Flavia Imperatrice La quale fin liberata dalla Gloriose I: Maria cla molte tribulazioni, e strani accidenti, con farla ritornare nel suo primiero stato; Nella stamperia di Gaetano Eboli (Naples $1800 \%$, in-120, Si strophes, 24 pp. (Plusieurs gravues sur bois, dont celle du cotnmencement représente un roi et une reine sur le tróne, avec une personne de chaque cóté); tl Istoria etc. (a peu prés = no. 3), Napoli, s. d. (1820?), in-120.81 strophes, 24 pp. (liravures comme dans no. 3); 5) L'Isteria di Flavia Imperatrire La quale fu liberata dalla Giloriosa Vergine Maria ia tante tribolazioni. carata dal Libro de Miracoli della Molonn: Lucca, s. d. (182(1), in-12\%. 81 strophes, 24 p). (Gravure sur bois représentant une femme tenant par la main un petit enfant, un roi yui met sa main sur la tête de l'enfant, une reine et encore une cin-

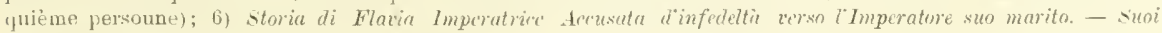
patimnti, e come fosse rironosciuta da tutti la sua imocenza: Firenze, 1579, in-120, 65 strophes, - -4 pp. (Gravure sur bois reprékentant un roi et une reine sur le trone, avee trois hommes derrière une table): 7$)$ storia, ete. $(=$ no. (6), Firenze. 1599 , in-12, 65 strophes, 24 pp. (Gravure $=$ no. 6); cest le no. $x$ dune Collezione $\pi i$ $30 n$ libretti "ai Storie. di Canzonette. di Giunchi a da videre a 5 centesimi luno a seelta.

3 C"est le no. 2) de la note précidente. Dans cette édition, il y a également un renvoi is Vincent de Beauvais et an "Discipulus".

( ("est le no. 5) de la note avant-dernière. 
Mir. s", recheil de miracles que nous n'avons pu retrouver. Les dirergences entre la rersion de Briceio et lo miracle latin sont de pen d'importance:

Personnorges: (3) l'lavia; (3) Gillirano. Eipisolts: (a) lo bean-frère est enfermé dans la tour haute du ('apitole; il raconte a lempereur fue cist limpreratrice qui lia temu enfermi; (d) Flavia guérit deja un lipreux sur le navire yui l'rmporte de la petite ile; $(g)$ l'lavia ne se fait pas nonne.

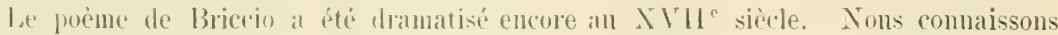
une . Rinplutesentazione per Mnsirä en trois actes de F'rancesco Beverini, intitulée La Flacia Imperatrice (Lalermo lifig, .pes Bua e Camagna", in-120), laquelle ne diffère pas essenticllenent de sia source. si ce n’est par l'omission de la scène oì l'ikstasin est gueri'. Mentionnons senlement les divergences snirantes:

Persmmagis: 1) ('esare; (t) 1'lodio; (i) Agriplu; (7) un marin. fipisodes: (a) c'est un bouffon, ('rullo,

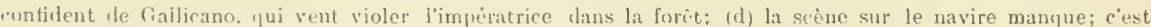
sur l'ile déserte que le marin veut faire violence a l'impératrite; il est mis en fuite par des auimaux saurages yui prolegent Flavia; if $g$ ) il n'est pas yuestion de la guérison d'Agrippa.

Pent-rotre une antre Rappressutazions sucra, intiulée Santa Flaria (Venezia

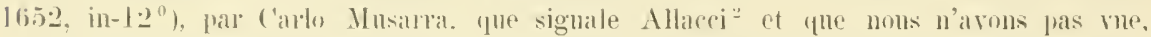
est-elle également hascre smo le poème de Briceio. Mentionmons entin que G. 'Tigri. dans ses canti popolori toseuni (1sjti), aftirme que, le son temps, on jonait encore dans la province le drame de Flavia imperatrice?

Si les versions de l'assern et de Briccio ont encore rouservé mósque intact le sujet du miracle latin, nue troisiène version. I'Istovia di c'untu Gruglielmu, tirée plus on moins directement du miracle latin', a supprimé lemprisonnement dans la tour et lus tentatives anourenses des marins, mais a, lantre part. ajonti une introduction étrangère an thème primitif du milacle, ainsi que différents détails nonveanx. La légende de sainte fruglielma est conserve en lenx rédactions principales en prose, pareilles pour le contenu, mais lifférant par la forme. L'mne de ces rédactions. la plus anciemne les denx (elle existe dans des mss. du XIV" sjècle), est divisée en douze chapitres. portant rubrique. Elle a été imprimée diverses fois, leruièrement, en 187s. par les

M:lle Aline Pipping, de flelsingfors, a en l'ubligtance de nous faire un résumé détaillé de la mapprantazione de lieverini d'apres l'exemplaire fui se trauve daus la biblioteca Nazionale de Florence - llans l'édition de 175.5 de la Thammaturgia d-Alincei (col. 361, no. i), lieure de Beverini se trouve signalé sous le titre de Flavia Imperarlice. Peut-itre que ciest In mime nuvrage que Mandosio, dans sa

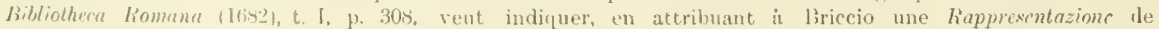
Flavia Imperatrice.

2 Irammaturgin (él). 1755), rol. $361|+|$.

${ }^{3} \mathrm{~V}$. Tigri, ouer, riti. 1. XXXl1.

" Liun des mss. qui eoutiennent la légende en question (ms. Lonulres, Brit. Mus., Add. 1005l) commenec ainsi: "In questo libro e descripta la compassionenele hystoria de la beata Guiclma Regina de Ongaria layuale se recita in $L i$ miraroli de la uergene Maria." 
soins de M. G. Ferraro ${ }^{1}$. Linteur de cette rédaction, qui est en dialecte toscan, itait

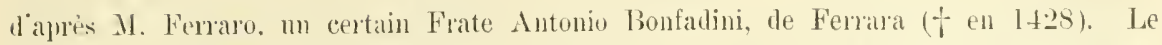
titre de la rédaction de Bonfadini est: Istoria de Sunta Gralielma fiola dro Re dungalteria. et moylie delo hi dunguria”. Lautre rédaction (en dialecte vénitien) est divisée en trente chapities et a jour anteur l'abbé Andrea Bono ( $千$ arant 1466$)$. Hille a été reprodnite plusiems fois. Nons avons pris connaissance de cette rédaction dans le ms. Londres, Brit. Mus., Ade. 10051 ${ }^{3}$ ( $\mathrm{XV}^{\mathrm{c}}$ siècle), ò la légende commence ainsi: In questo libro e descriptu la compassionenele hystoria de la beala Guielma Regina de Ongaria laquale se recitu in Li miracoli de la uergene Maria. - L'Istoria di Santa Guglielmu diffère par les traits suivants du minacle latin:

Vite di s. Guglielma Regina IThgheria e di S. Eufvasia vergine Romma, seritte da Frate Antonio Bonfadini (= no. 159 de la collection seclla di curiositic letterarie incdite o rure dal serolo X7II al XIII in Appendice alla Collezione di Opere inedite o rare), Ip. 1-67. Jans cette édition il ny a pas de division en chapitres

* Tous navons pas vu diautres éditions de la rédaction attribuée à Bonfadini. Les mss. que nous connaissons donnent les rubriques suivantes:

Leggenda di santa Gughelma figluolu del re dinghilterra e moglic del re di l'ngheria (v. A. Mortara, Catalogo dei manoserilli italiuni che sotto ta denominazione di Codici Camoniciani llalivi si consereno nella Biblioteca Bodlietna a Oxford [186t], p. 21+b [ms. 215, no. 23]);

Legenda de sancta Guilgelma figliola dello te de Enghelterra et doma dello re de I'ngaria (ms. Paris, Bibl. nat., f. ital 665, fol. 10 [Xll:e s.]; ep. G. Mazzatinti, Inventario dei manoserilti italiani delle biblioteche di Francill, t. I [1856], p. 127);

Leygenda di santa Guglielma fighola del Re dinghilterra. la quale fu maritata al Re d'Thingeria (v. J. Morelli, I Codici Manoseritti volgari della librerin Naniane [1766], p. 69 [eod. LXTII, no. 6]: Fr. Palermo, 1 Manoseritti P'alatini di Firenze, t. 1 [1853]. p1. 259 [cod. (XXXI (XIF:e s), no 1] et 26t [eod. CXXXII (XT:e s.) no. 4]);

Legienda di Santa Guglithnn, figluola del re allighilterra (v. A Wesselotiky dans la Rivista bolognese di srienze, lettcr, arti e scuole, t. I [1567]. p. 423 [eorl. magliabech., cl. VII, 11s\$])

Les rubrịues des chapitres de ce dernier ms. out eté données, avec une analyse sommaire de la légende, par Wesselofsky dans l'art. cité. 11. 433 6 Nous donnons ici les rubriques des chajitres du ma. I'aris, J3ibl. nat, f. ital. 665 , lesquelles diffèrent complètement de celles données par Wesselofsky: I. Incommena (sic) la legenda de saneta Guilgclma figliola dello lie de cnyholterra. i donna dello he de enyaria: II. Cömo seä Guilgelma pidieaua al suo marito la passione de xpō. F iuitaua lo dhe uisitasis laterra saneta: HI. Como

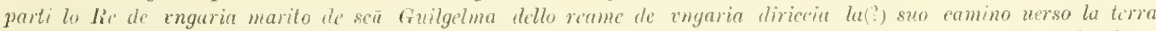
sancha; IV. Cömo lo Re de engaria approxima languria retornando da ienasalem; V. Como cl fratello dello Re a

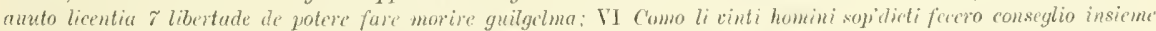

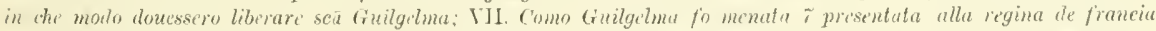

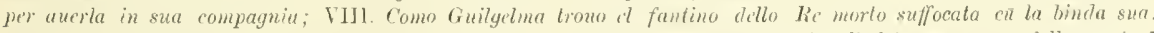

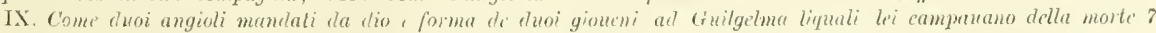
monaron lu ara caminando cel lei lie giomate finc alla masina; X. Como Guilgroma farta la sana oratione adio

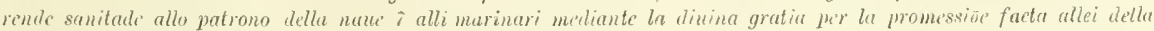

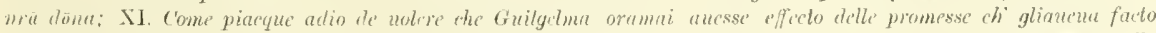

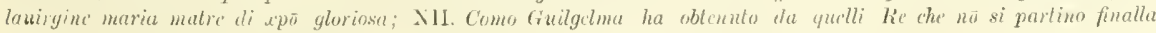

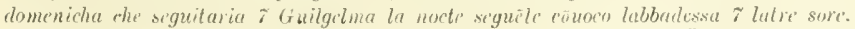

${ }^{3}$ Il y a, par erreur, dans le mémoire de Ad. Mussafia, L̈ber éine italienische motrisshe Iherstellung der

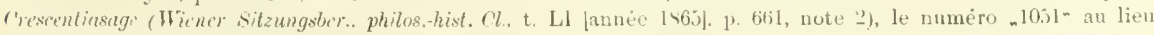

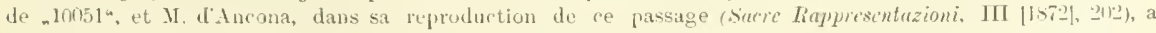
répèté cette erreur.

+ Tleux autres mss. de cette rédaction (l'un à Vérone, liautre ì Vénise) ont été signalés par Gr. Maznchelli, Gli scrittori dTtalin, t. II, part. 111 (176-), p. 167\%; cp. aussi E. A. ('igogna, Thlle luserizioni

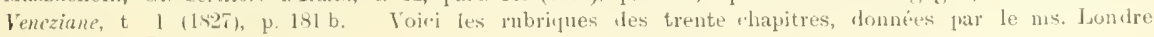


Pirsonnages: (1) le roi de Hongrie; (2) Ginglielma, tille du roi dingleterre; (4) le roi de France; (5) un sincichal du roi. Eivisorls: (a) longue introduetion, dans. laupelle il est raconté comment le mariage

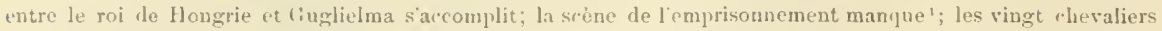
yni ont recu lordre de brûler la reine sont touchés de compassion et la laissent en vie; (b) le petit garçon est étranglé avec une nbinla" appartenant à Guglielma: "elle-ci est condamnée à être brilée; jendant la nuit, la sainte Vierge apparit et donne it Guglielma le powroir de guërir, par ses prières, tous les malades qui confesseront leurs fautes; le bucher dija dressi, les bourreaux sendorment, et deux anges, sous les traits de denx jeunes hommes, la condnisent a la mer, où un navire la recueille; (d) sur le navire Guglielma, visitée de nouveau par la sainte Viergu, guérit tous les marins, tombés subitement malades, et leur capitaine la conduit directement i la terre; (f) Jes deux criminels, devenus lépreux. viennent, acconpagnés des deux rois, che\% Guglielma; (g) Guglielmiz ne se fait pas nonme.

\section{C'est sans doute de l'une de ces deux réilations de l'Istorin di Santa Guglielma} que s'est servi le r'uré de Brunate (Côme), Andrea Ferrari, en érrivant sà l'ita di

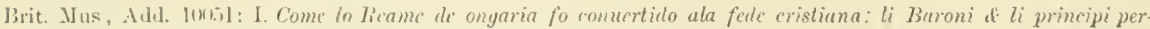
suadeua amisser lo lic del dousss tuor per sposa una domu nobilissima et bene urostumata: II. Come li ambasiatori gionti in Anglia furono apartamento cuä lo he et eñ le regina de duglia per hauer sua per sposa delo Re de

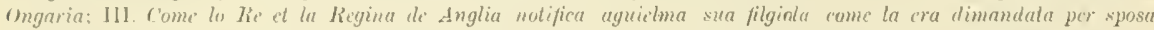

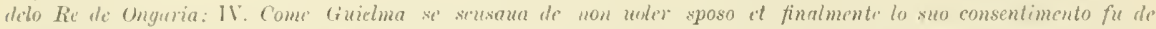

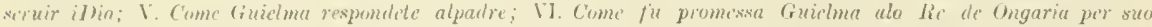
padre; VII. Come fu mandado it frudelo det lie de Onguria in anglin rum molti Baroni per contur lasposa ama.

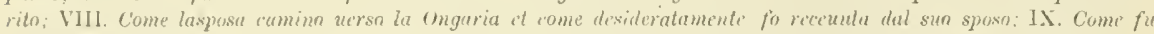

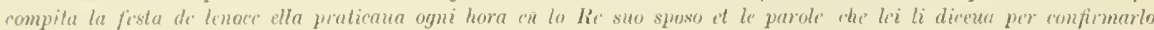

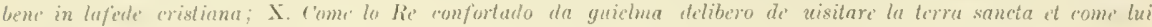

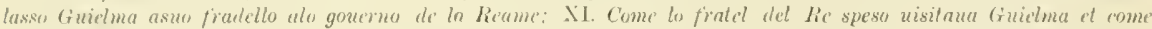

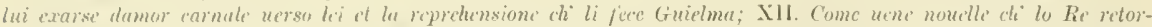

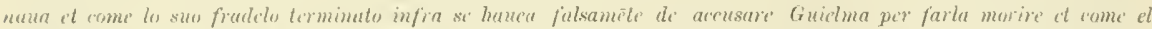
he nen pote credere tul cosa di sun dilieta spesa: XIII. Como lo Re Contaminato dal suo fratello consenti finalmente di ciuielma fusse punita; XIV. Come el fraldlo del Re rimase contente ot molto aliegro quando lui abtene

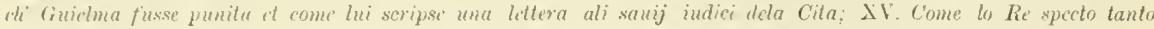

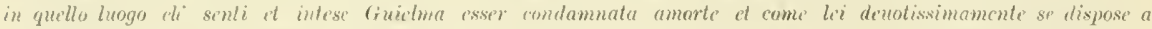

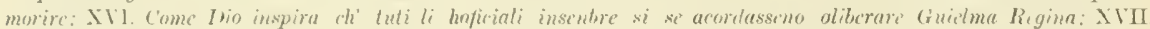

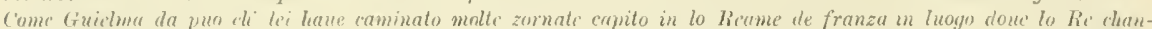

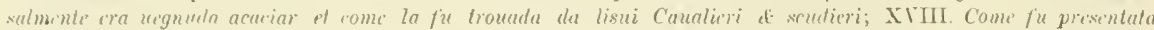

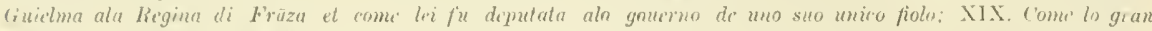

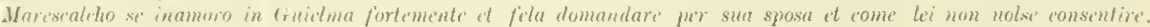

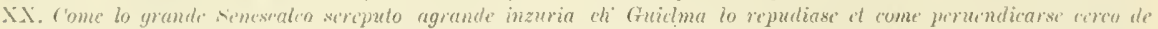

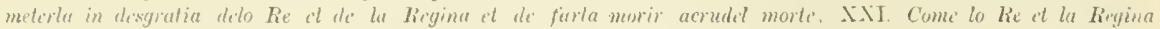

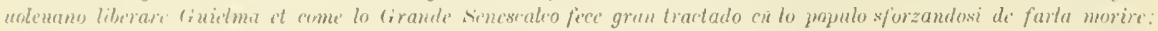

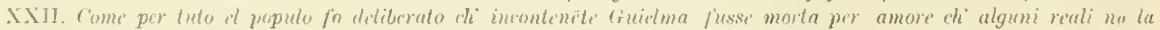

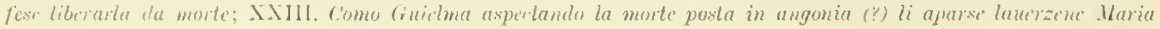

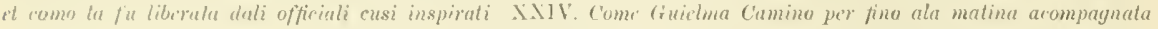

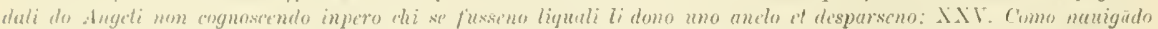

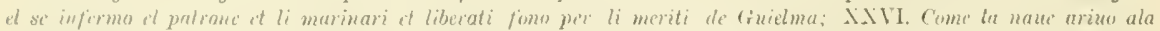

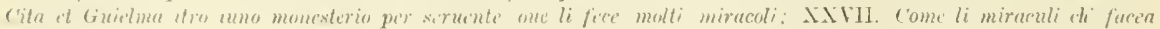

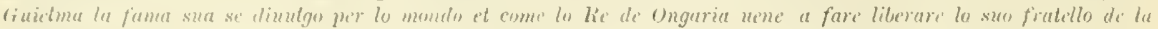

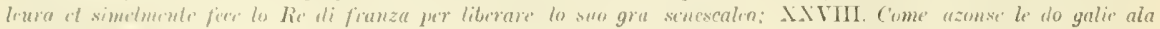

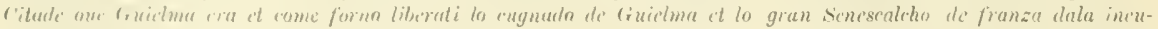

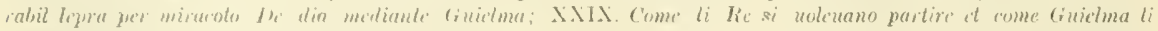

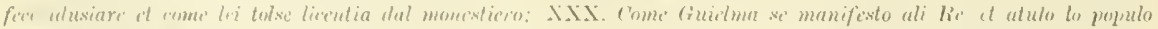

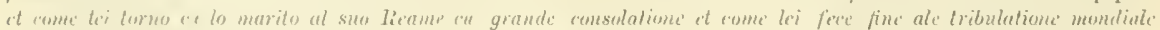

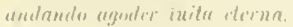

1 (1). La version d'bitienne de liourbon (1. 42) 
Santa craglielma, dont nne élition a paru a Cône en $16+2^{1}$. Nons ne comnaissons pas la version de Ferrari elle-même, mais, d'antès me analyse donuée. en 1s42, par Michele Caffi dans son ouvage Dell'Abbaziu di Chiuruvalle in Lombartiu ${ }^{2}$, elle diffère des versions de Bonfadini et de Bono an moins par les traits suvants:

Personnages: (1) Teodo. Épisodes: (a) le mariage a lieu en 795 ; (b) la sainte Vierge ne so montre pas.

Bartolommeo Gramba, dans son onvrage Delle novelle ituliume in prosid Bibliografia ${ }^{3}$, signale encore une rédaction de la légende de Santa Cinglielnua. imprimée au XVI" siècle (sans date). Cette édition, que nons n avons pas vne, porte ce titre: La Mistoria della Serenissima Regina di Polonia, laqunle due volte iniquamente fu munduta melle silve ad necidere, etc. (Qu'jl s'agisse bien de Santa Guglielma, c'est ce qui ressort de la description que donne Gambal de la gravme initiale: "Nita nel frontispizio un intaglio in legno in eni la regina Guglielma. vestita in abito di frate, accoglie i graudi del regno ed il re stesso genutlessi a' suoi piedi“.

LiIstoria di Santa Guglielmu a été dramatisée en "ottara rima” ver's la fin du XV" siècle par Antonia Pulci, femme de Bernarlo Pulci, et cette , sacra raphresentazione" paraît avoir joni donne grande renommée, timoin les nonbreuses éditions qui sn existent ${ }^{*}$. Son titre varje: La Festa di Sunta Guglielma, Fita e Mrraeuli di ranta Gugliehna, happresentuzione della Tita e Miracoli di Santa Guglirha, etc. En dernier lien, en 1872, elle a eté publiée par M. Al. "Ancona lans ses Sacre hupresenlazioni sons le titre: Kuppresentazione di Sunta Guglielma ${ }^{5}$. Ce drame sacré a de beancoup simplifié le sujet de sa somrce:

- Pour la rédaction de Ferrari, v. Michele Caffi, Dull dbutzin di Chiuruvalle in Lomburdia. illustrazione

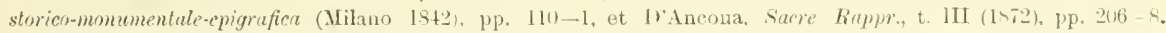
- Il est curieux de noter que, grace peutêtre i l'opuscule de Ferrari, la légende de santa froglielma s'etait localisée a Brunate: on racontait (en 184.j) que cetait lik que Gugliehna arait été retronvée par son mari. Dans l'église paroissiale de Brunate il y avait meme une lresque représentiut une scène de la vie de Guglielma et faisant partie d'nne série de peintures, alors détruites, relataut liflérents épisodes de l'histuire de

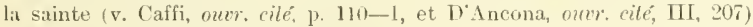

" P. 110, note. Cette analyse a été reproduite prar M. d'Ancona dans ses Sacro Rapprescntazioni, III (1872), 206-7. T)ans ce dernier ouvrage, 131, 208, on lit encore une seconde analyse de la version de Ferrari. trouvée dans les papiers de Pietro Monti, curé de Brunate, celui-là même qui, en 1s42, avait fourni à C'affi des renseignements sur le culte de Guglielma ì Brmnate.

${ }^{3}$ V. la 2se éd. (ls35), p. 143 (no. 92); (p. G. Passano, I Novellieri italiuni in prosu (Mlilano 1964), p. 245. ("est sans doute par erreur que le méme ouvrage a été mentionné dans G. Passino, I Vovelliri ituliun in verso (Bologna lis68), p. बरी.

* I. la bibliographie donnée par Colonb de Batines, Bibliogr. delle antikhe rappr. (175z, pp. 17-8 (reproduite par M. d'Ancona, sncr. rappr.. $111|1 \times i 2|$. 199-200). Dans le Dizionario di opere anomime o pseu

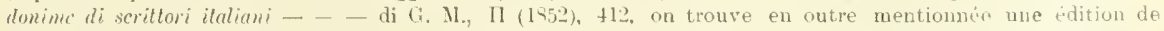
Florence de l'année 1551. Nous ponvons encore ajouter l'édition suivaute: Ln fista di sanlu liuglielma. Stanpata in Firenze Appresso Gionami laleni, VAnno MDLXXXV, in $4^{\circ}, 16$ jp.

s T. III, PP. $208-34$. 
Fipisodes: 1a) rest un seul rhevalier que le roi chargo de brîler Guglielma; l'episode (b) unauque: ciest quand Guglielma erre dans la forit qu la sainte Vierige lui apparait et que les deux anges vienuent

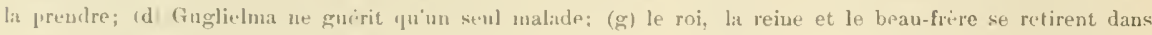
un ermitage'.

Nous ne samrions dire si mu ouvrage en . ottava rima“ de Fabio 'Tom(m)aselli.

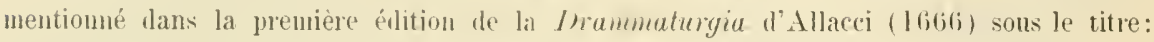

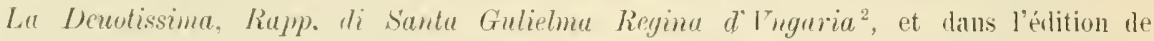
1755 sons les titres: Devolissima Ruppresentazione di Santa Gruglielma Regina d'Lngheria ${ }^{3}$, et Santa Gugliemu. Tita, P Miracoli di Srmta Guglielma. Fighola del he d'Inghilterra, "Moylie det her d'Ingheriu, con nltie cose utilissime ud oyni fedel C'ristiano", est autre chose quine reproduction de l'onvage d'Intonia Pulci. Un remaniement en prose du drame rersifié d'Antonia Pulci fut publié en 1720 (Venise, in-12. „per Domenico Lonisa") sons le titre: L'Imocenzu svelata in Suntu Guylielmu, ridotta in prosa per maggior faciliti da P. G. S. ${ }^{5}$ Ce remaniement, qui est livise en trois actes, a introdurt plusieurs noms proples et diffère en ontre par des détails le la Ruppresentuzione di Sunta Guglielmu:

Pirsmnages: (1) Alfonso; (3) Enrico. Épisodes: (a) un seul ange accompagne Guglielma juscuuau navire; (d) cest le capitaine méme du navire que Guglielma guerit: (g) lempire est donne à un chevalier, Belisario.

Un conte abmazien, publié par M. Antonio de Nino dans son ouvrage intitulé Usi e costumi abruazesi sous le titre Favola gentile ${ }^{6}$, omet l'épisode du meurtre, tont comme le drame de Santa Guglielma, mais. comme il raconte que le beau-frère est emprisonné dans la tom et qu'une fée indique à l'héroüne l'herbe merveilleuse, il faut bien regarder ce conte comme un dérivé populaire direct du mirate latin:

' lomme il n'y a qu'un criminel et malade, Mussafia (Wiener sitzungsber. LI, 6ljI - 3), qui ne connaissait pas les versions en prose, arait place la fiapresentazime dans son groupe l (ep. ci-dessus p 5). Or, les versions en prose appartiennent nettement au groupe 111 de Mussafia. Comme il est impossible de placer les versions en prose et le drame dans des groupes différents, on voit combien le systeme de classification le Nussatia peut etre arbitraire.

${ }^{2}$ V. Allacci, Diammaturgia (1666), p. 95: éditions de Nacerata (per Pietro Saluioni; s. 1. ?) et de Roneiglione (1619, per Lodouico Grignani).

3 r'ol. 249: éditions de Macerati et de Ronciglione 1619 (= celles mentionnees dans la première edition de la Imammalurgia d'Allacei).

('ol. 430: éditions de Ronciglione 1619 (v. les notes précédentes et de Padora s. d. (per (Fjo. Antonio Remondini).

s tette version fut signalfe par Fr. S. Quadrio, Molla storit, e della rayione dogni poesia. t. III (17t3), p. 11), ainsi que dans l'édition de 175.5 de la Trammaturgia d'Allacei, col 462 . Nous la conuaissons grace it un risume dritaillé qu"a bien roulu nous en faire M:lle Aline Pipping, dapres un exemplaire gue possede lis Biblioteca Vittorio Emanuele le Rome.

- Т. ПII (Iצs3), pp. 153-6 (no. XX17l, recueilli á Anversa, Bugnara, Cucullo, Sulmona, etc.). - M. lir. Ayrop (storia dellepoper francese nel medin ev\%, trad. Eg. Gorra |14.66], 1. 211, note 31 a le premier signali le rapjort existant entre le conte abruzzien et notre ligende. 
Yersonnages: (1) un mi; (2) Favola Gentile'; (t), (5) et (7) manquent lipisod's: (a) le roi, qui part jour un guerre. confie sa femme it la garde du frère; Farola Gentile cloit être jetée à la mer, mais les bourreaux (nles ministres") la placent sur un arbre au bord de la mer; l'arbre se brise, et Favola fientile tombe sur un rocher; l'épisode (b) manque; (d) sur le rocher oì était tombé Favola Gentile, une fée lui indiutue, pendant son sommeil, une herbe qui guérit tous les maux; (g) Farola Gentile reste auprés du mari et lui donne un fils.

Nous avous en maintes fois l'occasion de citer le beau mémoire de At. Mussatia. Über pine italienische metrische Darstellung der Crescentiasaye ${ }^{2}$. Or, cette version italiemue (vénitienne) de notre légende. intitulée Del duchu dTAngiò et de Costanza so mojer (en 67:3 „ottave“, 5384 vers), que Mussatia publia en grande partie, arec un résumé exact des parties non copiées ${ }^{3}$, provient le notre Miracle de la rierge, fort probablement par l'intermédiaire d'un roman français perdu ${ }^{4}$. La version italienne date

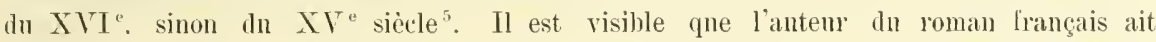
subi l'inflnence des nombreux romans d'aventure de son temps. En comparant la version Del ducha d'Anyiò avec le miracle latin, nons constatons les principales différences suivantes:

Personnages: (1) Lodovico, duc dinjou; (2) Costanza, fille du doge de Venise; (3) Clifet, nereu de Lodovico; (t) un comte; (5) Girardetto, neveu du comte; (7) manquent. Épisodis: (a) Glifet n'est pas emprisonne; c'est lui qui donne it quatre hommes l'ordre de tuer Costauza, arant le retour du duc; les bourreaux la laissent en vie, et elle entre en service chez une blanchisseuse; là, elle est recneillie par la femme du comte; (b) Girardetto étraugle l'enfant et accuse lui-même Costanza; celle ci est déposée sur une île déserte; (d) là, un ange lui tient compagnie et lui donme un vase, plein d'un onguent merreilleux; (e) c'est dans le

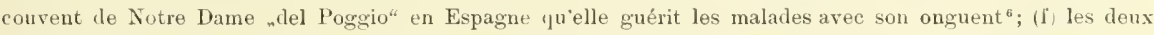
criminels, devenus léşreux, viennent, accompagnés de leurs oncles, au couvent où se trouve Costanza.

Il nons reste enfin, avant de quitter l'Italie, de mentionner une version popnlaire fort altérée, qui a été publiée en 1861, d'après un ms. de Bologne, dans l'ourrage intitulé: Sovelle d'incerti autori del secolo $\mathrm{II}^{+}$(t. I de la collection ścelta di curiositci letterarie inedite o rare dal secolo XIII al XIS)? ${ }^{7}$. Notre légentle y porte le titre: Storia duna doma tentata dal cognato, scumpata da pericoli, ritomata in grazia

${ }^{1}$ Nom singulier! Il semble bien qu'il y ait eu confusion avec lo titre général du conte („favola gentile", joli conte).

2 V. Sitzungsber. der philos.hist. Cl. der hais. Ahad. der ITiss. (Vieune), LI (année 1865), 589-692.

${ }^{3}$ V. ouvr. cité, pp. 598-6.59.

* V. Mussafia, ouvr. cité, p. 685.

${ }^{5} \mathrm{~T}$. Mussafia, ourr. cité, p. 590 .

${ }^{6}$ Dans LAmnalata, "commedia" de Giovammaria Cechi (1515-1587), laquelle n'est, d'ailleurs, en aucun rapport avec notre légende, il est raconté comment une femme, vivant cumme religieuse, guérit avec „un certo unguento", qu'une vieille femme lui a appris ì faire, son mari atteint de la lèpre, après qu'il a confessé son crime, qui ètait d'avoir voulu faire tuer sa femme faussement accusẻe (v. l'éd. Milanesi [1 556 ]. t. II, pp. 170-1 et 174). Peut-être qu'il y a là iufluence de quelque version italienne de notre légende. Du reste, l'idée de faire guérir un criminel par sa victime wême est tellement simple qu'elle a pu naitre spontauement chez différents auteurs. Ajoutons que M. H. Suchier, dans son édition des (Euvres poétiques de Philippe de Rími (t. I [18s4], P. LXVL), a renvoyé à divers contes populaires oì „le traitre (ou la traitresse) est. puni, comme le beau-frére de Crescentia, par une malarlie dont l'lèroïne seule est capable de le guérir."

7 Pp. 31-79. 
per sua castitu e diroziom. Cettr rersion prisente ceci de curienx que les deux premiers cipisurles, celui du hean-fròre ret celui rle l'assassin, sont combinés de telle manière que c’est le beall-frore qui tue l'entant de la belle-sanur. Toici les principanx maits caractéristiques de rette ression:

Personnages: 1) un homme demeurant it liome: (4) manque; (5) - (3): (7) manjuent. Fipisoles: it b) le mari, qui fait un royage d'affaires, laisse sa femme enceinte; celle-ci, lasse des poursuites du beaufrere, jrumet de se donner it lui apres avoir mis au monde son enlant'; comme elle ne veut pas tenir sa promesst, il tue son enfant "t lacruse Hle-meme de mavaises murs; le mari se resout alors it tuer sa femme lins un voyage, mais le eurur lui nanque, wt il se contente d'attacher sa femme a un arbre, apres yuni il contiuue sa route: (d) dins un songe, une adonna" dit à la femme de suive un lion qu'elle verra

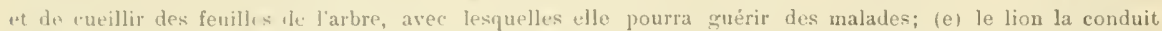
dans une ville? ou ell querit betancoup de maludes, et elle arrive enfin chez le comte "della llagna"; (f) lit virnnent le mari et le beaufrire milade: ( $g$ aprés la guérison du beaufrére, la femme et son ntari font bátir deux couvents, liun pour moines, l'autres pour nonnes, nú ils se retirent, laissant leur négoce entre les mains du frère.

Il a réjà été dit plus laut (p. 38) que le miracle de lo Tierge se retronse en lispagne sons forme d'mne tradurtion castillane de la version de Gautier de Coinci. Il est possible que loriginal immédiat de cette version es]agnole. me traluction galicienne: ait servi de base à la rersion galicienne qu'a donnce de notre miracle le roi de Caslille Alphonse X. surnommé mle Sage" (12.52-1254). dans ses Cántigas de Santa Maria. Dans la magnifique édition de ces r'ántigas, publiée en 1859 par l'Académie Royale d'Espigne, notre miracle porte le titre: Estu è como Santu Maria aindon a Emperalviz de homa u sofrelas yrandes coitas per que passou, et se compose de 26 couplets du 6 rers. munis d'mu refiain de 2 vers ${ }^{3}$. Cette version diffère par les traits suirants dn miracle latin (= la rersion de Gantier de Coinci):

I'ersmmages: (2) Beatriz; (4) un comte; (7) un marinier. Fipisods: 1a) il n'est pas dit que l'impératrice enferme son beau-frère par ruse: (d) une voix du ciel effraie les uarins.

Il se pent que la version galjoienne perdne ait aussi fommi le snjet d'me romance portugaise du XVI" siéele, qui a introduit partont des noms propres. C"est une

I'ne telle promesse se lit aussi dans la légende de Fuustinien, v. la Kaiserhronik (ėd. siclı̈̈rler). rve 131111314

[n lion serrant de compagnon et de défenseur est un trait assez conmuu dans la litterature

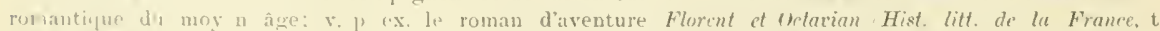

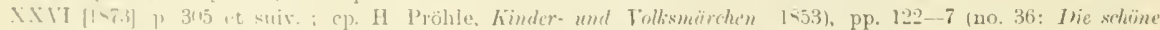
Yug alue). I. auni ci destons (j) sti) La version espagnole de Juan Jiguel del Fuego.

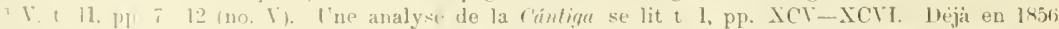

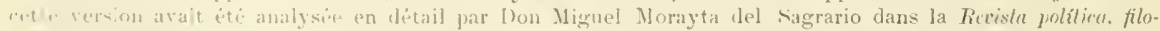

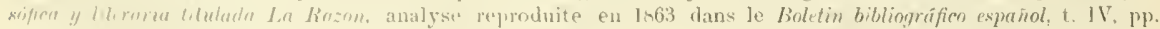

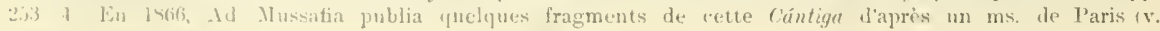

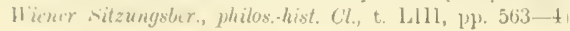


romance due a la plume de Balthasar Dias, natif de Malère, laquelle porte le titre: Historia da Imperatriz l'orcina, mulher do Imperador Lodonio de Loma, em qual se tratu como o dito Imperador mandou matur estu senhora, etc. Cette ronance a été publiée plusieurs fois comme fenille volante, et M. 'Th. Braga l'a reproduite, l'apres une édition de 1660, dans son ouvrage intitulé Floresta de rarios Romances (1s69)'. Cette romance se distingne par les traits suvants du miracle latin:

l'ersonnages: (1) Lodonio; (2) Porcina, fille du roi de Hongrie; (3) Albano; (4) le comte Clitineo; (5) Nathão. Eprisodes: (a) J'imperatrice nemploie pas de ruse pour enfermer le beau-frère; c'est it trois horrmes que l'empereur donne l'ordre de tuer sa femme; (d) l'imperatrice ne subit pas de vinlences de la part des marins; (e) la première personne qu'elle guérit est la femme d'un certain Alberto, chez qui elle ruste et aequiert sa réputation; (f-g) c'est li que viennent Clitaneo, avee sa femme Sophia, et Nathño; Clitanco et Sophia accompagnent Porcina à Rome; Sophia devient la "camereira de Porcina.

En espagnol notre miracle de la lierge se rencontre sous deux formes assez altérées.

An XVI ${ }^{e}$ siècle, Juan (de) 'Timoneda, libraire et antemr, en publia nue version dans son ouvrage El Putramelo (1'atraña 2), sans lui donner de titre². Cette vorsion a ceci de rmienx que l'autem y rent expliqner la trouvaille de l'herbe merveillense l'nne manière naturelle, sans l’intervention de la sainte Vierge. ('e n'est donc plus un miracle. Toici en quoi le récit de limoneda diffère du miracle latiu:

L'crsomages: (1) Narcelo, roi d'Angleterre; (2) Cieroneia; (3) Pompeo; (4) le marquis le (relia; (5) Fabricio; (7) manquent. Fipisodis: (a) les deux hommes, liobledo et Lobaton, qui ont reçu lordre de tuer (ieroncia dans la forêt commencent à se quereller au sujet de la possession de la reine; Robledo est tué, et le vainqueur est surpris frar le marruis de Delia; (b, d) la reine est conduite directement dans l'íle l)esalortunada pour y mourir de faim; lik, elte voit un jour comment une conlenvre, blessée par un lézard, place

1 Pp. 104-49. Outre l'édition de 1660, qu'a reproduite M. Braga, nous comnaissons des éditions de 1718 (Empratriz Porcina. Historia novamente de Emperalriz Porino mulher do Emperalor Lodonio di Toma. cm a quel se trata como o dito Emperador mantou matar a dita sienhora por testimunho, que lhe levantow o irmas an dito Emperador, e como escapou da morte, e dos muitos traballos, e fortunas, que persou, e de como por sua bondade, c muita limpeza tornou a cobrar seu estado crm mais homa, que a do principio. Lisbon occidental. Na Officina de Manoel Fernandes da Costa, Impressor do Santo Officio. In-4 ${ }^{\circ}$. $2+$ pl). de 1791 (Lisboa, por Francisco Borges de Sousa) et celle ipui fait partie de la Biblinthera pará o Povo (no. 26; v. Zs. f. roman. Thilol., IJl, fase. V [Bibliographie 1875], p. \$5); cp. I Fr. la silva, Dircionurie tibliogr. portuguez, t. 1 (1858), p. 322 (no. 11 ).

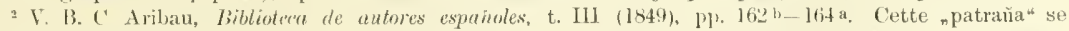
trouve dans les éditions d'Alealà, 1576 (v. Dunlop-Liebrecht, Geseh. der Irosudichtungen [1 551$]$. p. 5(k)h; Mad. den, Gresta Líminorum [1538], p. 522), et de Sevilla. 1583 (v. Grässe, Lelerbueh einer Literärgeseh. der berühmtesten Volker des Mittelalters. Wweite Abth., zwoite Ilälfte [1542]. p. 1032), lesquelles nous n'avons pas eu

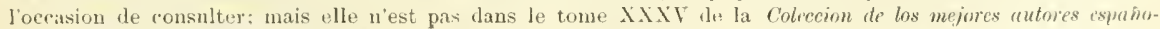
tes. qui contient El l'atrainclo. D'autres éditions du l'utranuch ont été signalées par F. Wolf, Jahbucher ler Literalur, t. CXXII (1845), p. 115. 


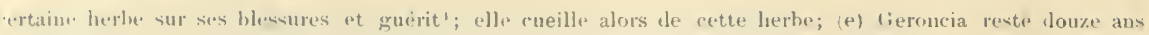

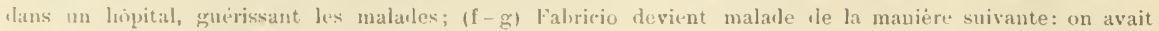
place l. "outeau ave lequel l'nfant avait the tue au-dessus de la porte de la ville, avee une inseription racontant le crime; or, le coutean tombe un beau jour sur Fabricio et le blesse grièvement à la tête; Geroncia ist, sur se's prieres. envoyie en Angleterre jar le marpuis de Delia; la maladie de Pompeo provient de ce qu'il a ité blessé dans un tournoi; avant de sa faire reconnaitre, fieroneia prie le roi llarcelo de la prenlre pour femme, co qu'jl reluse naturellement; le mari entre aussi daus un rouvent, et Pompeo recoit l'empire.

lantre version espagnole est me romance en lenx parties de Juan Mignèl del

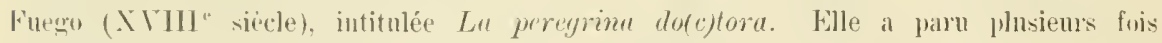
sums forme dle fenille volante ${ }^{2}$ et se tronve imprimée dans la biblioteca de autores espanoles l'Ariban ${ }^{3}$. ('ette rersion, qui a entièrement omis l'épisode dn menrtre, diflëre par les traits smivants do minacle latiu:

L'ersonnagis: (1) llon Alejandro de Figueroa y Sarmiento, grand seigneur de Lisbonne; (2) 1 toùa Ines Portorarrero; (3) Felerico; (4) (5) et (7) manquent. Épisodes: (a) le mari est parti pour une expédition le guerre: Doña Ines unferme Federico dans une maison quelle a fait bátir exprès: Jon Alejandro donne it quatre lommes lordre de tuer sa femme; les hommes commencent it se quereller dans la foret, et le combat linit par la mort de l'un deux; pendant la querelle la sainte Vierge apparait arec l'enfant Jésus et promet son sceours it lonia Ines; rolle-ci s’enluit, accompagnée d’un lion sue la sainte Vierge lui a enroyé comme protecteur, et trouve un refuge dans une grotte, oit le lion lui aphorte sa nourriture; les trois houreaux survivunt, fui doivent mpporter il lon Alejandro les yeux et le crur de Doña Ines, prennent les reux at le curur de leur eompanon tué ot les rapportent a leur maitre; a Federico ils racontent ceprendaut la verité; l'cderico va avec les exclaves chercher loña lnes; le lion tue les esclaves et blesse griévement feclerico, 'fui retourne aupris de son frère et lui raconte qu'un sauglier avait tue les hommes et

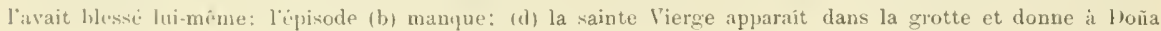
lıes un petit vase, plein diu baume merveilleux avee lequel elle pourra guérir les malades; (e) 1)oña Ines arrive i lisbonne, ou elle guérit tontes "jères de malarles; (f-g) la nouvelle de ces eures merveilleuses itant arrivic aux oreilles de Don Alejandro, également malade, il fait mander le célébre médecin, qui le guérit: puis elle gucrit le beau-frisre; elle reste ensuite aver son mari. et Federico éfrousu la sıenr de Holia Ines, Joña Elvira de tian I liego.

I Pour re trait assez commun dans le folk.Jore, ep. Ad. Ifussatia, II iener sitzungsber, philos,-hist. Cl., t.

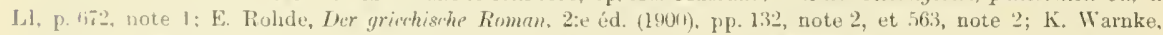

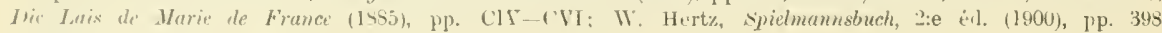
(.t tis-11 (note 1 ).

Nons connaissons les didions sujvantes:

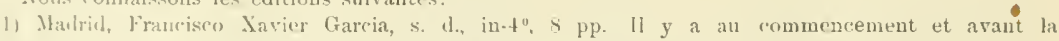
serconde partic um gravure refurésentant une femme eouche dius son lit, et à eote du lit, d'un róté, un -hevalier qui tient la main de la femme, de lautre, un diable cornu.

2 Vallarlolid, F. Santaren. \& I., in-4". \& IP. A commencement une gravure representant une fomme aver un baton le pielorin ì la main.

3) Baredona, Ignacio Estisill. s. d., in-to, s pp. Gravure au commeneement représentant une bereiere avee ses moutons

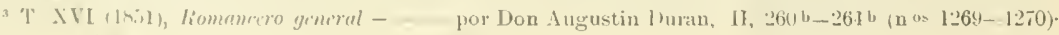

- Cp. ci-dessus l'une des versions italiennes (p. 5t). 


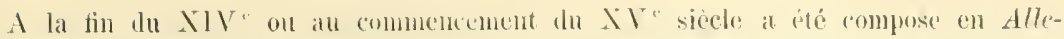
magne un livre religienx connu sous de nom de Der Seckn Trost' ef dont l'anteur' était peut-être un certain Johamnes Moirs sultze, de Cologne². Sous forme de dialogues entre nn maître et son élève. ce livre roulait. far des „exemples", illustrer les dix commandements de Dieu. Dans le lunitième rommandenent a aussi trouré place notre légende, peut-être diaprès la version de Vincent de Beaurais, que l'autenr lni-même dit aroir utilisé pour son aurre. La version primitive le Der Seelen Trost était jrobablement écrite en bas-allemand; anssi la plus anciemne édition comne, de 1474 , estelle en bas-allemand et s'ippelle leer selen troist. Notre miracle y porte le titre: Tan ezmer keyserymen de plach vnse leue vroure gerne ynnychlichen tzo eren ${ }^{1}$. De cette rédaction bas-allemande sont issues des versions en hant-alleuand, en hollandais. en snédois et en clanois. La première édition haut-allemande. Her sele troste, late de $1475^{5}$, et notre mirade y porte le titre: Ein keiser het ein fratren die dienet Maric". De la rédaction hollandaise on comait plusiems éditions à partir de 147s. Dans l"édjtion de $15\left(12^{7}\right.$ l'onrage est appelé Der sielen trowst et notre miracle y porte la rubrique: Hoe die keyserinne treemerf met loghen ter doot l'ordeelt was. end hoe se onse liene vrouce maria v'loste". La rédaction suédoise fut publiée par G. E. Klemming, d'après un ms. unique le la première moitié du XVe siècle, dans samingur af Svenstia. Fornskrift-Sällskupet (1S71-1873). Le titre en est Siäliuna Thrïst, ef notre mi-

1. T. pour la date de l'ourrage, G. E. Klemming, själens Tröst (18;1-3), p. IV.

2 l)ans l'exemplaire d'une édition, sans titre, date ni lieu. écrite en bas-allemand, "Iui se trouve dẹuis 1859 au Musée Britannique sous la cote 3834. b. 6 (le catalogue du Mus Brit. dit: "Ludwig ter Renchen: (ologne, $1455^{\text {? }}$ ), il y a sur un bout de papier, collé à un feuillet de garde, la petite notice suivante (en français): „L'auteur est Jean Moirs Sultze, de ('ologne qui visoit en 14tj. L'original a été rédigé primitivement en rers allemands." La dernière assertion étant sans aucun doute erronée, il est possible fu'il-ne faille pas non plus se fier à ce qui est dit dans la première phrase. Il est vraj que le r'atalogue du Iusée Britannique indique "Johann IIoirs comme lauteur de Ier Seclen Trosl, mais cette assertion peut étre simplement déduite de la notice manuscrite en question. Jean Moirs sultze était peut-ètre l'auteur de la tradurtion néerlandaise dont nous parlerons plus bas; c1. A. J. van der Aa, Biografisch Woordenboeh der Nederlunden, t. XVII (Haarlem 187t), p. 1017: "Van hem |siltzel is bekend Dir sielen troest Utrecht $1+79,--{ }^{4}$.

${ }^{3}$ Inprimée à Cologne, par Johannes Coilhoff.

4 Fol. $85 \mathrm{x}^{0}-86 \mathrm{x}^{0}$. Nous avons aussi vu des éditions bas-allemandes de 1485 (?, ('ologne?; fol.

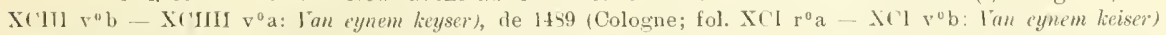

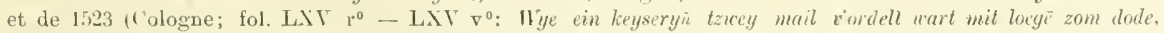
vn marin ir half). Une grande partie de la version bas-allemande de Iter sicelen Trost lut publiée, en $185+-$ 15.76, par Franz Pfeiffer dans les tomes 1. III de la resne The dutschen Mundartin (d'après un ms. de Stnttgart) comme une illustration du dialecte de ('ologne an XV:e siècle. Notre légenule s'y lit t. II, pp.

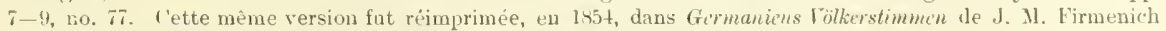
(t 111, pl). 212b-213b) sous le titre trompeur: Erzählung con der Crescentia.

3 Imprimée à Augsbourg.

"Fol. CXLI ro-CXLII $\mathrm{v}^{0}$.

7 Imprimée à Anvers.

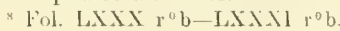




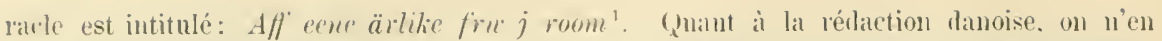

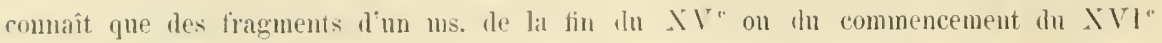
siecle couservés a Upsal et à Stockholm". T)ans le fragment de stockhohm (Kongl. Bibl.) notre miracle se lit sous le titre: Et miraculum uf jomfr muriue". La version de Der Seelen Trost ne diffère une très pen dn miracle latin:

Épisodes: (a) cest an Irère ilue lempereur confie l'empire et sa femme; r'est le bean frère qui fait arranger un appartement seeret dans la tour pour les rebdez-vons.

Assez près du miracle latiu est anssi lit „comédie“ the Kans Sachs intitulée: Ein comedi mit vierzehen personen, die unschuldiy keyserin ron Rom ${ }^{4}$ La pièce, qui a cinq actes ( $\$ 26$ vers), lite de l'année 15.il (31 aont) et est sans donte lat dramatisation d'm live: Kuiserin ron rom vertrieben (tradnction perbue du miracle latin) qu'on trouse mentionmé comme faisant partie de la bibliothèque dn célèbre dramaturge ${ }^{5}$. Si l'on excepte tontes sortes d'amplifications de détail, la version de Hans Sachs ne differe an miracle latin que par les traits survants:

Personnages: (3) Alphonsus; (4) le margrave de Salerne; (5) IIato; (7) un capitaine. Fipisodes: (a) le bean-frere raconte à l'empereur que l'impératrice l'a enfermé; il n'y a yu'un bourreau, qui ne cherche pas à faire violence à sa victime; le houreau fuit; (b) Hato place le conteau derrière le coussin de l'impératrice; elle est condamnée à etre noyée en mer; (d) le rapitaine du navire ne reut pas abuser delle; c'est un ange qui se montre a elle ${ }^{6}$ et place l'herbe dans son sein; (e-g) l'impératrice, habillée en homme, se rend directement à Rome. où viennent le margrave de Salerne et son frére lépreux; l'impératrice ne se lait pas nonne.

II nous reste à parlè d'une version allemande dh poète Hans Rosenbliit, de Nuremberg (milien dn XV siorlc), laquelle, tout en étant apparentée d'assez luès an miracle latin, présente ceci le curieux que l'emperem y est nommé Uctavianus, comme dans la version continentale des Gesta Romanom. On pent donc croire que Rosenbliit a conmu la version des Gestu Romunorum et que cest sous l'inflnence de cette version qu’il a aljelé l'empereur un miracle Octavianns. Ia version de Rosenblüt en vers plats. gui porte le titre: Ein liepleich history von groszer schone. gerhut und

1 T. Klemming, ouvr. cité pp. $\$+330$.

3 V. Klemming, ouvr. cité, p. VII. - I'our les diflérents mss. et éditions de cet ouvrage, r. J. Geffeken, Thr Bilderatechismus, 1 (15.55), 48 -9 et 111); Latendorf, dans Inzeigur fïr Kunde der deutsrhen

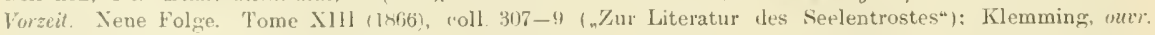
rité. pr. VI-VII

${ }^{3}$ Fol. $29 \mathrm{v}^{0}-33 \mathrm{r}^{0}$. Nous devons ee renseignement concernant la rersion danoise à l'obligeance d'une compatriote, M:lle J. af Forselles, doctenr ès lettres.

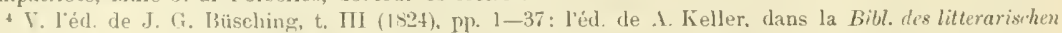
lereins in stuttgart, t. ('XXI (1sit: Hans vachs, t. VIII), 1P. 13I-61. Le prologue, contenant l'exposé de la piece, fut publić par H. F. Massmann, kaise'ehronit, t. III (18.34), p. 906.

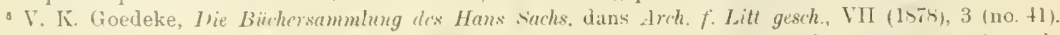
llans siachs.

- Irans rette altiration du miritele latin il faut sans doute voir un trait di an protestantisme de 
kuscheit einer edeln keyseryn, a été publiée par A. Keller, en 1853. d'après une vieille édition sans date conservée à 'Tubingne ${ }^{1}$. Il en existe aussi me traduction en vers has-allemands, éditée a Magdebourg en 1500 et qui porte le titre: Eyne schone hystorie van eynem keyszer to rome vade siner erliken Leyserinne wo de myt groter valseheit belagen wart². La version de Rosenblit diffère par les traits snivants du miracle littin:

Persomnages: (1) Octavianus; ( 7 ) un capitine. Épisodes: (a) c'est le frère cui reçoit le gouvernement de l'empire pendant l'absence de l'empereur: le lieu du rendez-vous est une tour sur laquelle on avait contume de faire mourir les malfaiteurs; le bean-frère s'y fait hisser secrètement, et l'impératrice se garle bien de ly suivre; le beau-frère accuse l'impératrice de l'avoir fait placer sur la tour: (di) Dieu ocenpe la place de la sainte Vierge; ( $f-g$ ) l’impératrice va directement à Rome, où elle gúrit son beau-lrère, ainsi que lassassin, qui y était aussi venu.

La version de Rosenblit a inspiré un "Meistersänger" du XVI" siècle, Albrecht Baumholtz (on Paumholtz), qui a écrit Die keiserin von fiom, poème de dix-sept strophes (de quinze vers chacune), jusqu'à présent inédit. C'ette rersion, que ırous ne comnaissons que d'après un seul ms. ${ }^{3}$, diffère de la rersion de Rosenbliit senlement par le trait snivant:

Épisode (d): cest la sainte rierge, et non Dieu lui-mème, qui apparait is limpératrice flormante. (Il y a donc, à ce quili semble, retour spontané it la version primitive).

En terminant ce compte rendu des différentes rersions du Mirucle de la ricrye. il convient de dire que la brillante réputation de la chaste impératrice de Rome a aussi trouré son expression dans nne ware diat figuré, datant prohablement du XIV* siècle, d'assez médiocre valeur du reste. Dom Bernard de Montfaucon, dans son onvrage intitulé L'Antiquité expliquee, et representée en figures (1719) ${ }^{4}$, a reproduit quatre tableanx sculptés en bas-reliefs sur les convertures en ivoire de deux tablettes à érrire, appartenant à l'abbaye Saint-Germain-des-Prés ${ }^{\circ}$. Ces quatre tableanx paraissent repré-

V. Keller, Fastnachtspieh aus dem fünfzchnten Jahrhundert. III, 1139_ 49. Tne autre édition sans date ni lieu, dont un extmplaire se trouve à Klosterneuburg (Allemagne), a été signale par J. M. Wagner dans le serapeum, XXII (1861), 62 ( Eim lieplich history von grosser schone, gedult vnd keuscheyt einer edleln keyseryn"). Pour les nss. de cette version, v. Keller, ouvr. cité, III, 132s (no. li): Fon der heyserin zu hom), 1431 (no. 3: Der keyser von Rom. Die keyserin von Rom) et 1433 (no. 1: Ihe heyserin von Rom Otuuianus weib).

2 T., pour cette édition bas-allemande, L. Götze, Aeltere fresch. der Buchlruclierkunst in Magdeburg, I (1872), 72-8 (no. 7), et C. Milchsark dans Arch. f: Lilteraturgisch., XI (1852), 164 -i0.

${ }^{3}$ als. Berlin, Cod. germ. 414, fol, $439 \mathrm{r}^{0}-442 \mathrm{r}^{0}$ (le ms. est un recueil de "Meistergesinge ${ }^{*}$, compilé par Hans Sachs). - Nous publions cette version dans notre Appendice (II).

+ T. III, partie II. pl. 'XCIV (à la page 3.56).

5 Ces tablettes sont actuellement disparues. 
senter denx lommes bafonés par des femmes (. Virgile dans la corbeille" i et "Aristote comme cheval" ") et leux femmes tidèles ("lat fille sans mains" ${ }^{3}$ et "L Limpératrice de Rome"). Sur le tahlean oì semble figurer notre héroïne, on roit, à gauche, une femme cueillant des herhes ot, a droite, un homme dans une maison aver les signes du léprenx, le chaperon sur la tête et liı cliquette à la main'.

\section{('HAPITIRE VII}

\section{Cirescentic}

Nous venous maintenant à un groupe de rersions où l'heroüne porte le nom de C'iescentia, nom quon a employé presque exclusivement, dans le monde savant, pou désigner, dans tonte son étendue, le conte de la femine chaste convoitée pur son beunfrere. Cor emploi alusif d'un nom qui nappartient de droit qu'à une très laible partie des versions de notre légende s'expliqute par le fait que c'est grâce à la présence d'une version de Crescentia daus la famense Kaiserchromik, publiée denx fois vers le milien dı XIX" siécle par des savants allemands, une fois par H. F. Massmann, l'autre par J. Diemer, que notre légende est entrée dans le domaine des légendes commes. Si à rela on ajonte que la version de Crescentia, telle qu'clle se lit dans la Kuiserchronik, remonte jusqua milien du XII" siçele, il est facile de comprendre l'importance „u'on a attribuée daus l'histoire de notre conte précisément à la version de crescentiu. Ft rependant, cette version n'est, selon tonte probabilité, qu’une variante du Mirucle de la Trierge quon a fait antrer dans une collection de contes quasi-historiques sur les emperems romains. Dans tons les ras, il est établi anjomrl'bui que notre légenle n’a pas fait pratie de lat source principale de la Kaiserchonik pour l'époque romaine. majs

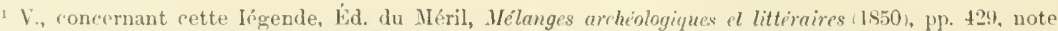
4, et 475, note 1; F. II. von der Hagen, Ciesommtabnleuer, t. III (1850), pp. 1 XXXIX (XILVI ( Der Zauberer

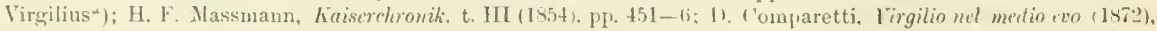
t. II, 11p. 105-1\%.

${ }^{2}$ V., concernant rette ligende, Ei. du Neril, Milanges arrkéologiqu's et littiraires (18.50), 1). 47t, note I; F. II. von der Ilagen, (iesammiabenteuer, t I (1850), pp. IXXT-LXXXII ("Aristoteles und Phyllis"); A.

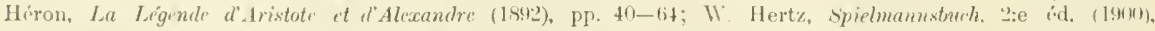
117. 24.3 52; A. Borgeld, Aristoteles e'n Plyllis (Groningue 1902).

${ }^{3}$ Pour cotte ligende, v. H. Suchier, Euvres poitiques de Philippe de Remi. Sire de Beaumanoir. t. 1 1KS4), pp. XXV-LXXIX

+1 p. II. Suchier, onur. eite. t I, pp. LIIl-LIT.

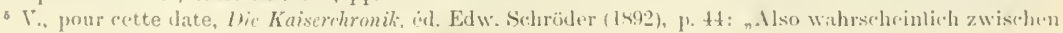
1147 und 115), kur\% gosagt um 1150 ist dic Kaiserchronik in Regensburg an lich getreten“. 
a été intercalée après coup ${ }^{1}$. Toici, d'après la version de la Kaiserchronik ${ }^{2}$, par quels traits la rersion de Crescentia diffère du miracle latin:

Personnages: (1) Nietrich, le laid; (2) Creseentia, fille d'un roi l'Alrigue; (3) Inietrich, le beau; ( $t$ ) un duc; (i) le śuéchal du duc; (7) manquent. Ípisodes: (a) introduction lans laquelle il est raconté comment les deux Dietrieh, fils jumeanx de Narcissus, suecesseur de son frère Hiraclius, et de sa femme Élisabeth ', tombent amoureux de ('rescentia"; elle choisit celui des frères qui était laiıls, et celui-ci derient par ce mariage empereur ${ }^{6}$; l'empereur part pour une guerre et laisse sa femme sons la garde du Irère; par suite de laceusation du beau-frère, Crescentia est jotée dans le Tibre, mais ello est sauvée par un jêcheur: (b) l'assassin place le corps de l'enfaut tué entre les bras de c'rescentia dormante; celle-ci est de noureau jetée dans le Tibre; (d) Creseentia est cette fois saurie par saint Pierre? ' 1]ui lui donne la faculté de guérir tous ceux qui confessent publiquement leurs fantes; l'épisode (e) manque; (f - g) ('resceutia se rend l'abord chez le duc, qu’elle guérit de la lépre, ainsi que le sénéchal; celui-ei est ensuite jeté dans le Tibre; accompagneje du duc, Crescentia ra à Rome, oủ elle guérit également de la lèpre son mari et son frère; l'empereur reconnait sa femme à une marque sur son corps; après avoir vécu ensemble quelque temps, l'empereur et l'imperatrice abandoment l'enupire an beau-frère et entrent au eouvent.

\section{La Kaiserchronit a été remaniée en prose, sous le titre Der künige buoch} Niuuer $\hat{e}$, vers $1275^{\star}$. Ce remaniement fut publié par H. F. Massmann dans le Land-

1. Die Kaiserchronili, èd. Sehröder, p. 58.

2 V. H. F. Massmann, Di'r keiser uml der kunige buoch oder die sogenannte haiserchronik, 11 1849), $146-24 \tau=\operatorname{rers~} 11367-12828$ (Narcissus; analyse détaillèe t. 111 [1854], 893-6); J. Diemer. Die Haiserchronik nach der ältesten Handsehrift des sitifles Vorau (1849), 1, 347-92; Edw. Sehröder, Hie haiserchroniti eines Re'gensburger Gistlichen (1892), pl. 284-314 = vers 11352-12812. In fragment de notre conte, correspondant aux vers 11411-11529 de l'éd. Massmann (éd. Schröder: vers 11395-11514), avait déjà été publié, en 1812, pąr K. T. Heinze dans Idunna und Hermorle. t. I, no. 36, pp. 143. 6 (ms. Neisse = Nassmann n, sehröder no. 211), et, en 181s, W. Grimm publia un autre fragment de la version de la kaiserehronik. correspondant aux vers 12197-12285 de l'éd. Massmann féd. Schröder: vers 12181-12269), dans la Leipziger Lileratur-Zeilung ("Zwoites Halbjahr“, coll. 13it-5; reproduit dans kleinere sihriften von Wilhcm Grimm. p. p. G. Hinrichs, II [1882]. -203-5\%. L'épisode de Crescentia a encore été reproduit, daprès la version de Mlassmann, par P. l'iper, lie spielmannsdichtung. II (1887), 221-58 (ouvrage formant le tome II de J. Kürschner, Deutsche Wationat-Litteratur). En 1853, O. Schacle essaya une sorte de reconstruction strophique en dialecte bas. rhẻnan de ce même épisode 204 couplets de six vers chacun) sous le titre: Crescentia. Ein niderrheinisches Credich aus dem zuölften Jahrlundert (p1). 69-110). Mentiomons enfin qu'il existe une médiocre traduction en prose allemande de la Kaiserehronik par J. M. Mayer (Der Kaiser und der Könige Burh oder die sogenannle Kaiserelronik. 1sït), oin l'épisode de Narcissus se lit pp. 199-219 (no. 29).

${ }^{3}$ Narcissus, Élisabeth et Ijetrich paraissent être des noms de fantaisie. Héraclius est sans doute lempereur d'Orient, le premier de te nom (610-641); ep. Kaiserchr, èd. Massmann, 111, 8xti- 7 .

+ Pour l'origine probable de ce nom, v, p. 62, note t. 11 est évident que notre ('reseentia n'est en aucun rapport avec sainte Crescentia, martyrisée sous Dioclétien: v, pour l'histoiro le cette sainte, Actu sanciorum. ed. nov., Junii tom. III (1867), ly. 491-519: „De sanctis martyribus siculis Vito, Modesto, ('rescentia" - (15 juin).

${ }^{5}$ "Der ungetane"; l'autre était appelé nder scône"

- D'aprés la loi de l'empire ( din $_{\text {phâhte }}$ ), relui des frères qui se marierait le premier serait enpereur.

7 Jacob Grimm (Gött. gel. Anz., annėe 1825, p. 707) regardait l'alparition de saint Pierre comme un trait plus ancien que l'aj'larition de la Vierge; de même llassmann (Kaiserchronik, IlI, $\$ 99$ et 905 ). A priori on est, en effet, tente de croire que l'introduction de la Vierge n'a eu lieu que postérieurement, sous l'influence du culte de plis en plus répandu de la Fierge. Quoi qu'il en soit, le Wiracle de la Vierge, en tant yu'étape dans le léveloppement historique de notre légende, représente un état évidemment plus primitif que la version de Crescentir. Pour ne mentionner que les traits les plus importants, cotte version non seule. ment a perdu toute notion du quatrieme criminel (le marin), mais elle a, en outre, inutilement puni de maladie le mari et le duc et introrluit un parallélisme fastidieux dans les deux scènes de guérison.

${ }^{3}$ V. Ihie helr, ed. Schröder, p. 76. 
und Lehenrechtbuch de A. r. Daniels. Liépisode the C'rescentiu n'y est pas ratconté en entier ${ }^{1}$.

Cet épisode se retronve ansi dans la Sächsische U'eltchronik, appelée également Replinusche (liepgonische) Chroniti, le plus ancirn omvage d'histoire allemande en prose (clatant probablement des annees 12:3-1251\%), qui a été publié ('n 1s: par Lulwig Weiland daus le seconl tome des Momumentu Germaniae historica, Deutsche

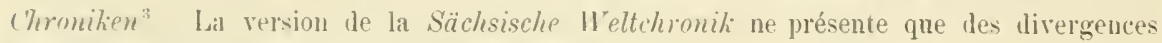
insignitiantes avec celle de la Kuiserchronik:

Persmuagis: (1) Dideric, le noir: (3) Diderir, le blanc. Fipisodes: (a) la tour est derrite fort minutien:ement et parte le $110 \mathrm{~m}$ de 'rispancia (variantes: ('ristancia, ('rescencia) ${ }^{\dagger}$ (g) le beau-frère entrc ansi alu courunts.

'Tout près de la version bas-allemande de la särhsische Welteloronik est une version en haut-allemand de notre conte, qui a été publiée, en 18:36. dans les dltdeutsche biätter de II. Hampt et H. Hoffmam sons le titre de Crescentia ${ }^{\circ}$. C'ette rersion ne differe que par les traits suivants de la version de la Sächsische Weltchronik:

Épisodis: (a) la tour s'appelle Engelborg; (d) c'est lange Gabriel qui rient en aide à ('rescentia, lorspu'elle ent jetée la seconde fois dans le Tibre ${ }^{7}$; lange lui dit de cueillir une herbe qui croit sous sa tête: cette herbe guérira tous les malades "jui confesseront leurs fantes".

Il a été dit plus hant (p. 60) que l'épisode de Crescentia n’a pas appartem an fond primitif de la haiserchronit. Il a donc dû exister comme conte ou chanson à

I. t I, fasc. T (156(1), pp. CL-('LI. Deux fois cette version renroie, pour des épisodes omis, à la Faistrchronik ( daz rint man an der Cronica*). Liempereur y est appelé nswarz llieterich".

2 V., pour cette date, silchsische Weltchronik. éd. I. Weiland (Won. Ferm. hist., Dinlsche Chroniken, t. 1] [1>7T]), p. 45 .

${ }^{3}$ P’. 139 \$3 (cap). 119). L'épisode ne se trouve que dans une partie des mss. de la sïchsische Weltrhonit. Aussi n'a-t-il pas éte publit par H. F. Massmann dans son édition de la chronique intitulee Ius Zeithuch de's Wike ron liepgou' (Stuttgart 1s5̃i).

- Dans la fioiserchronik cette tour ne portait pas de nom. Ici, il s'agit évidemment lu flâtéu saini-

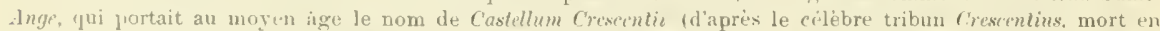

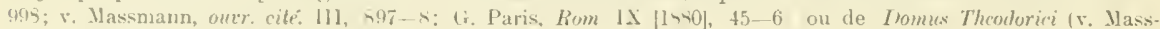

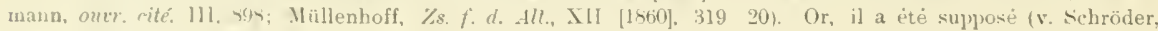
ouv rité. pp. (it et $29 !$, note 1), non sans vraisemblance, que lo nom de notre héroüne à été fait sur le non de la tour (cantellum Crescutii. Il se peut aussi que le nom de l'empereur et de son frère ait été inventé sous l'influence de lautre nom de la tour (domus Theodorici). En aucun cas n'est admissible la supposition de W. Wackeruagel (Zs. f. d. Alt.. VI [1st-], 156\%) qque la Domus Theodorici a tiré son nom du bean Dictrich de la ligende de crescentia.

"Dan deux des mss. de la sürhsische Niltrhonik, qui sont apparentes de pres (Dresde, Kol. Bibl. 154 d, et Hanorre, Kgl. J3ibl. Xl, 67t). cest Farchange Gabricl (et non saint Pierre) qui vient en aide i. Crescentia.

- T. 1. pl. 3(k) צ (l'après le ms Lejpzig, Bihl. Univ, no. 1979, XV:e siècle). lne réimpression

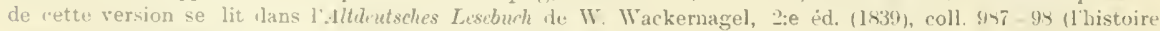
116. st: troure pas encore dans la litre edition, de 14.3\%.

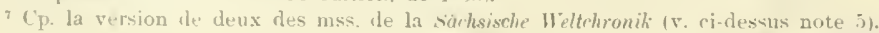

- A cet endroit il $\mathrm{y}$ a cividemment influence directe diune version du Miracte de la Viergc. 
part dans la première moitié dn XII siècle. U'est pent-être un dérivé de cette rorsion primitive que nous avons dans me chanson en vers plats de huit syllabes (environ losu vers), conservée dans quelunes miss. du XIVe siècle, si l'on ne prélère regarder cotte chanson comme détachée postérieurement de la Kaiserchronik ${ }^{1}$. Dans tons les cas, les deux versions sont apparentées de très près; sourent même les vers sont presque identiques ${ }^{2}$. Cette version de l'histoire de Crescentia fut publiée en 1 Sit, d'après le ms. lit de Kolocza, par le Comte J. N. Mailíth et J. P. Köftinger dans leur ourrage intitulé Koloczaer Codex altdeutscher Gedichte ${ }^{3}$, et, en 1850, F. H. von der Hacren jmblia la. même version, d'après un ms. de Heidelberg (Cod. palat. 341), dans ses Gesummtubentener sous le titre de Crescrntia ${ }^{4}$. Ontre quelques analyses en prose du conte ${ }^{5}$, il fant. encore mentionner deux modemisations en allemand, l'une en vers par le comte d. N. Mailáth $(1819)^{6}$, l'antre en prose par J. P. Lyser (1S38) ${ }^{7}$. - Cette version, que nous appellerons la Charrson de crescentia, ne diffère que par quelques traits insignifiants le la version de la Kaiserchronit:

Épisorles: (a) c'est le pape yui déciele que celui qui se mariera le premier aura l'empires ${ }^{8}$ (g) Crescentia et sen mari ne restent ensemble qu'une semaine.

La version de Crescentia nous a encore été conservée dinns deux livres populuires.

Limn de ces livres populaires, que nous comnaissons dans une réimpression moderne de O. F. H. Schönhuth, portant le titre: Historie oon der gedluldigen Königin Crescentia", rappelle par plusieurs traits la version des Altdentsche Blätter, mais semble en même temps être basé sur la Chanson de crescentia. Nous annotons ici les divergences avec cette dernière version:

Personnages: $(1$ ) et $(3)=$ Alld. Bll. Fipisodes: (a) la tour est dicrite comme dans les Altd. Blitter; (d) c'est un ange 'qui vient en aide à C'rescentia et lui indique l'lerbe sous sa tète (c). les Alld. Bll.) io.

1 En géneral, on a admis cette seconde alteruative. La première alternative it ité defendue par $K$.

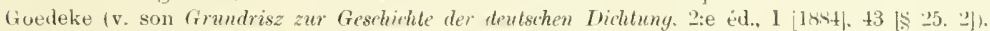

2 F. H. von der Hagen (irsammlabenteuer. I |1850|, 513--21) a comprà les vers que cette version, d'un côté, et deux des mss. de la kiaiscrhronik (Heidelberg, cod. pal. 361 - Massmann H, schröder no. t, et Tienne, K. K. Hef- und Staatsbibl. no. ¿693 -. Massmann W, schröder no. 16), de l'autre, ont en commun.

${ }^{3}$ V. pp. 245-7t (Aventure 48). Liannée suisante (151-). les vers 599-6ss furent réimprimes, aser des corrections, dins un compte rendu de louvrage par IV. Grimm daus la Leipziger Lileratur-Zcilung. "2-e

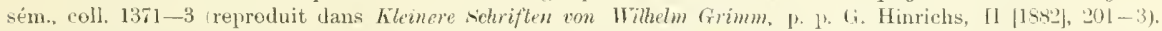

* T 1, pp. 135-6+ (no. 51 du ms,). Une analyse lu poéme est domée pp 131-3.

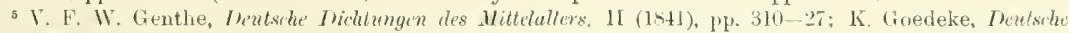
Dirlutung im Wittlatter (1854), p, 160 b-161 b Goedeke traite on meme temps de la version de la liaiscrehronik.

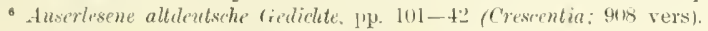

7 Abendländische 'Thusend und rine Nacht, V, 15l-s.'

* II nous semble probable gue la chanson de crescentia présente jei une alteration le la leşon primitive, Toumie par la Kaiserchronik: diu phâhte a tite interpreti comme der babrst (le pape)

"Rentlinger, s. 4, in-12., 32 Ir). Le livre est divise en neuf ehapitres. Sur lo lenillet du titre il $y$ a une gravure représentant un ange à genoux auprès de l'rescentia dormante.

io $11 \mathrm{y}$ a encore $u$ petit ditail qui montre literite altiniti, pour certains passages, entre eette

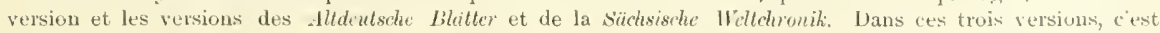


lautre live pmonlaile. yni nous est comm par une édition du XVI" siède. porte le titre: Ein sehone und wahruaftige hystori von einer Kayserin zu Rom: genunlt Corscentia: gar lurtzaryliy zulesen'. Cutte version, qui présente certaines altérations dues à luntluence do versions étrangèress à la version de Crescentia, diffère par les traits suivants de la Chanson de Crescontia, de laquelle elle se rapproche le plus:

I'rsemnages: (2) rescentia, fille de lempereur de Rrsme, Octavianus: Fpisodes: (a) les deux l)etrich ne sont que princes romains; c'est la sainte Vierge qui ronduit ('rescentia à la maison du pécheur; (b) le sénechal est le propre frère du (luc ${ }^{3}$; le meurtre s'uccomjlit en l'absenee de ('rescentia; (al) la sainte Vierge save crescentia et lui done unc herbe pour guérir les lépreux*: (e) Crescentia guerit plusieurs persomes, avant daller chez le duc ${ }^{5} ;(f-g)$ le simeloal ext exile; ce sont des messagers de l'empereur qui conduisent Crescentia à Rome.

Il nous reste entin à mentionner la rersion métrique fortement abrégée, basée probablement sur la Chunson de Crescentia, qu'a léguće à la postérité le poète didactiyue autrichien de la seconle moitié du XIVe siocle Heinrich der Teirhmer ${ }^{6}$. Cette rersion, inélite jusquà prisent, diffère par les traits suivants de la Chanson de Crescentia ${ }^{7}$ :

Persomngex: (1 3 i sans noms. Episodes: (a) l'introduction manyue; (f-g) le duc ne devient pas malarle; l'impiratrice guérit les malukles, au noyen d'un reméde, dans l'ordre suivant: le mar'i, le beau-frère, It. senechal; les deux derniers sont jetes dans le Tibre; les epoux rirent ensemble deux annees.

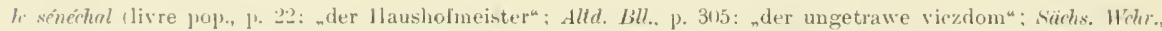
p. 14?: nte untruwe vimedom") qui conseille le premier de noyer Crescentia; dans la version de la Kaiserchronit et lans la ('honson de Cresention, ce conseil est donné par la femme du dur (hichr., éd. Schröder, v.

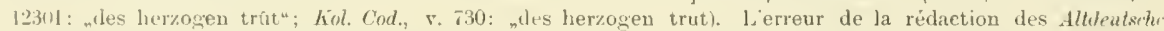
Blithr ut fle eclle de la sïchsische IV dthonik s'explique par le double sens du mot trüt (npersonne en qui on peut se fier, ronfident, ami" et, plü speicialement, femme, épousen). Nous sommes surpris que $\mathrm{M}$

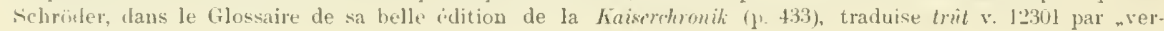
trauter". 11 nous semble de toute evidence, a cause dn $r$. 12306 (wer solte sich an wibe rede cheren?" ıu'il sagit de la femme du dur. (1). L. Weiland, ouvr. rite, p. 142, note 2.

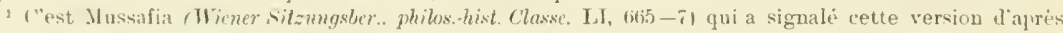
une idition in.$^{\circ}$ sans date, imprimé a Landshut, dont un exemplaire se trouve a la Bibl. imper. de Vienne. M:Jles IJedvig et lrma Rosen, de Vicnne, ont bien roulu nous fournir une cojie fle cet exemplaire.

2 11 y i sins doute. à cause de ce nom, guelque reminiscence de la version continentile des Gesia Rommorum (v. p. 2⿰氵).

3 Influence du Wirate de ln Vierge?

tp. la version du lirre jropulaire public par sehönluth.

s ('p. Je Mirarle de la Vierge (ri-dessiss p. 34).

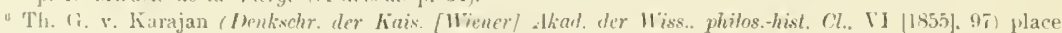

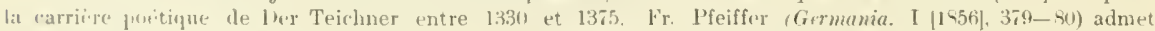
"romme soules dates sures les années 1350-1377. - Le premier vers du prème: "In der Römer puch man las" fumrait faire supjoser que le prieme est tire directement de la haiscrdironik, mais probablement il ne faut y voir quine de ces indications trmpreuses destineies it rassurer des lecteurs sceptipues. Le contenu du preme de Iner Teichner laisse la question ouverte.

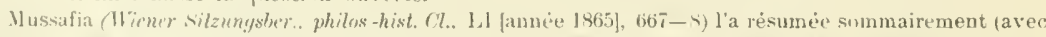

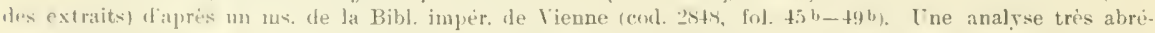

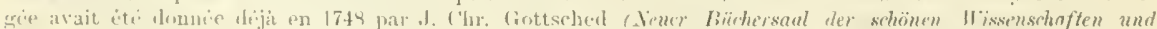

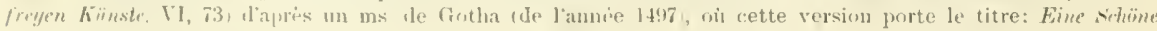

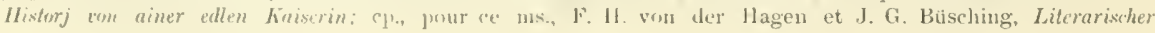




\section{CHAPITRE VIII}

\section{Hildegarde}

Jusqu'ì présent, notre légende n'a eté rattachée qu'à les persomnages fietifs ou vaguement définis. Avec le grompe de versions que nous abordons maintenant, nous entrons en pleine histon'e: l'héröne, Hildegarde, est la deuxième (ou troisième) femme de Charlemagne (morte le 30 avril 783 ), et le mari, e'est Chirlemagne lui-même.

La plus ancienne de tontes les versions d'Hildegarde est une version allemande mammcrite, faisant partie d'une chronique de l'abbaye de Kempten en Baviòre. écrite en 1507 de la main de Johamnes Kräłler, notaire de Kempten, et appartenant anjourd'hni ¿i un particulier de cette ville ${ }^{1}$. Cette version, qui a été publiée par M. Kar lieiser lans ses Sagen, Gebräuche \& Sprichü̈rter des Allyäus, aus dem Hunde des Volkes gesammelt. tome I (1897), sons le titre: Hildegard und Taland ${ }^{2}$, se tronve anssi dans qmelques antres mss., postérieurs à celui écrit par Kräler: Liautenr en fut fort probablement mu certain maître d'école, Johannes Birckins (Birck), yni a composé diverses chroniques mensongères sur labbaye de Kempten. La chronique que nous possédons dans la copie de Kräler, chronique continnée par celni-ci jusqu'en 1507. ne pent avoir été écrite qu'après 1481, probablement en 1484 on 1455'. Dans cette chronique Birck a roulu ajouter un nouveau trait à l'homueur de la patronne du couvent de Kempten en mettant sur son compte une piense légende quil comaissait. Vu la manière extrêmement légère dont Birck traite la vérité historique, il est très difficile de dire si Birck a entemuln

Crrundriss zur Geschichte der deulschen Poesie (1812), pp. 409-10; Fr. Jacobs et F. A. Ukert, Beilräge zur ältern Litteratur. 11 (1836-1837), 316-7. Une copie, faite par Gottsched. de la version du ms de Gotha se trouve dans la Bibl. royale le Tresde (M 203); c'est la copie signalée par Fr. Adelung, Altdeutsche Greliehte in lirm ete. (1799), pl. XXVIII-XXIX (Churfürst1. Bibl. zu Dresten Nr. 105). - Nous publions la vursion te ller Teichner dans notre Appendice (N) d'après la copie de ('rottsched.

1 M. Martin Leichtle, rentier, qui a bien voulu nous permettre de prendre connaissance du ms. en ipuestion ‘,Kleinere Kräler'sche Chronik“, fol. $23 \mathrm{r}^{\circ}-28 \mathrm{r}^{\circ}$ ).

${ }^{2}$ Pp. 44?-S (no. 54:?

3 Nous avons vu cette meme version, avec des variantes de peu dimportance, dans les mss. Munich, Kgl. IInf- und Staatsbibl.. end germ 5821 ( $($ homnik des Stiftes u der Grafschalt liempten, zusammeugetragen von des Stiftes Holmeister Georg Fläschütz 15+4"), fnl. $5+r^{0}-5 \checkmark r^{\circ}$ (la fim, après le passage on il est dit qu'Hildegarde bàtit le couvent, manque), et col. germ. 582’ (copje du XVII:e siècle d'une partie du ms. précite), fol. o $\mathrm{r}^{\circ}-16 \mathrm{r} \mathrm{r}^{\circ}$ (sans pagination).

+ Pour ce qui concerne les „Lügenchroniken ${ }^{L}$ de Kempten et leur auteur presumable, v. F. 1. Pinumann dans Alemannia. Zeilschrift fïr Sprache, Litleratur und Volkskunde des Elsasses, Oberrheins und

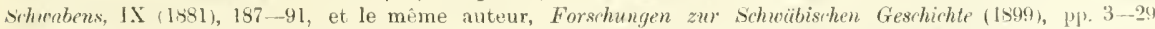
fonmme extrait de cet nuvrage avait paru déjà en 189.5, lans la revue Allgäuer Feschichtsfreund. anmée 1895, p. 61 et suiv. un mémoire intitule: Die hrmptner Chronikon des ausghenden 15. Jahrhuudorts). Dans le ler-

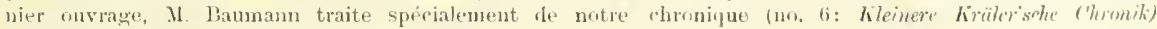
a11. 11. $14-23$. 


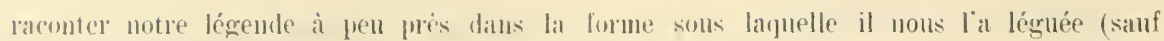
les noms propres ef li fin concerninn le courent de liempten). on bien sil a lihrement arrangé nne version du Mirucle de la Tirrye, qui est daus tons les cas la source, soit directe. soit indirecte, de la rersion de Birck'. Nons sommes très porté à corre que la seconde alternative est la home, rar, dune part. nons ne commissons ancme version de notre légende yui soit à pen pròs semblable à celle de Birck, moins les noms propres et la fin: d'autue part. il est assez naturel que Birck, pour ne pas s'exposer trop facilement à ĉtre afensé de plagiat et de mensonge, ait altéré la version qüil avait devant lui un qüil arait entendu raconter. Voici far quels traits la version de Birck (copje Kräler) diffère dn mirale latin:

Prromnags: (1) ('harlenagne; (2) IJillegarde; (3) Tialanul: (4) le chevalier de Freydenberg, au service In la sreur d'Hildegarde, Adelinde; (5) it (i) manquent. Fisodes: (a) Charlemagne, partant, en i65, pour rombattre les infideles, conlie llilelegarde et ses enfants a Talaud; celui-ci construit un palais avee trois portes. l'une derriere l'autre, pour les rende\% vous: il raunte ì Clarlemagne qu'Hildegarde l'avait enferme;

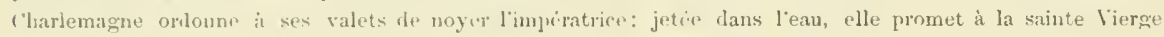
de hatir un couvent, si elle ichaple il la mort; saurée, tlle parrient it une maison princière. oi elle est bit-n traitie; un jour qu'elle se tenait it une l'nètre du chatean, llle est découverte par 'Taland et charle. macrne, uni passaient jrés de lá; 'harlemagne ordonue cette fois à ses valets de la conduire dans une foret t-t he lui arracher les yeux; le chevalier sauveur, qui connait Hildegarle, laisse les valets, qui nont pas soulu violer leur victime, prendre les yeux do son chien pour les remettre á l"empereur en signe de leur obcissance; les ajpisodes (b) et (d) manyuent; (e) Hildegarde s'expatrie, en compaguie d'uue demoiselle Rosina de Bodmann, et se met i itudier les qualites curatives des plantes et des pierres précieuses, de sorte "juelle arrive à guérir toutes sortes de maladies, surtout la recitri; sa réputation devient si grande, yu'elle est appelée par le pape Lérn it Rome, ou elle continue, inconnue, d'exercer son métier de mélecin; (l) Taland, devenu lépreux et avengle, se renil avec l'empereur i Rone pour consulter la célébre femune; (g) quand il arrive a la inaison d'Hildegarde, celle ci lui fait dire par son anie yuil doit dabord aller se confesser; comme il ne dit pas son crime envers llildegarde, il est renroyé se confesser; étonnés de la guérison 1. Taland. le pape et lempereur veulent savoir qui la femme est: elle apparait à l'église Saint-Pierre et se fait connaitre; Taland est exile; pour tenir sa promesse. Hildegarde lait hátir le couvent de kẹpten et le drite de riclies domaines.

En lisant la version de Birck, on se demande comment Birck a en l'idée d'attribuer as la patronne de l'abbaye de Kempten le sort qu'amait subi une impératrice de Rome non nommée. Fist-ce quil y avait en, par hasard. quelque ressemblance entre lat vie l'Hildegarde et celle de l'impératrice du miracle latiu? Nous avons consulté les chroniques do moyen âge alin de siroir si Charlemagne a répudié Hildegarde. alle

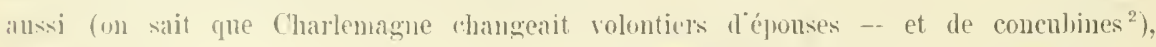
mais en vain. Ce quon sait d'authentique snr la vie d'Hildegarde, se réduit à peu

\footnotetext{
'Lassertion de Birck, au commeneement de sa version de notre légende, qu'il l'a tirée ,ron alter poetischer latin ${ }^{4}$ est naturellement sujette i cuntion.

${ }^{3}$ Thans la version même de Birck nous trouvons un enrieux témoignage des mours peu rigides du yraut monargue. Lat chronique dit que, quand l'empereur ent retrouré son épouse, no tett der selbig kaicer Kamblus die köjs\%wyber alle von im" (id. Reiser, p. t4i).
} 
près anx faits suivants: Hildegarde, fille d'Imma. arrière-petite-fille de Godefroy, duc d'Allemagne $(\div$ en 709$)$, naquit en Somabe ${ }^{1}$ rers $757-758^{2}$; à l'âge de douze ans accomplis, pendant la seconde moitié de l'année 770 on an commencenent de l'année ið, elle devint l'éponse de Charlemagne ${ }^{3}$, après que celui-ci ent répudié lis fille de Didier, roi de Lombardie ${ }^{4}$, elle donna a son mari quatre fils: Charles (f en s11).

1 Einhardi Tita Karoli Imperatoris, cap. 14 (Mon. Germ. hist., script, tom. II [1599], p. 453): „Hildegardem de gente Suavorum, praecipuae nobilitatis feninam, in matrimonium accepit; ${ }^{*}$ Thegani Vita IIlulowici Imperatoris. cap. 2 (Won. (rem. hist. script. tom. II [1829], pp. 590-1): „nobilissimi generis suasorum puellam, nomine Hlildigardam, yuae erat de eognatione Gotefridi ducis Alamannorum. Gotefridus dux genuit Huochingum, Huochingus genuit Nebi; Nebe gentrit Immam; Imma vero genuit Hiltigarlam beatissimam reginam". l hans les „ehroniques de Kempten" il est raconté "lu'Hildegarde était fille dHildebrand, duc de Souabe, et de la Bavaroise Regarda, qui résilaient au chateau d'Andechs en Bariere, assertion tenue pour

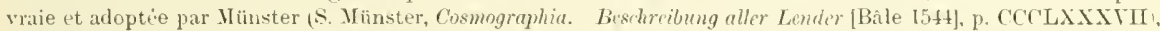
Brused (G. Bruschius, Chronologia Monasteriorum Germaniae [éd. 1692], p. \$9), Rader (II. Rader, Bavaria Santa [1615-162t], t. 11. p. 103) et nombre dautres listoriographes des XVI:e et XVII:e siécles. (\}ue le nom de la mére l'Hildegarle ait été „Imma", c'est ce qui ressort aussi d'une charte de l'année 786 , provenant du frère dHildegarde. Gerold, dans laquelle on trouve parmi les témoins: "Imma genetrix" (v. H. Wartmann, Urkundenbuch der Abtei sanet Gallen, I [1563], 101-2, no, 108; op. aussi Chr. Fr. Stalin, Wirtemberyisehe Greschichte; 1 [15+1], 245).

" C'ette date n'est pas indiquée dans les chroniques du moyen age; mais, comme on sait "lu'Hildegarde est morte le 30 avril $\mathbf{7} \mathbf{3}$, elle peut ètre déduite d'un passage de l'épitaphe bien connue composée par Paul [racre a loccasion de la mort de la reine (v. Pauli WarneTrdi Liber de Episcupis Motlensibus dans les Mon. Germ. hist.. seript, tom. II [1529], p. 266; ep. Mon. Germ. hist. Foetae latini aevi Carolini, t. I, part. I [15-0]. Pp. 55-9: Epitaphium Hildegardis Riginae, vers 21-24):

- Alter $a b$ undecimo iau te susceperat annus,

Cum ros mellifluus consotiavit amor;

Alter $a b$ undeeimo rursum te sustulit annus,

Heu genitrix regum, heu decus atque dolor!

Par „alter ab undecimo" Paul Diacre a sans donte roulu dire .le treizième". quoique Virgile semble aroir employé la mème expression dans le sens de ndouzième" (Ecl. VIII, 39: „Aller ab undecimo tum ne iam ceperat annus"); pp. J. Havet dans la Fibl. de like. les Chartes, t. XLVIII [19si], p. 4!. Mais déjà le grammairien Servius (IV:e siécle) expliquait l'expression de Virgile par ,tertius decinus" (v. Commentarii in Tirgilum Serviani, ed. H, A. Lion [1526]. II, 151), et les denx commentatemrs de Térence, Donat (IV:e siècle)

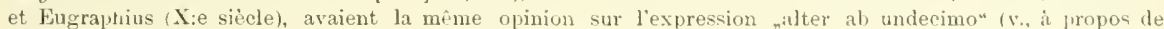
Andria I, I, 51, l'éd. de R. Klotz, t. I [1838], pp. 2l et 178-9). - L'assertion de Brusch qu'Hildegarde serait née en 732 (v. G. Bruschins, oucr rité, p. 91) est toute fantaisiste. De méme, il n'y a aucune nreuve sérieuse pour l'année 753, proposée par D. Papebroch (v. Acta simutorum, A pr. t. ПI [1675], j. 7!) [Comm. ]raev. cip. 13]).

${ }^{3}$ En laveur de lis première date parle une charte du mois de juillet $\%$ i, dans lacuelle Charlemagne mentionne dejà son fils Charles, l'ainé de ses fils avec Hildegarde (v. cutte charte dans la Bibl. de l'Ée. des Chartes, XLVIII [1857], 226-8, et les commentaires de J. Havet, ibirl., pp. 46-8); cp. cependant, sur la valeur de eette charte, S. Abel et B. Simson, Jahbïcher des Frünkischen litiches unter Iran dem Grossen. I (ä:e érl., 1848), 673. Jlans tne autre charte, probablement aussi apocryple, du 1:er wai 793 ( $v$. S. Abel et B. Simson.

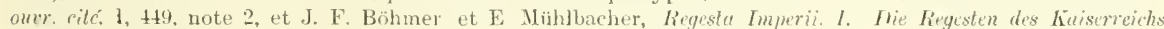
unter den Karolingern 551-918. I [1589], 95 [no. 253]), Charlemagne dit avoir pertu sa lemme dans la treizièm année de leur mariage ${ }_{n}$ dulcissima coniux nostra obiit in ano tertio decimo coniunctionis nostrae": v., sm." l'authenticité de cette datation, J. Havet, Bith. de l'Ée. des Chartes, XLIIII [Iss]], 50, note 1). Encore un

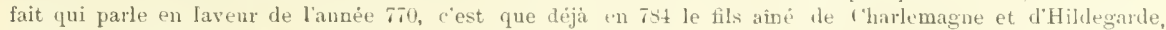
Charles, remportait une victoire sul les Saxons (x. S. Abel et B. Simson, ou'r, rite, I, 4ris-5; cls. J. llavet, arl cité. p. 51).

+ V. Einhardi lita Liaroli Imperatoris, eap ls Von. Gierm. hist., seript. tom. II [1 529$]$, 1) 453): „Deinde cam matris hortatu [Karolus] filiam Desiderii, regis langobardorum, duxisset wxoren, incertum gua de causa, prost annum eam repudiavit, et liildegardem _ _- in matrimonium accepit"; "l). Monuch. Sicmyall. II, 17 (Mon. Germ. hist., seript. t. II [1829], p. 759): „filian I)esiderii Lungobardorum principis duxit 


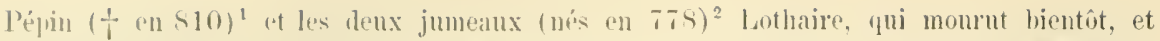
Lonis, qui desint le surensem de charlemagne, ainsi que cinn filles: Rothrule, Adélaïde.

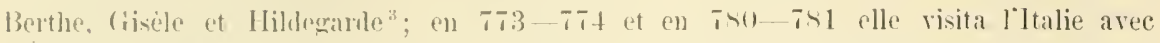

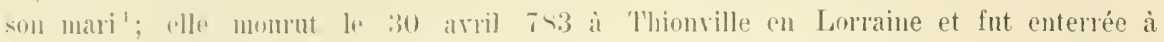

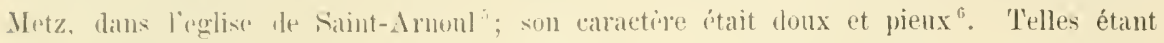

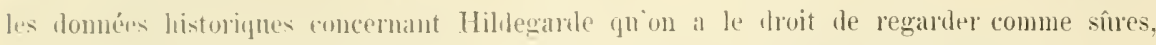
il va sans dire que les lypotheses de quelques savants, qu'Hildegarde aurait été la premirra femme de Charlemagne, abandomée pour la fille de Didier et puis reprise après

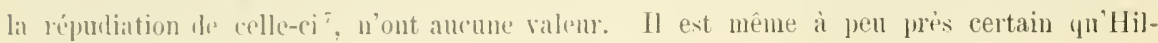

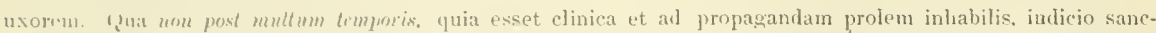
tiscimorum sarerelotum relieta velut nortua, -. *. 11 est fort probable qu'Eginhard sest tronipé sur la

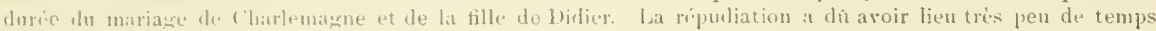

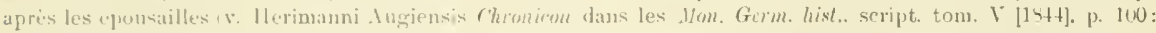

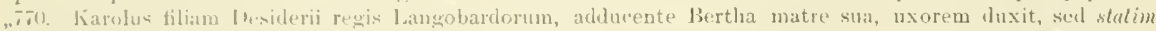

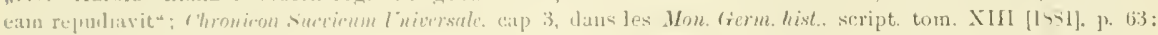

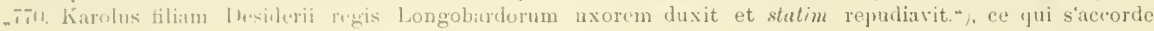
aver fe yni a iti dit dans la note préridente sur la date du mariage de /'harlemagne avec Hildegarile.

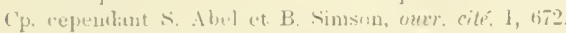

1 I., pour la dite de la mort de ces deux dils, Thegani lita Hudourej Lmpcratoris. cap. 5 (Mon.

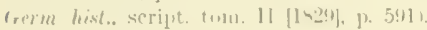

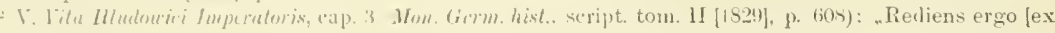
lli-panial rex mplerit coningen lliliegardan hinam edidise prolem masculam; quorum unns inmatura morte prareptus, ante pene nori quam sub luce vivere coepit, alter [Hludowicus] prospero eventu materno fusus ntem, infautilibus nutriebatur inpendis. Nati sunt antem anno incarnationis domini nostri lesu ('hristi septimgentesimo spptuagesimo ortaro".

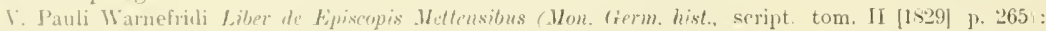
.ex Hihlegard coninge quattuor filios et quinque fllias procrearit". Einhard nomme trois filles (v: Einhardi

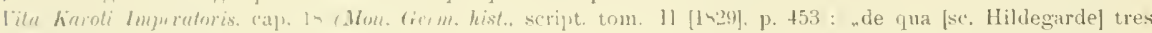
tilios, kitrolum videlicet et J'ippinum et Ludowicum, tothemgut filias. Hruocindem et Bertham et Gislam,

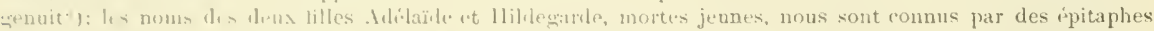

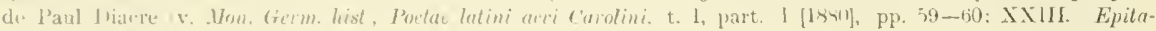

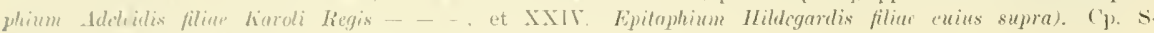

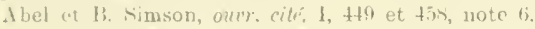

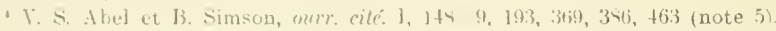

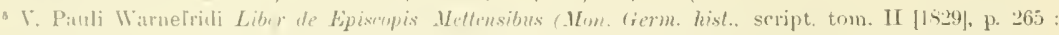

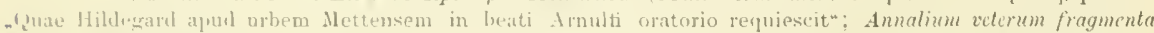

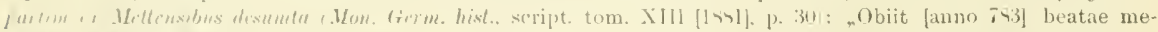

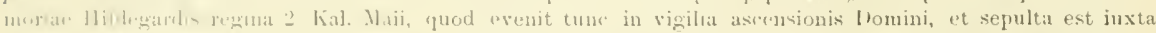

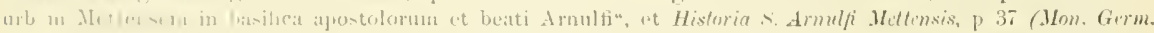

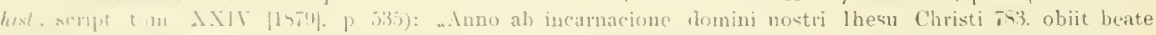

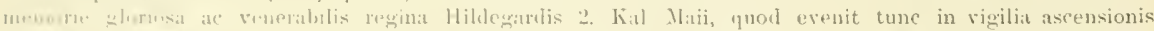
1нum

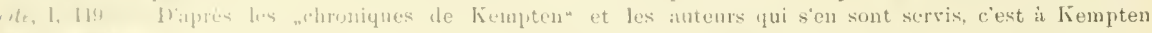

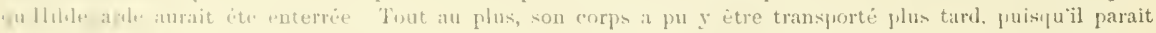

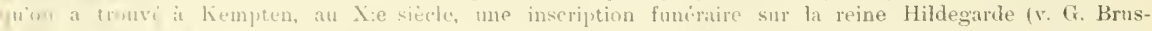

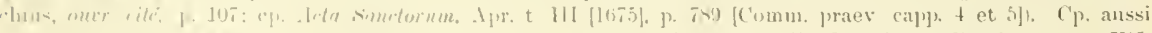

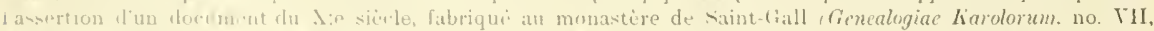

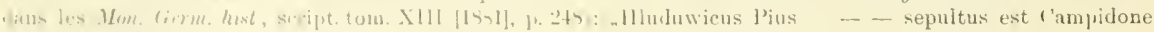
"umi matre" sma".

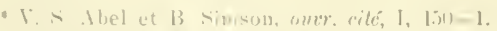

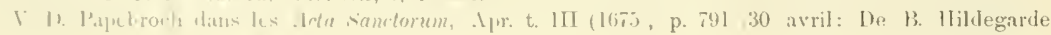

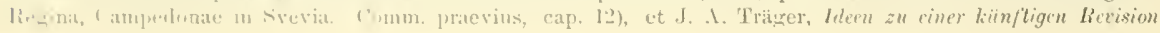


degarle n'a pas fondé le courent de Kempten, assertion fondée essentiellement sur me charte apocrypue, datée le 3 avil $77: 3$, par laquelle entre antres, Charlemagne confirma ume donation de sa femme Hildegarde an convent de Kempten ${ }^{1}$. Si ì cela on ajonte que Taland semble être un persommage entièrement fictif ${ }^{2}$, que les personnages figmant sous les noms de Freydenberg et de Rosinu non Bodmunn ont sûrement été introduits dans l'historre d'Hildegirde pour rehansser l'éclat de denx familles sonabes", que Charlemagne na pas fait d'expédition guerrière contre les Afusulmans de la Terre Sainte ${ }^{4}$, et entin qu'il n'était pas enore emperem en 765 , il est évident que le récit de Birck n'est fondé sur aucun fait historiqne ${ }^{5}$.

über das Alter harls de's Grossen und seiner ersten wehtmëssigcn Formählung mit Hildegard (1s20(1), pp. 30-2. Cp. aussi J. Hibner, Crenealogische Tabellen, t. I (1737), tabl, 25, et L. v. Ranke, Weltgeschichte, t. VI, part. I (1885), p. 181 (il y a ici probablement un simple lopsus calami: "Hildegarde" pour "Himiltrude"; cp. t. V, part. If [185t], p. 113).

1 V., pour cette charte (publiée plusieurs fois, la premiere fois probablement vers le milieu du XVI:e

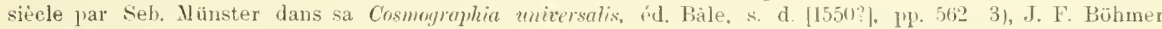
et E. Mühlbacher, oucr. eité, I, pp. 65-6 (no. 157). Peut-être les chroniques mêmes de Birck ont-elles prodnit linscription suivante, tronvée sur une colonne du courent de Kempten et datant de 1trt:

"Hildegardis ll('LXXIIl fundavit

Carolus 1 ('CLXXIY confirmavit

Adrianus bCULXXIY dedicavit

Andelgarins DrLXXVlll inchoavit"

(v. G. Bruschius, ouvr. cité, p. 89; ep. A. Mai, seriptom velerum nova collectio, t. Y [1831], p. 193). -- II est cependant possible qu'Hildegarde ait lait restaurer le courent de Kempten et l'ait richement qloté. Du moins, d'apres une charte (peut-être apocryphe) du l:er septembre s39, Hildegarde a-t-elle fait dejposer it Kempten les corjs des saints martyrs Gordien et Epimachus ir, pour cette charte, J. F. Bühmer et E. Niihlbacher, ouvr. cite; I, 368 [no. 967]; cp. S. Abel et B. Simson, ouvs. rité, I, 451). - V., sur l'histoire véridique du convent de Kejupten, F. L. Baumann, Gieschichte des Allgäus, I (1s,1), 108-10.

" Brusch, dans sa Chronologia Honasteriorum (éd. 16-2), 1p. 91 et 93, dont nous parlerons encore, donne à Taland pour mère Leutburga, seconde femme de Pépin le Bref. J. Hübuer, Genealogische Tabellen, t. I (1737), tabl. 25, dit, daprés notre légenle naturellement: „Talandas - — - soll sich in die Kayserin Hildegard verliebt haben".

${ }^{3}$ V., sur la famille Freydenberg, J. Chr. von Hellbach, Adels-Lexihon. (1825-1826). I, 381 a; stammbuch des blichenden und abgestorbenen Adels in I'eutsrhland, I (1s60)\%. 3s1 n; sur la famille Bodmann, Hellbach,

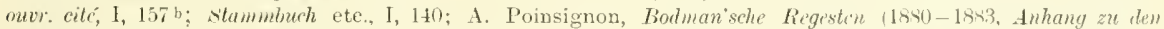
Srhriften des Fereins firr Geschichte des Bodensee's und seiner (Jingebung, fasc. I-XI). Le nom de hosina se rencontre souvent dans cette dernière famille (v. D. Hartard von und zu Hattstein, Ilie IIoheit des leutschen Reichs-Adels, 111 [1740], 75 , 79 et 83), et notre Rosina, compagne I'Hildegarde, a meme trouve une place dans la Germenia topo-rhono-stemmato-graphica saera rt profana ile l'historiographe Gabrie] Bucelinus (t. 11 [1tif?2], App., p. 363: "Rosina Bodman. S. Hildegardis Aulica \& peculiariter dilect. 1. 770"), uaturellement d'après notre légende. - Nous ne saurions dire si flelinde, épouse l'Otto, comte de líescelberg, a vaiment été la suenr dHildegarle. comme le prétenclent Birck et ses imitateurs; v, concernant rette Adelinde. Actr sianrorum, Ang. tom. YI (iel lofin), pp $492+$ (2s aout).

+ Qnil s'agisse d'une expédition en Terre Sainte, c'est ce qui ressort clairement d'un passage de la version de Birck oi ('harlemagne dit: .Nun byu dorh ieh by dem hailigen grib gewesen "(ed. Reiser, p. 44).

s Mentionnons en passant que la méme Hilkegarile a été faite l'hẻrome d'ure légende piéue lans laruelle il est raconté que l'innocence d'Hildegarle, yni avait été accusée d’adultère, fut démontrie par le fait yuun rayon de soleil supporta ses gants, qu'elle avait otés pour faire sa prière (v. IList. s. Irmulfi Mettensis, p. 36 [Mon. Germ. hist, script. tom. XXIT (1si9), pp. 53t-5]). Le meme miracle est, l'ailleurs, raconté à propos de la piense Cumégonde, épouse de l'mupereur Henri II (morte en 103:3): r: Titu sanché Cunegundis, cap. 8 (Mon. Germ. hist., script. t. IV [18+1], p. 823). 
lat lénemle que Birck avait fabrigute en l'honneur d'Hildegarde ne se serait sans houte pas répandue hors des cercles intéressés en Sonabe ${ }^{1}$, si un historiographe

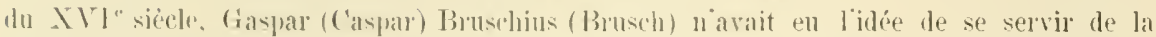
chronique de Burck, pour l'histoire de Kempten. lans som onvage intitulé Monasteri-

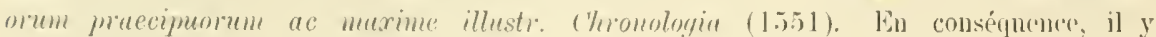

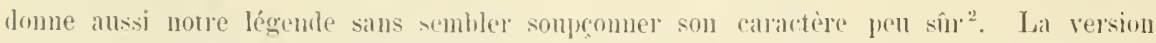
abrégée de Brusch diflère un pen de celle de Birck. lantem ayant voulu compléter et corriger quelques détails de sa source:

Fipisodes: (a) ('harlemagne part pour une guerre contre les Sixons; Hildegarde, rondamnée it ètre noyie, ist it temps prevenue du danger ${ }^{3}$; (e) elle se rend it lome, sans $y$ i.tre appelée par le pape; $(g)$ Taland se confesse fileinement tout de suite.

Une tradnetion allemande de la version latine de Brusch nous est connue par

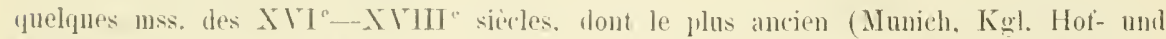
Staatsbibl., corl. germ. 5\$23) porte le titre: Beschreib vml stuiffung desz Fürstlich Gottshrusies zu kempten *.

Tres la fin du XYl" siecle. un antre historiographe ablemamu, Martin Crusins (1506-1607), laconta, dans ses Annales suevici (1595) ${ }^{5}$ : la légende d'Hlilegarle d'ilpres la rersion de IBrusch, tout en se servant en même temps de la chronique de Birck ${ }^{6}$. Toici deux traits par lespuels la version de Crnsins dilfère de celle de Brnsch:

Fipisodes: al Hildegarde est jetie dans le Heuve ${ }^{\overline{7}}$; (g) Taland ne confesse pas tout dabord son crime envers sa belle-soeur?

On sait pu'Hillegarde est regardée comme la patrome de liempten: la rille est encore pleine de ses souvenirs (entre autres, sa statue lécore une fontaine sur le "liesidenzplatz", et non loin de Kempten

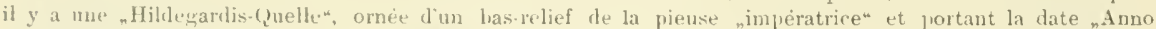

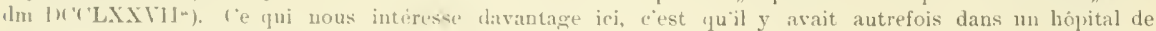

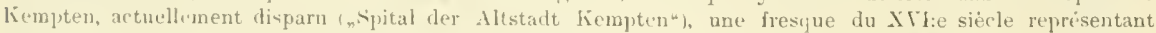

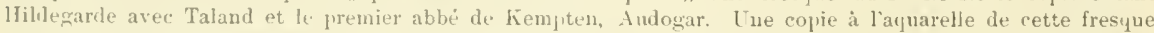
est entre les mains de .J. Nartin Leichtle, a Kempten. ('p. F. L Banmann, Geschichle des Allgäus, I (1os1), 75, oul se trouve une reproduction de cette ropie.

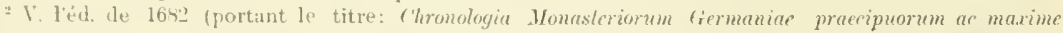
illustrium), 1'p. 93- 7 . Brusch dit aroir tronvi notre ligende ('ampidoni in reteribus monasterii annalibus". ('lam admonita". Bruseh a jent-être mal interprété sa source, qui dit (éd. Reiser. p. HHı: ${ }_{n}$ lo verhyes\% sy [Hyltgart] vnser frowen, las sy jr vschulff [se, dem wasser|, so wölt sy durch jren willen ain gotzhusz buwen. Also half ir unser frow isz barnach kam sy an ainen hoff _ - -

Notre histoire s'y lit fol. $1 r^{\circ}-1 j r^{\circ}$ (sins pagination): litistoire des abbés de lienpten y va

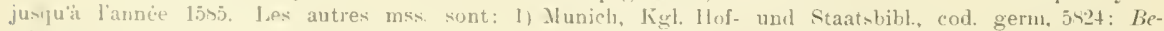

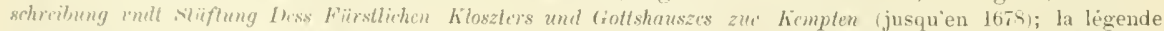

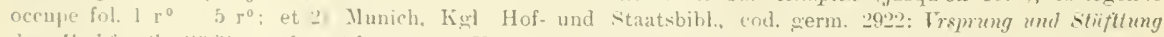

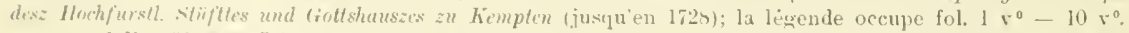
$\mathrm{X}(165: 4,15$

3 l' 3 . 316 h Lib. XIJ, cap. 11. Le passage a éti rimprimé par J. Ph. a Vorburg, Historiae,

- ('rusius hai-meme (1). 3Iti) dit avoir tiré la légende dillildegarde de Bruscì et d'un n. Ser. vetere libro Germanico".

" "p. la version de J3irck. I'rusius ajoute lui-méme entre parentheses: ${ }_{n} \mathrm{rt}$ est in M. Sc. libro".

- Ci. la vursion de Birek. 
La légende d'Hildegarde se rencontre aussi dans la trutuction allemande des Amales snevici par Joham Jacob Moser: Schcäbische Chroniti (1733).

Une fois entrée pour tont de bon clans la littératnre historigne de l'Allemagne, la légente d'Hildegarde $\mathrm{y}$ fait sou chemin pentant les siècles smivants, tontefois le plus sonvent à titre de tradition loutense on fabulense. Ainsi, nous retronvons notre légrende, sans doute d'après Brusch, quoique lantem ne eite que les ,Amales Campridonenses", dans la Gülichische Chronie (1611) d'Adelerius Erichns². De Crusins, d'autre part, vient la version quelque pen abrégée de Clurstoph Lehmann dans sa Chromicu der fieyen Reichs Stadt Speier (1612), laquelle porte le titre: Von Talandi Königs Caroli unchelichen Bruders grosser Untren. so er dem Lönig und seinem Gemahl Hildegurt eruiesen". Puis, nous rencontrons notre légende dans un onvrage inédit de M. Johann Megglin. Aüff vond Vidergang dess Fïrstlichen Sliiffts vmu Gottshairsa Kempten $(1632)^{4}$. C'itons ensuite les onvrages historiques snivants qui, plus ou moins longuement, domnent la légende d'après Brusch: Historia Curoli Magni de Johamnes Joachim Frantz $(1644)^{5}$, les Acta Sunetorm $(1675)^{6}$, les Amules Ecclesiastici Fruncorm de Charles Le Cointe $(1676)^{\top}$, La Monurchie Sumbe, listorique, chronologique it genéalogique de France de Modeste ie s. Amable $(167 \%)^{\mathrm{s}}$, les Amales Imperii Occidentis Brunscicenses de Gottfied Wilhelm Leibniz (mort en 1716) ${ }^{9}$; etc. etc. Même mu historien du XIX" siècle, Joh. Bapt. Haggenniuller, raconte encore notre légende dans son histoire de Kempten (Geschichte der Stalt um der gefüsteten Grafschuft Kempten, t. I, 1S40) ${ }^{10}$.

Naturellement notre légende ne se rencontre gras senlement dans des ouvages l'histoire, où les anteurs tâcbent plns ou moins sérieusement de ne raconter que des faits anthentiques; nous la retronvons anssi dans des onvages d'un caractere moins sérieux, des recueils d'anealotes et d'histoires éditiantes. Les sources sont tonjours

1 Pp. $269-70$.

${ }^{2}$ Fol. $191 \mathrm{r}^{0}-192 \mathrm{r}^{0}$ (livre IV, chap. I:er).

${ }^{3}$ V. la t:e id. (1711), pp. $213-4$ (livre 11I, chap. XXXI).

+ Ms. Munich, Kyl. Hof- und Staatsbibl, eod. germ. 5826, fol. $7 v^{0}-10 v^{0}$ (chap. 4 -5). Lautenr, cure d'Undersriedt (Interried, Bavière), cite Brusch et (rusius. Une copie de la chronipue de Mlegroliu fut faite au commencement de XIX:e siécle (ms. Nunich, Hof- und Staatsbibl., corl. germ. 5124); notre linende y occupe fol. $6 \mathrm{v}^{\circ}-9 \mathrm{r}^{\circ}$.

${ }_{5}^{5} \mathrm{P}_{1}$. 44-5. Frantz renvoie it ,antiquorum monasteriorum anuales".

- Г. Аpr. t. ПI, p. 792-3; él. de 1,66, p. $80^{2}$ (XXX aprilis: De B. Hildegarde Regina, Campedenae in. suevia. Comm. praevius, cap. 20). Liauteur de la brève analyse, qui était laniel Papebroch, regarde la légende comme fabuleuse.

${ }^{7}$ T. VI, pp. 232-3. Le Cointe (Cointius) regarde expressément la légende comme labuleuse.

\& T. II, 1p. 4 2 -3. L'auteur, qui cite Brusch es Crusius, regarde la légende comme fabuleuse.

${ }_{9}$ V. l'éd. de Pertz $(1843-1846)$, t. I, p. 108 (Ann. 783, cap. 2). Ce n'est qu'un bref résumé.

$10 \mathrm{Pp}$. 20-1. Haggenmüller cite des Chroniques, mais il semble, par certains ditails, que ce soit louvrage de Brusch qu'il ait utilisé. 
Brusch et Crusius on $u n$ de lemrs snceessents. Déjic en $156 ; 3$, nons trourons notre légrende soms le titre Von liöriy c'urolo magno pine uare histori dans le célèbre ou-

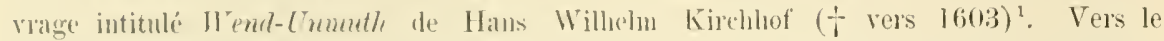

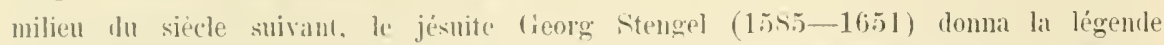
d'apres Brusch dans son ourrage intitulé Opus de Iudicis Dirinis quae Deus in hoc mundo exercet ${ }^{2}$, dont il existe anssi ume trablution en allemand de l'amnie $1712^{3}$. Puis, nons retronvons notre légente dans nu onvage de Fortunatus Hüber: I'nsterbliche Gedächtnes De' iontrefflichen Geschichten, Heitigen Stifftungen vnd wunderlichen stands Tevänderungen, I'elche dem Buyrischen hohen Alter haben einyedruckt

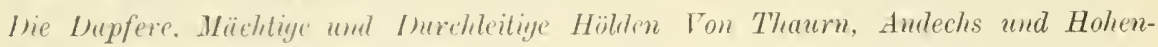
unth - - $(1670)^{\prime}$. ef la version de Hüher a été ntilisée. en 1655, par Anton

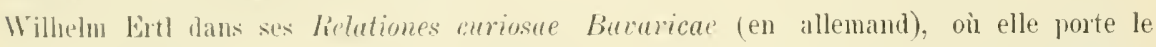
titre: Simprangemle traschull Hildegurlis ${ }^{5}$. Whtl a introduit quelques changements dins sil version, complure arec celle de Bronsch, dont voici les princiuaux:

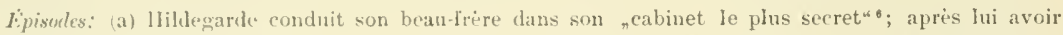
parli amicalement, elle sost comme pour voir si queline domestique les a suivis et se hatte de fermer la porte de lit chambre; Taland est lajssé quelques jours sans nourriture; (harlemagne ordonve non senlement d'arracher les yeux it son ćpouse, mais aussi de la précipiter ensuite dans une fosse ("Pfütze ${ }^{\mu}{ }^{7}$; (e) lamie sidpelle "Rosina von Pottmann* (jrononeiation sul-allemande).

(¿uclunes années aprés Erttl, le romancier Eberhard Werner Happel (1647-1690) publia, d’après Frichuns, la première partie de notre légende, jusqu'à l'emprisonnement de 'Taland, dans ses Grösseste Denkü̈rligkeilen der N'elt oder so genandte Relationes curiosae sous le titue Die listige Keuscheit". Puis nous trourons notre légende dans nu des nombrenx ourages dn célèbre écrivain populaire Martimus ron Cochem $(\dagger$ en 1712): Auserlesenes Gar unmuthiges, und seh. mutiches History-Buch. Cette version.

V. Kid. dösterley (1569), t. II, pp. 47-52 (livre 11, no. 23). Kirchhof renvoie aux Annales de Limplin, mais sa version parait bien itre basie sur celle de liruseh. Le nom de la compagne d'Hildegarde a éte corrompue en . Fosina ron Bodinen".

2 V. l'ouvrage posthume de Stengel: Mundus Thenreticus divinorum Iudiciorum in quatunr partes diygstus - - (Cologne 16\$2). purs tertia, cap. XLIX, no. IX (pp. 187-9). Lihistoire dHildegarde se tromvait d'ailleurs peutetre líja dans ymelpue ouvrage antirieur de Stengel, car on sait que lopus de ludiriis llivinik n'ust yu un recueil de plusieurs de ses ourrages parus antérieurement.

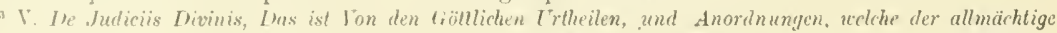
Foll in dieser Tett vebet - - , partie 111, pp. 517--21 (cap. 49, no. 9: W'ic die . Inklag der Kayserin Hildegardis auff din Lrluber yifullen).

1'p. 184-94 (Buch If, Abth. 11). Hiiber indique Brusch coume sa source.

sp. 103-6 (Rel. cur. LAH1). Ertl ne mentionne pas Hiber, mais certains détails du récit ne l:tissent pas dle doute sur sa source

- Lhija dans Hijher il n'est plus question de la construction d'un local spécial.

7 Dija Hister donne ce dernier trait.

- T. V. I:ere jartse (1tige)). p1. 161 - Le titre lie listige lieuschcit est commun à notre histoire et ¿ une autre, qui la précède, concernant Ludomilla et Ludewig, dnc de Bavière. 
qui porte le titre: I'on der unschuldigen Behlagung, langwibrdigen Elend, und ondlicher Evhöhung der seligen Kayserin. Hildegardis', présente certaines altérations de la version de Brusch:

Eipisorles: (a) le rendez-vous est pris dans une tour, située sur les murs de l'enceinte du palais; ('harlemagne revenant frajple Hildegarle et donne l'ordre de la decapiter; les soldats, inus de pitié, prennent ses habits et la laissent en vie; (e) „Rosina von Bodnn " est la camérière dHildegarde et vient la chercher lans la forêt; les deux femmes ont appris dans leur jeunesse à fabriquer, avec des plantes, tontes sortes de remédes; (g) Charlemagne envoie d’abord chez Hildegarde un domestique pour la jrier de venir guérir Taland, mais elle refuse; Taland ne eonlesse pas tout d'abord son crime envers Hildegarde, de sorte que le remède ne produit aucun effet; il rloit done se confesser une seconde fois; (g) en quittant Rome, Hildegarde reçoit du pape antant de reliques qu'elle pent prendre aves un doigt, et lieu lui donne pour cette oreasion une force surnaturelle ${ }^{3}$.

Nous retrouvons encore notre légende dans me des nombrenses anures dn célèbre prédicateur Abraham à Sancta Clara (- Hans Llrich Negerlin, 164t-1709): Abruhamische Lauber-Hätt, publiée après sa mort, penlant les années 1721-17235. La version d'Abraham à $S$. Clatra est très courte, mais présente quelques imnovatious (comparée avec celle de Brusch):

Personnagcs: (3) Talardus ${ }^{6}$. Épisodes: (a) il n'est pas question le la construction de l'appartement seeret („das innerste Cabinet“); llildegarde poste des gardes devant la porte; (e) l'amie d'Hildegarde n’est pas du tont mentionnée; (f) le beau-frère devient seulement aveugle; (g) aprés la réconriliation des époux, le beau-frè devient de nouveau avengle; il n'est pas question de bannissement.

Enfin, en 1720, le jsentonyme Jasander donna la légende d'Hildegarde dans son onvage intitulé Der in fremden Lämlern sich lany aufychaltene, numehro aber wieder angelungte Teutsche Historien-Schrober, velcher allerhand Curiositäten, lustige. und lesenswïrdige Beyphenhriten, für ulle diejemigen, so Liebhuber der Novitäten sezm,

1 V. l'éd, de 1732, pp. 931-49 (Ter sechste Titel. Fon vilen trübscligen Persohnen. - - Die Drey und sibenzigste History). Dans la première édition, de 1687 1692, cette histoire ne se tronve pas, mais à sa place on $y$ voit figurer une version du mirarle de la Vierge daprès Vincent de Beauvais (v. ci-dessus p. +1 ). Pour sa version d'Hillegarle. Afartinus von Cochem cite comme autorité Stengel, mais il y a des passages qui montrent clanement 'ju'il a aussi utilisé Crusius ou un les auteurs qui ont snivi celui-ci. - En 18H, il a étú imprimé (à Passau) une idition a part de eette version de notre légende sons le titre: Ifildegardis, die

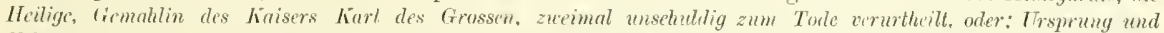

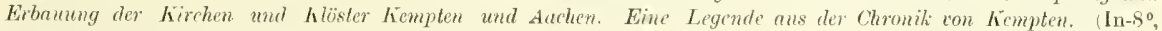

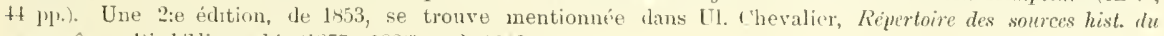
moymen-âgr. birr-bibliographie (18Ti-1856), col. 1063.

? P. la version de Crusius (ei-dessus p. 70).

3 rette anecdote est aussi tirée de Crusius, ouvr. rité. J. 319.

* T. II, pp. 100-2 ('hap. VIII: Die gleiche Hiedirergeltung, no. 4).

${ }^{5}$ La licence d'impression avait été donnée dejit en 1717 ; v. Th. G. v. Karajan, Abraham a sancta Clara (186i), pp. 360-1.

${ }^{6} 11$ est eurieux de noter que J. Fr. Pleffuger, qui, dans son onvrage Cormus juris publici, de l'année 17.54 (t. I, p. I'stb; lib. 1, tit. XV, \$ 16, e), mentionno notre légende en citant Isruseh et Lehmann, ernploie préeisément la forme T'alardus. 
mit sirh getracht'. ('ette rersion présente les divergences suivantes avec la version de Brinscli:

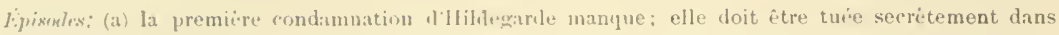

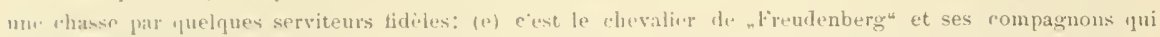
fout partir four liome llildegarde avee sir camiriere, thist le nom n'est pas dit: (f) Taland arrise a Rome,

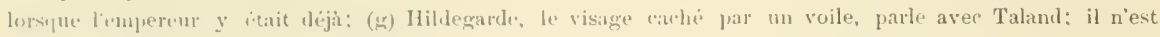
fas question de la fondation du rousent.

1 côté de tontes des versions historiques ou anecdotiques, il nous faut mentionner me murr dramatique en vers latins, basée sur le récit de Brusch. C"est la

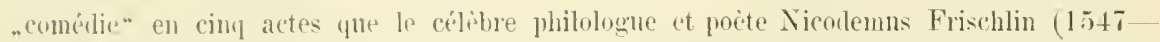

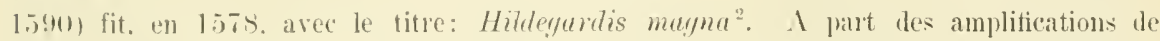
létail. rette . comédie” rent tidèlement la rersion de Brusch. Elle fut rejrésentée i Stutterart to 1:er janvier 1579 a l'occasion de l'avènement an trône de Lonis, du de Wurttemberg:", et l'on sait quelle a anssi été jonée lepuis".

I.e frère de Nicodemus F'jischlin, Jacob Frisehlin. tradnisit en allemand la pièce de son frire sous le titre: Hildegardis Magna. Eine schine Comedien von Frau Hildegurdin Hertzoy Hildebrands in Schuben Tochter, Keysers Caroli Magni Gemahlin .

'Histoire 3!. Liexemplaire du Musce Britannique, dont nous nous sommes servi, nest pas date; le catalogue tle la bibliothéque donne l'année 1738 avec un point dinterrogation. La légende s'y trouve pp.

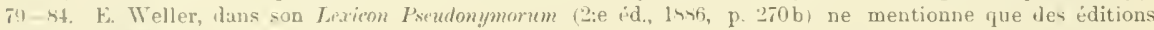
de $1720,1729,1729,1730$ et 1750 .

${ }^{2}$ Xous avons consulté une édition de 1585) (now paginée [Ntrasbourg], in-12). Tans l'édition de l604 de l'Operorum poeticorum pars scenica de l'rischlin, notre ncomedie“ se lit pp. I49_-206. Pour les éditions des

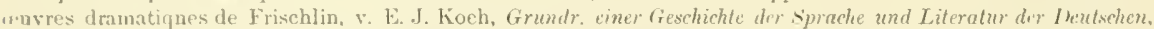

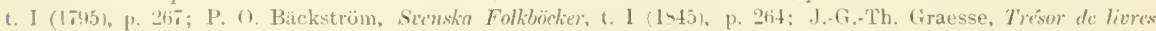

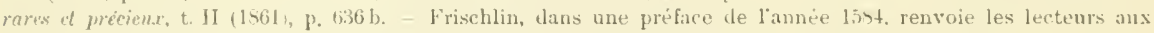
amnales rampridonensers, mais il est bien improbable qu'il soit allé au-delà de la version de Bruseh.

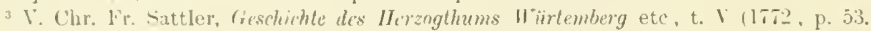

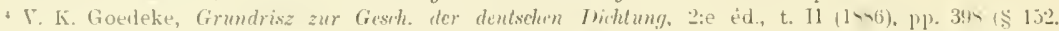

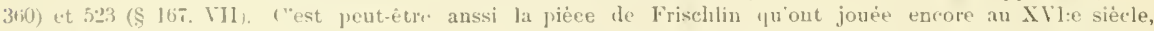
une fois par an, les ecolicrs de la ville de Kempten (v, J. li. Haggenmüller, Fesch. der stalt und der gifür-

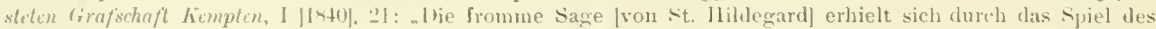

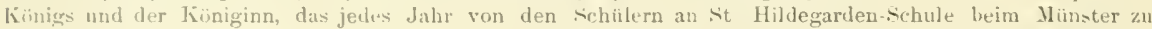
Kempten an F'assnarht aufgeführt, uml bis ins spchszehute Jahrhundert beibehalten wurde", et J. von Hor-

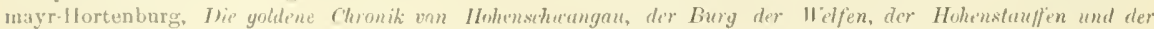

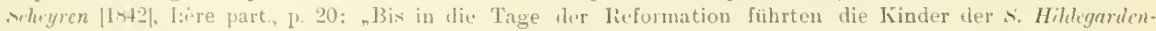
schule beim Ilïnster zu liempten um Fassnacht das sjpiel vou der frommen Königin auf*).

s l. pour rette trafuction, imprime en 1599 i strashomg, et que nous n'avons pas vue, J. I'les-

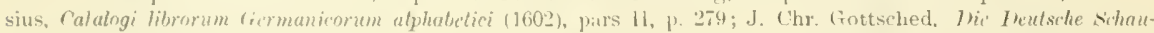

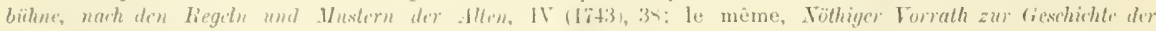

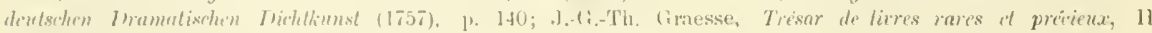
(1>61), 637 a. 
Il nous reste à mentionner les pérégrinations de notre légende comme conte populaire. Sons cette forme elle présente deux versions principales, dont l'une est apparentée de très près à la version de Brusch. Il est naturellement impossible de dire par quelle voie l'histoire l'Hildegarde est levenue me légende courante dans la bouche du penple. On pent tonjonrs se fignrer comme sonce première quelque sermon le prédicatenr, où la légende, empruntée di l'un des onvrages historiques on anecilutiques dont nous avons parlé ci-dessns, aurait été intercalée comme „exemple“ instructif. En ancun cas, ces versions popnlaires de la légende d'Hildegarde ne penvent provenir directement de la chroniqne de Birck on les traditions locales créées par elle, car les contes populaires d'Hildegarde présentent quelques traits qui ne se trouvent pas dans la version de Birck, mais bien dans Brnsch. Fn ontre, ces contes sont, du moins en partie, des contes rhénans, et c’est Ingelheim (près de Mayence) qui y est désigné comme l'endroit où Charlemagne aurait laissé sa femme en partant pour la guerre contre les Saxons.

Celle des denx versions du conte populaire qui est le moins éloignée de la version de Brusch a dû exister an moins vers le milien dn XVII ${ }^{e}$ siècle, car, dans ses Svenska Folkböcker, I’. O. Bäckström a signale une édition suédoise de cette version datant de 16s9 et portant le titre: En lustigh och sanfärdigh Historia om Drottning Hildegardis, Caroli Magni Hufru (sic), och hennes Styffbroder Talando". Depnis, il a part en Snède bon nombre d'éditions de ce conte populaire: il y en at de $1699^{2}$, de la seconde moitié du XVIII ${ }^{\mathrm{e}}$ siècle $^{3}$, de $1809^{4}$, de $1810^{5}$, de 1855 , de $1858^{6}$, et probablement encore d'autres que nons ne connaissons pas. Toici les principaux traits par lesequels la version sncidoise de 1689 diffère de la version de Brusch:

1 V. Bäckström, ouvr. cité, t. I (1545), pp. 264 et 266; cette version est reproduite, sons une forme modernisée et avec le titre Hildegardis och Talandus, ibid. 1'p. 266-5. Dans l'édition de 16ss?, le conte d'Hildegarde est précédé dı conte des (vulre marchands (Fyra Fiömän; le thème en est apyarenté à celui du Roman de la Violette). Sur le fenillet de titre commun des denx contes, celui d'Hildegarde est annoncé en ces termes: Ther hoos och en annan sanfürdig Historia om Irothing Hithegardis, Caroli Magni Husfru, och lennes styfbroder Talando. - Par une curieuse mejprise (confusion de sein et illr?), Taland est, snr le titre des éditions suédoises, nommé „demi-frère" d'Hildegarde hennes pour hans).

2 V. Bäckström, nutr. citć, II (1848), ofversigt af svenskit Folk-Litteraturon, pp. 6-7. Le titre de notre histoire $\mathrm{y}$ est exactement le même que dans l'édition de 16s9. mais aprés I'histoire d'Hildegarde' $\mathrm{y}$ viennent encore celles d'Hureule et d'Ulfspegel.

${ }^{3}$ V. Bäckström, ouvr. cité, t. I, p. 266 (le feuillet de titre manquait dans l'exemplaire que Bäck. ström avait sous les yeux). Cette edition du conte d'Hildegarde, „uli parte, daprés Bickström, le titre: En

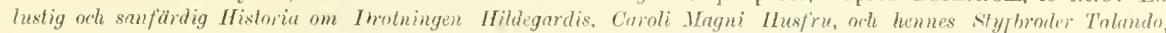
est précédée dn conte des Quntre Harchunds et suivie de cenx diflercule, dillispegel ot de Néron.

+ V. Bäckström, ouvr. cite. 11, i)fversigt etc., p. 7, et $11 \mathrm{j}$. Linnström. Svenskt Bolilexikon. Iren 1s,30-1s(is (1883-1894), 1, 642 (no. I69; édition partille is celle de 1699).

s V. Linnström, oucr. cité, 1, 642 (no. 169, édition pareille à celles de 1699 et de lxn9).

- V. Linnström, nurr. cité, 1, 652 (no 293). Nans ces leux dernières editions l'histoire d'Hildequrde (Trottning Itildegarlis) est pricédée de celle de la Princesse Blanch-Xeige. 
I'rsonnagres: (t) un chesalier, venant de lit part de la comtesse Idelgunda, serur l'Hillegarde.

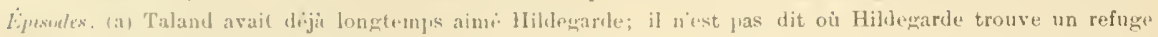

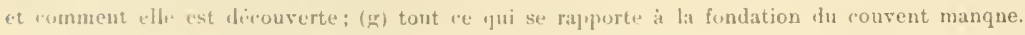

En Allemagne, le conte populaire d"Hillegarde resta incomu pour le grand public jusqu'an XIX" sircle, du moins, l'après re que nous avons pu constater. Ce ne fut quin 1S1; que lhistorien Aloys Wrihlelm Sichreiher (1763-1841) en publia une version, dans son Hamlluch fïr Risisende am Rihein von Schafhausen bis Holland, sous le titre: Hillegam ${ }^{1}$. Ia meme version parut aussi dans un extrait de cet ourrage par le même autemr: Ausuahl der interessantesten Sayen aus den Gegenden des Rheins

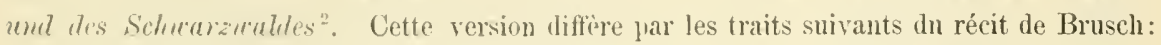

I'ersonnages: (t) le nom du chevalier n'est pas dit. Fipisodes: (a) Charlemagne séjourne à Ingelheim; il néct fas question dus trois portes de la maison du rendez-vous; le fleuve ou Hildegarde doit être jetée est le Rluin; (f) la malarlie le Taland niest pas dite; (g) tout ce qui se rapporto à la fondation du couvent le Kémpten manque.

liamme snivante (1817), l'historien Niklas Togt (1756-1836) publia me version Im peu différentr de re conte dans ses Rheinische Geschichten und Sagen ${ }^{3}$. Voici lar quels traits cette version diffère de relle de Brusch:

l'rronnages: (t) un des courtisans te ('harlemagne. Fipisodes: (a) la maison dn rendez-rous nest fras construite expris: il n'est pas question des trnis portes; la premiere condumnation manque; (e) lo personnage de Rosina von holmann manque; (f) Taland ne devient qu'avengle; ce n'est qu'à Rone qüil apprend l'existence de la femme qui guirit tous les manx; (g) Taland est pardonné; les détails sur la fondation du couvent de kempten manupents.

1 Pp. 475 - S (no. XI). Dans la 3:e ébition (s. d.), le conte se lit pp. 514-6. La seconde édition, iggalcment saus date (d'apris kiayser, Vollstïnd. Bürher-Lcxiron, t. V [1835], p. 15t a, elle est de 1818), qui porte aussi le titre: Inleilung auf die nützlichste und genussvollsle Art dem lhhein - - zu berciscn. donne notre ronte lans une partie avec pagination ì part, intitulée Volkssagen aus den Gegenden am Rhein und am Toumus (pp. 4i-51)), 'fui manqu dans certains exemplaires de cette edition du Handburh. Nous avons en.

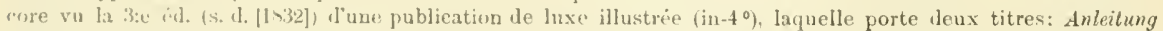
ete. leomme ha seconde eddition du Handburh) et Tollstïndiges Gremälde der Rheinlande von Schathausen bis Iinlhnu, und der whönsten amliegenden Gigenden; notre conte se lit ]p. 185-6. - Une traduction française du

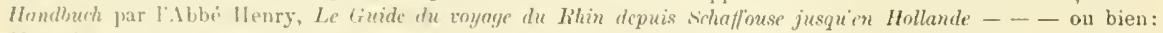

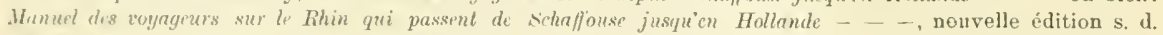

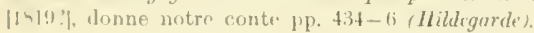

${ }^{2}$ V. la $2: u$ inl. (1-29), portant le titre: Sagen aus den Gegenden des Rheins und des Schwarzwaldcs, pp. 53 (j (no. 11: Mildegard): 3:e id. (1445), l:ire partie, pp. 41-3 (eilition portant le titre: sagen aus den Rluinyrginlen, dim sihuarzalde und den logesen). Ine traluction française de cette version d'Hildegarde se lit

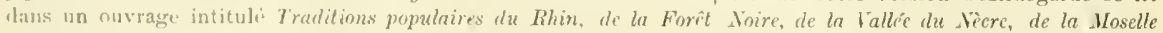
et du Taunus. Prublices par ll:r le Conseiller aulique sihreiber el aulres (Heidelberg, s. d. [1825?]), pp. 29-31] (avec me estampe reprisentant la scène do la reconnaissanse à l'uglise Saint-Pierre). P., sur l'auteur et

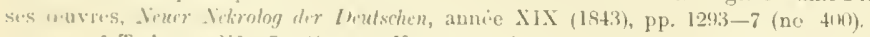

${ }^{3}$ T. 1. 11). 215-7. Comme Vogt renvoie anx , Jahrbiicher des Klosters zu kempten", il est probable 'jüil a conmu la version de lirusch.

\$ Comme, dans lit version du conte rhinan que donno Selureiber, toute mention du couvent de

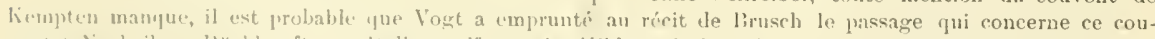

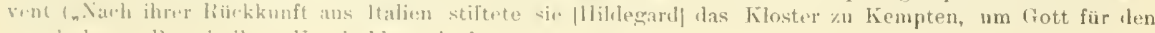

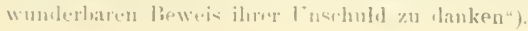


Enfin, eı 1818, parnt en Allemagne une troisième version populaire de ce même conte d'Hildegarde: dans les Deutsche Sayen des Frères Grimm ${ }^{1}$. Cette version diffère par les traits suivants de la version de Brusch:

Personnages: (4) le chevalier de Freudenberg, au service de la comtesse Adelgund, sorir d'Hildegarcle. F́pisodes: (a) Hildegarde, conlannée à être noyée, trouve un refuge chez une de ses anies; (e) la compagne est une "Edelfrau“, Rosina von Bodmer; (g) rien concernant la fondation du couvent.

Par la forme du nom donné à la soem d'Hildegarde, on voit elairement que c'est la version populaire reproduite par les frères Grimm qui avait tronvé, au XVII ${ }^{e}$ siècle, son chemin jusqu'en Suède.

En comparant entre elles ces quatre versions du conte populaire d'Hildegarde, on voit qu'elles ont dî avoir comme source commune une version populaire qui ne différait que par quelques omissions de la rersion donnée par Brusch.

Grîce aux versions populaires données par Schreiber, Voğt et les frères Grimm, le conte d'Hillegarde semble avoir açnis une certaine notoriété dans les cercles néoromantiques de I'Allemagne de la première moitié du XIX e siècle, de sorte qu'il a même fait naître des ballades plus on moins pathétiques. C'est ainsi que Karl Geib (1777-1852) a emprunté à Schreiber l'idée de la ballade Hildegard dans son onvage Die Tolkssagen des Rheinlandes (t. I, 1828)². (ieib y antroduit quelques changements:

Personnages: (t) un chevalier Egbert, au service d'Adeline, scenr l'Hildegarde. Épismles: (a) le château où les rendez-vous devaient avoir lieu est déjà prêt; (e) à Rome, Hilılegarde se fait appeler Emma.

Plus tard (en 1836), toujours d'après Schreiber, Gieib raconta le même conte en prose, arec beancoup d'amplifications de détail, dans sun onvrage Die Sugen und Geschiehten des Rheinlundes, sous le titre Die Königin Hildegarl ${ }^{3}$. Mentionnons parmi les additions la senle qui ait quelque importance:

1 T. П, pp. 102-4 (no. 437: Hildegard). Les anteurs renvoient aux "Annules cumpiloncnses", probablement d'aprés N. Frischlin, dont il citent la version. - I tans la traduction française de ces contes par N. Theil (Traditions allemandes recucillies et publiés pur les Frères Grimm, 1838), Hildegard se lit t. 1I, pp. $120-3$.

${ }^{2}$ Pp. 51-9. Il y a 43 strophes, dont voici la premiere:

"Kaiser Karl des Grossen Schaaren

Kommen froh in's heim'sche Land

Aus Hispania, ruhmgekrönet;

Doch die Kriegsdrommete tönet

Neu, sie rufend an der Weser Strand*.

(Avec une gravure représentant la scẻne de la reconnaissance à l'église). - Sclureiber, dans la preface de ses Sagen aus den Gegenden des Fiheins (2:e éd, p. V111), attestait lui-même l'origine des poésies de Geib: . Was von diesen Sagen friaher [c'est-à-dire dans le Handbuch] gedruckt war, hat Herrn Carl reib Stofł zu Balladen und Romanzen geliefert".

${ }^{3}$ Pp. 297-306; nouv. éd. (1850), pp. 417-29. Geib y renvoio aux annales de Kempten, daprès Vogt pent-être. 
Fivisulo (g): llildrerarde fait batir le convent de Ktmpten en reconnaissance de ce rue son innocence at iti prouvies.

Lous fourons signaler encore deux autres ballades allemandes traitant de la romlammation injuste d'Hilelegarde.

Liune de res ballades, intitulée Legende, a pour antem Friedrich Lam (psen-

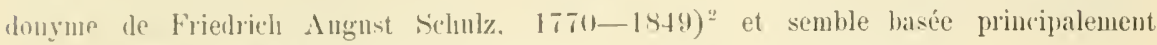
sur la version de $\mathrm{Tout}^{3}$. Nous annotons ici les primeipales divergences:

Pirsonnalys: fl manque. Ejpisods: (a Charlemagne rivide a Worms; ce sont les bourreanx mêmes "fili, tomohís de compassion, laissent llildegarde intacte; (e) llildegarde est aceompagnée à Rome d'une

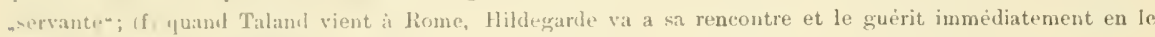
touchaut; 'Talaul se coufusse alors spontaniment; il lui faut aller en exil.

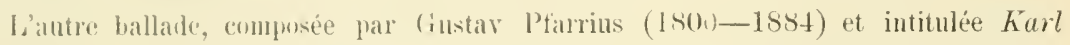
mul Hildeyard, est fundée sur la version de Selneiber. Toici les principales dirergences:

Fipisncle: (a) Taland, secritement anourenx d'Ifildegarde depuis longtemps, aurait voulu la fuir en fartant four la guerre, mais C'larlunagne, yui ne soupçonne rien, ne le permet pas; c'est par peur, menacúe frar 'Taland, ju'Hildegarde a recours à la ruse; sur le point d'être jetée dans le Rhin, Hildegarde est sauvée par deux chevalirrs et conduite au chateau de son frère: de lì, devenue trop connue par ses cures miraculouses, elle se rend stule ì lione (l丷ẹpisole de la seconde condamnation manque done); Taland devient seulement aveugle; ${ }^{6}$ il nest pas question de confession.

('ette addition est probablement empruntíe i la version de Vogt (v. ei-dessus p. 76).

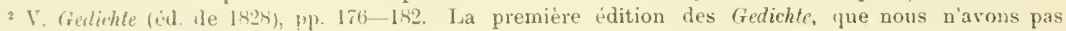
vue, date ile 182t. La version de Laun avait d'ailleurs dejả été publiée dans l'ouvrage de Chr. Kuffner,

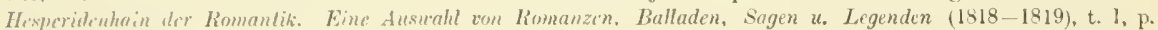

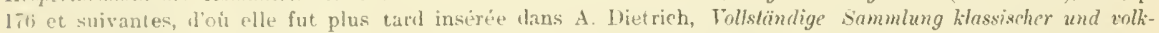
thämlicher deulseher Legenden ans dem 15. und 19. Juhrhundert (15:8; = Braga. Tollständige Sammlung klassischer

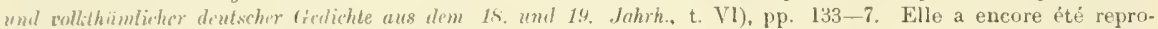
Juite dans le I'octish her Hunsschatz des deutschen Volkes d. (1). L. 13. Wolff (1539), pp. 391-2 (12:e éd. [1S49], 1). 467- 4, ainsi une par A. Schöjpner, sagmhurh der Bayerischen Lande, I (1552), 30-2 (口o. 29: „Hilde.

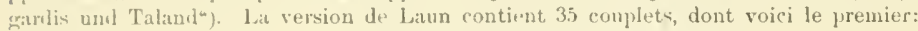

Her grosse Karl, or sass einmal

Zu Worms in seines Thrones Saal,

lind zwischen firafen und Herren stanil

Dicht vor dem Throne Herr "Taland".

3 Lann ronnaissait probablement anssi le récit do Brusch. Ainsi, il eite en note (ésl. 1828, p. 182) la devise do Kimpten: Campialona sola judicat ense, stola, mentionnée dans l'ouvrage do lirusch (éd. 168:2, p. 102). + Trait primitif. altiré dans la rersion de Vogt.

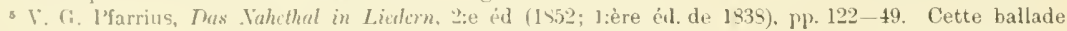
a Ati. ruproluite dans le Pontixcher IIausschatz des deutsehen Volkes de O. L. B. Wolff, 12:e ed. (15t9), pp.

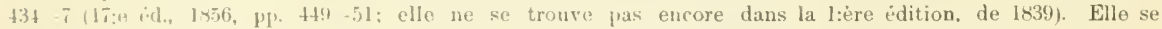
(omprosu de 6.3 conplets, grompes en 6 sections. En roici le premier couplet (nNibclungenstrophe"):

Wie rast ther iturm in Eichenwald, wie sanst der Wirbelwind!

Wie tobet durch die Gaven der Sachse Wittekind!

Die frink'schen Burgen rauchen, die Kirchen sind \%erstört,

1)ie Priestur sind gefallet; solch Wüthen war nimmer erhört“.

l'farrius parait aussi avoir utilisé la ligende dans son poème épique Kurlmann (IRłl), que nous n'avons pas

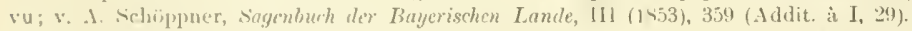

- Comme, dans Schreiber. la maladie n'est pas indiquie, il est pnssible qu'il y ait ici influence de guelịne autre version populaire ot Taland devicnt aveugle (p. "x. la rersion de Vogt). 
A côté des versions du conte populaire d'Hillegarde dont nous venons de parler, qui sont tontes apparentées a'assez près à la version de Brusch, il existe, comme nons l'avons dit plus hant (p. 75), une antre version qui s'éloigne lavantage de la version de Brusch, mais qui semble cependant n'être qu'un remaniement de la version publice par Schreiber. Cette version secondaire, pnbliée en 1837 par A. Reumont dans ses Kheinlands Sagen, Geschichten und Legenden sous le titre: Die Künigin Hildegurdis ${ }^{1}$, diffère par les traits suirants de la rersion de Brusch:

Personnages: 4) manque. Épisodes: (a) Charlemagne réside à Ingelheim ${ }^{2}$; Taland avait longtenps récu à la eonr greeque, ce qui arait eu une mauvaise influenee sur son carartère; rendez-vous est pris dans une chambre secrète du palais; il n'est question que d'une porte; l'épisode de la promière condamnation manque $^{3}$; les bourreaux qui sont chargès de tuer Hildegarde sont mis en fuite par une demoiselle d'honneur de la reine, qui, eachée dans un arbre, les effraie en les menaçant le la colére de l)ien; (e) ('est dans la demeure d'un ermite que les deux femmes vivent quelque temps; à Rome, Hildegarde se fait nommer „Frau Jolorosa"; (f) Taland devient senlement areugle; (g) Hildegarłle parle elle-même avec Taland, qui avoue tout de suite son erime; il se confesse anssi devant Charlemagne, qui, furieux, veut le tner; Hildegarle se fait alors connaître, guérit Taland et obtient son pardon; tont ee yui se rapporte à la fondation du convent de Kempten manque.

En 1843, la version de Reumont fut publiée en traduction suédoise dans les Folk-Sagor för Gamla och Unga ${ }^{4}$. Enfin, en 1845, P. J. Kiefer; dans mu onvrage intitulé Die Sagen des Rheinlandes, publia une version de notre conte ${ }^{5}$ apparentée de près à celle de Reumont, mais qui contient en même temjs quelıues traits primitifs (aItérés dans Reumont) qui peuvent faire supposer que la version de Kiefer a été inHuencée par celle de Crusins, simon par la version même de Birck. Voici quelques traits par lesquels cette version diffère de celle de Remmont:

Épisodes: (e) a Rome, Hildegarde se fait appeler Arabella; (f) Taland est frapyse de consomption; (g) exhorté par Hildegarde à se confesser devant nn prêtre, s'il veut vivre, il tait son erime envers Hillegarde $^{\natural}$; ce n'est qu'en monrant de saisissement, en alprenant qu'Hildegarde vit, qu'il se conlesse pleinement devant Charlemagne et Hildegarde: celle-ei fonde l'abbaye de Kempten, en reconnaissance de ce qu'elle est arrivée an bont de ses malheurs?

1 Pp. 259-il (la Table indique M. Friedheim eomme ayant recueilli cette version); 2:e éd. s. d. [1814], pp. 204-13 (Königin Hildegarlis). La version de Reumont a été réimprimée, vers 14\$3, par C. Trog daus son recneil de contes, ete. intitulé Rheinlands Wunderhorn (t. VII [s. d.]. pp. 136-49: Ilie Königin Hiletegardis).

2 Cp. la version de Sehreiber (p. 76).

. Cp. la version de Togt (p. 76).

- T. II. pp. 69-75 (Trottning Hillegardis).

${ }^{5}$ Pp. 210-8 (Königin Hildegard, tradition d'Ingelheim); s.e ed., Mayence 1866, arec le titre: I lie Sagen des Kheinlandes von Basel bis Rotterdam: 4:e éd., même titre, Iayence 18 í6. Il existe de cet ourrage des traductions françaises et anglaises: Légendes et traditions du Rhin de Bîle ì Rotterdam (v. la 3:e id., Mayence s. d. [1872]: La Reine Hildégarde, pp. 116-14; 1:ère èd., de 1847. Cologne, publiée sons le titre: Légendes et traditions du Rhin, traduites d'aprés le texte allemand par J. M. Dautzenberg; 6:e éd., Mayence 1892): The Legends of the Rhine from Basil to Rotterdam. translated by L. IT. Garnham (Najence Is6s; Queen Hildegard, pp. 106-14; 7:e éd., Mayence 1896).

* Cp. la version de Crusius (p. 70), qui suit ici sa source allemancle, tandis que Brusch a omis ce trait.

7 Trait primitif. Comme Kiefer renvoie aux annales de Kempten, il est évident qn'il a connu quel. que autre version dn conte que celle recueillie par lui. 


\section{C'IAPITRE IX}

\section{Conclusion}

Iyant passé en revue et groupé les versions que nons connaissons du conte de lu fromme chaste convoitie par son beun-fière, il convient d'embrasser d'un coup d'wil les principaux résultats acpuis.

Notre légende n'est pas en lapport génétique avec les antres contes oì l'héroüne est une femme injustement accusée par l'amant repoussé. Les tentatives que l'on a faites pour gronper les différentes versions de notre ligende ne penvent pas être considérées comme satisfaisantes; dans cet ouvrage on a tâché de retracer le développement historique de la légenule et d'en grouper les versions d'après leur état plus ou moins complet far rapport à la rersion primitive (ch. I). L'origine de notre légende doit être chercliée en Orient, probablement dans l'Inde (ch. II). Lioriginal indien de la légende n'a fas été retrouré, mais on connaît actuellement un assez grand nombre de versions orientales, faisant partie des grandes collections de contes, le Touti-Nameh, les Hille et une Nuits, l'Al Faray Ba.da Alsidda. Par les Wille et un Jours de Pétis le la Croix, notre conte a même eu une vogue étonnante en Enrope. Dans la branche orientale, les persécuteurs successifs de l'héroïne sont au nombre de quatre (ch. III). Déjà de bonne lemre, probahlement an $\mathrm{XI}^{\mathrm{e}}$ siècle de notre ère, une version orientale est venue se localiser en Europe, oil elle s'est divisée en deux branches principales, l'une garlant encore le nombre primitif de criminels (Gesta homanorum et Florence de Rome), l'autre ne ronservant que les deux premiers (Miracle de la Vierge). Dans sa forme européenne la plus complete, notre conte a tronvé sa place dans le chlèbre recueil de contes moralisés de la fin du XIII ou du commencement du XIV siècle, les Gesta Romanorum (clı. W). Apprarenté de près à cette version est le roman d'aventure français ell vers, artuellement jerilu, de la seconde moitié dlu X̃I e siècle, avec ses dérivés (ch. V). liatre grande branche occidentale est en premier lieu représentée par un miracle de la Vierye en latin du XII" siècle, qui a fait naître un très grand nombre de versions en diflérentes langues. Comme nons l'arous dijjà dit, les criminels ne sont que deux, mais il reste encore des traces évidentes du rôle du quatrième criminel de la version primitive (clı. VI). De la branche jurcédente s’est détachée très tôt, pent-être déjà ver's la fin dn $\mathrm{XI}^{\circ}$ siécle, nne branche caractéristiqne oì l'héroïne porte le nom de Cirscentia et où les persécuteurs de l'héroïne ne sont décidément plus que deux (ch. VII). Entin, vers la fin du $X V^{\text {e }}$ siècle un maître d'école allemand eut l'idée de mettre la piense légende, composée en l'honneur de la sainte Vierge, en raplort arec un person- 
nage historique bien connn, Hildegarde, épouse de Charlemagne. Il jugea cependant convenable de ne conserver qu un seul criminel, frère apocryphe du grand monarque. Cette branche tardive a montré beancoup de vitalité et a même eu la chance d“inspirer des effusions lyrico-épiques à quelqnes poètes morlernes, de talent secondiure, il est mai (ch. VIII) ${ }^{1}$.

\section{CHAPIT'RE X}

Table alphabitique des versions

Abrallam a Sancta Clara, Abrahamische Lauber-lliatt (Die gleiche Wiedervergeltung“, rers. d'Hildegarde) . . . . . . . . . . . . . 73

Acta Sanctorum, Apr. tom. III („De B. Hildegarde Regina, Campedonae in Sneria", par D. PАРEBRoch)

Al Farag Ba'da Alsidda, texte persan (Histoire de l'Arabe, de sa femme

Ouriya et de son frère) . . . . . . . . . . . . . 18

Al Farag Badda Alsidda, texte ture (Erviyyeh, la piense femme, que le frère

de son mari essaya de séduire) . . . . . . . . . . . . 18

Alphonse X (roi de Castille), Cántigas de Santa Mariu ("Como Santa María aindon a Emperadriz de Roma a sofrel-as grandes coitas per que passon") . . . . . . . . . . . . . . . . . . 54

Ancona (At. D'), Sacre Rappresentazioni (vers. de Cafrl) . . . . . . . 51, 1. 2 , même ourrage (vers. de Pulcl) . . . . . . . . . . . 51

${ }^{9}$ Le rapport des grandes branches de notre conte, d'après la classement adopté par nous, peut être représenté graphiquement de la maniòre suivante:

[Original inclien]

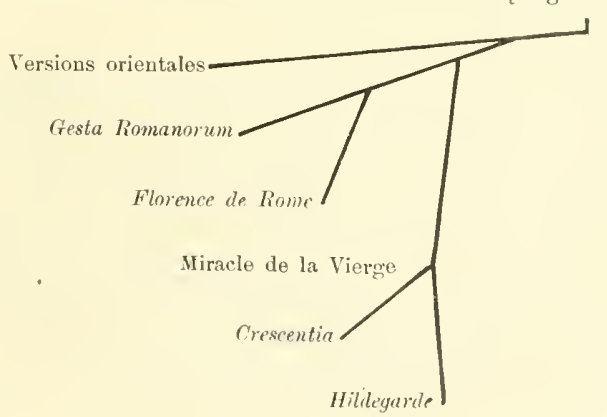


Axosis (Al. li), mime omrage (rés. de la vers. de Ferrari. par P. Mostr) 51, 11. 2

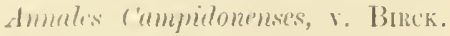

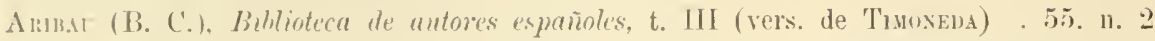
mime collertion, t. XVI; V. DrRas.

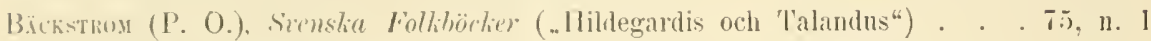

B.nnEL:Ta (G.), Sermones tam quadragesimales quam de sanctis (vers. du

Mirate de la Therge) . . . . . . . . . . . . . . 40

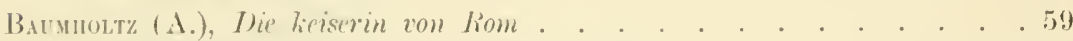

birschmib rnd Stioftung desz Fiirstlich Gottshauszes zu Kempten (trad. de la vers. de 13кus'li) . . . . . . . . . . . . . . . 70

Bescherhung rult Striftuny Dess Firrstichen Kloszters und Gottshauszes zue

Kimpten (trad. de la vers. de Bruscu) . . . . . . . . . . . 70. n. 4

Bererisi (Fr.). La Flura lmperatrire, rappresentazione per musica . . . 48

Biblioteca de autores españoles, v. Aribau.

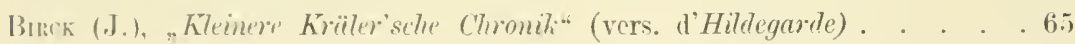

Bonser ('Tis. J.), dans Fabeln ans den Zeiten de\% Minnesinger ("Von dem

kaiser octaviauo dez wejb al\% vil laid umb ir kaüsch so daz si irs

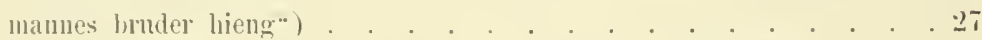

Businasi (A.). Istoria de Santu Gutielma fiola delo Re dangalterra et moglie delo he dungaria: etc. . . . . . . . . . . . . . . . . 49

Buxo (A.), Ia compassionenele hystoria de la beata Guiclma Reyina de Ongariu . . . . . . . . . . . . . . . . . 49

Botultí: (M:He), v. Rejsima.

Bragu. Jollstündige Sammlnny hilassischer und volkthümlicher deutscher Gedichte aus dem 15. mat 19. Iuhh., t. VI: v. Dietrich.

Bracid ('Tn.), Floresta de rarios Romances (vers. de Dias) . . . . . . 55

Buncelo (G.). La listoria di Flanen Imperatrie . . . . . . . . . . 47

- Flaria Imperatrice rappresentazione (?) . . . . . . . . . . 48, 12. 1

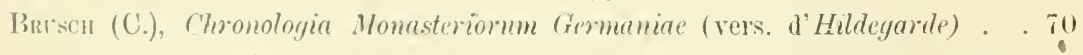

Buntus (Sin R. F.), A plain and literal translation of the Arabian Nights' Eintertainments, now entituled The Book of the Thousand Nights and a

Vight ("The Jewish Kazi and his pions Wife") . . . . . . . 14, n. 2)

- Supplementul Nights to the Bool of the Thousand Nights and a Night (.,The tale of the Devotee accused of lewdness") . . . . . . . 15, л. 5)

- 77er lionti of the Thonsand Nights and a Night, publié par L. C.

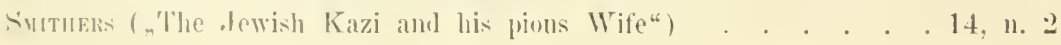


Burton (SIR R. F.), même ourrage ("The tale of the Devotee accused of lewlness") 15, 11. 5 Burton (Lady), ‥ Me Carthy.

Büsching (.. G.), Hans Sarhs (vers. de SAcus) . . . . . . . . . . 5s, 11. 1 (Le) Cabinet des Fíes, t. XV (vers. de Pétis de la Crolx) . . . . . . 19, 11. 1 Caffi (M.). Dell'Abbazia di Cliaramelle in Lombartia, illustrazione storienmommentale-epignafica (rés. de la vers. de Ferriri) . . . . . . 51

Caintigas de Santa Maria de ton Alfonso el Sabio, r. (Lis) Reat Acadenta espeñota. Clignett (J. A.) et Steenwinkel (J.). Spicgel Historiael of Rijmliponijli nen

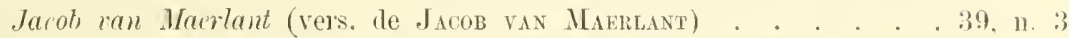
Cuodston (IV. A.), dans Originals and Analogues of some of chancer's Canterbury Tales („First Arabian Version“, = vers. Bonlac des Mille et une Nuits).

, même ourrage ("Second Arabian Tersion", = vers. Breslan des Mill et une Nuits) . . . . . . . . . . . . . . . . 15, 11.5 même ouvrage ("Ihind Arabian Version", = ver's. Montagne des Mille et une Nuits). —, même nurrage (vers. de Le Grand, trad.) . . . . . . . . . . 4і, и. 1 13. ก. 6 , même ouvrage (vers. de Vincest de Beauvals, trad.) 39

Colnci, v. Gattier de Colnci.

Colntios, v. Le Cointe.

Contes dévots, Fables ot Romans aneiens, v. LE Graxp.

Crusus (M.), Amnales Suevici (vers. d'Ilildeyarde) . . . . . . . . . T0)

Daniels (A. v.), Land- und Lhemecletmeh (vers, de Crescentia) . . . . (i1-2)

Dadtzenberg (J. M.), Légentes et traditions du Rhin de Bôle ì Rotterdam: v. Kiefer, Liggendes ete.

Destains (M.), Les Mille et une Nuits (. Aventures du Cadj et de sa femme") 13, 11. 7 Dias (B.), Historia da Imperatriz Porcina, mulher do Imperalor Lodonio de Roma, cm qual se trata como o dito Tmperador mandon matar esta semhora, etc.

Dick (IV.), Die Gesta Romanorum („De fratre imperatoris, qui concuphit imperatricem et suspendit eam vinam") . . . . . . . . . . 27

Diemer (J.), Die Kaiserrhronili nach der ï̈testen Handschrift des stiftes

Voran (vers. de ('rescentiu) . . . . . . . . . . . . . . (i1, n. 2

Dietrici (A.), Vollstündige Summlung kilassischer und rolhthïmlicher dentselier

Legonden aus dem 18. uml 19 Jahihundert (vers. de LaUn) . . . 78. 11. 2 Discipulus, v. Hehold. 
(Let) Llit de Flowrence de liomme, r. Jubisil.

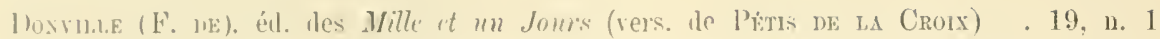

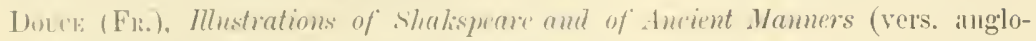

latime des Gestr Romanomm, trau.) . . . . . . . . . . . . 25, n. 1

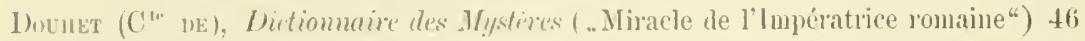

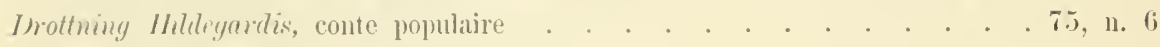

Denas (1.). Romancero general (vers. le Fuggo) . . . . . . . . . . 5́, 11. 3

Duras. (A.). daus l'Mistoire littéraire de la Frane, t. XIX (rés. de la rers.

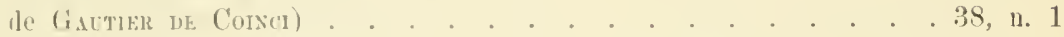

Eutak (B.), Discipulus retivims (,Imperatrici subvenit Maria in maximis angustiis“) . . . . . . . . . . . . . . . 40

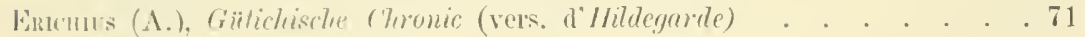

Farto (A. W.) Relationes curiosne Bararicae („Sieglrangende Unsehuld Hilde-

gardis") . . . . . . . . . . . . . . . . 72

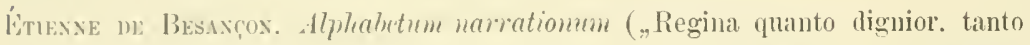
magis castitatem sum servare debet". vers. du Maracle de la Fierge) 4:

—, trał. aliglaise dı même ourage . . . . . . . . . . . . . . 42--3

, trad. catalane du mime ourraye, v. Recull ete.

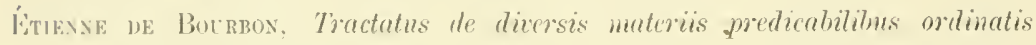
rt distinctis in spptem partes secundum septem dona spivitus Sancti etc.

(vers. dn Mriacle de la Vierge) . . . . . . . . . . . . . 42

Fabeln ans den Ziten der Mimesinger; r. Bodmer.

Faiz et miracles de Nostre Dame („De la femme d'un empereur qui pour

bien fere eut a sonftrir moult de manlx, " ete.) . . . . . . . . 36

Fenuma (A.). Yita di Sonta Guglielna . . . . . . . . . . . . . 50-1

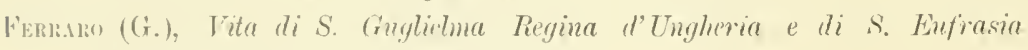
vergine Romana (vers. de Bonfantw) . . . . . . . . . . . 49

(La) Fista di Santu Gnylielma, v. Pusci.

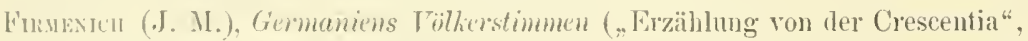

vers. de (Deci) selen troist) . . . . . . . . . . . . . 57, 1. 4

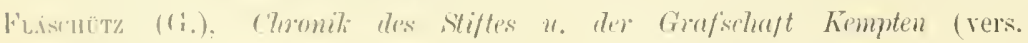

d'Hitrlegerde) . . . . . . . . . . . . . . . . . . (55. 11. 3

Flaver merratice, v. Buscrio.

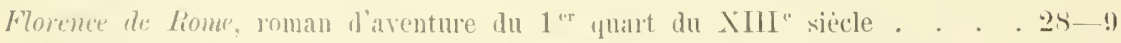

(Le bene) Florenere of Reme, v. Rıтsos, Viëtor.

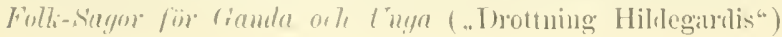


Frantz (J. J.), Mistoria Caroli Magni (vers. d'Mildegardi)

Friedheim (M.), v. Reunont.

Frischun (J.), Hildegardis Magna, Eine schöne comedien rme Fran Hilde-

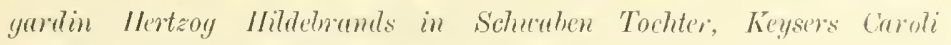
Magni Gemahlin . . . . . . . . . . . . . . . . . 74

Frischum (N.), Mildegradis magna . . . . . . . . . . . . . . 74

Operornm poeticorum pars scenicu (., Hildegardis magna“) . . . . . 74, n. 22

Fuego (J. M. IEL), La peregrina doetora (vers. du Mivacle de la Tierge) . j(;

Funnivall (F. J.), dans Originals and Analognes of some of Chaucer's Can-

terbury Tales (.The Tale of the Wife of Merelaus the Emperor"*) . . 25, n. 4 Hoccleve's Works ("Jereslans Wife") . . . . . . . . . . . 26, n. .)

Garlandia, v. Johannes de Garlandia.

Garmham (L. Mr.), v. Kiefer, The Legends etc.

Gautier de Conci, Wiracles de Notre-i)ame (De l'Empereri qui garda sa chastée par monlt temptacions"; etc. etc.); ep. Míon . . . . . . 37

Gauttier (Éo.), Les Mille et me Nuits ("Aventures d'un Cadi et de sal femme“) 1:3, n. 7 GEIB (K.), Die Sagen und Geschichten des Rheinlandes (Die Königin

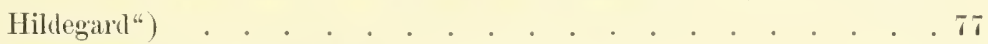

Die Volksagen des Rheinlandes („Hildegard“) . . . . . . . . 77

Genthe (F. W.), Dentsche Dichtungen des Miltelalters (rés. de la vers. de

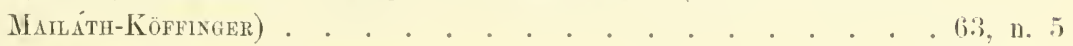

Gesta Romanorm, rersion anglo-latine . . . . . . . . . . . . . 25

, version latine continentale (De Octariano qui super omnia uxorem

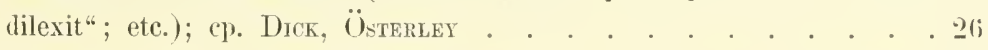

, trad. anglaise ("Merelans the Emperom"); cp. Furnivalu, Herrtage,

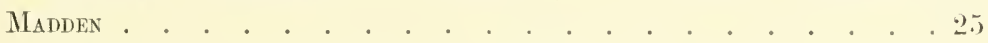

, trad. allemande ("Von dem chaiser Ottaniano dez weil als vil vmb ir chensch led so daz sey . . . man pruder hing“); cp. Bonmer, (irässe, $M_{\text {assuani }} \cdot$. . . . . . . . . . . . . . . . . . . 27, 110

: containing Fifty Eiyht Remarkable Histories, Collected originally from the best and most ancient Roman Records. - - By A. B. . . . . 206, 11. 3 : or Fifty-eight Ilistories Originally (as 'tis said) collerted from the Roman Records. - - By B. P. . . . . . . . . . . 26, 11. :3 (II) Giarlino d'essempi (?) . . . . . . . . . . . . . . . . . . 47, n. 1

Goedeke (K.), Deutsche Diehtung im Mittelalter (lés. des velsions de Mañ́thKöfFinger et de la kiaserchromili) 63. 11. 5 


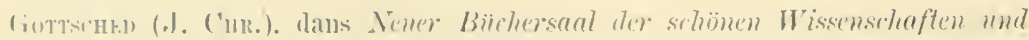

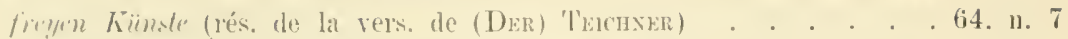

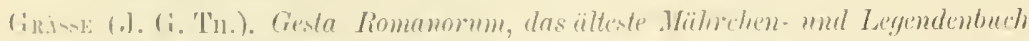
des christlichen Mittlallers (. Vom Kajser Octaviauns") . . . . . 27

Cimun (11.), dans la Leipziger Litcratur-Zeitumy, anmée $1 \mathrm{~s} 1 \mathrm{~s}$ (fragm. de la

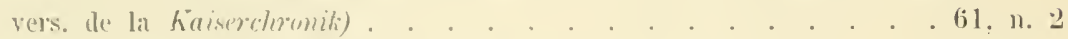

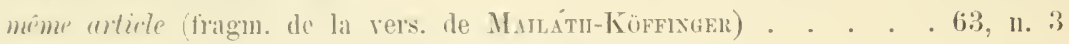

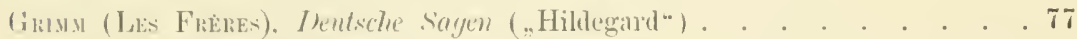

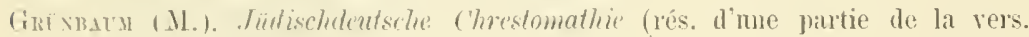

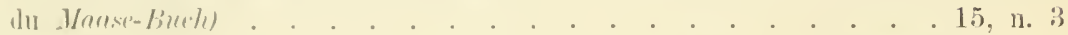

Haburit (M.) et Flatsoner (II. L.). él. arabe des Mille el une Nuits (Yers. Breslau: La piense Femme accusée le libertinage) . . . . . 15

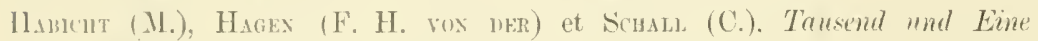
Nacht (-Abentener eines Kadi"s und semej Fran“) . . . . . . 13, п. 7 H.wix (f'. H. ros usk), (iestmmtnlentener („Crescentia“) . . . . . . . (63 mum omragk (rés. de lit rers. précédente). . . . . . . . . . 63.3. 11. 4 Tausend mul rin 'Tay (.. Geschichte Repszima's") . . . . . . . . . 19, n. 2

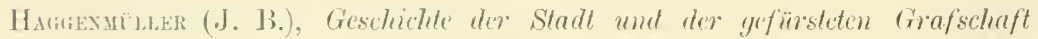
kemplen (vers. d'Hilleyarde) . . . . . . . . . . . . . TI

Hsix (.J. (i. vox), Griechische uml albanesische Mïrolen (. Ton der Frau, die (iutes thut nud Undank esfährt") . . . . . . . . . . . 2.2

Hamer (J. rus), Y. Zinserlisg.

Harpes (F. W.), Grösacste Denlibirdiglieiten der Ilelt oder so genundte Relationes rurinate (.. Die listige Kenscheit", vers. d'/lidegarde) . . . . T:2

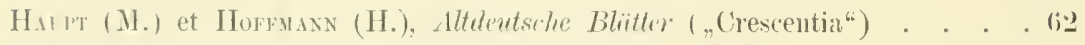

Heisz: (K T.). dans Idmma und Ilemode, t. I (fragms. de lat vers. de la Kaiserdironiti) . . . . . . . . . . . . . . . . (i1, n. 2

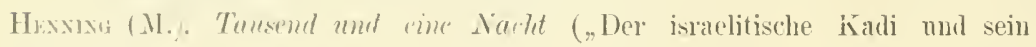
frommes $W_{\text {eib") }}$. . . . . . . . . . . . . . . 14, n. 2

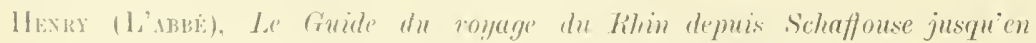

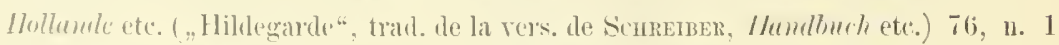

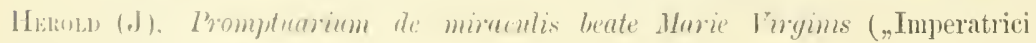
subenit Maria in maxinis angnstiis" . . . . . . . . . . . 40

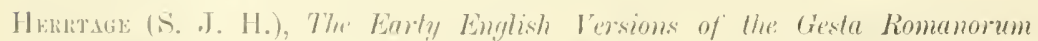

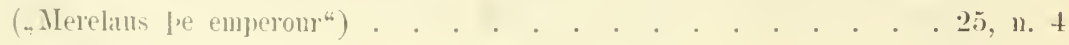


Hinricus (G.), Kleinere Schriften mon Willelm Grimm; v. IV. Grum.

Histoire litteraire de la Fremer, t. XIX; v. Duval; - t. XXVI; v. P. Paris.

Histoire littóraire des Fimmes Francoises, v. In Porte.

(1,a) Historia della Serenissima Regina di Polonia, laquale due volte iniquamente fu mandata nelle silve ad uccidere, etc. . . . . . . . . 51

(En lustigh och sanfärligh) Historia om Drottming Hildegardis, Caroli Magni Hufru, och hennes Styfflbroder Talando . . . . . . . . . . 75

(En underbar) Historia om den stöna Repsime . . . . . . . . . . 19, n. 3

Hoccheve (Tr.), Fabula de quadam Imperatrice Romuna . . . . . . . 26

Hüber (F.), Tusterhliche Gedächtur's dev nortrefflichen Geschidhten, Heiligen

Stiftungen ond umedertichen stands Veränderungen _ - _- (vers.

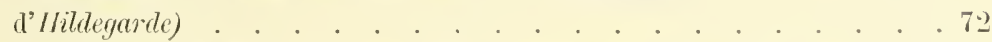

Hunbert de Rourss, Lither de abmolantia exemplorum (vers. du Miracle de

la Vierge . . . . . . . . . . . . . . . . . . . . 42

(Ein schone und warwaftige) hystori ron cimer Kayserin zx Rom: genandt

Crescentia: gar kurtaweylig zulesen. . . . . . . . . . . 64

Idunna und Hermode. v. Henze.

(L') Innocenza svelata in Santa Gnglielmu, ridotta in prosa — - - da P. G. S. 5.

Istoria di Flavia Imperatrice, v. Briccio.

Istoria di Santa Guglielma, v. Bonfadinı, Boxo.

JaCob van MaErlant, Spregel Historiael ("Van der keyserimen van Rome“) . 3!)

Jasander, Der in fremden Ländern sich lang aufgehaltene, numberro aber vieder angelangte Tentsche Ilistorten-Srlwaber, - - - - - (vers.

d'Hildegarde) . . . . . . . . . . . . . . . . . 7:3-4

JEAN DU (on DE) Vrasar, Niroiv historzal (De lempriere de laquelle elle [la mere dien] garila la chastete et deffendit quelle ne fut corrompne ne violee de ses mamuis serfs"; _ _ - . . . . . . . . . 39

Johandes de Garlandis, Stelle maris de Miraculis Beate Marie Virginis (rers.

du Mivacle de la Vierge) . . . . . . . . . . . . . . . 43

Johannes Gobir (on Junion), Scala reli (vers. In Miracle de la Tierge) . . 39-40) Johannes Herold, v. Herold.

Johannes Major, Mngnum Speculum Eremplomm („Beata virgo Maria Imperatricem castissimam, marito fidelissimam, \& propter corporalem pulchritudinem multa perpessam à tribulationibus liberat") . . . . . . . $4(1)-1$

Jubinal (A.), Nomean recueil de Contes, Dits, Fablianx et autres pièces ini-

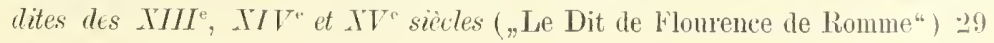




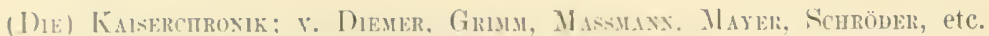
Liniserin ron rom revtriblen (?)

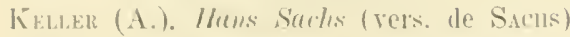
54

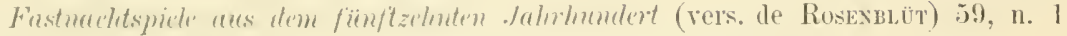

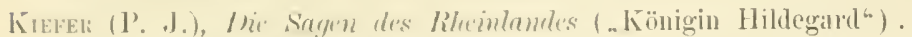
79

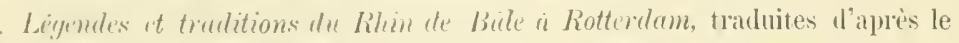
texte allemand par .J. M. D.ı T\%rsmbla (.La Reine Hillégarle“) . . 79, n. 5) - The Le'gents of the Rhime from biesil to Rotterdam, translated by L.

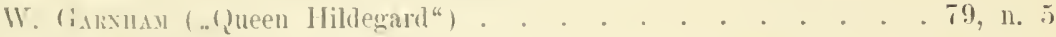
Kivi, The I'rsiun and the Thurkste Tales (..'The Story of Repsima“) . . . 19, n. 2 Kircunof (H. IV.). W'oul-Cmmuth (. Ton könig Carolo magno eine ware histori") .

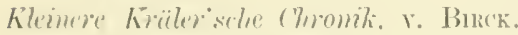

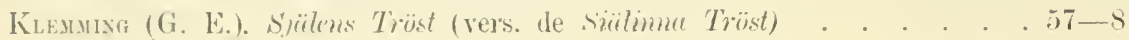

KRÄLER (J.), V. BIRCK

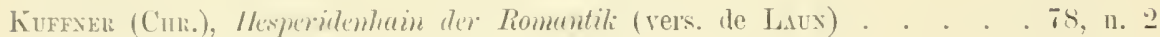
(Jer) kinige broch Ninuer $\hat{e}, r$. Dariets.

Kürsilser (J.), Deutsche Nationul-Litteratur, t. II; v. PIPER.

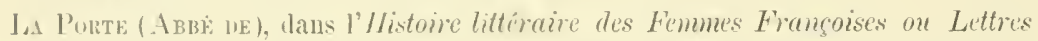

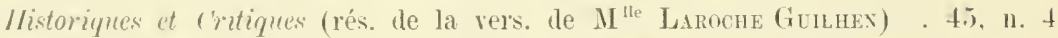

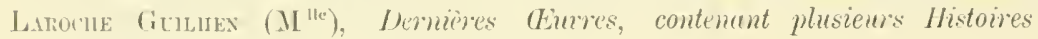
galantes; etc. (..Adeleide Reyne de Hongrie*) . . . . . . . . 4i

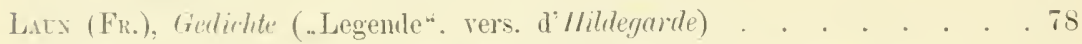

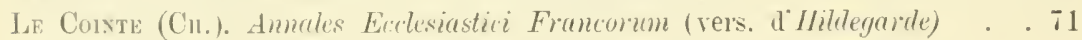
Lecor de la Marme (.1.). Anedotes historiques, légendes et apologues tirés

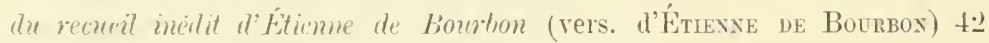
Leggemla di Santa Ginglielmu, ete., r. Bosfadixi, Boxo.

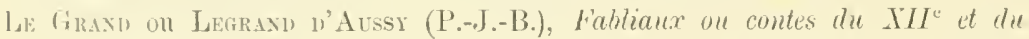
$T I I^{\mathrm{e}}$ siecle (..De la bonne Impératrice qui garda loyalement la foi du mariage, on ile l'Emperen de Rome qui fit le royage d'ontre-mer*) . t5

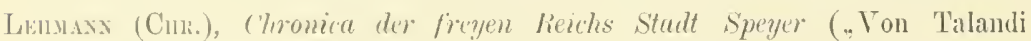
Künigs Caroli mehelichen Bruders grosser Untren, so er dem König und seinem (iemahl Hillegart erwiesen") . . . . . . . . . . 71

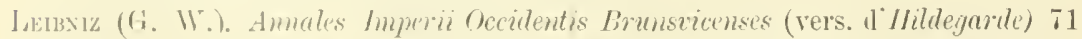

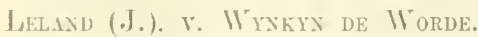

Liber de almulantia exemplormen, v. Hombert dE Rosans. 
Lorseleur-Deslongchamps (A.), v. Panthéon littéraire.

Liser (J. P.). Abendlündische Tansend mal eine Nacht (vers. de Crescentia) 63

Mase-b'uch (rers. orientale) . . . . . . . . . . . . . . . 15

II Cartur (J. H.), Lady Burton's Edition of 7er Mhsbamd's Arabian

Nights (.The Jewish Kazi and his pious Wife") . . . . . . . 14, 11. 2

The Thousand and One Days (,The Story of Repsima") . . . . . 19, n. 2

Madoen (SIr F.), The old englishe rersion of the Gesta Romanorum ("Mere-

lans the Emperou" ${ }^{*}$. . . . . . . . . . . . . 25

Maerlant, V. Jacob van Maerlant.

Magmm Speenlum Exemplorum. v. JoHanses Major.

M апда́ти (J. N.), Auserlesene altuentsehe Gediehte („Crescentia“) . . . . . 63

Manı́́th (J. N.) et Köffivger (.J. P.), Koloczaer Codex altilentscher Gedichete

(vers. de Crescentia) . . . . . . . . . . . . . . 63

Mariale, $\mathbf{}$. Miracles de la Vierye.

Marmer (X.), Contes populaires de differents pays ("La Vertu d'une femme") 12

Mantixus von Cochem, Auszerlesenes Iistory-Buch, éd. 1687 („Wie die Mutter

Gottes einer Keyserin in ihren grossen Noethen zn Hülff kommen“) . 41

, même ouvrage, éd. 1732 (.,Von der unschuldigen Beklagung, langwürdigen

Elend, und endlicher Erhöhmng der seligen Kayserin Hildegardis“) . 72-3

, Hildegardis, die Heitige, Gemahlin des Laisess Karl des Grossen, zueinal unselneldig zum Tode verurtheilt — — . . . . . . . . . . 73, 1. 1

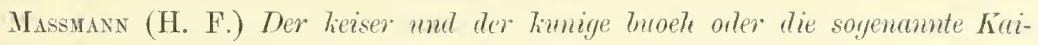
serelinonit ("Narcissus") . . . . . . . . . . . . 61, n. 2 , même onvrage (rés. de la vers. précédente: "Narcissus oder Crescentia") . 61, n. "2) même ouvrage (, Von octaviano dem chaiser des fraw so vil vmb ir rainigchait laid so dz sy irs manns brider vieng und in darnach wid' ausz liesz") . . . . . . . . . . . . . . . . . 27

, même ouvrage (ver's. de Vincent de Beauvais) . . . . . . . . 39

, Der liünige buoch Nimer $\hat{e}$; v. Daniets.

MaYer (J. M.), Der Kaiser und der Kömige Buch oder die sogenumbe Kaiserchronik ("Narcissus") . . . . . . . . . . . . . 61, n. 2

Megeridn (H. U.), v. Abramair a Sineta Ctara.

Megrim (M. J.), Ailff omd Nideryang dess Fiirstlichen stieffts vndd Gottshäisz Kempten (vers, d'Ihidegarde) . . . . . . . . . . . . 71

Mion (M.), Nonvean reeneil de fallinna th eontes inódits (vers. de Gautuer DE (oIncr) 
Whi.ot (J.), Mrrudes de Vistre Dame (..Dune grant dame de Rounme qui fint par ilen foiz faulsement acrisse de aldultere et de omicide. la quelle par la grace de la vierge Marie, a qui elle recumroit, fut deliuree et preseruce $\left.{ }^{*}\right)$.

(Les) Melle et un Jomrs, r. PÉTIS le l.A C'ROIX.

(Le's) Mulle it me Nuits, ms. Wortley Montagne (Le Cadi de Bagdad, sa vertnense femme et son méchant frère). . . . . . . . . . . 13 érlitions arabes de Bonlac. de C'alcutta, de Bombay. ln Caire et de Buyrouth (Le Cauli juif et sa piense femme) . . . . . . . . 14 íl. arabe de Breslau; r. Hanicut-Fleischer.

, traluctions de la vers. Bonlac; r. Bertos, Clocstos. Hamer, Hexing. M" C.nthy, Paye, Thíbutiex, Weil. Znserling.

, truductions de la vers. Breslan; r. Buntos, Crouston, Parne.

- traductions de la vers. Montague; v. Destains, Gadttier, Hagex-HAвтент-S̈нацL, Sсотт.

Mirates de la Tierge, mss. latins (vers. A: „De pudicitia et tolerantia cuinsdam imperatricis" ${ }^{6}$ ete. ete.) . . . . . . . . . . . . . 3t

, ms. latin (vers. B. = la vers. de Tincext de Beaurais) . . . . . 34

, 11к. latin (vers. C) . . . . . . . . . . . . . . . . . . . . . . . . . .

—, mss. latins (vers. D: . De imperatrice miraculmu“) . . . . . . . . 35

. mss. français, en prose (..De Nostre Dame, qui garda la femme de

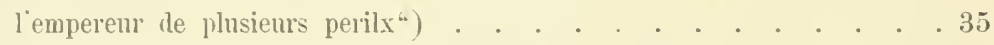

- ms. français, en mose; r. Faiz et miracles de Nostre Dame.

. ms. français, en vers ( .De la sainte empereris qui garissoit les lieprons") 36

Miracoli della Halonna (?) . . . . . . . . . . . . . . . . . . . . 4 . .

(I)ic) miraculen van onse lieue romue (,Van eeme keiserinnen") . . . . 36

Moneste lo S. Alable, La Jonarchie Sainte. historique, chronolorjique et

génealogiqne de France (vers. d'Hilelegarde) . . . . . . . . . 71

MoIrs (J.), v. Sultze.

Moxmerque (L.-o.-N.) et Mremer (Fr.), Theütie Frangais an Moyen Age ( Miracle de Nostre-Dame, de lempereris de Romme“ etc.) . . . . 46

Muxti (P.). Y. D'Axcoxa, rés. de la vers. de Ferrari.

Morayta bet sagrario (M.), dans la Rerista politica, filolosófica y literaria titulala La Riazom, année 1850 (rés. de la vers. l'Alphosse X) . . 5t, n. 3 - dans le Boletin biblingrafico español. t. IV (rés. de la vers. l'Atprosse X) 54, u. :3 Moser (J. J.), Schecibische Chroniti (trad. de la vers. de Crusits) . • . . 71 
Musarra (C.). Santa Flaria, mupresentazione sacra (?).

Missafa (A.), Eine altspanische Prosedaratellung der chesentiasenge („Muy fermoso cnento de una santa emperatriz que ovo en Riroma et de sul (astidat" ") :S , mêne article (fragments de la vers. d'Alphosse X) . . . . . 5t, n. 3 , Über eine italienische metrische Intesteflung der cressentiastye („Del ducha d'Angio et de Costanza so mojer") . . . . . . . . . . 53

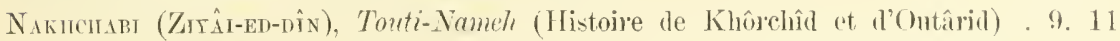
, même ouvrage, trad. franç. („De la rare beauté de Courchide, et des malheurs qui lui surviment par l'effet de sa beanté") . . . . . . 11. 11. 1

Nener Büchersaal der schönen Wissenschaften and freyen Kümste, v. Gorrsoned.

Nrso (A. de), Usi e costumi abruzzesi (.Favola gentile“) . . . . . . . 52

Norelle d'ineerti autori del secolo $\mathrm{XT}^{\circ}$ ( „Storia d'ma doma teutata dal cogmato, scampata da pericoli, ritornata in grazia per sua castità e livozione") 53-4

Oichete. v. Hoccleve.

Originals and Analogues of some of Chanter's Canterlury Tales, v. Cuoustox, Fornivall.

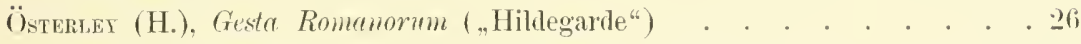

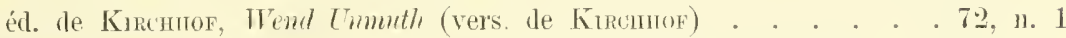
Panthéon littéraire, Contes orientanx, t. II (vers. de PèтIs ne la Cronx) . . 19, 11. 1 Papebrocir (D.). v. Acta Śmatornm.

P.rals (G.) et Robert (U.), Mirucles de Nostie Dame par personnages (., Miracle de l'Empereris de Romme") . . . . . . . . . . . . 46

PARIs (P.). dans l'Histoire litteraire de la France. t. XXVI (Jés, de lia vers. du Roman de Florence de Rome) . . . . . . . . . . . . 30, n. 3 Passero (F.), L'Urania orero La Costente Ioma . . . . . . . . . 46 Paumoltz, v. Baбmholtz.

Payne (J.), The Booli of the Thousend Nights and One Night ("The Jewish Carli and his Pions Wife") . . . . . . . . . . . 14, 11. 2 , Tales from the Aratic ("Story of the Pions Woman acensed of Lewduess") 15, n. 5 (The) Persian Tales, or The Thousand and One Days ("The Story of Repsima") 19, n. 2 Pertsen (II.), dans Zeitschr. der Dentschen morgentëndischen Gesellschaft, t.

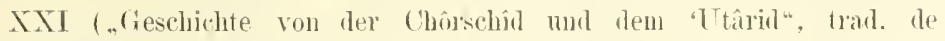
ХАКнснаві) . . . . . . . . . . . . . . . . . . . 11

Púts le la Cromx (Fr.), Ville el un Jours (.,Histoire de Repsima“) . . . 19

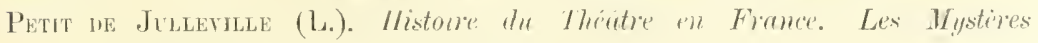
(„L'Impératrice de Rome“. anal.) . . . . . . . . . . . . thi 


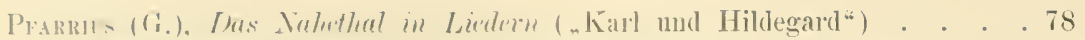
kurlmum (?) . . . . . . . . . . . . . . . . 78, 11. .

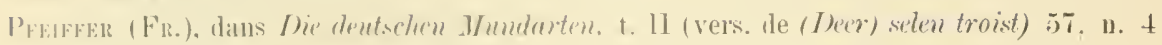

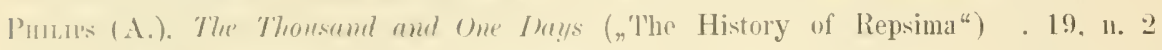

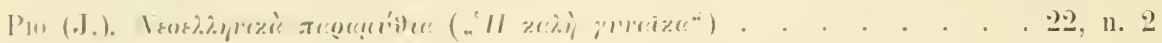

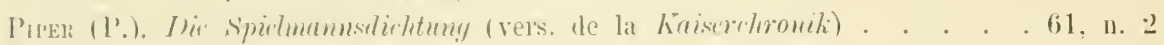

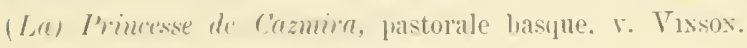

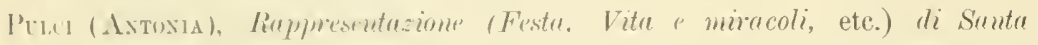
Gimplirma . . . . . . . . . . . . . . 51

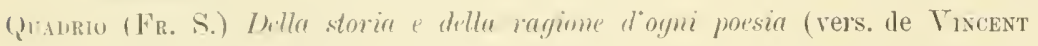
le Beatrilis, trarl.) . . . . . . . . . . . . . . . 39

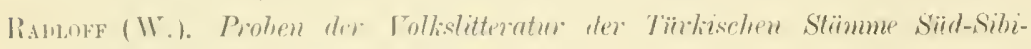
rims (. Das Weils als Fürst") . . . . . . . . . . . . . 16

Liapprasentazione (delle Vita et Miracoli) di Sunta Guglielua, v. Prber.

Razzi (s.), Miracoli della gloviosa Vergine Maria nostra Signora (,Maria souniene al rna Imperatrice posta in granissime angustie“) . . . . 41

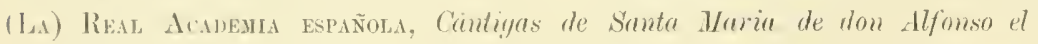
Sútrio (vers. d'Alplonse X) . . . . . . . . . . . . 5t mone ourrage (rés. de la vers. précérlente) . . . . . . . . 54. 11. 3

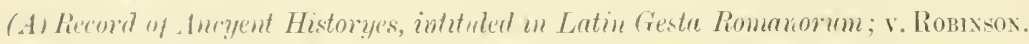

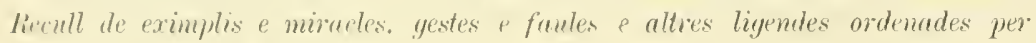
A-li-C: (, Miracle que la verge Maria fen a Lemperadriu muller del Emperalor de Roma") . . . . . . . . . . . . . . . . . 43

Rerser (K.), Sugen, Golwrïuche de Sprichnörter des Allyüus (., Hildegard mil 'Taland" ${ }^{6}$ ) . . . . . . . . . . . . . . . 65

Pit planiscle (Repyonische) ('hromit, v. ITELAND.

Repsimu (conte populaire suédois) . . . . . . . . . . . . . . . 19, 1. 3

essai d'nue tragédie domestique [par M $\mathrm{M}^{\text {lle }}$ Boumlè $]$. . . . . . . 21

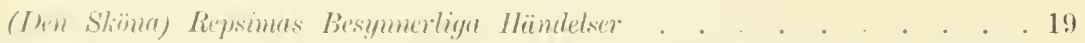

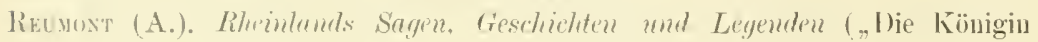
Hililegardis") . . . . . . . . . . . . . . . . . 79

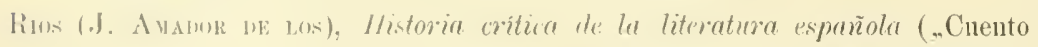
miny fermoso del enprador (ottas de Roma, et le la infante Florençia

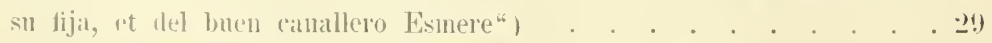

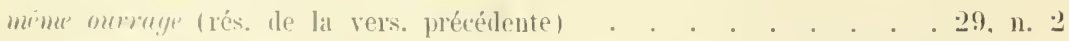

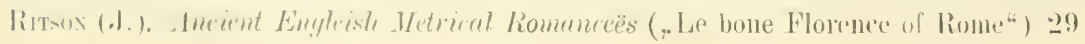


Robinson (R.), A Recorel of Ancyent Historyes. intituled in Latin Gestre liomunovum (vers. des Gesta Romanorum) . . . . . . . . . . . 20;

Roman de Florence de Rome, roman d'aventure de la 1 ìre mroitié in XTV siècle s? Roman Records, v. Gesta Romanornm, Roвinson.

Rosen (G.). Tuti-Nameh ("Geschichte der Merhûma“) . . . . . . . . 12

Rosenbü̈t (H.), Een liepleich history von groszer sehone. gedult uml hinsingit einer edeln keyseryn; etc. . . . . . . . . . . . . . . . 50-!)

Eyne schone lugstorie wan eynem lieyszer to rome mule simer erlaken Koyse-

rinne wo de myt groter rulscheit belayen wart (trad. de lis vers. précédente) 5!)

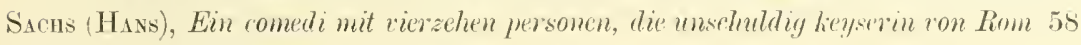

Süchsische Weltelroniti, r. W'EILAND.

Scale veli, v. JoHannes Giobir.

Sceltu di curiositì letteraric inerlite o rare dal secolo XIII al XTII, no. 15!);

v. Ferraro.

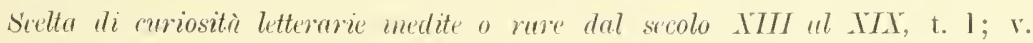

Novelle dincerti antori etc.

Schade (O.), Crescentia. Ein niderrheiniscles Gedicht uns dent arbölften Jahrlewndert . . . . . . . . . . . . . . . (i1, n. .

Sснӧнитн (O. F. H.), Historie von der gerlulligen Königin Crescentia . . 63

Schöppner (A.), sagentuch dev Bayerischen Lande („Hildegardis und Taland”.

vers. de $\mathrm{LAUX}_{\mathrm{AUN}}$. . . . . . . . . . . . . . . . 78, 1."

Schrember (A. W.), Handhuch fïr Reisende am Rhein ron Srhafhausen bis Ilolland ("Hildegard"); c"p. Henry . . . . . . . . . . . . 76

, Anleitung auf die niitzlichste und yemssiollste Art den Khein - _ - $z$ bereisen ("Hildegard") . . . . . . . . . . . . . . . 7 (i. n. 1

- Auswall der interessantesten Sagen aus den Gegenden des Fiheins und des Schuarzwaldes (.,Hildegard“) . . . . . . . . . . . . . 76

- Volkssagen ans den Gegenden am Riliein und am Tamme (.Hildegard") 76, 11. 1

__, Sagen aus den Rheingegenden, dem Srhwarawalde und den Togesen („Hildegard") . . . . . . . . . . . . . . . . . . 76, n. ²

-_. Traditions populaires lu Rhin, de la Forêt Noire. de la Jallée du

Nècre, de la Moselle et du Taums ( Hillegard".) . . . . . . . 76, n. .2

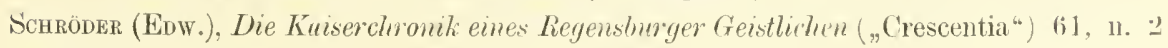
Schulz (Fr. A.), v. Laun.

Scoтt (J.), Arabzan Nights Entertainments ( Allventures of the Cauzee, his Wife etc.") 


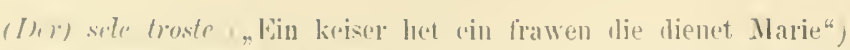

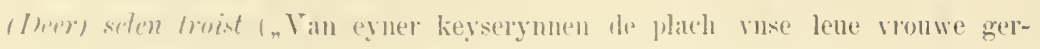

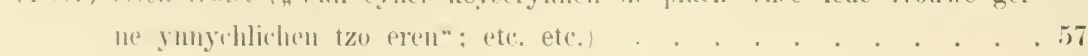

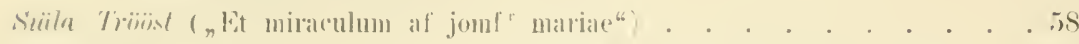

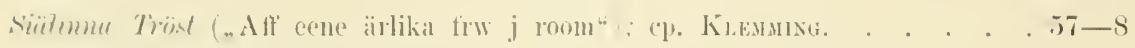

(Iha) sichn tromst o Hoe die keyserinne tweewerf met loghen ter doot vordeelt

Was. embe hoo se onse liene vronwe matra v'loste"). . . . . . . 5

Sprenlum cormploram (... Castitatis nerito imperatrix per Beatam Tirginem ex multis angustiis liberatum . . . . . . . . . . . . . . . . fo

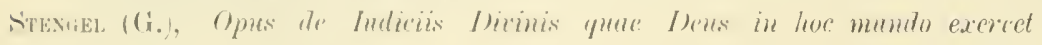

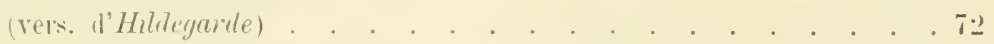

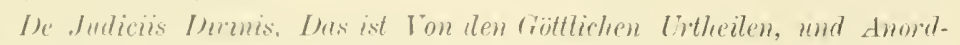

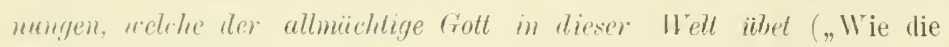

Inklag der hatyserin Hildegardis auff len Urheber gefallen“) . . . 72. n. 2-

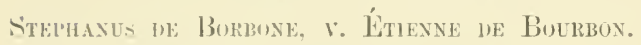

SiLt\%e (.J. Mo[Rs), I. (Iteer) selen troist.

simas (C'r.). Geste liomanorm (vers. de Robison) . . . . . . . . . . 26

Tansend und ein Tay (..Historie von der liepsima“) . . . . . . . . . 19, n. 2

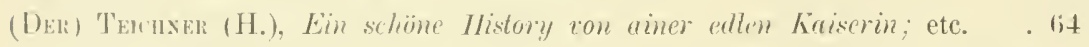

'T'uen ( N.), Traditions allemandes recueillies et publices par les Froves Grimm

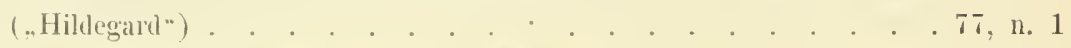

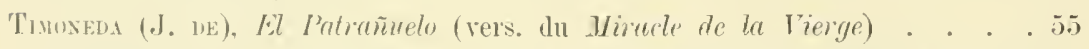

T'on(n)asbla (F.). La Deuotissima, Rapp). de Santa Gulielma liegina d'In-

yuria: etc. . . . . . . . . . . . . . . . 52

Tomti-Numel, texte persan; v. NaKn'навт.

-, texte ture (Histoire de Merhûma); ç. Rosen, Wlekerhauseli . . . 11-2

Timalions joupulaires du Rlim. etc.; v. Schrember.

'l'mintmix (G.-S.), Contes millits des Mille et me Nuits ("La Vertueuse

Istálite") . . . . . . . . . . . . . . 14, n. 2

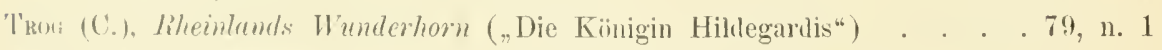

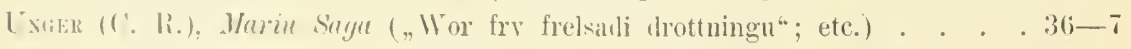

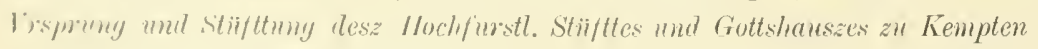

(vers. de Butscn, trad.) . . . . . . . . . . . . 70, n. 4

Tir der Yeres (,Du roy yui ala outremer“; etc. etc.) . . . . . . . . 44

Vilitor (II.), Le tone Flovence of Rome . . . . . . . . . . . . . 29

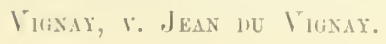


Trancent de Beadrars. Speculum listoriale (.,De Imperatrice cuins castitatem

[Beata Virgo] a violentia servornm eripmit; - - - . . . . . 3s-?

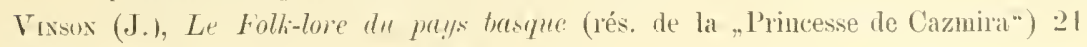

Vita e Mracoli di Santa Guglielma. v. Pulci.

Yogt (N.), Rheimische Geschichten und Sayen (vers. d'hildegarde) . . . . Tf

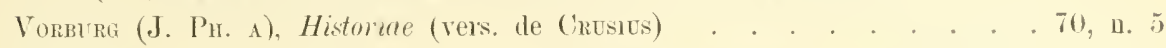

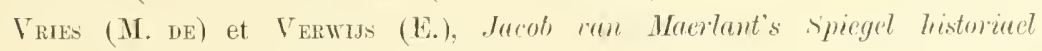

(vers. de Jасов vin Makrlant) . . . . . . . . . . . . 3!, 11. 3

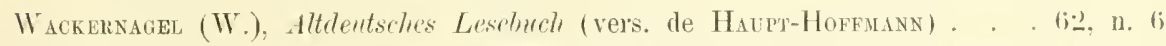

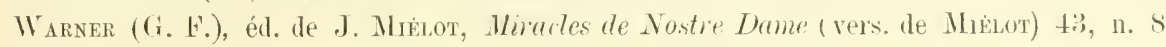

Weber (H.), Tales of the East (,The History of Repsima“) . . . . . . 19, n. 2-

WenL (G.), Tausend und eine Nacht („Die tugendhafte Fran eines israeliti-

schen Richters") . . . . . . . . . . . . . . . 14, n. 2

WeIland (L.), sächsische W'eltchroniti (vers. de crescentia) . . . . . . 62

Wickerhadser (M.), Die I'mpageimärchen ,Die kensche Merhmma") . . 12

, Die dreissig Nächte (? Histoire de Merhn̂ma) . . . . . . . . 12, 11.4

WolfF (O. L. B.), Poetischer Ilansschatz̃ des dentschen lollies (vers. de Liun) 7s, n. 2 , même ouvrage (vers. de Pfarrius) . . . . . . . . . . . 78, 1.5

Wrnkyn de Worde, Gesta Romanomum (vers. des Gesta Romanomm) . . . 2.)

Zinsering (A. E., Der Tausend und Eimen Nacht noch nicht aiborsetzte

Mährehen, Erählungen und Anchototen ("Die tugendhatte Israelitin“) 14. 



\title{
Appendice
}

\author{
A \\ Version du Maase-Buch, chap. 203 (éd. de Wilmersdorf); traduction.
}

Un homme. voulant se rendre dans des pays étrangers pour cause d'achats, confia sa femme, pendant son absence, aux soins de son frère. Celui-ci la reçut avec la promesse de la garder comme le ferait un frère, et le mari partit le cemr léger. Le frère installa sa bellesteur dans un étage de sa maison et lui donna des domestiques a part, de sorte qu'elle ne manquait de rien. Le frère lui-même se chargea du lốf d’intenclant, pour que rien ne mancquât. Tu jour qu’il allait derechef voir sa belle-søen pour s'enquẻrir de sa santé et de ses désirs, il fut saisi de l'envie d'induire sa belle-surur, qui était d'une grande beauté, à un arte malhonnête; mais elle, en femme piense, reponssa sa proposition, disant que ce serait un péché envers Dien et son mari, et elle lui rappela le dixième commandement: Tu ne convoiteras point la fimme de ton prochain, ce qui entraîne la punition de la lèpre.

Il se contenta silenciensement de son refus. Un jour qu'il était de nouveau chez elle, ce terrible désir s'empara derechef de lni, et, par prudence, il éloigna de la maison les domestiques. Comme elle refusait encore de se prêter à ce ru’il vonlait, it essaya de la prendre de force. Ellte se mit à crier très fort, mais personne ne l'entendit, puisque les domestiques n’etaient pas à la maison. Elle rẻussit pourtaut, en $y$ mettant tontes ses forces physiques, à l'empêcher dans son projet infâme.

Furieux de cet échec, il acheta denx fanx témoins qui devaient déclarer qu'ils avaient aperçu la lenme en commerce charnel avee un des valets. ('ette terrible déposition eut pour résultat qu'elle fut condamnée par la hante cour à etre lapidée, et cet arrêt fut exécuté.

Or, le troisième jour après cette exécntion, an soir, un étranger, „ni venait avec son fils de pays lointains, en ronte pour Jérusalem, afiu de faire donner à son fils, dans cette place sainte, une bome instruction, s'assit pres du monceau de pierres, sans savoir que ce moncean provenait d'une lapidation. Alors ils entendirent une voix qui sortait de dessons les pierres ef „ui disait: „On m’a lapidée sur un faux tẻmoignage“. Ils éloignèrent les pierres et virent la f'mme encore vivaute. La femme lenr laconta son aventure; ensuita elle demanda oi l'homme allait avee son tils. Le père répondit: „A Jérusalem, pour que mon fils prisse s'y adonner à 


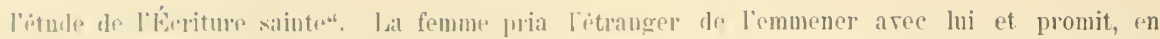

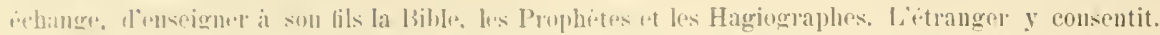

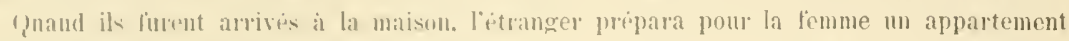

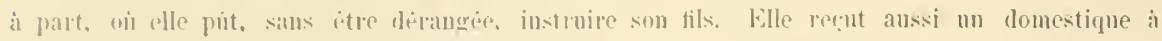
elle, qui lui apportait is manger et ì boire.

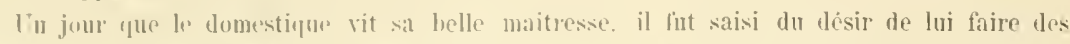
propositions malbounites. Mais elle les repoussal ènerginuenunt et lui tit observer qu'elle itait

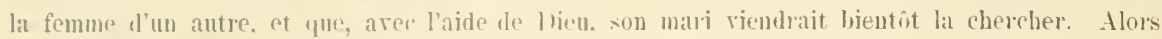

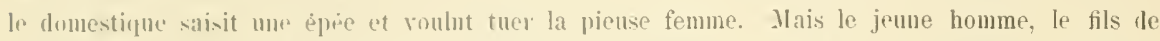

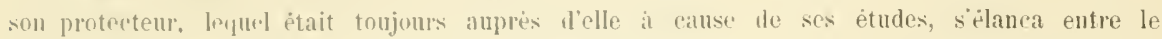
domestique et la femme. de sorte que le domestique lui fendit la tüte Quand elle rit ce qui itait arrive et comme elle se ligurait la douleur des parents, la femme senfuit de lit toute

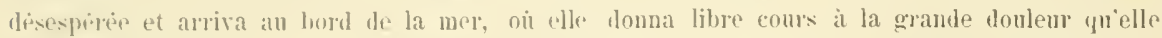
riprourait a cause de ce uni s'etait passes A ce moment. un mavire de pirates sapprocha, et les pirates sumparerent de la femme. Lorsue les pirates roulurent se remettre en ronte arec lenr proie, il surgit me forte tempete. qui menaca de faire sombrer le navire. Les marins.

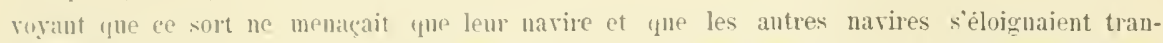
quillement et sams être infuiptés, tinrent conseil pour saroir quelle en était la cause et romment on pourait y remidier. Ms se déridiment à tiver au sort: celui sur qui tomberait le sort Serait jets à la mer. Le sort tomba sur la pieuse femme. On lui demanda de qnoi elle socenpait. et pournuoi Dien éfait si irrité contre elle. Elle raconta toutes ses a rentures, et ils eurent pitip d'elle et risolurent de la reronduire il terre Alors la tempete s'apaisa, et ils atteignirent sims eneombre la terre ferme, ou la femme descentit

le bon bien ent pitie de la pieusr fenme, de sorte fu'elle put se bâtir une cabane et quelle ent il sil disposition des arbres lruitiers at des arbustes quni fournissaient un remede contre toutes sortes de maladiss; entre autres. elle ponsait guérir la lépre, maladie qui se rneontre sonvent "n Orjent. Elle aequit ainsi la réputation d'être un habjle médecin, et il y

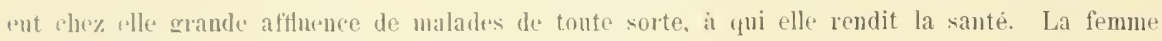
en devint riche et hemrelse.

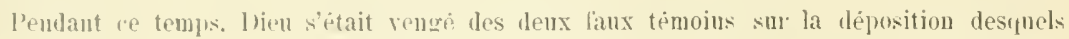

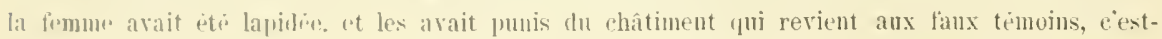
a-tlire la liprer. Le mari de la femme etait aussi rentre chez luj et réclama sa femme à sul lire. ('elui-ej ripondit yue sa femme, qu'il arait "rue frieuse, arait mene une vie

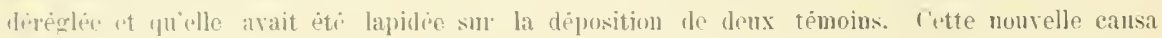
leamony de rolagrin et de somejs an mari.

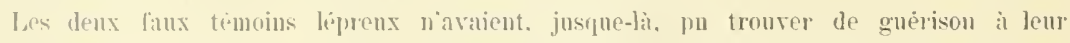
malarlie. lansurils entendirent parler de la liunense femme au bord de la mer, laquelle pourait guerir tontes les malalies, ils ressument de s'y rendre. Le malheurenx mari, pue la douleur

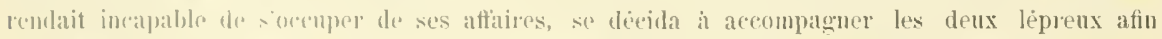
do hacher do la distraction a sa douleur. Tous les trois. ils arrivinent aupres de la femme, "t elle les resommt immerliatement; mais elle fit comme si elle ne les comaissait pas et leur

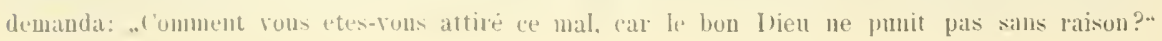


Alors les denx léprenx répondirent qu’ils araient commis quelques petits pẻchẻs. Mais la femme ne se contenta pas de cette réponse. Lille dit que lien était miscricordieux et n'enroyait pas une si grave maladie ponr quelques petits péchés; elle ne pourrait pas les aider, s'ils ne disaient la vérité. Alors ils aronèrent l'aftrense vẻrité concernant le fiux témoiquage et la lapidation de la lemme. Alors la femme dit ì hante roix qu'elle était précisément rette femme et "tue l)ien l'avait saurée. Et elle ajouta: ,l'nisque rons arez commis un tol péché et que rous avez tẻmoigné ainsi contre une personne pieuse t imnocente, vons avez élé punis selon l'Écriture, et jo: no penx pas rous gnérir, la rolonté de Dien étant telle." Lorsque lo mari entendit ces paroles, il lui avona tont de suite qu'il n'avait jamais donté de son imocrure. Ensuite ils restèront ensemble et vécurent henreux et bénis.

Morale, selon la parole du roi llavid daus le Psamme 34: "Quel ast l'homme qui premne platisir à rivre? Gu'il garde sa langne du mal et ses lères de proférer lit tromporie!a

\section{B}

Version d'Al Furay Batda Alsidda, traduetion.

(Ms. Londres, Brit. Mus. Or. 237, fol. 117 b)

Histoire de l'Arabe, de sa femme Ouriya et de son frère

On raconte qu'il y avait antrefois en Arabie un homme très pienx, doné de qualités aimables, qui passalit son temps en prieres et en jennes at qui se consicrait an service du TontPuissant. Or, il arait une fille nommée Onriya, qui etait li plus grande beantè de son époune; tont le monde vantait sa bonté, sa chastete et sa pietri, all elle était tonjoms ocupée à faire ses divotions et passait nnit et jour en pripres; s"etant detabée du monde, elle s'attachait à Dieu. Son père ètait m homme paure, et, bien qu'elle ent beancoup de prètendants, dont quelques-mus étaient d'un rang élevé, il ne ronlait pas domner sou consentement. A la fin, son père mourut, et elle resta plongíe daus nu profond chagrin. Après l'enterrement et les cérẻmonies funebres, Onriya se sépara du monde, s'adounant a la dévotion et à un commerce spiritnel avec bien. Sa prière constante était:

"() Sontien des abandomés, je snis vemue vers Toi et j'implore Ta grâce et Ta miséricorde; sois mon protecteur et méserve-moi de la malice des impies!“

An bout de quelque temps, m Arabe se presenta pour demander sa main. Ses amis, silchaut qu'elle était seule et saus protecteur, lui conseillèrent de l'arrepter, pnisçno c'était un parti convenable et un homme hompte et intigre; elle donna son consentement, et pen apress elle fut saintement mariée par les anis de son pere. An bont de quelques amnées, le mari dOuriya fut obligé de partir pour l'ígyptr dans me affine importante; mais avant son départ il pliça Onriya sous la garde de son propre frèe à lui, le priant de prendre hien soin d'elle et de pourvoir à tous ses besoins pendant son absence. Quand l'Arabe fut parti ponr l'kigypte, is frele vint, par conséquent, sonvent roir onriya it des henres fixes ot lui fommit re dont elle 
avait hesoin. Ün jour, cependant, le hasard vonlut que le frire saperçnt des charmes ot de la

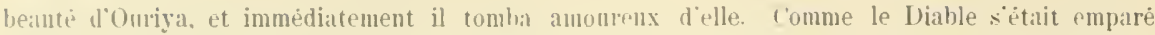
de lui, son anour pour elle ne connut plus de bornes: lis paix et la tranquillité labandonnèrent. de sorte quil devint presinue fou, et il commenga à chanter:

- laimour que j'ai pour toi est trop fort, pour que mon cueur puisie le combattre; la

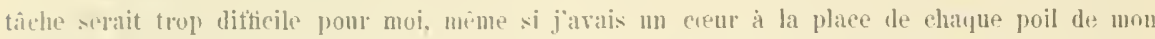
corjs: jottire ma vie contre un seul de tes chevenx; ce serait encore nue boune aftaire, si lon oftrait davantage."

A la fin, l'Amour lui tourua la tête; il n'eut plus de repos et ne put ni manger ui lormir. la peur et la honte le forcerent bien a eacher ses sentiments at a dissimuler ses tourments ef mortificatiuns, mais son pâle risage - le miroir de son cour - le trahit. selon le dire: „Combien mon curur sonthe à trarers mon visage! Pour sur, le risage recoit du cueur sa rlarti."

Ainsi se passa qunlyue temps. I'n jour: le frère se rendit à la maison douriya et $y$ resta plus longtemps que de contume, meme après unil ent terminé son trarail ordinaire; il commenca à proférer des paroles déraisomubles et à sompirer profondément, ce pui étonua heanconp, Onriya flle tronra assez singulier qu il restât dans sa maison plus longtemps que de

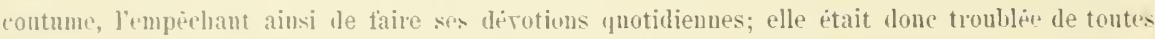
snrtis de pressutiments. A la fin, elle s'alressa à lui dans les termes suivants:

„.Frine, ne trouvez-rous pas une vous ittes reste trop longtemps iei aujouddhui? Te

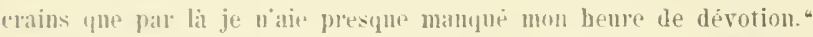

A prine ent-il ententu ces mots, une le fen de sa passion jeta des flammes, et il éloigna le. roile yui eachait son seret, en disant:

"La crainte du déshonuenr fera trainer trop à la longue cette attaire qui me concerne;

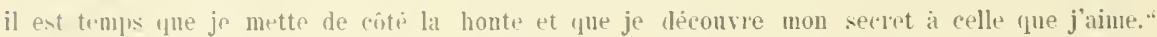

Alors il sadressal a elle 'il ces termes.

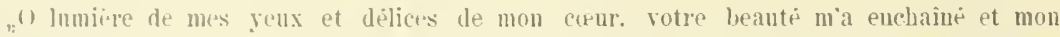
amour pour vons u'a conduit aux abords de li folie; jai perdn tont contrôle de ma passion, et je snis it hout de patience; ayez pitie de moi et acceptez-moi comme rotre esclave; de cum fut d'âme je serai à votre disjosition."

En cntendant cela, omiya fint saisice r?udignation, et le monde apparnt triste à ses

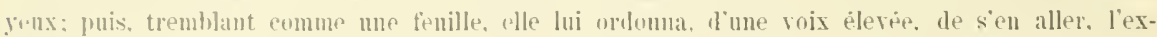

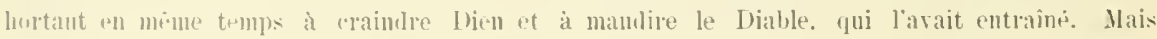
l'hume ne se soucia pas de ses paroles, et dit:

.. oljot nnique de ma vie, mon amonr pour rous dejpasse tonte description; rotre bean

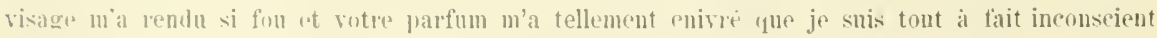
de tont tee qui txiste dans les denx mondes. Ni fremes ni amis ne me conseillent; car le contrible de moi-mime $m$ a quitte, comme la fleche quitte l'are."

Unriya lui dit alon's: .. Homme. abandome\% ces vains et inutiles désirs; craignez le Toutl'uissant et oreupez-rous de priéres; en homme honnête, garde\% le dépot sacré do votre frère

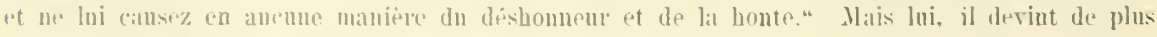

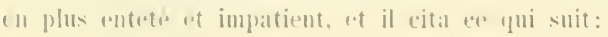


„O homme sage, à "quoi me sert maintenant rotre conseil? ('al l'Amour m'a dlja fraphr pour ma perte. Maint homme qui avait antretois porté un nom honoré a aequis de la motoriété daus ce monde par l'amour; il a perdu sa répntation et recueilli de la honte et sonffert la prerte de son curnr pour gagner du déshonnenr en amour."

Ensuite il plaida sa canse, en disant un’il avait èté décide fu’il tomberait amoureux d'elle et qu'il serait incapable de renoncer à elle. Il insista sur ce point, disant cin'il lui etait impossible d'abandomer l'objet qu'il désirait, sumtout après lui aroir fait comaitue son désil, ot "qne le seul remede $y$ serait qu'elle consentit a sa proposition. Mais Ouriya resta lerme comme un roe et fit lis sourde oreille à toutes ses persuasions et menaces; elle prélërit mourí aree homseur sque de vivre avec déshonnems.

Recevant de la part l'Ouriya refus sur refus, l'homme devint furieux et chereha a se venger d'elle. Done, mu jour vers l'aube, le frère de l'Auabe, aceompagué de ciug fripons impies, (fu'il avait subornés d'avance, pom yu'ils portassent faux témoignage sur la ehasteti dOuriya, s'introdnisit dans l'ẻtage supérien de sia maison, pendant qu'elle était absorbée dank ses prières; l'un d'enx entra d'abord dans le sanctnaire, et les antres le suivirent et le saisirent dans la chambre de dérotion. Le bean-frèe dOuriya s'empara ensuite de l'homme et dit:

„O fripon, pournoi êtes-vous iei dans la maison de mon frère? Je détacherai rotre tête de votre corps."

L'homme implora sa pitié et dit que ce n'étitit pas sa fante; e’était sur l'invitation de cette femme impie fu'il renait la voir charne mit. Alos les quatre hommes sonleverent le ropnin et le jeterent rontre Ouriya. Le tapage et les eris, 'ntendus à cette henre du matin, firent sensation dans le voisinage, et de tous les quartiers des hommes at des femmes se precipitèrent en désordre dans la maison. Onrịa etait comme frappée de la fondre à cause de re "fui se passait derant ses yeux, et, comme elle ignorait la trahison de son bean-frère, elle ne pouvait se rendre compte de re sui était arrivé cipendant, elle tomrua ses pensées vers celui (pui connait tous les secrets et, se remettant a sou hou plaisis, elle L'implora, demandant protrection et justice A ce moment, son bealu-frère commença à la rondoyer devant tous les prèseuts; il dit que, femme adultère, elle rninait l'homene et la maison de son frère et cu'il ne scrait pas un hommr, s'il u'obtrnait fu'on la jetât au fen, le lendemain, sur la plare du marché. Elle ne le regarda pas ni n'essaya de lni répondre; elle ne fit que pleurer amèrement et pria bien de la secourio dans sa detresse. Alors le counin de bean-frère la ponssa dans la maison, ferma hien la porte ot s'en alla La panve femme passa tonte la mit en priéres, attendant son solt. Le monstre dhomme se leva de bome henre le lendemain et alla droit anx maisons du ('adi rt des jnges. Le Cadi, après avoir appris tous les détails de l'atfarre, douna l'ordre d'amener Ouriya dexant le trilumal. Les sergents de ville allerent à sa maison, l'enlevèrent rudement et la conduisirent à travers les rnes, qui étaient pleines d'me populace insultante. Ouriya marchait les yeux baissés. (Quand le tribunal fut réuni pour entendre laffaire, le frère dit: "Cette femme est la femme de mon frère; je l'ai surprise an moment on elle se dishomorait avee un étranger, qui s'est échappé; elle mérite done toute la punition de la loi." Le Cadi pria alors Ouriya de plaider sa cause; mais, comme elle était trop désolée pour pouroir se defendre, elle ne fit que pleurer, et puis elle prononça ces mots: „Dien, le Tout-Puissant, est mon Juge: Tu es le senl „ui comnaisses les secrets des abandonnés et la vraie sitnation de cenx 


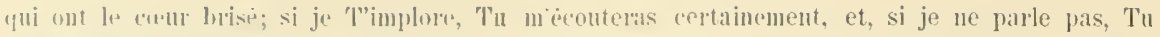
commitis le langage des muets."

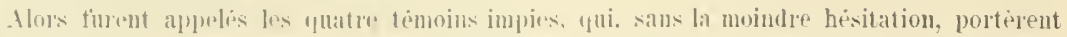

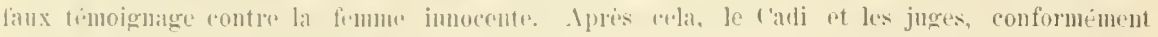

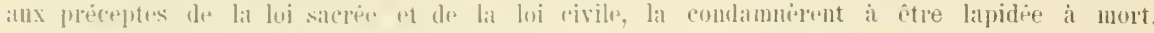

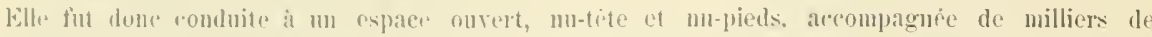

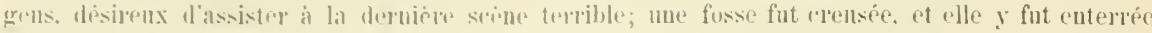

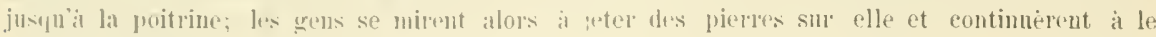

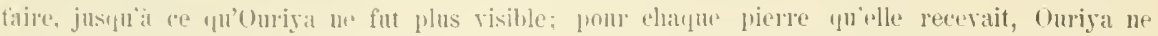

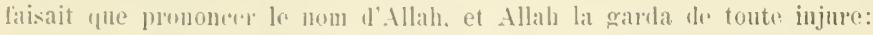

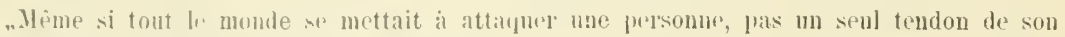
rorps ne serait endommigis, si bien ne le desialit."

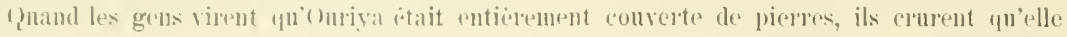
pitait morte, et ils sten allerent, deplorant la fin triste ret ignominiense de sa longue carriere piense.

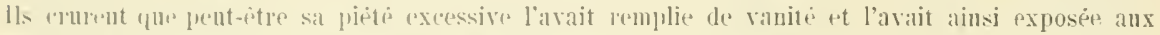
teutations du Iliahle; jour sur, les vojes de I lien sont mystérienses:

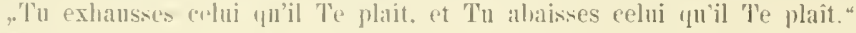

Duriya etait couchere sous le moureau de pierres, sanglante th sans commaissance. mais viraute; ear Dien arait decide yn'elle virait eneore; pendant re temps les gens faisaient des rethexions sur sa conduite: ynelyues-nus avaient sincerement pitie d'elle, tandis que d'antres appelaicut brutalement sa piriti un manteau de fraude piense.

A la tombere de la muit. eertain voleme de grand chemin passa, pau hasard, pres de la an rentant chez lui, apres avoir pilli une caravane en route pour l'Égrpte. son attention fut attiren par les gemissements de la malheurense femme, et il ordoma a ses hommes d’éloigner

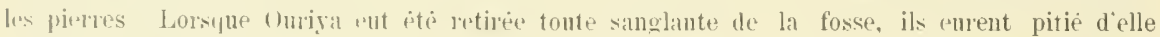

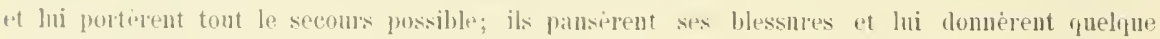

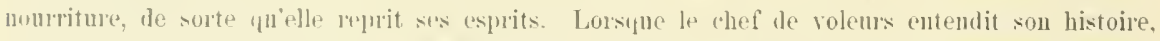
il en fut emu et lui temoigna toms les igands possibles; il se disait gne tout ce qu il pourait

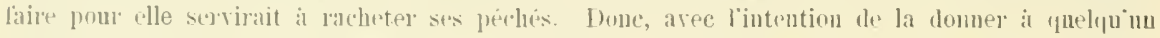

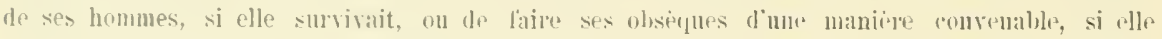
succombait, il la prit arec lui a la maison. Lorsinu la lemue do voleme, yui etait mue femme piensa et qui avait tonjours disapproure la rondnite de son mari, vit an sa rompagnie une fimme itrangipe. elle fut dabold surprise, mais bientit elle lut satisfaite, Jorsque la chose lui

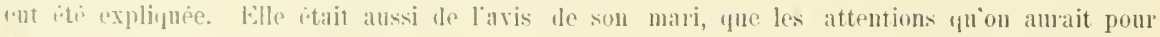

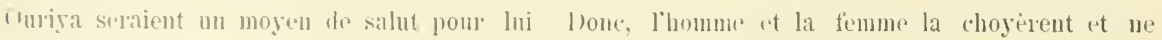

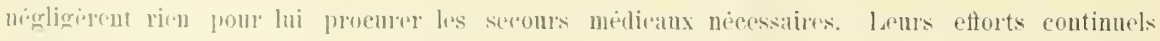

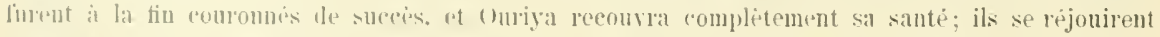

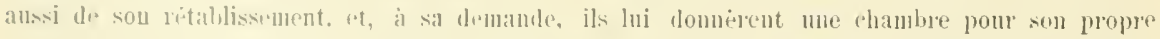

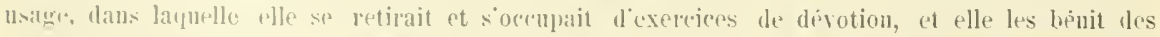
survicer quils hi araient rendus. En pen de temps, elle fut renomme dans la tribu pour ses vertus ct sa piité. 
Le chef de volen's a vait un esclave nègre, dont était le devoir de contuire les animaux domestigres aux champs et d'apporter de la forêt des lagots pour la consommation a la

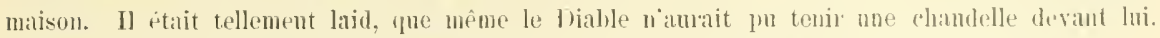
Un jonr, ref esclave entrevit la beante domiga et tomba immediatement anomenx dedle:

"Lorsque le mallene atteint $m$ homme, il fait tout ce yni est drisarantigenx."

Lat passion de l'esclave acerut channe jour, et à la fin il lui fut impossible de la contenir; et Ontya ignorait completement la rhose. I'n jour. le negre, la tromvint seule, lni revila son secret et la supplia d'avoir pitié le lui, et il dit:

"Qne je vous aime et que je rous aime encore, je le leclate a hante roix; et pour sirr les amourenx ne méritent pas d'être peudus an gibet."

Entemlant cela, Ouriya se mit à trembler d’indignation; elle le reponisa et menaca de faire romaitre sa conduite impndente a son matre et a sa femme, šil ne renongait pas, a lavenir, al ses intentions vaines et michantes. The expérience des plus horribles dans le passé at la crainte de noureanx et teribles tronhles dans l'arenir pponvanterent Ouriya. et, le curnr plein l'émotion et les yenx pleins de larmes, elle supplia llien, le l'nissant et l'Anguste, de la dèlivrer et de la protéger. Cependant, pour ériter les eritiques des méchantes langues, elle garda le secret sur cette aftaire. Une fois encore, pendant la mit, l'esclave nègre entra dins sa chambre de dérotion et chercha, avec véhémence et éloquence, a lui faire romprendre quil il etait éperdument amomeux d'elle et yn il lui était impossible d’abandonner la roie dans laquelle il s'etait engagé. Mais la bone Omiya resta inébranlable, préférant la mort an dishomeur: clle mandit le jom amquel et l'étoile sous laquelle elle etait née, disant:

"Celui dont l'étoile est néfaste ne prospèrera jamais, et le malhem est la plus terrible chose du monde; les troubles, les ipreuves et les privations atteiguent le gemre humin par lintluence des manvaises etoiles Mais, si celni qui est né sons mo bonne etoile renait à ramasser me épine pirmante, if la trouverait changèe, dans sa main, en me rose déliciense."

(1), l'eselare, se trouvant batm a thanne point et déch de tonte laçon. devint it lit fin

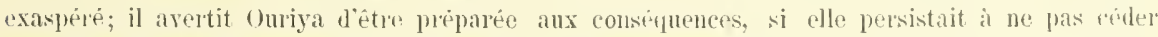
à son désir; mais elle se fia à la volonté ct à lat protertion de llien. Alors, le négre, craigmant les conséquences de son impudence of de son indiscrétion, si elle les faisait connaitre, chercha un moyen de se venger, et il forma mu projet de se debarasser d'elle, avant funclle füt capable de lui muire. Done, un jonr il se leva an milisu de la muit, süntroduisit dans la chinulue des enfants, on le petit infant de son maitre dormait dans son bereeau, et lui couja la gorge; ensuite il entra dans la chambre domiya et, après avoir aspergis avee le sang cuelques-uns de

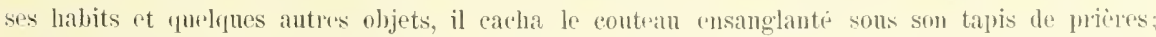
l’unocente lemme était profondément endornie et ignorait complètement que lo Jestin arait deride en alle aurait une nouvelle epreuve terrible a subir. Le lendemain an matin, les parent:

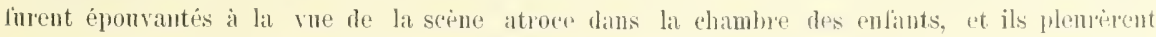
amerement la perte de leur cher enlant assassiné. Entendant les lamentations of les cris déchirants, les voisins arvirent en fonle, et ils furent remplis d'lorrenr a la vne de la terrible tragedir. Au milien de cette agitation, leselave nègre, ce diable incarné, se mit a crier a haute roix pt a courir çì et lá comme un fou, menaçant de vengeance lassassin de l'enfant, car il avait anué celui-ci plus que rien an monde, même plus que sa propre vie. 11 jura de ne pas se reproser, aviunt 


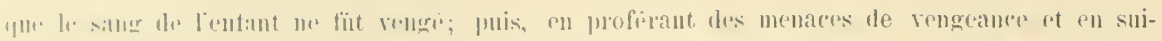

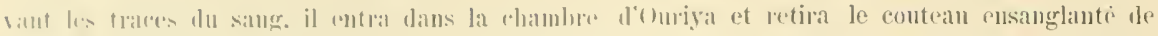

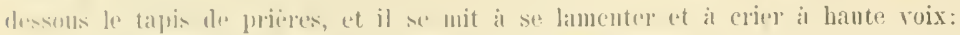

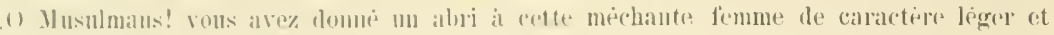

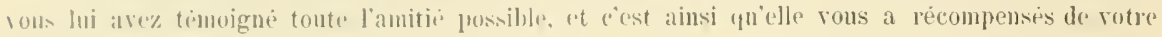

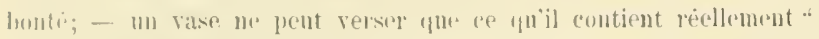

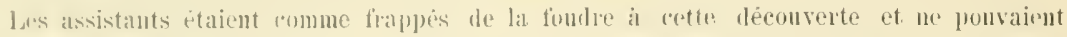

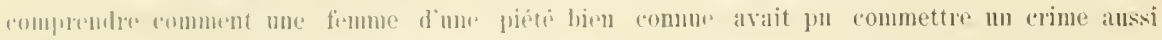

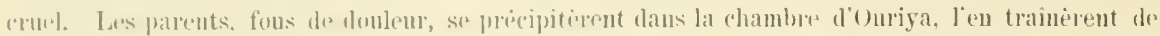

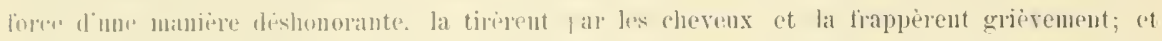

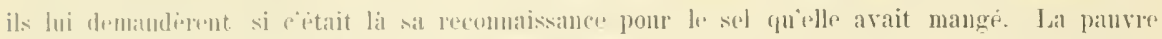

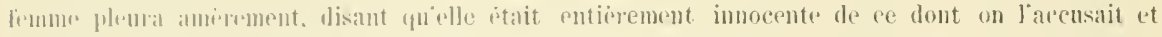

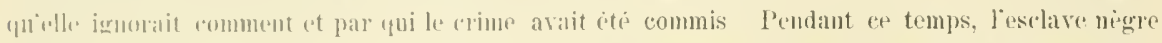

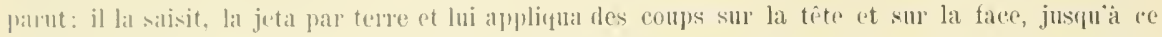

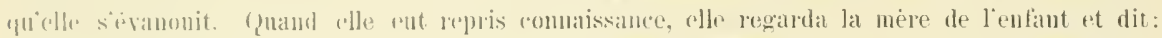

...'on l'anom de l)ien, ayez pitie de moi; nsez de votre raison et faites des recherches minutir llsi's sur cette aftaire."

Alors lexelare sortit mu grand contean et demanda a son! maitre la premission de lat

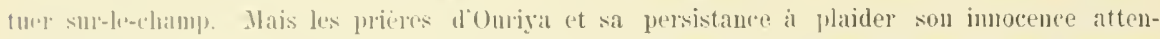

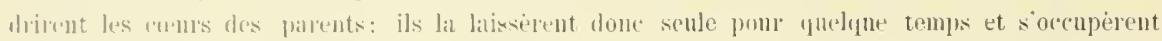
de linterrement du corps de lenfant assassiné. Ensuite le negre alla voir en particulier lo

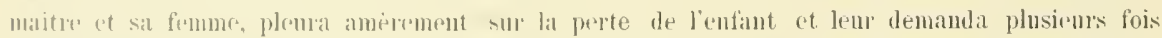

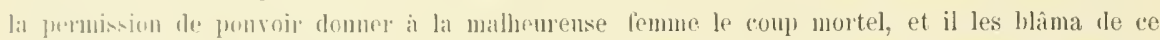

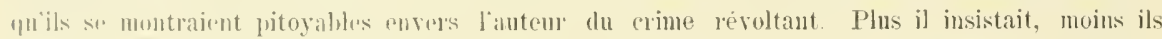
italient enclins in cédel à sa cruclle demande; car sa serérité daus cette affaire soulerait, dans linjurt des parents, des souncons sur de desinteressement de l'esclave. Le père de l'enfint,

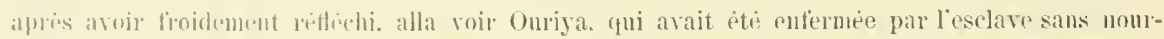
riture ni exall, avere lo corgs torture par les hlessures et par l'angoisse. Il eloigna l'esclave de

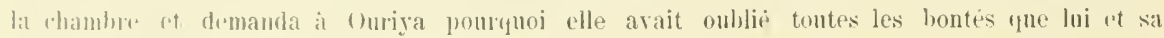
limume lui avaint teimoignies Omigya continta a plaider son innocence et le pria dattendre,

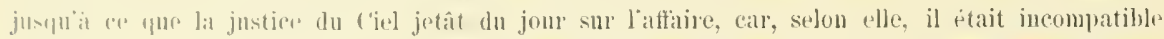

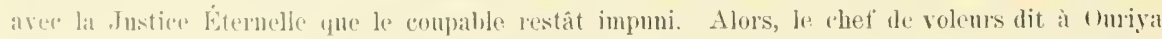

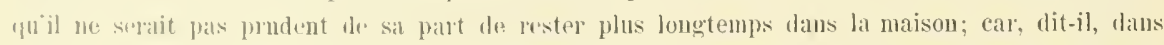
mu moment de crande indignation of de grand chagrin, il pourrait, lui on bien sa femme, perdre

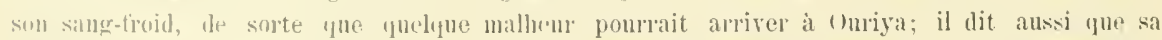

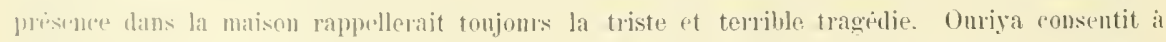
s'in aller, et. comme olle su preparait pour le depart, le chef de voleurs lui offrit cent dinars

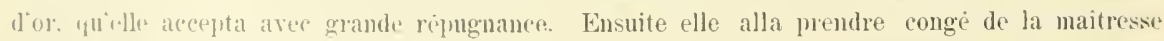
A. lil maisull, ot rel]e-ci dit:

„i rous avez fait dn lien. rous recerrez du licn en échange; et, si vous avez fuit dn mal, le mal retombera sur vous; - ta robe sera aspergée du sang de ton cour, si la juste punition du Destin tateint." 
Entendant cela, Ouriya plenra ef, haisaut la main de sa bienfaitrice, elle la cuitta.

Après avoir tquitté la maison, ()uriya se retira, pour or jour, dats un endroit solitaire. afin d'éviter la vigilance et les persécutions de l'esclave nègre. Le Imdemain an matin, elle partit de lá, sans bien savoir oi clle allait, et, après avoir traversé mu vaste lésert, elle arrival à une ville. Là, elle rencontra une vieille femme. à qui elte demanda un abri, disant qu'elle était une etrangèe et une femme lomete; la demande donriya fut accorles? ot, moyennant de l'argent, elle fut pourve de nourriture et d'antres choses indispensables. Apres s'être reposie denx on trois jours, Ouriya pria la vieille femme de laceompagner an bain; on ronte, elles entendirent sondain quelque tapage, et, regardant antour d'elles, elles virent un jenne homme, les mains liées et une corde antour du con, qui, accompagné d'une foule bruyante, était brutallement pousse en avant par la police. Oniga demanda a la femme la canse de cet incident, et la femme lui racouta que le jenne homme arait détomrné l'argent dı roi et 'pu'on exigeait quil le restituat. Ayant appris par la vieille femme que la somme dlitommée montait à cent dimars d’or. Ouriya la paya et olitint par là la mise en liberté du jenne homme; par cet acte de générosité elle éveilla radmiration de tout le monde. Le lendemain, J'homme vint vois Gnriya, la remercia virement de sa thès grande bonté et lui dit:

"Jusinuà la fin de ma vie je roublierai pas le grand selvier que vous miare\% rendu, et je resterai pour tonjours votre obéissant serviteur."

Ouriya répondit que tout ce pn'elle arait fait avait èté pour plaine an Tout-Puissant et non pas pour obtenir une récompense terrestre, et elle ajonta:

"Que Dien vous bénisse pour la peine une vous vous êtes domée en venant chez moi; mais à present il vous faut vous en aller."

Pendant ce temps, J'homme avait entrevo sa heauté, et il tomba immediatement amonrenx d'elle; et, malgré ses protestations, il la suivit partont, sadressant à elle tout le temps en termes affectionnes et ayant pour ello des attentions spéciales A la fin, lorsuluils liment arrivés à un désert, le jemne homme, tronvant Ouriya seule et personne a portéc de la vue, dérlara ouvertement son anour pomr elle en ces termes:

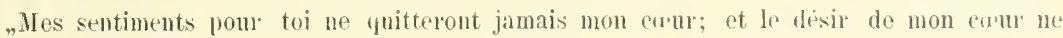
sera pas comblé sans toi; et mes mains ne se retireront jamais de ta robe, méme si ma teite est separie de mon corps, perdant que je te poursuirrai."

Entendant ces mots, Omiya liut remplie d'indignation et le reponssa rudement, et, comme Jhomme essayait de la violenter, elle lui demanda si cette manière de la traiter atait la réconpense du service qu'elle lui avait rendu en samvant sa vie, et elle lui diclara que sa manvise intention resterait complètement vaine:

"Faire du bien an méchant e'est comme si l'on faisait du mal au bon."

En pronongant rette maxime, (Inriya s'èloigna de l'homme, et ils continuirent leur royage. A la fin, ils arrivèrent anx bords diune rivière, où. à ee moment, une caravane se reposait L'honme s'avança et, s'adressant an chef des marchands, entra ell conversation avec lui. Pendant cette courersation, il parla en termes ardents de la beaute d'()miya, qüil disait etre son esclave, et en même temps il exprima son desir de la ventre, parce quielle avait èté assez rebelle à ses ordres. Alors mu des hommes alla jeter un coup d'eil sur ouriya, at, il son

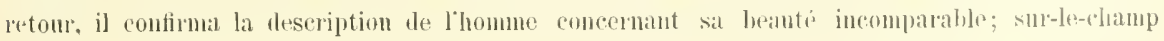


mu marlue fut ronclu wh vertu dupuel la paure fimme abandonnée, qui ignorait entierement ce

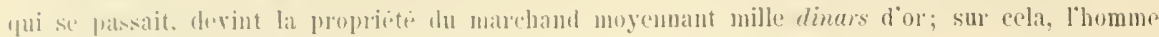

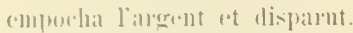

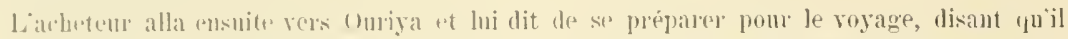

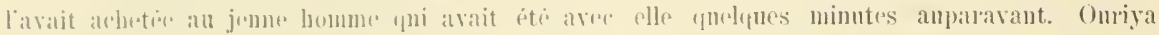

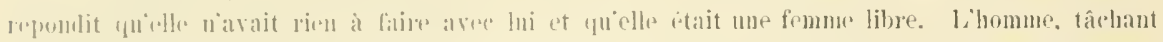

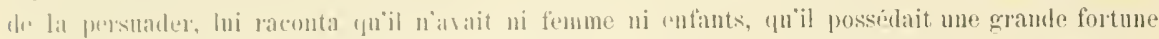

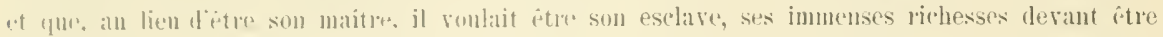

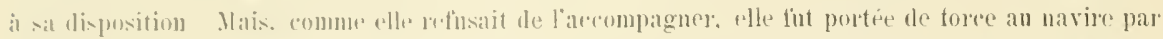

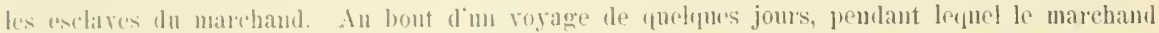

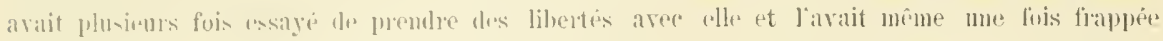

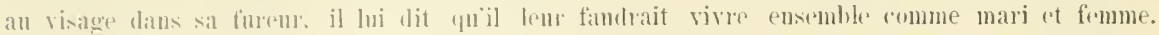

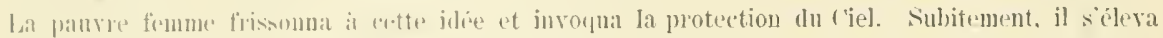
mur violente temprite, acompagnes de tremblements de terre, de tomnerre et d'exlairs, fui faillit

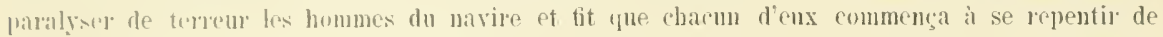

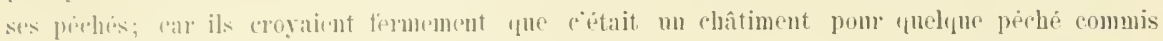

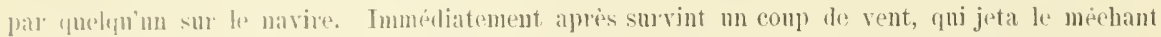

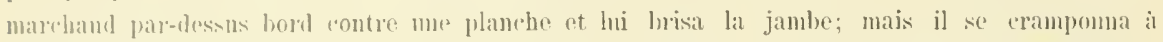

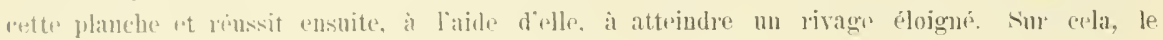
navire rontinua sa ronte et allorda estin un port. appelé sahel.

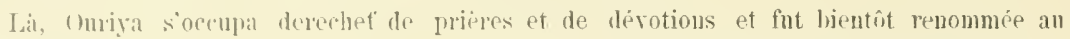
foin pun ses vertus of sa piete. Lorsene lat reputation domiya arriva justuan oreilles do roj, il but saja d"un vif disin de la voir; done. un jour, acrompagné d'une nombrense suitu. il

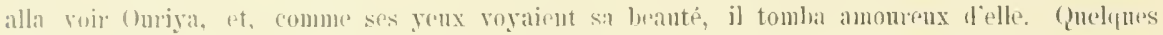

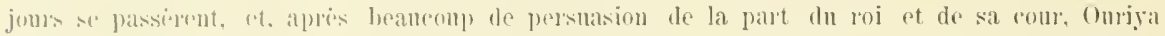

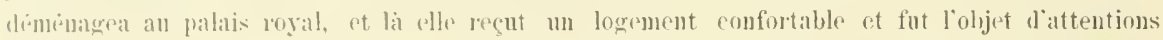
spéciales. rependant, la passion dn roi devint de plus en plus forte. de sorte quelle dépassil

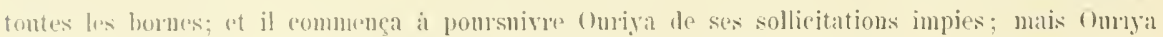
ne unitta pas la bome ronte et trompa chanme fois he joi dans ses projets pervers. Enfin mu

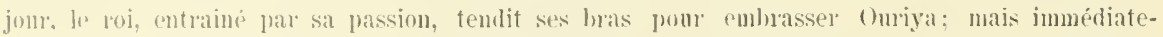
ment, anme Ouriya juphorat la protection du ("iel. Lien frappa de paralysie l"un des bras du

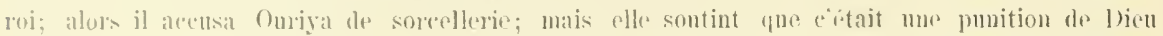
it ranse de sa manxalise action et lui conseilla deses repentir. Après quil se fut repenti et qu(mriya ent invouné Dien pour son yötablissument bien guérit le bras paralysé dn roj

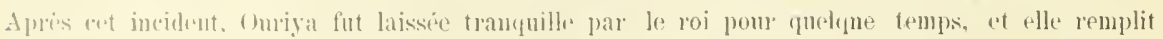

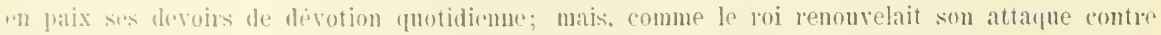

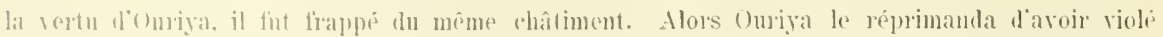

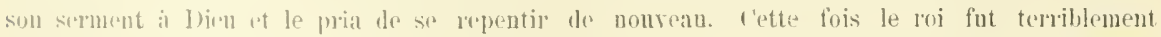

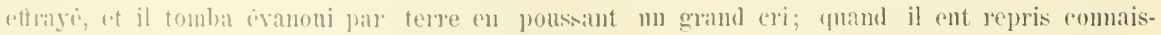

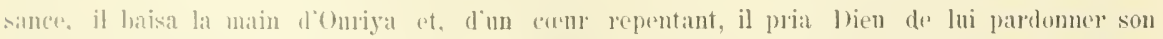

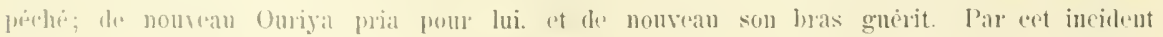

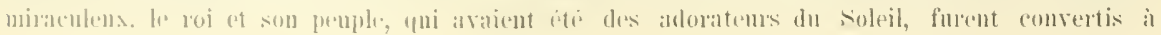


l'islamisme, et cet événement fit un tol brnit ru’a partir de ce jour cenx qui étaient malhenreux renaient même de contrées éloignées chez Ouriya, afin d'être délisres, par ses prières, de lenrs maux.

Dans le cours des années le roi mourut, et, comme il n'avait fus laissé d'enfant, les gens du pays. grands et petits, choisirent manimement ourya pour leur souveraine. De homes lois et contumes furent alors établies par elle, et la paix et la prospririte entrerent dans le pays; le penple conmença à vive daus un contentement parfait ot à prier constamment pour son salnt, désirant an'elle ent une longue rie. Ponr loger confortablement les royagenrs, Omriya fit construire queliues anberges ou ils pussent être exmpts de toute dépense, et telle était la générosité de son caractère qu'elle les pourvoyait même d'argent et d'autres choses nécessaires. „quand ils s'en allaient. et qu'elle donnait des habits royanx et d'autres cadeanx mrécieux à ceux qui s'en montraient lignes.

Quand l'Arabe, mari d'ouriya, revint d'Égypte et tronva sa femme partic et son frère arengle, il fut terriblement bouleversé. A ses anestions, son lire lui raconta, en mentant, qu'Onriga avait mené me mavaise vie; quelle avait été smrunise an moment même ờ elle se rendait conpable d'intidélitė; qu’elle avait été conduite devant le triłnual du Cadi; que, malgré tous ses efforts pour la sanver, elle avait été condamnée il etre lapilée à mort; et enfin, que rette malhemeuse affaire avait lait sensation an loin ot cu’il ptait lui-même devem avengle a force de plemrer sur le malheur de son frère. lidrabe, entendant rette histoire, plenra amèrement at exprima sa smprise de ce yu'une femme de sa piété et de son intégrité avait pu commettre cet abominahle crime. ('enendant, l'Arabe ne put oublier Ourya; il plemait sonvent en pensant à elle et disait sonvent re sui snit:

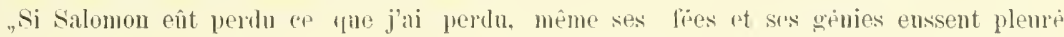
par pitiè pour Salomon."

Après rela, toutes les fois que le lière rntendait les lamentations de l'Arabe sur l'intillèlité drmiga et sur sa porte, il faisait des remontrances it l'Arabe et tâchait de le consoler, disant qu'il derrait être reconnaissant d'avoir perdu me telle fomme et qưil devait épouser une antre lemme. Nais l'Arabe naimait pas ce que disait son frire et le disappronvait; cependant il ne disait rien.

Pendant ce temps, le bruit s'était répandn partont une certaine femme arabe régnait sur le penple de Sahel et qu'elle gouvernait son pays avec honté, liberalite et justice; on disait smtont cupelle petait l'incarnation de toutes los vertus féminines et de tontes les pualites saintes. ret que par son interesssion anprès de Iren cenx yni étairnt malades avaient été délivrés de lems manx.

L'amom fratermel se confirma, et l'Arabe partit pour Salsel arec sou frère, espérant cque sa vue lui serait rendue par les prieres de la sainte reine. En route, ils sarrêterent ì l'endroit oin dememait le chef de volems, le mattre de l'esclave nègre qui avait si impitoyablement persécuté Ouriya. Le chef de rolenis, ayant appris le but un voyage de l'Arabe, résolut anssi de se rendre à Sahel, à cause de l'esclave nègre, qui avait été frappi de paralysir dans sus membres, et ils se mirent donc ansemble en ronte. An lout d'm royage de quelines jours, ils rejoiguirent me vieille femme aux bords l'me rivièe; en plemrant et 'n gémissant, plle condui-

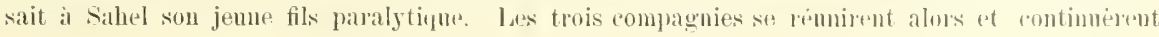


lem route re s'embarquerent bientêt sur un navire. Au cours de leur voyage, ils arriverent a une ile; lat, des cris et des lamentations parvinent it lems oreilles; ils penserent dahord que l'ile fitait hantie par des revenants et se disposaicnt à la quitter, mais, comme les eris et les Iammations contmuajent, jls changèrent d'aris ot so mirent a chercher ce qui prourait en être lit ranse. Ils dicourrirent $n$ homme presine réduit il l'état de squelitte par les soufliances et les privations, avee les yeux profondement enfoncés dans leurs orbites. Le pantre homme lemr raconta en quelgues mots antrecoupés qu'il arait ité une fois un riche marehand. mais que. comme il avait mahmenf ume frmme piense qu’il arait achetée à un jeune homme dans un desert. son navire arait été surpris par un tempête, pendant larnelle il ètait tombé pardessus borl, s"etait eassé la jambe et avait anlin ité poussé par les ragnes à cette ile, supporté par une planche. Ils le recueillirent done sur le navire et lui dounèrent il manger et à boire, juscu'à re ynüil ent regaguri ses foress. Ensuite ils lui racontẻrent le but de leur voyage, et, sur la demande de l'homme, ils lui permirent de les accompaguer à sahel pour elhereher guérison à sa jambe cassée.

A la lin ils arriverent à leur destination, of la nonvelle de leur arricée parvint aux aroilles de la reine. ('omme elle arait en une vision la nuit précédente, elle comprit tout de suite qui ils itaient, et elle ordonna done à ses hommes de les conduire à une des meillenres auberges et de les hien traiter duant trois jours, ronformement aux nsages des pays musulmans. Le quatrieme jour, elle ordonna qu'on les amenat devant clle, et elle lenr demanda qui ils étaient et pouryuoi ils remaint et quels étajent leurs max. Après avoir appris les détails de chaque cas, la reinu mandil qu'on tint une assemble publique (dabar), à layuelle tons les hants dignitaires du royaume furent conviés. Ic palais était décóé d'une manière splendide, et le trône, ‘ui était alors placé dans le salon l'andience de la reine, était richement orné. Quand tont fut prêt, la reine s'assit, rêtue de riches rêtements, la comronne sur la tête. Les nonreanx renus furent alors amenés devant elle; mais, lorsyu'ils virent la magnificence du palais, ils désespérèrent de pouvoir atteindre leur but is un endroit ou tant de splendeur mondaine était visible. Alors Onriya, afin de justifier son caractère devant le mari et pour mettre tons cenx qui s'y trouraient réunis au romant da vai itat des choses, leur enjognit à chacun de raconter fidelement son histoire "t les ivinements yuj avaient amené le malhemr de chacun, et en même temps elle leur fit sriverement sentir l'importance du fait „que, s'ils achaient quelque chose on disaient quelque rhose yui etait finx non semlement elle pouratit déenvir la rérite an moyen de cortain charme qu’olle possídait, mais quanssi le désir de leurs cururs ne serait pas comblé.

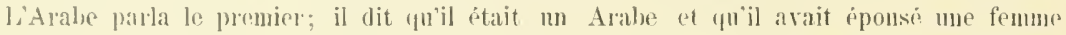

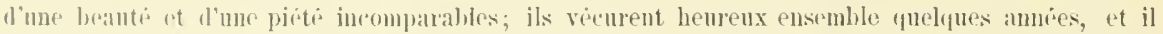
l'amait bancoup. Malhomrusement, il fut obligé de quirter sa maison et de se rendre en Hegpte pour atfaires, et il wargea son fripe dre prendre soin de sa femme en son alsence. A

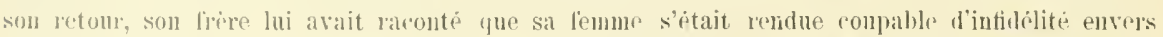

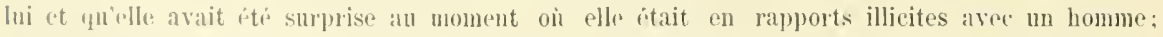
qu'clle avait pal conserfuent, conformément à la loi de l'Islam, itri condamuée à être lapidée à mort; que la douleur de son frire, cansée par la houte et la mort le la femme, avait été si

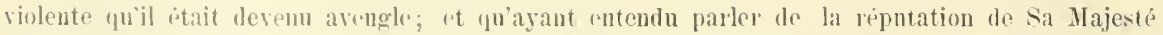
de pouvoir guerir toutes sortes de maladies par ses prieres, il avait éte pousse par son affection

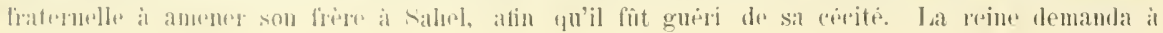


l'Arabe ponrquoi il était persuade de la verité de l'histoire concernant l'infidélité de sa femme. LiArale repondit quil ne pouvait comprendre comment me femme de sa vertn et de sa religiosité arait ju commettre nu tel pèchè, of que, faute d'mue solution satislaisante de ce mystére, il arait tont le temps sonffert des peines inoü̈es, tellement qu'il craignait de deveni a reugle lui aussi

Eusuite le chef de volenrs dit qu'a eanse de l'ètat praralytique de son eselave négre. qui arait été sa main droite et ‘ui smroillait tout son ménag̣e et ses antres aftaires, il avait complètement négligé son métier poul quelfue temps et "que cetait pour coli qu'il amenait l'eselave a sahel pour etre gnèri.

luis la vieille femme dit fu'elle avait. un senl fils au monde, qui avait été franzé de paralysie, et elle supplia la reine de bien vouloir prier pour sa guérison.

Eufin, le marchand raconta anssi son listoire.

La reine exprima la satisfaction qu'elle avait épronvée en écoutant lens récits et lem lit que, commo elle n'ètait pas médecin. la senle close qu'elle put faire, e’ètait de prier l)irn pour leur guérison; mais elle ajonta pue ses prières ne seraient cntendues qu'a coudition tue charque personme malade fit un compte rendu véridique et complet de son malhens, et que, si quelqu'm disait nu mensonge, il recerrait mu tel chatiment que ce serait nn arertissement pour ceux tui oseraient jamais mentir en la présence de Sa IIajesté. Quand les pauvres hommes cntendirent cela, ils furent lans un crnel embaras; ils pensaient qüils devaipnt préférer les maladies à la honte et an déshonnewr causé par la rérélation de la rérité. Afin de sortir, pour le noment, de cet embarsas, ils demanderent done dn repit pour me mit, re cui lenr fut volontiers acordé. C'ependant, ils passèrent tonte la nuit sans repos, parce qu'ils voyajent, à lenr tris grand chagrin, qu'il ne lenr restait d'antre mogen que de raconter la vérité entière.

Le lendemain an matin, la conr s'etait runie avec encore plus le pompe et de magnificence, et tous étaient présents. Le marehand s'avança le premier et, après avoir salué la reine, il raconta son histoire: comment, aux bords d'une riviere, il avait une fois achete a un etranger une jeune et belle femme; comment il l'arait fait transporter de force a son navire, avait commence a prendre des lihertes avee elle et l'avait enfin frappée an visage, paree qu'elle ne cédait pas a sa prière; comment la tempete s'éleva, le jeta par-dessus bord et lui cassia la jambe; enfin, comment il arriva à Sahel. La rine crut ce qu’il disait, se prosterna sur le sol et pria pour lui. le marehand gnérit immédiatement et completement, à la grande admiration des assistants.

Ensuite le jenue homme, conduit par sa vieille mère, s'avança ef raconta comment il avait détom'né l'argent d'nn roi et etait en ronte pour le gibet; comment nue femme arabe piense arait obtem son relâchement moyemant cent dinars d'or; comment il était plus tard tombe amourenx d'elle; comment il la vendit à un maschand anx bords d'nne rivière, et enfin, comment, la même nuit, il fnt frappé de paralysie. Omiya pria de nouvean, et l'homme reconvra anssitot sa sautè.

Ensnite l'esclave nègre fut appelé à raconter son histoire; mais, à canse de la présence de son maitre, il hésita d'abord beancomy à laire son récit et resta quelque temps silencienx. Ouriya dit que, grâce à la magie du charme, elle savait qne l'esclave avait commis un crinc affreux et que cètait là la raison de son silence; une, s'il ne racontait pas la vérité lui-mềıe, 
He on rivelerat la nature; et que, daus re ras, olle le sommettrait a une punition exemplate

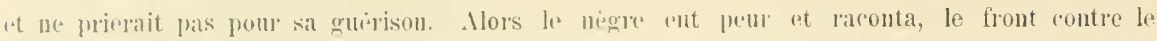
sol, comment son maitre. le thef dre roleurs, avait une fols domne l'almi à une femme très piruse, dont liselare ptait ensuite tombe incrdument amourenx; comment, apres aroir subj de

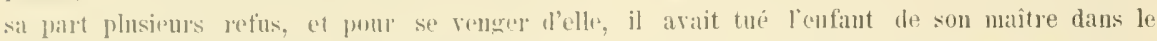
bureall, arait eaché le conteran ensanglanti sous le tapis de prières de la lemme junocente,

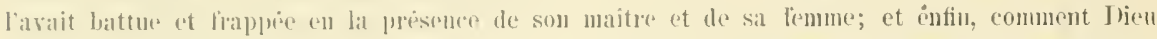

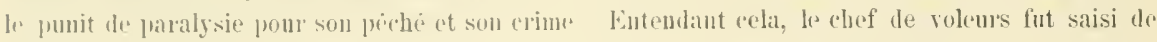
(hagrin et deftroi. et la tete lui tomra. Il supplia la rejue de ne pas prier du tout pour Festlare, mais de lui permettre de tnel immédiatencut le négre aree son ipée. La reine

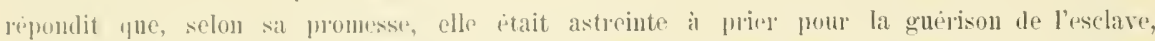
puisyu'il arait dit la verite. et qu'ensuite il dependrait du maitre de traiter son esclave comme il le vouhait. Ells pria done, et le miggre gruerit.

Eufin, l'homme avengle lint prie de labe son recit. Il dit d'alhord que son frère, l'A rahe, arait dijat raconté a la conr les cireonstances qui avaient amene son triste malhem; mais,

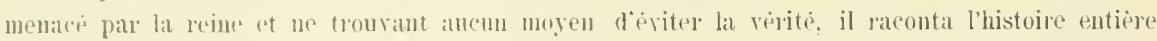

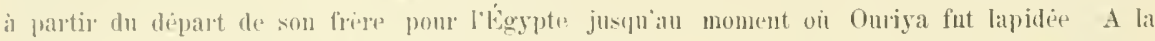
reviation des faits reck, l'Arabe poussa subitement un cri aigu et tomba sans connaissance

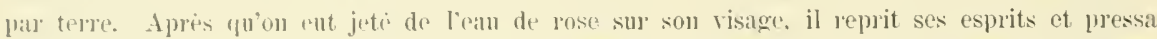
la reine d'ordonner inmediatement de laire liphider à mort son frère, ainsi que celui-ei arait

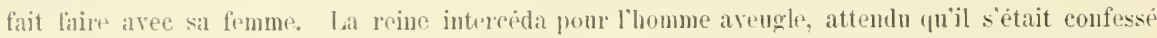
et s'ritrit repenti sincerement. Essuite elle pria pour lui, et il recumra la vue.

Lat reine demanda alors a l'Arabe comment il arait lintention de traiter son frèe

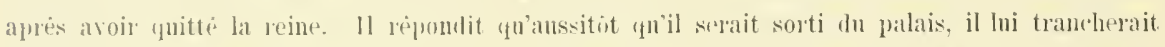
la tête; ot il confirma son intention par un serment. la jeine demanda a l'Arabe sil ronlait fraiment renger le sang de sa femme par la mort de son prople frere, quoicu'il sôt cu'on pourait bien trourer un sulstitnt pour une femme, mais ancun pour un frère. et elle lni ofìit anssi pour femme me des plus eharmantes des dames qui etacnt lit aupres d'elle. Mais l'Arahe, apres aroir refuse aver recomnaissance loffie de la reine, dit qu'il arait juré de n’èpouser ancone femme apriss la perte de sal sainte lemme cherie, ut, en frusant il alle, il fondit en lanmes. La reine demanda alors it litrabe quelle réeompense il lui domnerait, si, par son intercession ampres de Dien, elle rendait sa temme a la vie. Ayant vo les mimeles faits par la reine, l'Alabe déclara que, si la rejur ressuscitait sa femme, il lui resterait recommissant juscu’à la fiu de sa vie.

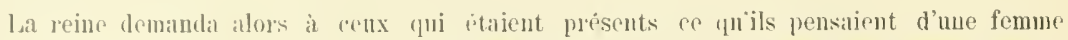
yni aurait subj tontes les tentations et ciprenves dierites far ces misejalules hommes et qui

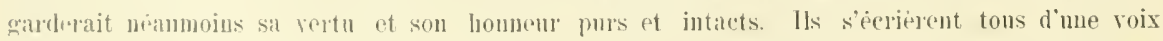

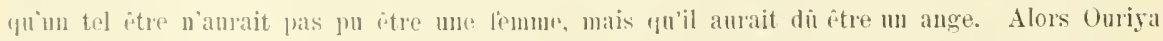
ita son roile et dit que chait elle yni arait subi tontes ces iprenves, et elle embrassa son

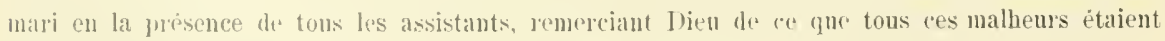
bumeusement termines. 
Ensuite Ouriya s'adressa a son beau-frère et lui demanda quelle faute plle avait hien pu commettre pour être si impitoyablement maltraitẻe par lui; l'homme ent tellement honte de. lni-même tu’il demenra interdit et ne put profërer une seule parole, même pour se délendre. Puis Onriya s'adressa au chef de volemrs, en disant qu'elle etait cette même f'emme malhempense et délaissée qu’il avait retirée de dessons le monceau de pierres, dont il avait pansé les blessures et qu'il avait abritee sous son toit; le ehef de voleurs s'exinsa humblement de l'avoir maltraitée le jour de la dẻcouverte du mentre de son petit enfunt. On'iga l'excusa de ce cui etait arrive dans des circonstances attennantes et hi amonça son intention de le récompenser. royalement de ses bons services. Ensnite elle s'adressa à l'esclave nègre et an fils de la vieille femme et leur demanda une explication de leur conduite iufâme enrers elle; ils étaient tous les denx excessivement hontenx et ne dirent rien; enfin, elle pria le marchand de présenter une justification de sa conduite; il ne fit que demander pardon. Sur cela, la reine etendit sa miséricorde et son pardon id tous et à chacun deux, et elle supplia son mari de pardomes à sou lière et le chef de volems de pardonner a son esclare. Apris cela, elle les traita d'une maniere rraiment royale, et, après les aroir chargés d'argent et de riches culcanx, selou les mérites de chacun, elle les envoya chacm dans son pays natal. Ils repandirent aiusi la bonne réputation d'Ouriya dans des contrées éloignèes.

Omiya et sou mari vécurent longtemps hemrenx ensemble et gouremerent leurs sujets avec une justice et une gémerosité sans ëgales. A la fin, elle se fatigua des grandeurs terrestres et commença à langur apres la solitnde pour pouroir en paix adorer Dien of eitre en communanté avec Lui. In jour elle proposa donc à son mari de régner senl; mais il refusa cette? propositıon et préféra se retirer avee elle des atfaires mondaines. Alors Uuriya abdiqua ell favem de sou bean-frère et se sépasa du monde pour être réunic á Dien. Durant tonte sa vie, Ouriya fint le modèle d'une lemme parfaite et piense; et, après sa mort, elle laissa derriere elle un noble exemple pour les antres. Bien qu'elle ne soit plus de ce monde passager, son nom virua pourtant a tout jamais.

\section{C}

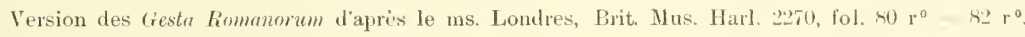

(Fol. $\left.80 \mathrm{r}^{\circ}\right)$ Menelans in cinitate Romana regnanit, yui filian regis Hungaris in vxorem aecepit, que erat pulcra et operibus misericordie plena. Imperator, quando semel in lecto sno iacuit, T'rram Sanctam risitare eogitabat. Die crastina vocanit ad se imperatricem et rnicum fratrem sum et ait imperatrici: "Karissima, a te celari non dehet, ynod memm propositum rst totaliter Terram Sanctam visitare; vade prineipaliter constitno te dominam toeins imperii mei ad ordinandum quod mihi et meo populo sit rtile." At illa: „Ex yno aliter non poterit esse, fiat volumtas restra. Ego vero cro sicut turtur in restra alsentia, qui socium sum amisit, ¿nia spero cum sanitate domm renietis." Imperator reso eam rerbis dulcibus confortabat. Osculum ei tradidit et ralefecit ommibus et at 'Terram sanctam perrexit. Frater vero cius 


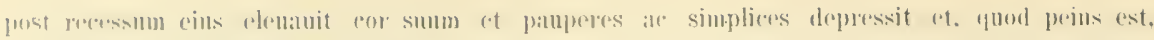

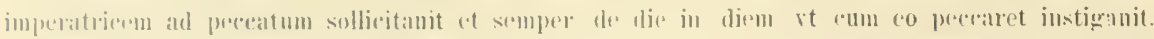

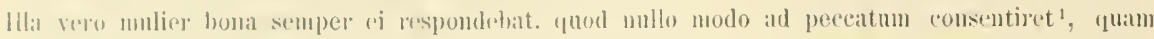
din dominns vius viurret. lile vejo hoc non olstante, quando eam solan inmeniebat ${ }^{2}$, per omuem

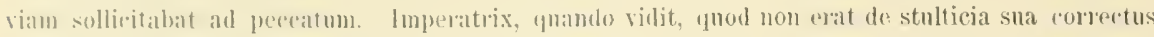
.t de mullo modo emendare vellet, vocaut tres nohiliores vel ynatuor imperji et ait ris: .. Karis-

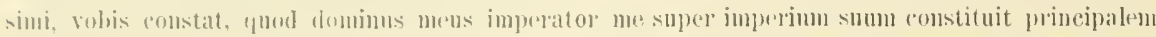

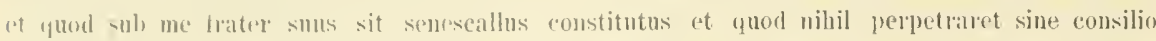

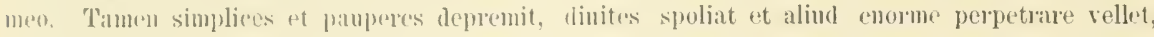
si posset. l'ropter que precipio vobis, vt lontiter ligatum in eareere ponatis." At illi: „Regina, mala inlinita post recessum impreratoris operatus est. Ideo precepto vestro obediemus." statim manus in rum injecerunt er vinculis ferreis ligauerunt et in eareer posuesunt, vlo per multos

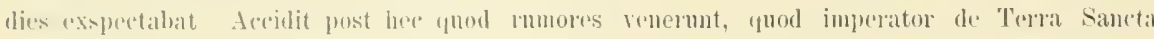

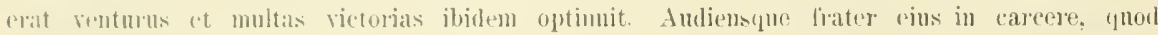

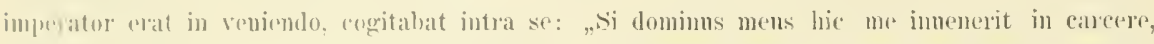

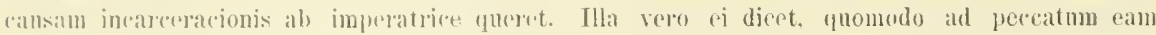

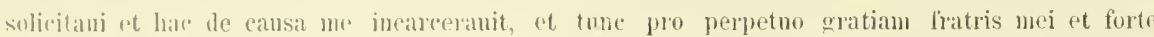

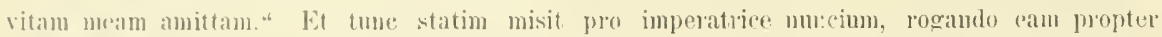

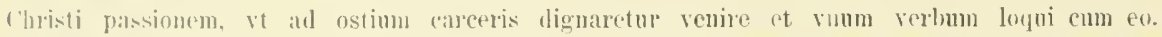

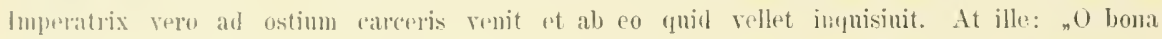
dumina. miserrenini mei! si frator mens hie me inmenerit, filiss mortis sum ego." ('ni imperatrix dixit: .. ii scirem, quod a stulticja tha dexisteres, et honestum ammodo imunirem, gratiam

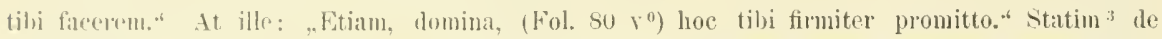
carere am ahduxit'; haheari ut tonderi freit emm. Hoc facto se vestimentis preciosis induit", et ei dixit ${ }^{6}$ : .Ian, boue friter, dextrarium ascende et reni mecum obuiam domino meo." At ille: "lrosto sum." Imperatrix vero perrexit obuiam domino suo cum eo cum multis aliis dominis. Et, sicut in via repuitabant, quidam ceruns ante ros cucurrit. Omnes rero equntantes in via illum videntes cum cormilons agili cursu cerum insequti sunt, ital tuod nullus inxta imperatricem remanit nisi frater imperatoris. Et cum ille boe ridisset, ait ei: "Womina, hie est vna forestil seretal. Din est, ynod tibi de amore loxpelas. Veni modo ot conscuti mihi, vt treum rooam." Ait imperatrix: "(vid est hoc? Hesterua die stulte perphtuan correctionem promisisti et de carcere te abduxi et ad buc tam cito ad staltiriam tuam vis residiuare ret relire. Iam dico sicut prius: nullus mecum tale perpetrabit nisi donims mens imperator, yni hoe de iure habet." At ille: „Nisi mili consenseris, te per erines super arborem in foresta suspendam, vbi mullus te inneniet, et sie mala morte morieris." At illa: "hicret caput memm anferns et omnia grenera tormentorum in me exerceas, munquan cousensum mernm ad tale peceatum habebis." Hlle her audiens denudanit illam excepta sola camisia ret eam per arines super qnerom smspendit et equum sum ad arborem liganit. Hoc facto ad socios suos equitanit demuncians eis, quod multitudo populi ab eo imperatricem rapnerat. Vnde factus est dolor magums per totum imperimm. T'ost tres dies cnm quidam comes per illam

$$
\text { Ms. cosentiret. - }{ }^{2} \text { Ms. tueniet - Ms. Sat. +Ms. adduxit. - }{ }^{5} \text { Ms. se induit. - }{ }^{0} \text { Ms. duxit. }
$$


forestam renatus esset et post vulpes ante canes cucurrit, eanes inserpunti sunt, in currendo odorem domine senserunt et sum cursum dimiserunt et versus arborem agili cursu gressus: suos direxerunt. Comes loc videns admirabatur, percussit equmu cum calearibus et inserutus est, donee ad arhorem renit, iu thi domina suspensa erat. ('omes rero, cun pam ridisset suspensan per crines, admirabatur eo (quod pulcra ejat. Qui ait at eam: ,() mulier, (fualis es et quare sic pendes?". At illa per miraculum Dei vina fuit ${ }^{1}$; comiti respondit: .. Nulier extranea sum a partibus longinquis. (uonodo luce reni nescio; Dens scit." l'omes vern ait: „Cuins equus est qui per ${ }^{3}$ arborem stat?" At illa: .Mleus est." ('omes rero intellexit ipsan generosam et pietate motus ait ei: „O domina bona, appares generosa. Tantum rnicam filiam habeo parunlam. Si mannceperis eam nutrire et docere, a peua ista te liberabo et "um hoc mercelem condignam a me recipjes." At illa: "1)omine, in quantum seio restram voluntatem adimplebo." C'omes vero eam de quercu deposnit et secum ad eastrum sum reduxit ac custodiam filie sne ei tradidit, et in eadem cameja, in qua et comitissa. iacuit habensque secum puellam; inter lectum comitis et imperatricis erat lampas semper ardens. Et tam honeste se gerebat imperatrix, ynod ab omnibus amabatur. Frat tunc temporis in anla comitis quidan senescallus, yni jmperatricem dilexit et ei de amore inordinato loquebatur. At illa: „sicias, karissime, quod votmu feci Deo, quod nunquam aliquem diligam tali dileetione, nisi quem teneor ex diuino precepto diligere." Ait seneseallus: .Tu nullo modo ris eonsentire?." Que dixit: „Quid iterum rultis andire? Totum quod feci firmiter tenebo." Audiens hec seneseallus cum indignacione ab ea recessit, eogitans in eorde suo: ,wi potero, de te vindieatus "ro." Acrilit rna nocte, unod ostium eamere eomitis apertum crat dimissum. Hoc cum vidisset senescallus de nocte et ommes dormientes inuenit, respexit per lumen lampadis ad lectum imperatrieis. Vidit dominam solam cum filia comitis insimul iacere. Longum cultellum extraxit et guttur puelle per medinm scindebat et sic cultellum sanguinolentum in maun donine ipsa neseiente ponelyat ${ }^{4}$, ac si diceret: "Cum dominus mens rigilanerit et per lumen lampadis cultellnm in manu eius singuinolentum viderit, sine aliqua hesitasione credet, qnod ipsa propriis manibus puellam interfecit, et sic ipsa mali morte (Fol. $\left.81 \mathrm{r}^{\circ}\right)$ morietur:" Puella vero fuando erat interfeeta of cultullus sanguinolentus in manu eius ipsa dormiente positus, cito comitissa est a sompuo exprergeficta et per lumen lampadis enltellum vjdit sanguinolentum in manu domiue. Her viso fuasi extra se posita «st; dixit comiti: "O demine, eito ad leetum domine respice et ridete in manu eins mirabile." ('omes vero expergefaetus a sompno at lectum domine respexit et. (quando cultellum sanguinolentmn vidit in mann eius, tubatus est, rndique ad eam rlamanit et dixit: "Vigila! Quid est hoc yuod video in manu tna?: Illa rero ex elamore expergelacta est, et cecidit cultellus de manı sua. Respexit juxta latus sum et puellam mortuam imuenit et totum lectum plenmm sanguine sensit. Statim ${ }^{5}$ alta voce clamanit: ,Heu mihi! hen! tilia domini mei est occisa!“ ('omitissa, mater pmelle, cum hee audisset, yuod filia sua mortua erat, ad dominum summ lanentabili roce clamanit: „Interficiatur ista mulier diabolica morte turpiswima, une nostram rnicant filiam occidit!" Tune comitissa ad imperatricem lornebatur: ,Aperte apparet. (quod puellam propriis manibns occidisti, fuia cultellum in mann tua hahes. cum fua filiam meam oceidisti. et ideo morte turpissima condempuaberis et morieris." Ait comes imperatriri cum cordis dolore:

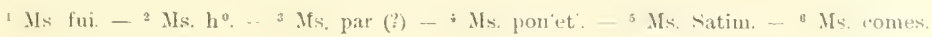


..1) muliner, nisi timor Dei esset, ("olpus tum statim cam gladio diniderem, eo quod te a sus-

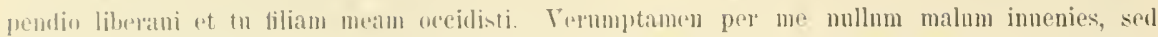
rito sine vlteriori dilacione extra comitatum meun pergas, et, si post istmm diem in terra mea te innenero, morte tnrpissuna condempmaberis." Imperatrix dolore plena surrexit et se induit restimentis ot sola palilidum ascondelyat ef rersus orientem equitabat. I'um sic in via equitassot, vidit ax sinistra parte patilulum altum, ad quod satellites rmm hominem anl suspendemdum dnxerunt. Imperatrix mota pietate perenssit equn enm calearibus et ad patilumum perrexit rt ait satellitibus: „'arissimi, parata sum malefartorem redimere, si pro vita eins meredem folueritis recipere." At illi: .Placet nolis, st pro vita cins solnas." Jomina com

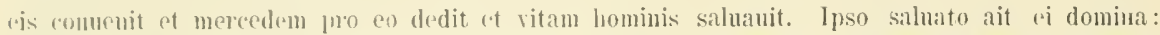

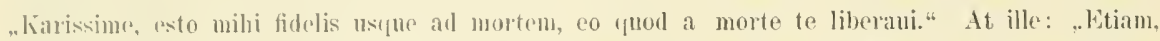
domina, hor tirmiter jumito tilis." Et sic secputus est dominam per viam. Cum autem prope rinitatem vencrunt, ait ei domina: .Preedas mo in cinitatem ef honestm hospicium pro me recipiats." Ille vero sie fecit, of in riuitate moram per aliçnos dies traxit. Homines illins rinitatis de eius nimia puleritndine admiralsantme at de amore inordinato ei loynebantur, sed mullo modo prenaluerunt. Aredid cito post hee, quod quedam nanis enm dinersis mereimonis in

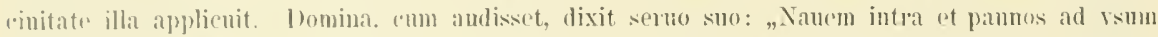
menm vide." Fammlus vero intranit et dinersos pannos presiosos innenit dixityne magistro nanis. of 'um domina sna loquerotur. C'oncessit magister; famulus precessit, demuncians domine de aluentu magistri uanis. Post hee eito magister intranit et dominam satis honorifier salutaut. lumina vero de pannis ad rsum smum sibi ferendis enm magistro' loquebatur. Qui concessit th nanem ascentit tamulusque domine cum eo perrexit. Ait ei magister: "Tili consilimm mem panderem, si in te confidere possem, et, si consilium menm celaneris, optimam mercedem a me

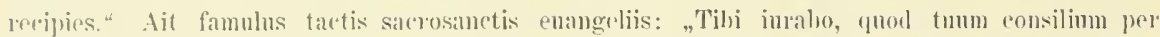

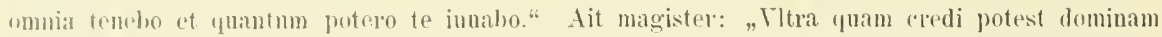
tuan diligo. 'T'anta pulcrindo in ua est, yuod ommia lrona mea pro eins anore darem, ut, si per te optimere potero. prete a me pnod rolueris et tibi dabo." Ait famulus: .Dic mihi qua via potero prodesse." (Fol. $\left.81 \mathrm{r}^{n}\right)$ At ille: ,Ad domium tuam perges et ex parte mea dicas: pamos mullo modo extra nanem exteudere rolo, sed facias vt nanem intret ijsa et non farjas ipsam intrare, quousque rentum valiclum joro me habuero. Tume eam ducere mecum volo." Ait fanulus: "Bonum pst ronsilinm tumm. Tha mihi mercedem, et totam roluntatem tuam adimplebo." Mercenle accopta acessit famnlus ad dominam denuncians ei, gnod magister nanis mullo modo

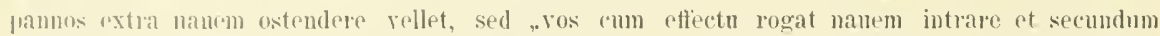
voluntatem vestram pannos at alia neeessaria poteritis optinere." Domiua vero eredens dietis lamuli sui ad nanem aceessit. ("un rero intrasset domina, famulus oxtra nanem se tonnit. Magrister veros, videns ventum validum, sursum rehm erexit et nangabat celeriter. Domina hoc

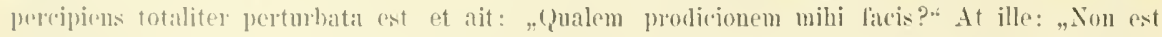
molicio. ynia te camaliter cognoscam el in rxorem muam dncam." At illa: "Totmm feei Deo,

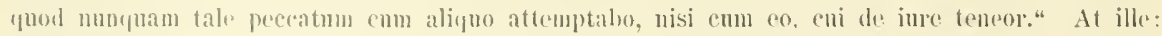
". Noli talia diecre! Jodo es in medio maris; nisi mihi consenseris, te in medio maris proiciam." 
At illa: „si ita est, locmm milhi in medio nanis prepara, ot tham voluntatem perficiam." Magister credens pi locum fecit preparari. losa vero curtimam intrant et thexis genibus iu hac forma Denu oranit: ,Lomine Dens mens, qui me de junentute mea rustodisti, custodi me in late hor:a ab omni pollucione, vt possin mundo corde animam tradere tibi. ()racione facta smrexit tam granis tempestas in mari. yuod nauis frangebatur et ommes periermnt rxeepta domina et magistro nauis. Lomina adbesit rni tabule, que ad terram eam duxit, magister alteri tabnle; nee domina de magistro scinit, nee magister de ea. Domima rero ad fuoddam cenolium monialium per jexit. in cuo honorifice snscepta fuit ot ibidem wre multa tempora tam sancte uilebat, puod gratian ej Dens contulit, quod infirmos simabat. Tude multi infirmi ex omni parte ad eam confluebant, et fama eius circumquaune diffusa est. Tune temporis frater imperatoris, viri sui, cui eam per crines suspendit, factus est vilis leprosus. Miles, ani filiam comitis occidit et cultellum sanguinolentum ipsa dormiente in mam eims postrit, factus est cecus et smdus. Famulus, qui decepit ram, factns est claudus. Magister nauis demens factus est. Imperator cum audisset, quod tau sancta mulier in cunadam abathia monialium fuit, dixit fratri sno leproso: „harissime, accedamns ad cenobinm monialium. rt a lepra tua per illam sanctam dominam curari poteris." At ille: .Vtinam a lepra hac essem curatus!. Statim imperator in propria persona cum fratre sno ad locm monialium perrexit. Imperator a priorissa querebat, si talis domina in cenobio esset, "fue infirmos curanit, et, si talis esset, rt ${ }^{3}$ ad eum reniret precepit. At illa: „Etiam, domine, inter nos manet." Ferit eam vocari, it se coram imperatore presentaret Imperatrix vero cum peplo faciem suam abscondit, ne de niro sno imperatore cognoseretm. Com ad eum renit. satis honorifice emm salutanit. Ait ei imperator: .O domina, scitisne fratrem menm a lepra sua cmare? si sic, pete a me quod volneris et tibi dabo." Imperatrix respexit circmonapue et vidit fratrem imperatoris lepra percussnm, militem, qui filiam comitis occidit cecum et surdum². hominem, quem de suspencione siluanit, podagra percussum et rermes ex ommi parte eius seaturiebant, et magistrum nanis dementem. Vude omnes cansa sanitatis arl ennden locm renernut. Tunc ait imperatrix imperatori: .Domine, si totum imperinm daretis milhi, restrmm fratrem ner alipnem alimm potero curare. nisi coram omnibus confessi sint." Conuersus imperator ad fratrem summ ait: (Eol. $82 r^{0}$ ) „'oram omnibus confitearis, it a lepra tha mudari poteris." Hle vero de totil vita sua est confessus, sed quomodo imperatricem per crines smspendit nilil loquebatms. Ait imperatrix imperatori: „Domine. libenter medicinam apponerem, sed in ranum, quia nondum est confessus plene." Imperator conversus ad fratrem summ ait: "Qualis est miseria tma in te? Nomne rides. quia vilis leprosus es? Confitearis rito, rel extra societatem meam te proiciam." At ille: „O domine mi, non potero confiteri, nisi mili prius constet de tna misericordia." Ait imperator: ..Et quid contra me deliquisti?* At ille: „Etiam, donine, magnam offencionem contra ros feci et misericordiam peto, antequam delixtum meum pandam." Imperator vero de imperatrice non cogitabat. eo quod credidit eam a multis temporibus esse defmetam. ot ait ei: „I)ir mihi, quid contra me deliquisti, quia sine dubio totum tibi remitto." Ille vero statim narranit, quomoro imperatricem ad peceatum solicitanit et quomolo eam per crines suspendit. Imperator etm hec audisset, quasi extra se positus, in furia magna dixit: „0 pessime, vindicta lei cecidit smer te! Si istud ante scinissen, moirti turpissime te condempuassem.3." Miles, qui tiliam comitis

Se mot manque dans te ms. - ' Ms. claudi. - ${ }^{3}$ Ms. cudepnassem. 
orcilit, dixit: .Nescio de qua domina ros lounimini. sed rala domina suspensa per crines fuit in suadium foresta et per romitem, dominmm memm, innental et ad rastrum ejus ducta, fue filiam eins ad nutriendum recepit. bro wro eam ad peccatmm solicitani et noluit mili concedere.

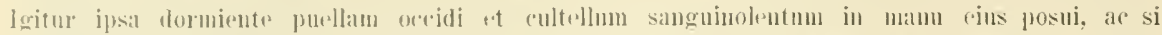

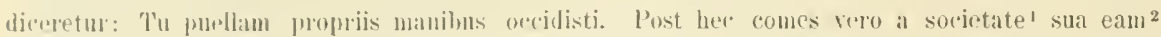
expulsit, sud quomodo postea vel quo init penitus ignoro." Tume ait latro: "Nescio do qua domina ros loquinini, sed voa pulcherima domina per viam equitabat sola. Ego vero tune temporis ad suspendendum ductus eran. Ipsa repo me a morte liberaut, quia redempcionem

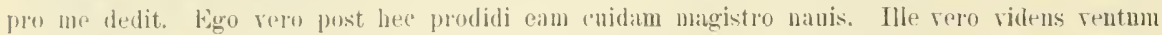
validum eam serum duxit, seyl quid actum sit penitus ignoro." Ait magister manis: „Talem pulcram dominam ar ganerosam mectum retepi et in medio maris cum ea commisere rolui. fpsa bero olarioni so dedit. Finita oracione nawis frangebatur in duas partes et ommes submersi sunt. Ego rero adhesi rni tabule ad terram reui. Guid actum sit de domina, rtrum sit smbmersa, penitus ignoro." Tume imperatrix alta nore elamanit: „Karissimi, ros estis pure conlessi. Iam apponam mediciunu." Vude omnes sanitatem receperunt. T'unc domiua coram omnibus faciem summ ostendebat. Inperator ram ean vidisset, noticiam eins habebat et ad (am rucurit et amyluxatus est eam et pre gandio fleuit licens: „Bemedictus Dens! lam inneni quod desiderani!" Juxit eam ad palacium cum magno iubilo et sic in pace vitam fininit.

(suit la Moralitas loj. $82 \mathrm{r}^{0}-\mathrm{r}^{0}$ ).

\section{D}

\section{De pudicitia et tolerantia cuiusdam imperatricis}

Ma. Paris, Bibl. nat, f. lat. $14+63$, fol. $36 \mathrm{r}^{0} \mathrm{a}-38 \mathrm{r}^{0} \mathrm{~b}$ anc pagin.: fol. $32 \mathrm{r}^{0} \mathrm{a}-34 \mathrm{r}^{0} \mathrm{~b}$ ).

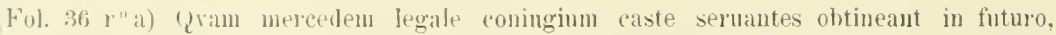
nondum, tratres karissimi, foguoseimus, stexpectamms. Virumptamen in presenti multis niolentian coningii rospuere profuit, quamuis multa nikerntm snstimuisse aduersa. Que et quanta susimm, uxor Ioachim, al, impulicis sembus ot talsis judicibus passa est obprobria (t qualiter

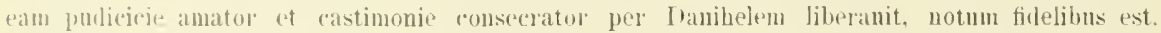

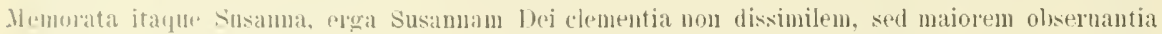
susiunf in rogutionm profutuam tam presentibus quam futuris cuiusdam mulieris nirtuten simni ret tolenutian proforimus. Relatione preteritorum, harissimi, que non nidimus recolimus

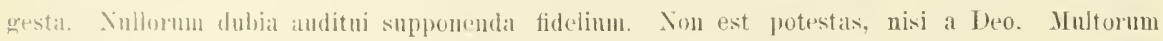
igitur temporum elapse currioulo atque per orbem religionis crescente uehicalo a principe

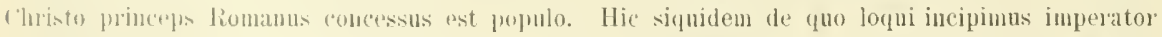

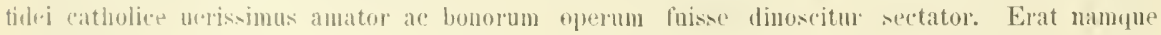

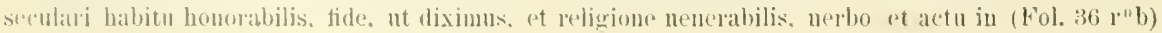

W. soritate. - etam menque dans le ms. 
regno amabilis. Cuius tantis honestatibus niri clarissimi, ut congrum fuerut, pronidetur Dei clementia uxor genere et moribus nobilissima atrue puleritndine multum honestissima, et imperatori coniugali et nuptiali more copulatur. Non illicita dilectio neque turpis carnalisque deleetatio illis adherebant, sed ad innicem sobrie et iuste sub legali coniugio sp non mediocriter filigebant. Cum itagne res publica sub talihus et tam insignibus personsis sullimaretur censu et omni prosperitate, nenit in mentem imperatoris, ut pergeret longe lateque per orbem uisitare loca sanctorum. (quod antrm habuit din in cogitatione in congruo tempore implenit opere. Accepto itanue a nenerabili coninge sua tam longi itineris assensu imperator nobilissimus, sumptus nie sufficientes multosique nobilium ac familiarum proceres secum assumens, regnum et quendam fratrem snum adolesenten nenerantissime imperatrici accentius commendans, digressus est. ('umpue illa regni alta et humilia, maxime ecclesiarum iura, Leo opitnlante uiriliter regeret, iam dictus sui domini frates, specie nenerabilis regine deceptus, in eits amorem vehementer exasit. Imperatrix nampue rogatu et amore imperatoris illum tenere diligehat ignorans quod ipse in pectore incendium gerebat. Crescebat namque in innene illicitus amor, ita ut sommn cibumque anferen: unltum illins monstraret nalde aftlictum. Thue nero familiarins et frequentins intendere in eam cepit coactusque (tuo stimulo urgeretur, qno igne ureretur tandem regine apernit. Qno audito, quanuis nou modice subdentur molestie, aliter in animo gerens non minace responsione stultum inuenem terruit, dicens tale obsequium al, (Fol. $36 \mathrm{r}^{0}$ a) alia non a se ei exhibudum. Et quia fortis est ut mors dilectio, cepit maiori flamma imenis urgeri et toto corpore nehementer attennari. Regina nero, intra se sapienti usa consilio, iuueni cecato ad! eius amplexus se nenturam esse promisit. Illa tamen fugiebat locum tantique sceleris factun. Interim quoque parari turyim quandam non distulit, in qua dnos adolescentes et duas pnellas ad seruiendum iutueni mittere cmranit, nt uecessaria illis queque sursum cum funibus traherent. leterminato quipje die, qno tmrim illam imperatrix et innenis miuatim conscenderent et quod ipse desiderabat expleret, ganisns est. Cum nero competess hora fuisset, preennte imene usque ad hostium turris ascenderunt gradus. Quo intrante, clauso hostio pndica et renerabilis domina in domum rediit retento iunene in turre. Sedit Roma pacifica et omne regnum eius non decli. nauit imperatrice regnante, quousque imperator rediret ad propria. Transictis nero quinque aunis, ece quidam nenerunt, qui imperatorem uenire dixerunt, aliiqne et alii post eos in proximo imperatorem nuntianerunt. Qnam leticiam ynantume gandium de aduentu domini sui imperatrix habuit, eins hylaritas unltus demonstranit. Nobiles confestim et maiores cleri inssit secum in occursum imperatoris exire et cinitatis nicos ornamentis parari decenter. Fratrem nero imperatoris inconsulte de turre foras emisit, nt obuins fratri exiret. (qui in occursn imperatoris reginam prenenit atque eam uersa nice male et inconnenienter diffaminit. Requisitus a fratre imnenis, cur tam aftlictus corpore et pallidns facie atque crine deformis esset. respondit cum lacrimis: "Mi frater karissime. pessima coniunx tua et pessima meretrix, sicnt cum multis lornicari (Fol. $\left.36 \mathrm{r}^{\circ} \mathrm{b}\right)$ consuenerat, me ipsum precipitare in seelus secum temptanit. Nullus enim ab eins inpudico amplexu, ex fuo recessisti, prohibitus ext. (ynod terre non potui. sed ab ea recedens in quadam turre me numc usque inclusi. Igitmr maiestatem imperatoriam impia meretrix quam usmpanit ne osculo et fumiliari colloquio contaninet cauendum est." Audiens itaque imperator quas non speranit in uxore nobilissima contumelias unntiari, dolore cordis intimo pereussus pene exanimis corruit in terram. Receptisque animi uiribus post spacium 
mius hor surexit est ronlontatus personarum consolationibns equm ascendit. In crastinum ure imperatrix nobilisima r.m comitatu nobilimu et tubis einitatis peruenit ad uirum sum. Que rum illum, ut diximn, ut dignum erat, amplecti ut oseulari nolusset, perensia graniter ab imperatore in

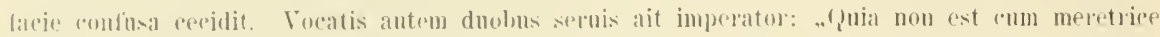
cortandum, ite in siluan profundam et dneite illam ad derollationem?: Prob dolor! Imocens

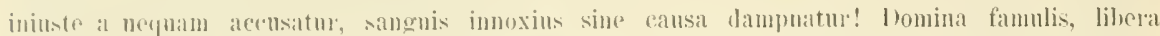
seruis traditur, nobilis ah iguobilibus dueitur, ut gladio interimatur! c'nmone ad bocmm. nbi

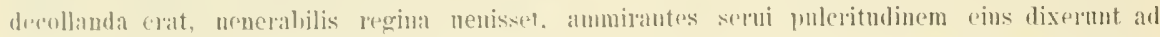

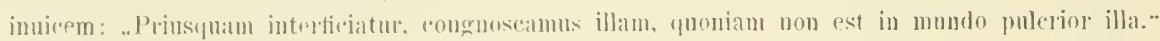

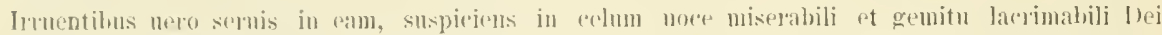

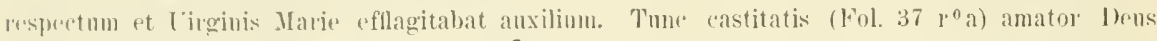
ut aniea prodicicio L'irgo Maria nou distflerunt anxilium nittere illi. Non longe quippe iter

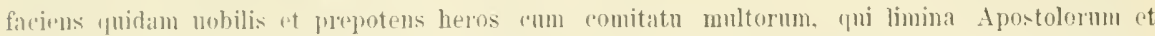
loca sanctormm Rome nisitanerant, audientes tantis clanoribus resonare silnam. primo putanerunt alipuam leram in retibus corrusser. neloci cursu illne usque permenerunt. Iam nero pre nimio

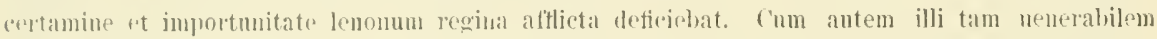
personam seruos impudicos opprimere nidiscent, morti ens lestinanter tradiderunt. Reguiritm legina diligenter ah eis, quenam esset mulier et quare talia contigisient ei. c'elata nempr dignitatis altitudine, smppliei uultn. humili noce roganit, nt eam domins rorum secum duceret

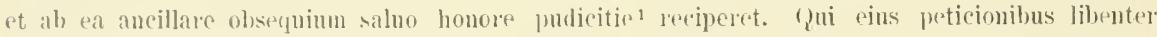
ammens ad propria perduxit illam. (puan itaque uxor eins honorifice suscepit atyme procul Juhio unirnm filium educandum sili commisit. Suscepto igitm parunlo illa maiorem adhibuit diligentiam yutum si ipsa cum genuisset. Vitabat quicenit erat senrilitatis, amabat que erant honestatis. fugiebat laseiniam, frequeutabat crelesiam. (), quanta est hostis calliditas, sed maios (nt montis et corporis eastitas! Non alter, sed idem maligms spiritus et fornioationis anetor istam, de qua agimns, temptare non desiit, sed nineere nullo modo ualnit. Erat quidam miles in 'nria. yni al amorem huins mulieris pertingere cupiebat, quam bandis sermonibus multisqne pronissionjbus, ut se sibi in coniuginm copmlaret, (Fol. $37 \mathrm{r}^{\circ} \mathrm{b}$ ) temptabat. Ipsa nempe se enm eastr diligure respondens anore domini sui. cuins erat frater, nubere nero ei omnino neganit. (quan ob rem intraut sathanas in enm. Fx tunc trartare cepit miser in animo, ynid mulieri.

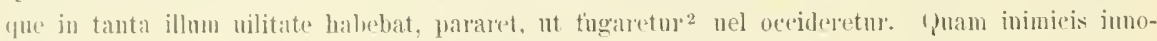
centie odiosit fit sempor libertas! Congregatis impins ille in rorde sno multis sceleribus quod nequins erat adnersus preclictam mulierem regit facere. Intempeste igitur noctis hora nenit ductu diabolico ubi uruerabilis mulier illa dermiebat ot intans in sinu eins sibi commendatus puerique. filii fratris sui, gnttur 'um cultro per medium sicanit; gladium quoque in mann mulieris deruniantis relinguens resessit. Eece fratris f'ayn fratrieida seeundus! Xon minus nequam, sed manior chayn fuit isto! ('ayn innidia deceptus solum occidit fratrem: iste fratris filium, ut imnoxia of pndien muliey oecideretur. ('ayn innocentem fratlem in die occidit in agro; iste

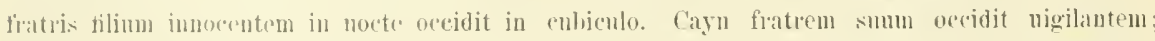
iste filinm fratris oecidit domiontem. Prom omia iste peior cayn reputatus. ('um nero oceisi

\footnotetext{
1 Iss. puditicie. - ${ }^{2}$ Ms. fug'et
} 
pueri sanguis per lectum decurneret et latus mulieris inficeret, rxitata nehementer expanit. At illa cum se miseram noce lacrimabili prockamasset, sollicita mater de filio et pater pueri surgentes concite de lecto cum lumine nenerunt ad filium sum. Videntes anten iugulatum fmerum et cultrum in manu mulieris, quis dolor quantusqne luetus innaserit pos et ommem fimiliam nix explieari potest. Tenit denique sueleratissimus homicida, fictas fundms lacrimas, et noluit oceidere tanti sceleris ignaram, Fol. $37 \mathrm{r}$ "a) dicens frani suo: "Adduxisti hne meretricom in multis criminibus in alia patria deprehensam et inste olim indieatam interfici. It igni iam tradatur, mors filii tni dignam eam iam facit." Pars populi nequissimo fauchat, pars altera laterimosis clamoribus condolebat. Heros nempe nobilissimus et nxor eius uenerabilis cam interfici prohibentes, ad portum maris proximi nantis, nt in alienam teram dneerent illam, mandauerunt per seruos. Venit ergo miserabilis, perfusal faciem lacrimis, honombilem comam discerpens, cum sernis ad mare et intrauit nanen. cumpne per altum naute naugassent, considerata eins specie ac unltus pulcritudine, nt eis assentiret, illim allocnti snut. Resistentique illi ob pudicitie amorem dixerunt nante: „Tnum elige e lnobus: ant nohis commisceri, ant in mare precipitari." Responditque mulier se libentins inter fluctus maris deficere quam eorum libilinosis peticionibus assentire. Qui mutato consilio in quadam alta rupe, que ante eos in mari eminebat, Deo prouidente eam dereliquerunt. c'umque totam ibi noctem duxisset insomnem fidelis et Deo dilecta mulier. summo diluenlo merore et tridnano iciunio nimis afflieta obdorminit parumper. Apparnit antem illi persona puedam in mulieris specie, ammirabili facie. oculorum stellanti acie, nocata Regina Glorie, dicens ad illam: "Quia multa propter fidem casti coningii obsernanlam sustinuisti aduersa, amodo omnis a te impiorum temptatio expellenda est et que passa es iniurie manifestabmutur. Collige ergo herbam. que sub capite tno est. et quibuscumque leprosis bibere in nomine lomini donabis sanabuntur." Excitata itaqne nenerabilis domina et de nisione letificata, mu denotis orationibus herbam collegit, cui similem ante non niderat, atque cyrothecas de manibus sus extrahens implenit. Hora igitur tereia, quasi a lleo (Fol. $37 \mathrm{v}^{0} \mathrm{~b}$ ) missi, iter facientes naute iuxta rupem illam nocatique ab ea applicnerunt. Videntes autem illam tam nenerabilem esse personam condoleutes ei receperunt eam in namem et duxerunt al portum. Egressa nero de naui inuenit quendam leprosum nisu miserabilem, attritamque herbe portiunculam cum nino dedit leproso bibere. qui confestim sanatus est. Quo audito neniebant ad eam ex ommi prouincia illa leprosi potatique poculo herbe sani et in olmmes renertebantm ad mopria. Exinit ergo fama hec longe lateque nenitque ad cinitatem illam, in tua erat ille impius, qui fratris sui filium iugulanerat in sinu huius domine, leprosus effectus. Requiritur igitur mulier illa nenerabilis adduciturque multis precationibus denicta ad egrotum. (quam nullus familie domus nec ipse nequan cognouit. Rogatur illa obnixe at hatre leprosi, ut ei subueniat et quiceuid mercedis inde nellet acciperet. Ipsa nero se illi subnenire promisit. si confessus de ommibns pecatis prins coram ea et septem personis fherit. c'nmque ad confessionem ille leprosus uenisset, celans scelns, quod in ipsa commisit, multa dixit. Responditque mulies nil proficere medicinam suam aluscondito ullo crimine. Thne ait trater suns, cuius filium interfecerat: „Aperi. thater, cor tumm et emmuda ab omni peccato, et, si aliquid adnersum me male egisti, totum tibi dimittatmr." Resolutus denique, in lacrimis qualiter egerat manifestauit. Heros antem andiens expanit nehementer, dicens se plus de amissione nobilissime mulieris quam de morte filii tristem esse. At illa respondit: „Ego quidem, domine mi. sum illa mulier, que non reddam malum pro malo, 
ser homum pro malo retribuam." Restitutoque jllo sanitati rogahant, ut ibj remaneret et acci-

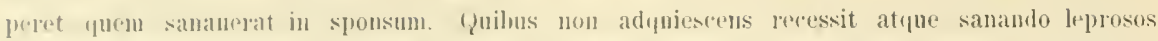
nenulles Romam incog(fol. $38 \mathrm{r}^{\text {a) }}$ nital potiit. Mansit itaque per aliquot dies Rome, mundans a lepra plurimus, et non cognouerunt eam. Lumarrat itarue fratrem impratoris uleisente beo

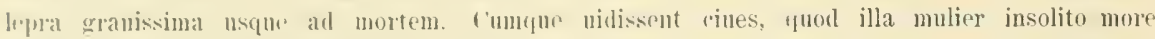
medirorum multos sinaret a tam graussima infirmitate, nuntiauernut imperatori adess quandam f'minam, que fratlis sui posert lingare lepram. fianisus ereo imperator inssit eam adduri ad se.

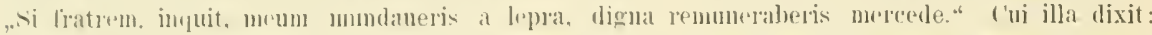

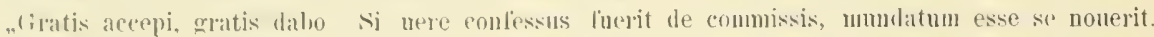
contiteatur mihi coran pilpa at senatu Romano quibus subditur cupjos, si sinns esse desiderat. sui antem omnino forporis sanitate earebit." Tunc inuphis infirmus a ciremstantibus rogatur omnilus, nt nichil ahseonderet pereati. It ille qualiter aduersus imperatricem iastam et per onnia landabilen corat ondine manilestanit conlisus. Tmu injerator Hens et pugnis faciem pertusque perentims miserum se exclamaut. Flebant autem uni aderant et qui andierant boe nribum recordatione imperatricis egregie. At illa non sustimens fietus illormm et suspiria, sanato

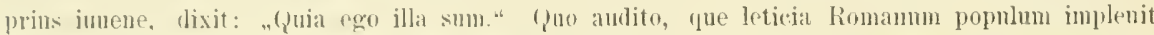
non est facile dicto. Voluit rero imperator eam quasi a Domino resuscitatam in nxorem, et pupulus in dominam accipere. Sed illa alites intra se agens dixit ad papan: . Yotum, domine, feri in tribulationibus meis I homino me ulterius ulli non roniungere niro, sed monachilem induere unstem at sernare eastimoniam. Requiratur roo anima mea de mam tua, si timens nel diligens hominem plus quam Demu beste saneta non melaueris me." Tune sanctus pontifex sine mora bsuedicens eam tradidit illi religionis hallitum. que, (quia pu(Fol. 38 rob)dicitiam seruauit, feliciter ad eternam uitam migranit. Amen.

\section{$\mathrm{E}$}

Version du Miracle latin

Ms. Londres, Brit. Mus. Harl. 2316, fol. $6 \mathrm{r}^{0}-7 \mathrm{r}^{0}$,

(Fol. $6 \mathrm{r}^{\circ}$ ) Fuit quidam Romanms imperator, (ni prouddit Dei elementia rxorem genere ct moriłns nolilissinam ac corporis puleritudine honestissiman. Inter los castus amor roniugii scmper tuit. Acrepto itacue assensm a coninge longe lateque per orbem limina sanctorum visitare disposnit. Regnum autem et quendam fratrem snum adolescentem eidem tradidit. l)ichs adolwsens, sperie imperatricis derptus, in eins anosem rehementer exarsit tandemque impreatrici apuriens pluries fidem molestns fnit. Ipsa semper renut. Tanden iuneni cerato ad rius amplexus venire se simulate promisit. Interim turrim quandam parauit; duos adolescentes

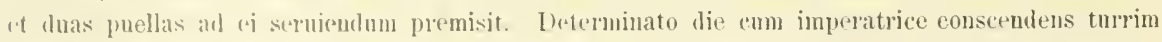
illam et preferens atam inclusus est eaute ah eadem. Sedit igitmr sub pudira imperatrice Roma pracifica, donec post quinque annos imprerator rediret ad propria. Audiens eius aduentum 
vieinm ganisa pst et cum nobilibus et maioribus ei obuare disposnit. Sed inconsulte turrim aperuit, rt fratri sno adolescens obniaret. loitur statim reginam preneniens eam versa vice turpiter infamanit. „Mi hater, inçuit. karissime. me ipsum coniux tua in seclus adulterii precipitare attemptanit. Immo, nullus ah eius impudico amplexu, postquan recessisti, prohibitus. (quod ego ferre non potni, sex ab ea recedens me in quadam turi conclnsi." Hec et humsmodi cum requireretur a fratre, quare tam pallidus ot eriue deformis assot, narranit emu lacrimis. (quo andito imperator in terram corruit pene ex(Fol. 6 ronanimatus. In crastino emm magno conitatu venit imperatrix ad rirum sum. Q Que, com illum, vt digum rat, rellet amplecti, percussa ab eo in faciem cecidit. Vocatiscue duobus seruis imperator ait: "Quia non est cum meretrice certandum, ite ad siluam profundam et. ibi eam decollate." ('um peruenissent ad locum, admirantes eins pulcritudinem dixernot ad inucem: "' 'nm sit omnium pulcherrima femina, priusquam interficiatur, fruamur aa." Que roce lacrimabili Thesn et Virginis Matris, vtpote totaliter destitnta, imploranit anxilinm. Contigit antem istis existentibns nobilem quendam, qui Rome limina Sanctornm visitanerat, pertransire. Hic mulieris clamorem andiens statim colerimo cursu ad eam cum comitatu pernenit, videntesque seruos impulicos tam nobilem persouam distrahere morti eos concito tradiderunt. Requirunt the esset at cur ei talia contigerunt. Illa celata dignitate voce humili rogauit, vt dominns eorum secum ram duceret et in obsequium ancillare reciperet pudicicie honore sibi salno. Qno amnente et ad propria perducto var. domini eam honorifice recipiens suicum ei filinm educandum commisit. Illa paruulo suscepto maiorem diligenciam adhilnit (tuam si eum genuisset. Fngiebat lasciniam, frequeutabat ecclesiam. Erat igitur in curia miles tnidam, frater domini, qui amore eius succensus crebris allocncionibus innitabat al malnm et sibi coningio copulare temptabat. Illa vero caste se eum diligere amore domini sni, sed ei nullo modo posse mbere respondit. Qutim ob rem cepit cogitare juo morlo de ea, ynam enm contempsisse putanit, vindicare valeret, vt vel oceideretnr vel fugaretur. Itaque intempesta noctis hora dnetu diaboli venit vbi illa dormiebat et infans in sinu eius sibi commendatus, guttur(pe pueri, filii tratris sui, cultro per medium secanit, gnem in manu mulieris dormientis relinquens recessit. Illa igitur, cum sanguine tincta esset ad latus, excitatur. Stupet de sanguine, miserando proclamat. Pater et mater de filio vnico sollieiti statim accurrunt cum lumine. Filium vero ingulatum videntes et in mann mulieris cultrm snbito dolore percellnntnr. Inmo et familia tota cum eis renit, deniqne sceleratissimus homicida fictas lumdens lacrimas, rolens ram statim occidere de serere nescientem, dicens fratri sno: "Adduxisti hue pro multis criminilus in alia patria interfici indicatam. Igni iam tradatmr, que filium tum nnicum interfecit." (Fol. 7 ro) Nolílis vero vna cum vxore ean interfici prohibuerunt, mandintes nantis ad portum proximi maris, vt eam in alienam terram ducerent. Venit ergo lacrimis perfusa faciem comamcue decerpens cum seruis ad mare, cumque in altum maris cnm nautis venisset, capti eius pulcritudine, vt rei nepharie assentiret, eam allocuntur. Resistenti ob amorem pudicitie dixerunt: "Tnum e dnobus elige: ant nobis commiseri, ant in mare precipitari." Illa libencius fluetus eligit. Qui mutato consilio eam in quadam rupe alta, que in mari coram eis prominebat, iutactan dinine Pronidencie reliquermut. Thi rum totam noctem duxisset insompuem', merore et tridnano ieimio al'flicta ${ }^{2}$ panlulum obdorminit. Et ecee Beata Virgo Maria, regina glorie ${ }^{3}$,

\footnotetext{
${ }^{1}$ Ms. isopne. - 2 Ms, aftlictam. - ${ }^{3}$ A cet endroil, he ms, donne e'n margr: consolacio marie.
} 


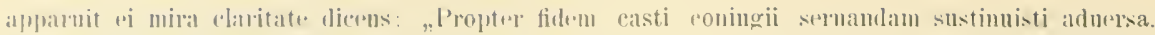

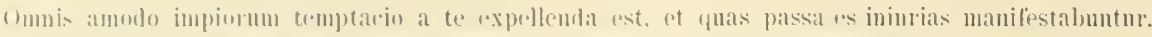

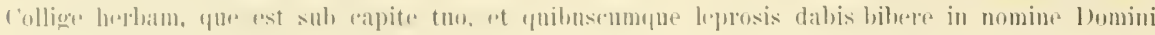

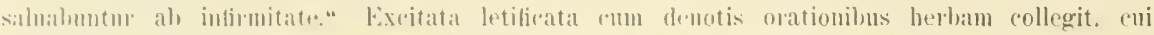
similem numpatm viderat, diontexasplur de manihus extrahens implenit. Hora antem tertia naute

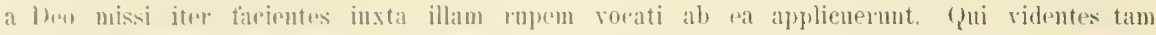
remerabilen ense personam rompacientes reneptam in nani reduxerunt ad portum. Egressa de

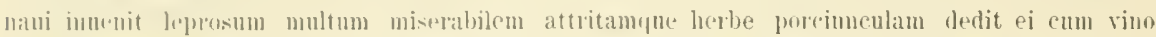
bihere. statimulue sandus est. Fama ipsius vodiqur dimulgata venit ad ciuitatem, in qua erat

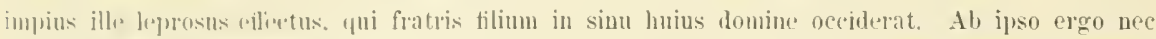

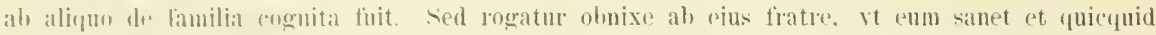

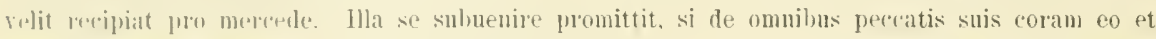
mprem personis ronfessus luerit. Hlo igitur multa namate, sed illud scelus penitus reticente.

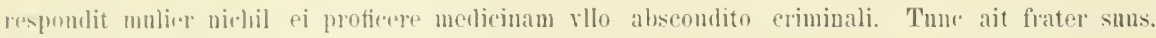

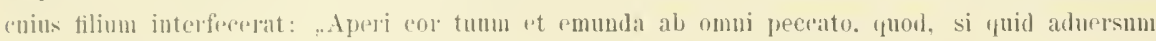
me male "rgisti, totmm tibi dimitto." Resolutus in larrimas expressit quod fectrat. Heros expauit dicens se plus de amissone nobilis mulieris quan de amissione filii tristem esse. (que respondit: "Inonine mi, egor sum illa mulier: que malum pro malo non reddam, sed honmu." Restitutum santati optulerunt in sponsum, sed ipsa hec renuens sanando leprosos liomam putiit et ab (Fol. $\left.\tau \mathrm{r}^{\circ}\right)$ omnibus incognita illue venit. Fratrem proinde imperatoris lepra inuaserat, quem pecratum summ conliteri coram papa et senatu Romano coegit rumque curauit. Quo audito imperator flens pugnisqur faciem suan cedens miserum se exelamanit, tlebantque ommes qui aderant recordati pie improtrjejs. Tunc illa Hetus eorum non sustinens eonfessa est: "Quia ego sum." Imperator joitur popmlnsine Romanns smmmo perfusi gandio imperatricem denuo tanquam a leo redditam assumere voluernot. Tnne illa dixit ad papam: .Totnm feei in tribulationibus meis lomino me nulli vlterius coniungi virn, sed in monedali reste castimoniam seruare. Rerpiratur igitur anima mea de manu tua, si dilicgens rel timens hominem plus yuam leum reste sancta me non velaucris." Thunc ille mox eam benedixit et induit saera reste, in qua et leo spiritum rerldiclit.

\section{$\mathbf{F}$}

\section{De imperatrice miraculum}

Ms. Paris, Bibl. nat, f. lat. [7.191, fol. $155 \mathrm{r}^{\circ} \mathrm{a}-157 \mathrm{r}^{\circ} \mathrm{b}$.

I

(F. $\left.155 \mathrm{r}^{\circ} \mathrm{a}\right) \quad$ Fnit olim nir (fuidam nobilis,

Vrbis Rome dux venerabilis, fisle constani et facto stahilis, Inxta causam index immobilis,
II

Vultu placens, nerbo compositus. chens, prole pre runetis inelitus, Legi semper dinine deditus,

Vite cursum agens dininitıs.

11. 2: Is Censu (le m.. Bibl nal. f. lal, 23333 d domne la bomne legron). 
III

Tali niro tam elaro genere

Heret uxor legali federe,

Que dinino rigata mmere

(F. $\left.155 \mathrm{r}^{\circ} \mathrm{b}\right)$ Mollem sexum uincebat opere.

\section{IV}

Illa virmm sequens per ommia Par in factis erat et gratia.

Tales innxit Dei elementia, Pares enim decebant paria.

V

Pietatis amaloant studimm

Restringentes carnis incendinm;

Relenabant pressmas flentimm

Peregrino pandentes ostinm.

VI

Frequentabant limen ecclesie, Snecmpelant fratrum inopir; Prompti semper ad opus gratie

Nil agebant sub falsa specie.

\section{VII}

Sie trahebant equali copula, Quos ducebat celestis regula; Quan seribentes in cordis taluala Saprienter tractabant singula.

\section{VIII}

Sic bonorum currente linea

Laborabant in Dei uinea;

Triturantes in cordis area

Sequestrabant gramm il palea.

\section{IX}

Dum sic agnut cuncta sollicite Incedentes angusto tramite, Voluntatis sagitta subite Pium unlnus fecit in comite.
I

Cogitalat paterfamilias

Causa noti pergendum alias

Visitare patrum ecelesias,

Paruijendens regni diuitias.

XI

Sic affectans comes diutins, sibi tantum hac in re conscius, Satagehat impleri citins, (quod nxori non erlat longins.

XII

Manifestat dux conscientiam ('ordis sui pandens historiam. Illa, canse landans memoriam, (F. $155 \mathrm{v}^{\circ} \mathrm{a}$ ) P'eragendi donat licentiam.

\section{XIII}

r'omes ergo paratis ommibus Fratrem summ fouct amplexibus, Quem relinquens in sponse manibus Gandens ibat amicis Hentibus.

\section{XIV}

Abeunte duce cum gandio sponsa sedens in regni solio Plebem ducis regit imperio; (randet omnis sub illa regio.

\section{XV}

Imperaluat henigne popnlis Secum habens germanum cousulis, Qui coniectis in ipsim oculis Venenatis pulsatur stimulis.

\section{XVI}

Vulneratus ipsins specie Sompno caret et noctis recunie; Caro pallens et liacta macie Argumentum probat miserite. 
XVII

Tali miles oppressus mulnere Premebatur occulto pondere;

Tamdem lorm proponit ynerere,

It hee possit illi detegere.

\section{XVIII}

Nactus ille locum collocuuii

sui modum narrat incendii.

llla nero mater cousilii

Hoe dicendum precipit alii.

\section{XIX}

Illuun pungit talis respousio;

sed lue, pacis usa consilio,

Sie promittit sul breni spatio

Subierturan eius obsepuio.

\section{IX}

Sic demulcens illius animum

Et pertractans iutra se plurimum.

Arte quali saluaret proxinum,

lormm turris inuenit optimum.

\section{X.S}

Thi lectum precepit fieri;

Eligmutur of dho pueri

Et puelle rinsdem numeri,

(F. $155 \mathrm{v}^{\circ}$ b) Qui ministrent precepto miseri.

\section{XYII}

Adest dies promissa militi;

T'urem intrat tenax proprositi.

Sirl matrona mellor illiciti

Thurem tiruat, spem fraudans debiti.

\section{xilli}

('lausus ucro miles anyueritur, Fame. siti, dolore quatitur, Paruo namene eilso refieilur, ()ui per corlam illi poryigitur.

XVII, 1: Us opp'sts. - XXX, 2: Ms. Dum.

\section{XXIV}

I ispensatur sic uita militis.

l'ost hec annis quinque preteritis

Narrat fama regressum comitis;

Gaudet sponsa non impar meritis.

\section{$\mathrm{XXV}$}

Hymnos eius uix possem serilere, Que nocato regali genere

In oceursinm festinat pergere,

Fratren ducis tollens de carcere.

\section{XXY1}

Sic egressus satis accelerat

Prenenire, ut fratri referat

Hact: sponse, non sicut egerat; Sic assistens ad fratrem properat.

\section{XXTII}

Videus illmm comes exhorruit,

Illi tamen amplexus prebuit Querens, cur tot pressuras habuit. Fraudem suam tumc flens aperuit:

\section{XXVIII}

"Sponsa tua nu causa Teneris Impetelat, se prebens ceteris, sed, dum spreui fetorem sceleris, Me concludit in fundo carceris.

\section{XXIX}

Eece nenit stipata ducibns Fruitura tuis amplexibus. sed ne credas eins sermonibus, Mos est enim fallendi talibus."

\section{XXY}

Tum referret iniquus talia, 1)ux turbatur, fremit insania; Vera credens fratris mendatia (F. $\left.156 \mathrm{r}^{\circ} \mathrm{a}\right)$ ll llxorem flectit conuitia. 


\section{XXSI}

Post hec uenit uxor et sotii, Quam dux cedeus pro culpa nitii Mortem inbet subire glatii Intra nemus illius predii.

\section{XXXII}

Inplent serui ducis imperimm, Dacunt illam in silue medium; Quam uidentes formosam niminm Accenduntur ad adulterium.

\section{XXYIII}

Festinabant illi communiter ('onmisceri cum illa turpiter, Sed matrona resistit fortiter Dei IIatrem nocans suppliciter.

\section{XXXIV}

Dum sic paguat notis et niribus, Viri quidam de Rome partibus Venientes illis tramitibus currunt illue moti clamoribus.

\section{XXYV}

Primum quidem credebant hestiam Sustinere retis molestiam, Sed nidentes talem ininriam Seruis cesis seruant innoxiam.

\section{XXXVI}

Illa gandet pro tali gratia Orans duci secom ad propria; Qnod dux implet motus clementia. Osculatur ducis nestigia.

\section{XXYYII}

$\mathrm{Vxor}$ ducis pretendens gardium Hanc suscepit in coutubernimm
Et affectans eius obsequnium

Illi tradit alendum filium.

\section{XXXVIII}

Nuttrit illa sollerter paerum Gratum nisn, etate tenerum; Sed antiquas inuentor scelerum Nichil agit erga nos prosperum.

\section{XXXIS}

Hostis, inquam, hmuani generis, Persequtor huius mulieris, Hane temptanit per fratren proceris, (F. $156 \mathbf{r}^{\prime \prime}$ b) Quem amabat comes pre ceteris.

XL

Temptat illam miles in plmilus, Hanc demulcens donis et precibus. Que cum nollet parere talibus, Miles tristis armatur fraudibus.

\section{XLI}

Intrat hostis in mentem miseri, Quem accendit ad mortem pueri. Qui nocere nolens mulieri Homicida non timet fieri.

\section{XLII}

Nocte surgit miles de lectnlo, C'ultrum portans accedit clanculo Vbi iacet illa cum parunlo, Quem infigit nepotis ingnlo.

\section{XLIII}

Thne discedens peracto scelere (ultrum infert muttricis dextere. De cruore tingens sub latere Exclamanit: „Hen, mihi misere!“

XXXIT, 3: Ms. illi; le ms. Bibl. nal., f. lnt. 2333 d donne: illis. - XXXIV, 4: Ms, motis; le ms. Bibl. nat., f. lal. 2333 A donne: moti. - XXXVIII, 1: Ms. filium; le ms. Bibl. nat., f. lat. 2333 A offre la bonne legon: puerum. - XXXVIII, 3: Is. in ueutre; la bonne leçon est dans le ms. Bibl. nat., f. lat. 2333 A. - XL, 4: Ms. armauit; ms. Bibl. nat., f. lat. 2333 A: armatur. - XLI, 4: Ms. Nomicida. - XLIll, 2; Mfs. effert; le ms. Bibl. nat., f. lat. 2333 A dome: infert. 


\section{$\mathrm{Xl1}+1$}

Id hat nocem dux et familia

f'urrunt illur lucerua prenia,

Qui comperta tali nequitia

Vlukatu replent eonfinia.

\section{XLF}

'lune prerumpens miles in medium Fratri sno dedit consilimm,

It dimpnetur lare per incendium,

bit hee landat pars malignantinm.

\section{XLTI}

Comes nero per patientiam

Fratıis sui dampuat sententiam;

Mandat nantis propter institian

Hanc proferre extra prouintian.

\section{XLVII}

Intrat nauem pallens tristitia.

Ducunt illam naute per maria,

(yui decepti decoris gratia

Commouentur' grami luxuria.

\section{XLVIII}

Illam tali petmut alloquio:

"Nostro satisfac desiderio!

(pood si non nis, talis est ratio,

(F.156 $\left.\mathrm{v}^{n} \mathrm{a}\right)$ lt iacteris in ponti medio."

\section{XIIS}

Illa malleus mortis perienla

T'ali non unlt fedari macula;

Serl mutantes hi commenticula

Hane relinyust in yuadam insula.

\section{L}

lbi mancus per noetis spatinm

Nullum bahet flendi remedium;

Tamdeu ithi supponens brachium

Tale uidit sopita sompnium.

\section{LI}

Illi quedant apparet nisio, Dei Mater in nultn regio, ('omparandis florenti lilio, Fonens illam tali colloquio:

\section{LII}

"Iam munc aura cessabit tubinis, Hec est meta tui certaminis. Tempus adest metendi seminis, Qno pandentur factores criminis.

\section{LIII}

Iam unuc surge, dilecta filia! Tna Lens nidit opprobria.

Huins herbe carpe uirentia;

Valent enim ad lepram folia."

\section{LTV}

Surgens illa summo diluculo Signat herbam erucis signaculo, (uam elenans abscondit sedulo, Cyrotechas habens pro uascnlo.

\section{$L T^{\prime}$}

Thi diu morata femina Ecce nanem per maris flumina Adducebat celestis Domina; Sed nautarm nescinit nomina.

\section{LTI}

Tidens illa nanem concurrere Ad se uiros facit comertere, ()uam tollentes cepto itinere Peruenermut ad portum libere.

\section{LVII}

Trune egressa mater eum gandio Qnemdam egrum nidit in binio, Qnem a lepre sanat contagio; (F. $156 \mathrm{\nabla}^{\circ}$ b) Tantum herbe nalet confectio.

XIJIX, 3: Ms. Si; le ms. Bibl. nat., f. lat. 2333 A donne: Set. - L, 3: ibi manque; restitué d'après le ms. Bibl. nat., f. lat. 2.333 A. - I. Y, 2: Ms. nauis; le ms. Bib. nat., f. lat. 2333 A donne: nauem. 


\section{LVIII}

Audientes signum mixaculi

Hiuc et inde concurrunt populi,

Qui per herbam sanantur singuli,

Collandantes Auctorem seculi.

\section{LLX}

Dum sic multos a lepra liberat.

Ducis aures rumor attigerat, ('uius natnm frater necanerat, Quem leprosum Redemptor fecerat.

\section{LX}

Mandat illam eomes per nuntium. () ne comicta prece rogantim Cum ministris intrat palatium, ()uam salutat turba regalium.

\section{LXI}

Ipsi quoque sedes substeruitur, Quam pro fratre sie dux alloquitur: ,Ab hac peste si per te solnitur, Nulla merces tibi negabitnr."

\section{LXII}

Que respondens hee duci suggerit: „Si leprosus quicquid commiserit Septem uiris et milhi dixerit, Ab hac lepra sauari poterit."

\section{LXIII}

Tuue rogatu ducis et ciumm Nulta dixit in aures omnimm; T'amen illud celanit nitium, Quod commisit in fratris filium.

\section{LXIV}

Tamdem gemens inter suspiri:

Et a fratre data licentia

Se peccasse dicit per omnia,

In nepotem nictus imimia.

\section{LAV}

Hoc andito comes cum aliis Expanescit ad uocem sceleris, Plus de casu dolens mulieris Quam si septem careret pueris.

\section{LXYI}

Tune matrona insuperabilis Sauans egrum respondit flebilis: "Ego illa, o comes nobilis, (F. $157 \mathrm{r}^{\circ} \mathrm{a}$ ) Que dicebar in hoc culpabilis."

\section{LXYII}

Ad hane nocem clamor exoritur, Et in laudes dolor connertitur. Laude mnlta mater attollitur, Que ut nubat sanato petitur.

\section{LITIII}

Illa carnis horrens illicita Non recepit eorum monita, Sed discedens pace gratuita Romam uenit, sed ut incognita.

\section{LXIS}

Ibi manens per auui circulum Iultum sanat in urbe populum, Duce Deo per herbe poculum Inauditum agens miraculım.

\section{LXY}

Dum sic multis impendit gratiam, Fama domm impleuit regiam, In qua miles propter fallatiam Sustinebat lepre miseriam.

\section{LXXI}

Hic est ille de quo prediximus Aceusator matrone pessimns, Claums nanis ducis et proximus, Quem premebat dolor gramissinus.

LXIl, 1: Ms. respondes. - LXTI, 3: Ms. Ego sum illa. - LXIX, 1: Ms. animiculum (?); ms. Bibl. nat., f. lat. 2333 A: anni circuIum - LXXI, 3: Hs. Clauissauus (ms. Bibl. nat., f. lat. 2333 A: (larus auis dulcis). - LXXI, 4: IIs. gratissim'; le ms. Bibl. nat., f: lat. 2333 A donne la bonne lejon. 


\section{$1 \mathrm{XX11}$}

T:undem illa denicta precibus.

(2uam dux temptaus multis sirmonihus

ynerit, utrum fratris doloribus

subneure possit his artibus.

\section{LXXIII}

Ad hee illa respondit taliter: „.ii ronfe'ssus sit miles firmiter" ('oram papa et mili pariter.

samus erit mme et perenniter."

\section{LXYIV}

'Tunc a fratre sumens licentiam Ante dueum narrat presentiam, Yuod iniuste propter luxuriam Fiatuis sui dampnanit sotiam.

\section{LAXT}

Ad hane nocem comes exterritus In inuensos projumpens gemitus Sui monstrat artorem spiritus, (F, 157 r'b) ('um tho plorat omnis exercitus.

\section{LXXYI}

Ihm sic dolor astautes macerat, Illa nirum a lepra liberat Lit post cunctis se esse reserat (fram ininste comes dampnanerat.

\section{IXXVII}

Andientes duees palatii lactnm nertunt in nocem gaudii; Quam al culmen honoris regii Renocare temptabant alii.

\section{LXXVIII}

Que contempuens oruatmm seculi ()hedire nolebat consuli, sed in aures totins populi Ita dixit liomano presuli:

\section{LXYIX}

,Sancte pater, audi qne refero! Dum hee din tormenta tolero, Votum feci, quod nulli nupsero, (nod mutare nequaquam potero.

\section{LXXY}

Adhue nestra promisit famula Se nicturam esie sub regula; Vude precor supplex et tremula: Nulla mihi dentur obstacula."

\section{LXXXI}

Videns presul eins propositum Regularem dat ei habitmm, Que carnalem consummans strepitum Deo reddit in fine spiritum.

\section{LXXXI!}

Ian nunc, fratres, stante materia

Veneremur Eius preconia,

Per yluan datur isti nietoria,

Vt et nobis donet relestia.

"Amen" dicat omnis ecclesia!

LXXIII, 4: Ms. parhempnit: - LXXX, 2: Mis. Denicturam; la correction est faite d'après le ms. Bibl. nat., $f$. fr. $2333 \mathrm{~A}$. 


\section{G}

De Nostre Dame, qui garda la femme de l'empereur de plusieurs perilx

Ms. Paris, Bibl. nat., f. fr. 1805 , fol. $90 \mathrm{r}^{0}-95 \mathrm{r}^{0}$.

(Fol. $90 \mathrm{r}^{0}$ ) [A] Romme aroit ung empereur qui aroit une femme tres devote a la Virge Marie. Si advint que l'enpereur voult aler oultre mer en Jherusalen visiter le sepulchre Nostre Seigneur, et le dist a sa lemme qui estoit tresmoble, et laquelle il aymoit selon Dien parfaitement, laqnelle lny consentit faire tout son plaisir. Et tantost l'empereur prinst congié a elle et a tous les anltres et commist a nug frere qu'il avoit a garder la dame, et s'eu partit dolent dn païs. Puis adrint que celıy qui avoit a $\left(\right.$ Fol. $\left.90 \mathrm{~V}^{0}\right)$ uarder la dame fut decen de sa beaulte et esprins oultre raison de son amour et la requist de maulvaise amour par plusiemrs foiz, mais la dame pour nulle rieus ne le vonloit. Mais pour eschiver le peril ou elle royoit. son frere, elle luy promist qu'elle luy feroit ordomner une tour en laduelle elle yroit arec luy, et feroit tout son plaisir, de quoy il fut moult lyé, et entretant fist la tour appareiller moult bien. Et quant le jour assigné a ce faire viut, la dame mist deux jeunes hommes esenier's et des damoiselles pour servir et aminstrer an frere de l'emperem ce qui luy estoit necessaire, et quant il pleut a la dame et il fut entré dedeus. elle l'enferma et s'eu retourma en sa maison. Et gourerna Romme paisiblement jusques an retour de l'empercur. Apress cinq aus la dame entendit que l'empereur venoit. Si fut moult joyense et commanda aux nobles et aux plus grans an clergié ru'ilz alassent a l'encontre du sejgueur avec elle et fist noblement adorner les rues de la cité, et mist hors sans conseil le frére de l'empereur. Qui devança la (Fol. $91 \mathrm{r}^{\circ}$ ) dame et vint le plus tost a l'emperemr. Si la diffama faulcement a l'emperenr. Car quant il luy demanda pour quoy il estoit si palle, megre et si descherelé, il respondi en plourant: „Mon treschier frere, ta femme, la maulvaise ribaude, me vouloit faire pechier avec elle et a acoustumé de faire lornicacion avec plusiens. ('ar depuis que tu partis homme n'a esté escondit de son pechié desordonné. Laquelle chose sonffirir je ne pouroie. Mais m'en vouloye departir. Si m'a jusques a maintenant enclos en une tour." Quant l'emperenr onyt annumeier si villains blasmes de la dame, il tresbucha a terre comme mort pour la grant douleur qui le feri an cuer l'espace d'une hemre. Aprés il se leva et par le conseil et confort de cenlx qui le compaingnoient il monta a cheval l'andemain. La dame avee tonte sa compangnie vint a son mari, et quant elle le vonhut embracer, la feri si durement qu'elle chent a terre comme toute confuse. Lors l'empereur appella deux de ses servans et leur commanda gu'ilz la menassent en mo parfont bois $\left(\right.$ Fol. $\left.91 \mathrm{~V}^{0}\right)$ pour la decoller. Lesquelz quant l'eurent ou bois mence, eulx disoient l'un a l'autre et s'esmerveilloient de la beaulté de la dame et disoient: „Ayons compaingnie a elle devant qu'elle soit morte. Car an monde n'a plus belle." Ainssi que eux luy faisoient violance, la dame regarda vers le ciel et a voix donloureuse c'escrioit en appellant l'aide de Dien et de la Virge Marie. Ung noble bomme aree sa compainguie, lesquelx par devocion aloient a homme visiter les corps des apostres et des aultres sains, onyrent de la dame la voix retentir par le boys, qui cuidoient que une beste fust prise et a tonte fil course saprocherent au lieu ou ilz 
avoient ouge la voix. Et quant il\% regarderent que les ribaulx vonloient villanner si noble persoune, ilz les firent tantost mowir. Aprés demanderent a la dame qui elle estoit, et comment telles choses luy estoient eschenes. Laignelle ne fist point mencion de la haultesse de sa dignité, mais humblement pria le seignemr qui l'avoit delivee upil la voulsist enmener arec luy et prendre pour sa cham(Fol, 92 roberriere suluf son honnem et chasteté. Leyuel voulentiers luy ottroya et l'enmenal avec soy et sa feumes la recent moult lonnomablement, et luy bailla ung siun enfant filz a nourrir, le quel alle nourrist plns diligentement que s'il eust ${ }^{2}$ este son fil\% at luy aprenoit a fuyr la joyeuseti dn monle et a freguenter l'eglise. Mais pourtant ne la lessa pals l'munemy a tempter, combien quil ne la peust vainere. En relle cont an laquelle ceste dame demenroit stoit ung chevalien qui desiroit l'amour de ceste dame. et par donlces parolles et promesses la requeroit de mariage. Mais la boune dame ne lny accorda pas. Pour la ynelte chose de dolent chevalier penssa en son courage comment il pourroit traiter que la damc (qui l'avoit refusé fust mise a mort. Si alvint que l'ememy le tempta tant que il rint par nuit an lit de la dame ot couppa la gorge de l'enfant que la dame gardoit, et l'enfant yüil tua ('stoit enfant de son propre frepe. Apris mist en la main de la dame, pni se dormoit, le contel de quncy il avoit l'enfant tué. Et lor's le sang de (Fol. $92 \mathrm{r}^{0}$ ) l'enfant cournt soubz le costi de la dame lir tuelle sisveilla et fut moult espoventee. Et ainssi comment elle s'eserioit lasse dolente, le pere at la mere de l'enfant s'esrijlerent tost et se leverent de leur lit "4 apporterent di la lmiere pour veoir a lenr enfant, leqne] tronverent oceis. Et si trourerent lo coustel en la main de la femme. Si fint le seigneur et toute sa famille tant douloureux que a peinne le ponrroit on dire. lit aprés le manlvais cheralier vint qui l'enfant aroit oceis, et faisoit le deul et rouloit que on condempnast a mort la dame qui n'y aroit conlpe. Et disoit a son frere: "T'u nous as amenee une ribaulde, qui pour ses meffaitz un autre terre a este jugiee a mort. Elle est digne de estre arke; la mort de ton filz le tesmoingne." Adone le noble homme et sa femme commanderent qu'on ne fist point mourir la dame et commanderent aux plus pronchains mariniess du plus prochain port qu'ilz la menassent en estrange contree. Et aimsi la bonne dame vint a la mer en tirant ses cheveulx, puis entra dedens la mer. Puis (Fol. $93 \mathrm{r}^{0}$ ) les mariniers fnrent tant esprins de sa beanlte qne il courrnoit qu'elle fist lemi roulentè, on aultrement ilz la getteroient dedens la mer. Et la dame respondit qu'elle aimmeroit plus whier y estre gettee une soy aceorder a leur desordonnee voulente. Tontes foiz il\% ne persevererent point en lenr maujvais propos, ear ilz ne la getterent point 'n la mer. mais la mirent sus mo haulte roche qui estoit on my la mer. Siur laquelle roche elle fut trois jonrs 'n grant doulenr et en pleur sans boire et sans menger, si que en la fin elle estoit si matee et si desolee que par feblesse elle s'endormy ung pen. Et lors la gloriense Virge Narie, royne de gloire, luy apparut a fare resplendissant, en disant que, „pour tant que th as sonbstennes plusinus adversite\% en soubstenant la foy de chastete en mariage, doresenavant tu sommonteras toutes temptacions. at les injures que ou t'a laites seront du tont manifestees et demoustrees clerement, ear par l'irbe qui est sm ta teste et en la vertn de Nostre seigneur tous les meseanlx a dui tu eu domneris (Fol. $93 \mathrm{y}^{n}$ ) a boire seront garis." Adone se esreilla lat dame et fut moult joyense de la vision 'pni apparue lny estoit. En disant derotes oraisons cuilly l'erbe, ne 
jamais navoit esté nue semblable, et empli ces gans. Et quant ce vint a henre de ticree, si luy apparurent mariniers enrolés par la grare de Dieu, qui se arresterent an pie de la roche et la dame les appella. Lesfuelx la virent personne de tresgrant reverence, et enrent pitie d'elle et la menerent a ung port. Et tantost an'elle fut descendue a terre, elle tronva ung mesel horrible et deffiguré, si print de l'erbe et la tribla a ung peu de vin ot luy donna a boire et presentenent fut gary. Ft de re fait fut ronoume par le pays. Empres ce vint la dame en une eité, la on estoit le manlvais of desloyal qui l'eufant avoit tui entre ses hras, lo qun

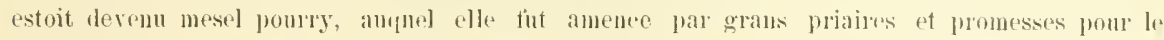
garir. Lt elle respondit que se il confessoit tout entierement ses pechiez, elle le farriroit a

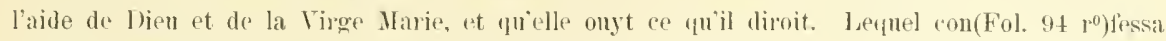
moult de pechiez. Mais il ne disoit point qu'il eust l'enfunt oceis. Adone dist la dame que tonte la medicine ne luy pourroit riens valoir on eas quil receleroit anleus pechiez mortelx. Et son frere de qui il avoit lenfant tne luy dist: "Bean frere, ourrés vostre ener et dittes tout sans rions lesser. Et se anleume chose avez fait contre moy, ju le vous parlonne." Si commenca adone a plourer devant tons les jresens, et raconta lo fait comme devant est dit. Et quant son frere l'ouyt, si plonra moult anerement, en disant du’il estoit moult deulent de la bonne dame qui asoit gardé l'enfant qu'il aroit perdn, plus que de son enfant. Arlone: dist la dame: „Sire, je suis celle qui aroit l'enfant en garde. Je ne rendray pas mal pon" mal." Et tantost elle donna a boire an dit mesel de son loebe, si fint restitné a bonne santé. Et fut moult price de demoure an païs. Mais elle respondit qu'elle iroit a Romme, pour garir phusiens meseaulx qui estojent la. Si s'en partit du paîs et ala a Romme, ou elle garit granment de meseaulx. Et (Fol. 94 V") Dien, yni est juste en tous ses faitz, a voit feru le frepe rle l'empereur qui avoit fanlcement la dane diffanee, et fint de crmelle mesellerie, tant qu'il estoit pres de la mort. Si fint la dame mandee fun nul the Romme ne conguoissoit, pour curer le frepe de lemprem, an quel dist comme a l'antre aroit fait. Et elle luy fist recongnoistre tonte la traîson que il luy avoit mise sus. et comment faulcement il l'avoit blasmoe par devers l'empereur, son frers. Et a ce furent presens l'emperew', le pape, les senateurs de Romme et tres grant partie du penple rommain. Et l'emperenr de Romme onyt le tait, comme verite estoit, si commenca a plomer et a soy latre des poings monlt sa teste. Et le pape de Romme et les senateurs et tout le penple.commença a arer et mener trestorte fiu. Et devant tous la dame garist le mesel. Et pris dist a tous generalement: "Je suis celle certaimement a qui la traisson fut faitte." Adonc eurent tous cenlx qui presens estoient monlt grant joye, et vonlt l'emperem qu'elle retoumast eneor arec loy, et tont le penple le desiroit. Mais elle respondit an pape "une aux tri(Fol. $\left.95 \mathrm{r}^{\circ}\right)$ bulacions qu'elle aroit souffertes elle avoit ronee garder chasteté en abit de religion. Adone le pape la restit en abit de religion, et resqui depuis longuement en religion on sorvioe de Tien et de la Virge Marie.

1 Ms. lempeur. 


\section{H}

De la femme d'ung ${ }^{1}$ empereur qui pour bien fere ent a souffrir moult de maulx, et estoit en grant perill de mort, laquelle Nostre Dame delivra pour ee que elle se reelama a elle

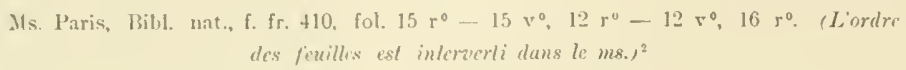

(Fol. $15 \mathrm{r}^{0}$ ) A liome ent ung empereur qui ent une moult bonne dame et sage a fomme.

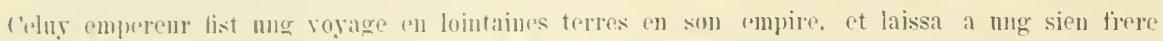

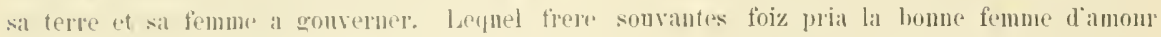
lolle et aboir sa compagnie lat sagement tous jonrs en le blasmant luy contredisoit ef clisoit que elle aymeroit myeulx que on laudist. Et celus ne cessoit tous jours de liamounester. Et advint que une foys olle luy dist quilz alassent en une forte tour qui estoit en l'oustel. at ne fe disoit pas prour mul mal, come rous onyres. Et it cuidoit que ce fust pour peehe et pour abiter a clle. (quant ily furent dedaus la tomr, ot la dame sault hors et ferma la porte, ne despuis ne le laissa yssir hors juyues l'emperemr, son mary, fut renu. Et tousjours (Fol. 15 ro) pour le resjonir la dame luy donnoit du pain et de lean par me fenestre qui estoit en l'uys, -t puis le laissa alle pour ce quil lny promist que jamais ne la prieroit. Et luy requist qu'elle ne l'accusast devant son frere. Il monta a cheral et s'en ala auderant de son frere l'emperenr monlt maigre ct atout la babe grant et les chevenlx, qui hy estoient crenz en la prison. Limpereur luy demanda ponrunoy il estoit si maigres et avoit si grant barbe et si grans cherenz, si luy dist: "Certes. sire, ung petit apure ce que rons fustes partis rostre femme me pria plusienurs foiz de pecher a elle, et quant je vis quelle ne me cessoit de prier. je me mis en la tour an pain et a l'eau. affin que l'ennemy ne me temptast de pecher, et me sont en la tom les cheveuz ainsi crenz come rous royez." Quant l'emperenr fint a denx journeez de liomme, sa femme ala anderant de luy monlt homnorablemant soy penssant que il la rist moult rolentiers. Et sitost qu'elle fut vers lny, il la ferit monlt vilainement th pié et la getta par terre, et commanda a quatre de ses escryers que tome manlraise putain la menassent eu ung hogs ot la la laissassent et loceissent de lems espees. Les escuyers la menarent, et come ilz virent quelle estoit si belle dame, ilz roulirent aroil sa compagnie, avant quilz la tuassent. let alle ne s'i.roult consentir pour riens, mes forment se deffendoit en enlx disant que elle amoyt myeulx qu'ilz la despessiksent en pures piecess qu"elle fejst ung tel peché. De la volenté Nostre seigneur il passoit pour la mog grant seigneur. qui loụt et leur osta et la mena en son hostel. Le sire et la femme virent pu'elle portoit visaige de bonne ct vauliant femme, la tindrent honuestement all leur hostel. et alle se tenoit moult devotement et nomrissoit et mettoit couchel avere ello mog petit entlint du seigneur ct de la tame. Le sire aroit ung frere gui celle boune

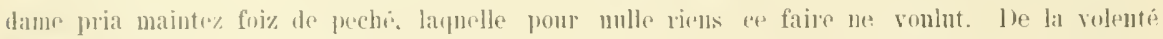

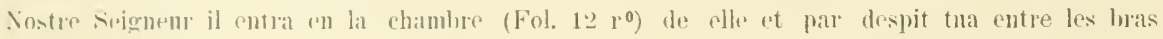

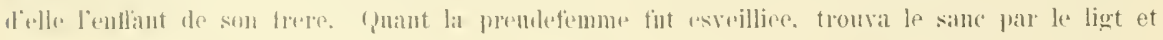


l'entfant mort, et eria si fort que le sire nt la dame et tons cenlx de l'ostel l'onyrent, et tons s'i assemblerent entour l'euffant. Et ilz menerent grant torment. Coluy lirere du seignenr qui aroit tne l'enffint l'enst tuee, qui nt la luy eust ostee. Et. disoit a son frere que on la tuast. aiussi come elle avoit tue l'entfant. Lit fut juger de plusieurs a morir de diverses mors. Le pere et la mere, qui l'avoient rene monr bome vie, ne crment pas qulelle l'enst occis, of ne crument pas reulx qui ba jugoient. mez sercement la mirent en ung batean dedaus la mer, et dirent: „Si elle a fait le fatit, elle monra en la mor, et celle ne l'a tiait. Nostre ségnenr la pourvoya." Et ainsi sens nul vive fut mise en la mer. Des mariniers la trouverent d'aventure ef la virent moult belle dame ot voulurent aroir sa compagnie. Elle pour riens ny accourdast. mez vouloit myenlx morir tonte semle en la mer, on que on la noyast. IIz la laissarent tonte seulle en me ysle de mer, et la elle pria moult devotemant la henoite Vierge Marie, qui luy roulsist ajder ot la secourir, aiusi come besoing lny estoit. Et en icelluy mechiel s'endormit. La Vierge Marie vint a elle, et se esvella, et luy dist: "Prens l'erle qui est soubz ta teste, car elle garist de toutes meselleries." Jit en l'eure la print et vit nne nef elargee de religienses persomes et derotes qui pour l'aris de lien la viment querir et avereques end l'en menerent rot la mirent en terre. Elle vist ung meseau et pom l'amom de Dien le gurrit, et aprés celuy mains aultres vindrent a elle qu'elle guerit. Le sire de celle terre estoit celuy a yni l'enffant avoit este thé ou ligt entre les bras de elle. Et la manda a rnerir, affin qu'clle gnerist son liere (qui estoit devenu mesean, si vint et le cognent, mez ilz ne la cognoissoicut (Fol. $12 \mathrm{r}^{0}$ ) pas, ear elle estoit monlt muee des grans doulems qu'elle avoit ens, ear alle avoit en moult de grant fain, de froit et de ponvreté, et anssi sa robe avoit vendue pour avoir du pain. Si luy va prier le seignemr qu'elle voulsist guerir son frere. Elle luy respondit qu'elle ne pouroit, s'il ne confessoit verité, car la maladie luy estoit vemue pour une grant mauvestié qu'il avoit fete Adont il dist a son frere qu'il luy pardomast et qu'elle disoit verité. Et son fiere luy pardoma. Adont luy confessa commant il avoit tue son enffant en despit de la preudefemme, qui ne se vouloit accorder a luy. Adont la prendefemme le gnerist et se fist cognoistre an sejgneur, et en enrent monlt grant joye, ct demora arrieres leans come derant. Le frere de l'empereur par sou peché devint mesean et onyt d'une boune prendefemme qui garissoit de mezelerie. L'emperenr, qui estoit son mari, l'envoya querin' Quant elle fut venue vers l'emperem, il la pria desant le pape of plusiems de ses cardinaulx, disant qu'elle voulsist guerir son frere, celuy pour qui la dame fut ferne dn pié. qui pas ne la cognoissoient. Adone elle respondit devant tous et dist que celle maladie lny estoit venue pour nug grant peché qu'il avoit fait, et qu’il n'en pouvoit estre guery, se il ne le disoit devant tous. Adoncues le mesel dist qu'elle disoit roir et cria merey a l'empereur, son frere, et lui pria qu'il luy voulsist pardonner, et il le diroit. L'emperen, desirant sa guerison, luy pardomna. Adone il ra dire commant il avoit prié do peché sa femme et commant pour riens elle ne s'i volut aecorder, et dist tonte la verité dn fait. Quant l'emperenr l'onyt, il hent si graut denll que a merveilles, et erioit et se complegnoit monlt fort de ce que si preudefemme et si devote avoit esté ferme et perdue, et jura qu'il ne resseroit d'aller tant que en sceust certaines nonvelles. La dame, qui monlt tormenté le rist. ( $\left.\mathrm{F}^{\prime} 0 \mathrm{l} 16 \mathrm{r}^{\circ}\right)$ a grant poine se fist acognoistre a luy et luy dist tontes les aventures qui luy estoient avennes. Adone l'emperenr et tous cendx qui pres estoient furent mont jojeulx. Et aprés tantost guerit le frere de l'empereur. Et par ainsi la 


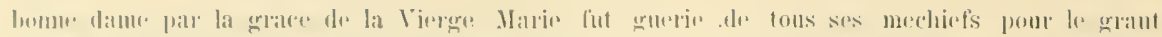

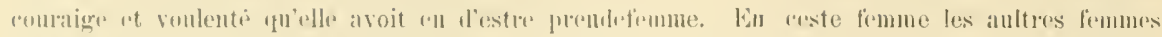

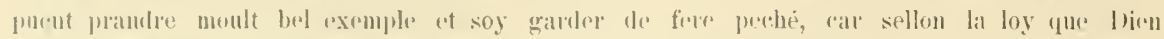

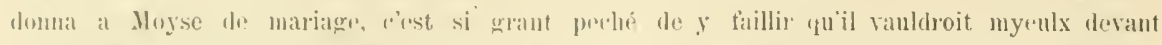
morir. Lit pour lus ortlonua bieu que tous ceulx et tontrs colles qui ce feroient "fui fussint litpides, come ancore en plusieurs reaulunes ere lait r.t en plusienrs ysles de mer.

\section{I}

De la sainte empereris qui garissoit les lieprous

$$
\text { Ms. Paris, Ars. } 3516 \text {, fol. } 133 \mathrm{r}^{\circ} \mathrm{a} \quad 136 \mathrm{r}^{\circ} \mathrm{a} \text {. }
$$

(F. $\left.133 r^{0} a\right)$

l)ames, "fli vostre mariagu

T'enés en loi de putcelage.

(pui netes ros tenis al momli.

Et hatelliés si rostre esponde

5 Qu il n $\mathrm{j}$ pase mule pensee

(ui mout ne soit de bien armee.

Entendes chi et por ilprendre

Quant cil aprent qui set entendre!

D'une dame vos roel conter'

10 Qui mout bien sot son cors garder.

(ïr porés ancui ol coute

Que pren i a. pui bien le donte.

[hanes, por linell, cutendes i!

Nonmeement a rons lo di,

15 Car ne saves mie tresbien

f'oum'ut on doit sor' tote rien

Dariage easte tenir.

J'en ai veï sovent venir

Mainte cruël mesaventure

${ }_{2 n}$ Dont pecoüe est la jointure.

('i) mesfait mont qui le peçoie,

('ar, s'aupues lasque la coroie,

(In le doit bien si fort restrandre

Que glons morsiaus n'i pnist ataindre.

25 Vous, puceles, rous, mariëes,

Garder devés si vos contrees

Et ros eastians por bien manoir (que nus asans ni puist valoil.

Se vos unl bien voles aprendre,

30 A cest conte devés entendre,

f'ir d'une dame est la matere

Qui cneaï en grant misere

Et sofiri mainte pestilenst'

Por bien garder sa consiënse.

3. Mout garla bien sa loianté,

Et s'ot elle mout grant plente

bentecement por li decoivie.

Mais ne volt onques mul jor boive

De la puison we Iel bevage

41) (ui pecoiast son mariage.

Li lons prologes pas n'aunit, ('ar jou n'aroie dit a unit Con graus preas est d'extre loial.

I hames, vous yui prosés le mal

t5 Lt contre Dien voless ovrer, Venes vous faire relever, Terès aprendre c'on doit faire, ('al cis contes ros doit mont plaire!

(F.133 $\mathrm{r}^{\circ}$ b) Escoutelle tant solement

5i) Et puis, se vos avés talent

De bien faire. si l'enbriciès,

Et se çou non, si le laisiẻs!

A Rone ot un enpereor.

Rice d'aroir, poisant d'onor.

25. Ms. maries. - 36. Ms malt. - 38. Ms. boire. 
5. Joius justicins estoit of piu. (om'il avient en si haut liu, Religiëus de boine foi.

Aine puis n'i ot nul mellor roi: En Lien avoit tote s'entente.

bo Mollier a prise bella et gente

Et hantement enparentee

De tot le miols de la coutree, hivene et oneste et liele et caste, (Qni sa jornee pas ne gaste.

65 lin Dieu servir est ses manoirs;

boins eïrés ert li manoirs

U tes dame maint et converse

Qni le pensee a si diverse.

Aubedni ont un essiëut;

70 Et sa dete si bel li rent

Li enperere sans folie

(2u'il n'i a point de vilonie

Mont s'ainment et se sont amé,

Si en ont si le trop ostẻ

75 ( ine tot lor estre et lor maniere

Nesnrent bel et a rastiere.

Del conble sont adés estrange;

Por cou lo font ane il nespange.

En lor afaire sont moirm.

sn si bel s"i tienent et si bien

Que lor sotes et lor enprmut

Si justement et a point sont

Qne en nul Jin ne se descordent,

Car lor manieres s'entracordent.

${ }_{5}$ Joians putet cotre et lie Rome,

(Qui a tel dame et si fait home

Lit si le tient a grant honor

Con sont cangié puis li sengor!

Tot le païs tint si en pais

9o Ne li covint de son palais

Hovoir por esmore de gerre

('ar en pais ert tote la tere.

En lui n'avoit point de desroi;

De grant honor; de bel conroi

95 Estoit li sire en tons endrois.

Preudon estoit et mont cortois,
N'avoit orguel ne nul desdaing;

Enperre ert et boins compraing

Lstoit as gens et gentitis sire.

1no Qui de la dame vos violt dire.

Mout se set loiamment tenir.

bieu le porés aviut oür

L'enpereres vit son pais

Tot plain de pais, tot plain l'anis tos Por loien plus dingnement proier

Tolt il sains lius (yncre et errkim Qui sont espars par la rontree.

Piec'a yntil i ot sa pensee,

Or le vot il a vevre metre.

111 Ensi con chi nos dist la letre,

Il prist avoir et compangons

Et escuiers et boins garçous

Qui corenant sont a tel voie.

Mais li depars n'est pas a joie

115 De lui et de l'enpereïs,

Car il i ot grant ploreis

Por cou que l'uns l'autre Iaisoit.

A grant paine se departoit

De sa feme li enperere.

120 Un jovenencel avoit a frere,

(fue mont amoit, si l'apela;

L'enpereîs li romanda:

Mlout li pria cyue por s'amor

Face a son fiere tant d'onor

125 ('ue gré l'en sace al repairier.

A tant s'en ront entrobaisier,

A grant anui se departoient.

Les gens qui environ estoient

Plorerent mont de piëté

130 Por ens et por lor amisté.

leparti sont a ruel (fu il paine.

rele remest, qui mont est plaine

De grant dolor et de grant ire.

Al departir plora li sire,

135 Et la dame est en mi la voie,

(Yui sou singor des iols couvoie,

Tant que le pot des iols reoir.

Adont Je convint aseoir 
Par mont grant torece dlo solor.

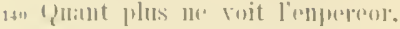

Ein son palais sen retorma.

lill sa camble antre. mont plora.

Mais il ne th ains mesurstancer

yui no traisist an oubliane"

11:i Lal damer fil de huin eosutinet.

ii eslut linen lo droit red tort

lit si sot liem mreler justice.

Lal deritare de saint' Figlise

F. 13: rod Maintint lian et rasisablement.

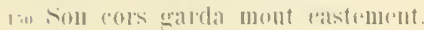

lin tot l'afaire $\|$ rlle fil

Aroit ener domme adex restu

lit pald les allmes de siälas.

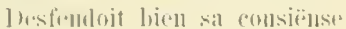

1.5.) Ih mut of bien avirenee

Lientrmelement de sat priscer.

sinpee tint tot adlis tritite,

si $k$ '⿺乚 sen mur u’ot mule liait.

le jovenencel fiat grant honol

16 in (1)i freles elt a son singor;

Mout jue a lui at mout li rit,

Mais ni entent antre delit

fors con yucle onorer le riols.

1h son singor forment se tiolt,

16.. $5 \mathrm{~s}$ "n portrait la ramenhrances

En son frere por lonorauce.

Elle l'aime por onorer.

Mais dantre amor lo violt amor

li jorenencians, qui le lemir

170 ]'or cou yu'i] doute l'escondire

Nose l'amor pas dexeovrir.

Et neynedent li fait seutir

Amors si cruelunent sun dart

?ue il de li ne se depart.

17.5 le li departir ne se puet:

Voelle u non, amel li esturt.

sircies mout a dolente vir,

pront il i part sums la partie
(Mil covoite plus a avoir.

lan Imors le set bien deceroir.

(yui tel partie li lepart

lont il ne puet elamer sil part.

('bascun jor croist et rien ne monte;

chou fait Amoss. tui lo mescouts"

ans Tot lo loier de sa jurmo.

limporrous est mout amrow

the lold amor; rers la lolio

Fistrive mout sa caste vic.

be cer ne set mot linjerere.

1.t1 Ji jovenencians, qui est ses lorre

Fit ses mainsues et ses noris,

Mrut en l'anor lempreis

le nom de la fraternite.

s'amols, qui l'a si enbrase

19.5 kt xi espris, li fait seutir

fhoul dont il violt vers li mentir.

llesfait il dont, se il en ment?

Amors, par rostre jugement

(F $\left.133 \nabla^{\circ} a\right) \quad \mathrm{N}$ (mesfait riens qui ment isi;

2no $1 l$ ais il fausnic son ami,

Ne fait pas bien par droit eseart.

Amors li fait sentir le lart

Par coi tel chose a enbracio

Dont la dame ert mont arillie.

$2 n 5$ Amors li tot bojre et mangier

Fit se li fait par nuit vellier.

Et a cele eure quil soumrile

si le semont. et il s'esvelle.

Tant yüil ne puet renir a songe;

2ar Et roirs li sanble la mengonge:

II peuse çou k'estrt ne doit.

Sa crraus folie le deçoit,

bont la thanbe est adés coverte:

Fait som penser aler a perte;

21.; Et, quant il ne pot plus sofrir,

si li coviut adés corrir

F.t son pensé et sou afaire.

Ii cors, qui a vestu le laaire. 
Le vient destraindre de parler,

$220 \mathrm{Si}$ ne s'en pret or mais celer.

Morir l'estuet u descovrir,

Iais mont grans foree est de morir.

Por le morir qu'il a si prest

S'en rint a perdre son conquest.

22.5 La grans amors qu'il a l'ocit;

Por l'ardure qu'il a l'a dit.

A la roüne en est remus.

Pliss de mil fois li est ceils

Li hais del dire et li voloirs,

2.s0 Mais tole amors ot ses pooirs

L'enbrace si fort, tol l'atire

Qu’il ne s'en pot plus esemdire.

A li s'en vint, merehi li erie,

Hais ne l'ose nomer amie,

21.5 Ains dist: "Dame, merehi vos proi!

- Amis, fait elle, et ros de coi?

- De coi, dame? Je vos aine si

Qu'il me covient criër merchi

Je ros ane, dame! Cull m'amés!"

2+n Dist li dame: "ravoir pöés

Que je ros aiuc por vostre frere

- Dame, eertes li enperere

Ne ros reeuist pas de tel cose.

- De coi donques? - Dire ne lose.

245 - Osés le moi, si le me dites!

Se c'est mesfais, s'ell soiés cuites!“

,Nest pas mestais, se jon ros aim.

Se vostre amor roel et reclaim,

(F. $\left.133 \mathrm{v}^{\circ} \mathrm{b}\right)$ Nus ne se doit de çou doloir.

250 - Amis, bien croi que dites voil,

Se ros tele amor me querés

Dont li miens cor's soit onerés.

- Honor, dame, tresgrant ros porte

()ui tel mesage ros ajorte

25s ('omme d'amor boine, enterine,

Dont endroit moi avés saisine.

- coment amés en tel matro?

- Montplus por moi que pormon lierer.
- Amis, por lni certaimement

260 Vos aiı jou bien et loiaument.

La loiantés n'est pas oseure;

s'amol i a, e'est par mesure.

Et, se mesure en vos trespase,

I ont est lamors endroit moi hase.

26. Ne me roel pas tant abaisier

Que mon singor voella laisier.

("or ei a mout dolerens ange,

he de monor le fac estrange.

- Cangier, dame, je ne roel mie.

270 l'or con, se derenés m'amie,

Que vors l'enpereor cangiés

Et moi et lui acompangiés,

Tostre anor's soit en nos dens mains.

Miens soit li plus et siens li mains.

275 - foment poroit çou arenil'

Que j'eusi puise departir

M'amor que j'ai a lui donee?

('hi aroit fole deserree,

('iu' sos del nom de donce amie

zsu Ne pnet nos faire deprartie."

„J'aim mon singor et ros por lui.

Me proia il de mon anni

Que ros amase et vos joïse?

Por rostre amor pas nel fesise,

zжs Se por lui non tant solement.

('ar esgnardés droit jugement

sod lautre amol yos doi amer

Fors solement de l'onorer;

f'ar de cou me pria vos frere,

${ }_{290}$ Ne me pria pas lemperere

Que ros amase d'antre amor,

Et, s'i pensés nul antre tor,

("est folie, si con moi sanble.

(pui folement a rous s'asanble.

29.; ("est en presure de folie.

Nel portés longhe conpangie,

Mais laisiés le, se bojn ros est,

Car je n’i roi point de compuest 
F. $\left.133 \nabla^{\circ} \mathrm{c}\right) \quad$ ('ar, se vos m'aviés oi romquise

зиo Et a rostro oés desos rous misr,

ïn series vous las et peciere.

Nais jo n'i roi nule maniere

Que par raison i puise eslire

Fors solement de l'escondiro."

sus ('il dist: „J)ame, al mort sui forus,

s゙a l'escondir' sui renus.

Dame, por lojen, nel penses ja:

('ar, se jou muir, par vous sera

Loeoisons estes de ma vie

suo Et de ma mort, suer, doce amio!

Amie? Trens! jun ai menti,

Mais ensement le cousenti

Mes ruels, qui est fins et loians:

Me fist de rest mot estre ismians.

315 Loiaus est il, u voelle $n$ non:

bil noi ne truis mule oroison

Fors de lien amer loiaument.

Jlais ma compange me desment

(uii me deüst acoupangier,

320 loont si portise de lerier

Le fais une $j$ al tos sens rmpris."

Jist la roüne: ,Or m'est avis

(pue tu maimes outre mesure.

the poise moj que la rlroitme

32.5 As pasee l'anor certaine.

C'este seroit fole et vilaiue,

Te je n'ai soing d'amor (mp)rendre

Dont ma corone puist desrendre."

lsi se plant cil rhascun jor,

sз (pui sopris ert de tele anror:

lal roïne est icl tot soprise.

(puant la parolle est ensi prise

Que cil le violt amer isi.

"Un jor, fait elle, mar vos vi.

335 Que conmandés me fin cis firere

Et que mesire l'emperere

Parti de moi, lase, raitive!

()r ne sai comment doir vive.
Ma caster rorai garder; atn. Ja nus ne s'ell pora jabur

("ou folie truist en mon cors.

Ançois me puist prendre la mors!

Ancun consoil m'esterra prendre

f'omment me puise an fin desfemlre

at.i f"st reritès qu"il en palist

Ft mout enpire et amagrist.

('ar il ne looit ne ne mangue.

Tos li corages me remue,

(F. $133 v^{\circ}$ ) Quant jou le roi. Mout me pesast

as̆ De son mescief, s’il ne m'amast.

Ne m'aime pas, ançois me let!

Ilout par est fols. se if ne set

() ne por proier ne por avoir

Se poroit pas mamor avoir, 3.. Xo jou nel puis loughes sofrir.

la riens el mont que plus desir.

("est con que mesires venist,

(pue de tot cou pais me sesist."

'T'ant a l'anpereïs pensé

s60 (ju'ele a mont boin consel trote

l)e eelui metre ('ll sus de li

()nyues a home ne jehi

le sa pensee nule rien;

A tote gent le cola bien.

365 Maçons manda par tote terre,

llont entretint, mont en fist querre

Por me tor edefiver

Dont elle dist yn'ele a mestier;

Nont cnevre bien tot son afaire.

370 La tor fist grant et liaute faire

Et par dedens bele et pavee.

si $k$ 'a degrés en fu l'entree.

Quant la tors fin faite si bele,

Li jovencmeiaus, qui renovelle

375 A la rlame s'anor premiere,

La roinc cuiert del maniere

() ise il ne muire ensi por li,

('ar il se sent del tot failli. 
Liamors, qui est vers lui si hante,

${ }_{3 \times 2}$ Le met en poine et en defaute;

Et la dame li aconta

Que son plaisir del tot fera,

Mais elle a el en sa pensee,

Car n'ell roroit estre blasmec

385 Por trestot l'or qui est el mont.

li jorenenciaus l'ot et respont

Mout humlement: „Wame, merchi!

Tos sui garis, s'il est ensi."

„Ensi est il, bians dous amis;

390 ('ar jou te voi si fort sopris

N'en puis sofrir la mesestance

Que tu n'en aies alegance.

Bians es. Merchi en doi aroir,

Et je ferai tot ton voloir

39 Et loiaument et rolentiers.

Je te siurai, va t'ent premiers

En celle tor fresce et novelle,

Qne por t'amor ai fait si bele.

(F.134 ra) Cos la dame ensi parler, $400 \mathrm{si}$ se coumence a conforter.

Al cuer of si tresgrant contort

f'on s'il resusitast de mort.

('il monte avant, cele' le siut,

Qui en siunt bel le deçut.

405 A l'nis vinrent, cil i entra,

('elle lenclost bien et f'erma,

Qui par defors s'estoit forselose:

Car n’i entrast por nule chose.

Dens damoiselles i tramist

${ }_{410}$ Et deus sergans entrer i fist

Qui le baceler serviront

Et soll mangier la sus trairont,

Car par mi l'uis mus n'entrela.

L'enpereïs en portera

41.5 Les cles; n’i violt croire nului,

Car mont redonte son anni.

Ensi est cil enprisones;

Mais d'une rien s'est porpenses
Que, se ja mais rient l'enperere.

420 Qu'il trovera cruël matere

Par coi l'eupereis ert morte.

Iceste riens le reconforte,

Car mul confort n'a d'antre part.

Amors l'seprent, le cuer li art,

425 Ilais il ne set or nnle roie.

Par coi ses cuers revienge, a joie.

La roüne est en son palais,

Et li Rommain furent en pais,

Car el paîs n'avoit nul home

43، Qui dont peïst geroier Rome.

Cine an estojent aconpli

Qne l'mperere s'en parti,

Mais or s'est mis al repairier.

Avant vinrent li mesagier,

435 Qni ceste novelle ont noneie.

Lemperreïs se fait mont lie;

Ses grans ators et ses baruages

Demostre bien que ses corages

Ert mont haitiés de sa renue.

4t0 Trestote Rome est esueïe

Par le conmant l'enpereïs.

Es rous a tant de ses nouris

Que l'enperere i envoia

Qne l'endemain matin venra.

* ${ }^{\prime}$ Par la cité mainent grant bruit,

Ni ot gaires dormi la unit;

Les gens i ont engordinees

Les rues et bien acesmees.

F. $134 \mathrm{r}^{\circ} \mathrm{b}$ ) L'endemain en chascune rne

tso Ot grant joie por sa venue.

'T'uit s'aparellent par la rile,

Li serf, li frane et li nobile;

A grant feste et a grant ator

s'en vont encontre lor sengor.

$\$ 55$ L'enperreïs nist fors son frere,

Qnant elle sot que l'enperers

Devoit renir, mais mar le fist,

Car puissedi s'en repentist. 
Selle peüst, mais ensi fu

two (que malement est avenu.

Cil issi for: magres et las;

11 i paroit qu'il n'eüst pas

Füb bien sa volente.

Aroec les clers de lia cité

465 Et ensanble la noble gent

s゙en issi fors isnellement.

lienperrers, qui ert montee

Mout ricement, fu adestree;

Dehor: s'en ist a grant conpange.

tio Et li varles, qui pas nadange

Ne son aler ne son venir,

As premerains s'tn vait isir.

Que tot arant vint a son frere.

Et. quant le rit li enperere

475 Si las, si magre, si desfait,

lemanla lıi qui çou a fait.

(iil respondi tot en plorant:

"Sire, dist il, jou vieg avant:

Conter ros roel une norelle

tso Qui ains nul jor ne me fu bele.

Vostre feme, la rius, la fole.

Dont tos li mons crie et parolle,

Fos a honi et s'est honje.

Sa manvaisties, sa puterie

t*5 Et ses outrages a tant fait

Que nus n'en riolt aroir n'en ait.

Tote est renans, tote est conume.

Et ros andies quiele fust une

A vos tot senl; mais antrement

t9o A reploitie a mont de gent,

Si le set bien et cil et cele.

A moi meismes se rolt elle

Aconpangier de folt amor,

Mais jon me mis en une tor:

495 P'or soll que jou nel roc soufrir,

Ne mosai faire plus veîr.

Por cou casti ta dingnité,

Ta sengourie et ta bonté
(F. $\left.134 \mathrm{r}^{0} \mathrm{O}\right)$ lit quier, por Dien, ja mais ta bonce suo A lo soje a nul jor n'atonce,

C'ar trop seroics rergondés,

Se mais estoies ses privés.

Honte t'a fait grant et aperte,

s"en doit wes cors aler a perte,

son Car de toi s'est desmariëe.

Honie soit et rergongdee,

Car mesfait ta certainnement."

Lienperere, quant cou entent,

Fu mont iriés, si n'en pot mais,

${ }_{510}$ Car il cuidoit k'en nul palais

Ne conversast ainc mioldre dame.

Mont s'esbahist de ceste fame,

Mout fu dolans, mont l'en pesa,

si s'est pasmés del doel qu'il a.

515 Quant il revint de pamisons,

S'entendi auques de raisons.

Ses persones le conforterent.

Qui sa grant ire li blasmerent.

Lienperere s'esmerrella,

520 En son coraje s'apensa

('or estoit il trop mal baillis:

Deceï l'a l'enpereïs,

U il enidoit trestant de bien.

Perdu en a sor tote rien

$325 \mathrm{Et}$ son cnidier et sesperance.

Or n'i a mais nule fiance,

Car on l'en a tot desfiẻ

Desloiamment par fauseté.

Lienpereres, qui mot n'en set,

530 Lenperrejs a grant tort het;

Decenis est de la haïne.

A icel point, a cel termine

S'en rint la dime par amor

A l'encontre de son simgor.

535 Quant elle i vint, sel rot baisier

Et acoler et enbracies,

L'enpereres l'a si ferue

Que clle est a terre ceüe. 
Lemperreïs fu mout dolente,

540 Et çon li torble anques s'entente

Qu'ele $n$ i a niënt mesfait.

Encontre force n'a mul plait;

('ar l'enperere a ipelé

Dens siens sergans et commandé

545 Quil le prongent isnelement:

Par son esgart sans jugement

Le roisent decoler a paine

En lit forest ilnec proçaine.

(F.134 $\mathrm{r}^{\circ} \mathrm{d}$ ) Les gens qui sont ilnee entor

550 Noserent pas l'enpereor

Merchi priier tle cel forfait.

Leenerere grant tort li fait,

(ui le laidenge sans raison;

Et cil a fait grant mesproison

555 Qui sans forfait isi lacuse;

Et li jugieres qui refuse

Son escondit ne fait pas bien;

Andui mesfont sor tote rien,

Li jugiere et li encusere.

s6" De çou ne set mot l'enperere,

S'en doit estre mont mains blasmés;

Mais nequedent c'est verités

Il deïst ains la cose aprendre

Qne tel rengance en deiist prendre.

565 La dame est as sergans livree,

Et la gentis et l'oneree

s"en vait morir a deshonor

Por le fans dit d'un traïtor.

Eu la forest ensanble entrerent,

570 Et sa bianté mont regarderent

Li dui serf, uni l'ont couvoitie.

Isi com'elle estoit irie,

L'ont aherse de totes pars.

Or en soit Dens li trais regars,

575 Qu'il le detraient a grant houte:

„E! boine dane, or ros mesconte

Honeurs trop desraisnallement,

Et lone de vous sont vo parent.
Ja mais, dame, n'arės jor joie, 580 Se Dameldieus ne ros envoie

Proşain secors par sa pitié,

Car çou n'est pas par ro peciè

Que vos arés ceste arenture,

Qui si ros est et pesme et dure."

${ }_{585} \mathrm{Li}$ dni sergant l'ont mont detraite.

Ja li eüsent force faite,

Quaut uns frans hom oil les cris

Que fist la lase enpereis.

Pelerins ert, ilnec aloit,

590 Par la forest alont pasoit,

Car li cemins i ert roiaus.

Li chevaliers ert mont loians;

Les eris oï par le boseage,

Cuida che fust beste sauvage;

595 Cele part ceurt il et sa gent

A esporons isnelement.

La roïne a ilnec trovee,

Que cil aroient detiree,

F.134 $\mathrm{v}^{\circ} \mathrm{a}$ Qui mout grant force aroit rendue,

600 Et si l'aroient ja rencue.

Li pelerin la dame virent

Et sa color sovent remirent.

Por sa color, qui tant est bele,

Qui mellee est en la maiselle,

605 Ociënt rels qui le tenoient

Et qui la dame detiroient.

Li sire l'a bel aparlee,

Si l'a encuise et demandee

La mesproison de soln alaire.

610 Celle li ment tot el retraire,

Qu'il ne l'en soit mesarenu.

Dist l'ms a l'autre con mar fu

si bele dame et si tresgente,

Car, s'elle avoit anques de rente,

${ }_{615} \mathrm{Si}$ seroit elle bien asise.

Li sires a la dame prise,

Monter la fait. Cele li prie

Conme dolante et esmarie 
(yual ait por liben merehi de li, 62" s"il de dame of onques mereli.

lij gentis hom mout l'aseiire

El s'en fait prendre ment grant cure.

En sen pais la fait porter,

U il la fait mont honores.

625 sia feme estoit de grant noblecre,

si le maintint a grant ricere;

Ilout le goï et mont lama

Et un sien fil li conmanda:

N"en aroit plus: forment l'amoit

630 La mere et mout le errisoit.

L'enpereïs l"a receii.

si l'a mont longement eii:

Iout l'onora, bien le nori.

Tameldien prois ades merchi

635 Qun'il li ait, et il si fist,

Isi ennme li contes dist.

Bele dame ert et nete at sage

Et si aroit humle corage,

Ne riens u'aroit desos sa guinple

an Qui ne fust boine chose et sinple.

Dedens son cher n'avoit nul cange,

De tot lo siecle estoit estrange:

Si belement s'en estranga

C'onfunes nul jor ne se canga;

o.5 Dameldien aime et Sainte Eglise.

Mout fort l'onemre et mout le prise

La dame; autresi fait li sire.

Nus ne ros purt conter ne dire

Son bon estre ne sa vaillance,

(F.134 $\left.\nabla^{\circ} b\right) 6.50$ La ralor de sa contenanee.

Mais li diahles renimeus,

(Qui mout est lel et curjëıs.

$\mathrm{Li}$ fait asant liver et rendre.

Car son castel ruide bien prendre;

65. Mais castees l'a si garui,

Qui antre fois le desfemli,

liencor lo cuide bien tenir.

Mliols riolt l'enperreis morir
K'envers son cors onques mesface 6io l'or nule rhose con li face.

Mout le destraint et mout l'asaut, Mais sacjès bien petit li rant:

Il se puet bien vers li coubatre, Mais niëns sera de l'albatre.

66.5 En la cort ot un cheralier,

['or cai elle ot grant enconbrier, Qui mout l'ana por sal biaute,

Mais il ne pot par verite

Ne por menconge de pronetre

wo Dedens s'amor enbatre et metre.

II l'aime plus asés que doit,

Gue li diables le decoit;

Mout le blandist, mout le losenge,

Mais cele met par tot calenge.

${ }_{675} \mathrm{Or}$ li descherre son corage

havoir le violt en mariage.

„Mariage! fait elle, sire,

l)e çon ros doi bien escondire,

Car freres estes mon singor,

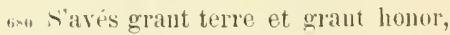

Et je nai nule chose a prenclre.

A moi ne doit tes hom apendre,

Ne jou ne pens pas tol folie

("a moi afrist tel sengomrie, 6s.5 si sai tresbien que me gabis.

- Avoi! dame, fait il, sofrès

Je ros en ferai tel fiance

Que sauve en ert la convenanee.

- Biaus dons amis. gon ni a mie. 690) ('ar jou n'ai eure d'estre anic,

Ne ja amie ne serai.

Laisies me en pais, je le dirai

A vostre frere u a ma dause.

Ceste proiere mout m'adame,

695 si n'en roel mais oür parler."

(il le laisa del tot ester,

Mais il porpense de sa part

Par ques engiens et par quel art 
II le pora traire a lat mort. $\left(\mathrm{F} .134 \mathrm{v}^{\mathrm{C}} \mathrm{c}\right)$ too Traïr le violt a mont grant tort,

(ar al cuer a mout grant pesance

De çon qu'elle l'ot en viltance

Et de gou k'amer ne le violt.

IIont sesmerrelle, mout se diolt, 705 Si a trovee la maniere

Par coi la lame ert mise ariere.

Par nuit, isi com'il se gist,

Est sus levés, un cotel prist:

Ij coutians ert graus et agus; $710 \mathrm{Al}$ lit la dame en est vemus.

L'enperreïs, чni se dormoit,

Entre ses bras l'enfant trinoit,

Qu'ele amoit mont de grant anor:

Niess ert celui, fis son singor.

71. Li chevaliers, „ui arant vint,

Lagu cotel en sa main tint,

L'enfant trenca par mi le cors,

Si que li sams en sali fors.

La main la dame suët prist

720) Et le cotel detlens li mist,

Puis sen revint concier ariere;

L'enfant ocist en tel maniere.

hayns ert mors, mais or revit

En çou que jou ros ai chi tlit.

${ }_{725}$ Cis est plus fel de lui aspes,

Car cis Caüns n'ert pas lasés

De faire mal ne de penser:

llont a le cuer dur et amer,

Quant il cestri ocit et tue.

7зо Or est la dame deceine,

Qui ne set mot de cest afaire.

Alyy! traïtres deputaire,

Com'a chi dure traîson!

Oejs arés a desraison

73.5 Et murdri l'enfant rostre frere,

Dont traitre estes et mordrere!

sor la dame li sans espant;

Quant elle sent sanglent l'enfant
Qu'ele tenoit. si s'escria, ito si que li sires sesvella.

surs est leves ismelement,

Et celle plenre tenrement.

Quant la dame fu esvellie,

Mont fiu dolante et mont irie,

its Quant son enlant roit ensi mort

Lase! hi n'a nul hoin confort.

li fus lu mont tost alumés,

Et li conteaus, yni fu trovés

Et unu'ele tint es sa main destre,

(F $\left.131 v^{0} d\right)$ :50 Lor fait saubler que roirs pot estre

(Qne l'enfant ait por voir ocis

Li sire en est mont entrepris,

('ar il ne set qu'il en prist dire.

C'elle est preste de l'escondire,

7hs Mais que li raut? ('hose est aperte

Que par li est alés a perte.

..Lase, eaitive! dist la mere,

Con sui ceile en grant misere!

Desconfortee sui por roir,

7wn (pmant ensi ai perdu mon oir."

L'enpereïs est mont dolente.

Bien pöes croire une s'entente

En maint point se diversefie,

Qn'ele set bien qu'ele est traile,

765 Mais ne s'en seit pren escuser

Et al sospir et al plores

s'est prise, car n'en pot el faire

Li traitre, li deputaire

Des lous cris of des hantes vois

77 s'est esbahis. Sus! est sa lois.

Sus est sillilis innelement,

Si a plorei mont faintement.

„Ahy! fait il, quel traïson!

Con par a fait grant mesproison

775 Qui mon neven m'a chi ocis!

Lecerese d'autre paìs,

Por coi lavés rous a mort trait?

Aillors avẻs ros el mesfait, 
('ar rhi nel eommencastes pas.

7xn Vos estes prisp el lin .Julas.

s'en deris estre a mort traitie.

Aillor: fustes ros ja jugie,

() ue chi por rostre felonie

Forfait aris menlnes et rie.

ins ('ar estes ros, bians sire freme!

firn sui oncles et rous li pere.

Arse doit rstre del forfuit."

l'ar jugement l'a bien mestinit

Li traître, li souluians:

79n A soll conspl a ne sai quans

Qui volentiers arse l'eüsent;

Ja pitié mule n'eu eüsent.

Mes li sire par sa noblere, (yui si le voit eu grant destrece

795 lit amener l'i atroit,

A dit que ja ne sonferoit

K̈en son païs fust essillie.

La dane et tote sa maisnie

lin merciierent lor singor:

(F,135 roa) sin ,Nire, mout faites grant honor,

('ar chi aroit powe vengance,

s"en seroit vius la ramenbrance."

li sires li pardone mort,

Hener l'en fait i m sien port.

sils the nes i ert arivee,

As marouiers l'a enmande

Quil le mainent en sus de lui

Ft sans moleste et sans anni.

hat dame pleure temement,

s.0 Entre en la mer, si ont boin rent, (F, $135 \mathrm{r}^{\circ} \mathrm{b}$ ) 850

sacent le roile, en hant l'ont mis:

La nes estonge del païs.

La dame prie et pleure ef brait.

„Lase! fait elle, sans fortait

815 sui a grant tort escaitives

Et mise fors de ma contree."

Adont deront sa bele crine,

Et eil qui l'ont en lor saisine
Pensent tot el quiele ne cuit,

x20 ('ar il covoitent le deduit

De la dame por sal bianté

Et con cuu'ele a adés ploré

L'aroit amynes descolorie.

Dolante et mal aparellie

exs siet en la mef. Li maronier.

li vont hardiëment projer

("or se conlont, si ne plort mais:

Il l'ameront bien et en pais,

Por qu'ele face lor tatent.

*31) Et, quant la dame ot et entent

Qne elle ciet de mal on pis,

Dont li retorble, ce m'est ris,

Et ses pensers et ses corages.

"Che seroit ore grans outrages,

8.5. I ist la dame, yue vos feriés,

Se rous ensi me honissies;

Che n'ert ja, tant con soie vire.

Se jou ichi sni or caitive,

Ne me devés querre ma honte,

nin N'a moi n'afiert, n'a ros n'amonte."

„Feme, amesure ta penspe!

$\mathrm{U}$ tu seras en mer getee,

U tu feras nos volentés.

- Encore soit parfons li gués,

845 si aine jou mios que jon i nie

K'o vons remainge par folie.

En mer sui, et amerement

Qnerés ma mort et mon torment,

Et, se m'anor querés en mer,

En tel maniere a mont d'amer.

En mer n'ai soing de tel misere, S'aine miols en mer la mort amere

Q'en mer me taciès tant amer;

K'amerement vinc en la mer.

s.5 Faites ent tot rostre plaisir,

Car jou ne voel pas consentir

A vos dont jon tel cose face

Dont jou vers Dieu trop me mesface.“

787. Ms, des forfait. -807 . Ms maigent. - 814. Ms. Ladase. - 837. Ms. en vie. - 857. Ms. del cose. 
Cil voient bien que c'est por nient.

860 Pres d'une roce la nes rient;

Li maronier l'ont iluec mise,

Nule pités ne lor est prise

De la dame, qui la remaint.

Mout se dolouse et mont se plaint,

s6.5 ('ar n'i a mule garison.

Qui lor's veïst de sa façon

Com'or est pale deremue!

Sole est et solement se tue,

Car tot adés sospire et pleure.

s7w „Dens! dist elle, trop me demeure

Vostre secors, que j'atent whi.

Doce dame, vostre merchi!

Proiies por moi a vostre til

Que il me get de cest peril!

875 La roce est mout estroite et haute,

Et de tous biens i voi la fante.

Se je par vous n'i ai secors,

Il ne me puet renic d'aillors.

lceste lase, escaitivee,

s8o Se elle fist an la contrer

U il eüst ancume gent,

Dont n'alast pas si malement!

Mais or sui sole, sans confort.

Cist maronier m'ont mise al port

x*5 $\mathrm{U}$ jou ne roi se peril non.

$\mathrm{N}$ 'i esgarderent pas raison

De la penture de ma vie;

Et si l'aine miols que lor folie.

Quant jou regart environ moi,

890 Nul boin consel n'i truis ne voi.

A tel port sui, lase, eaitive!

$\mathrm{U}$ jon ne truis ne fons ne rive.

Dame, por Dieu, c'or m'entendés,

Qui tos peceors secourés!

895 Je proi vo dous fil qu'il me gart.

Dame, tant ai de vostre part

Que jou le soefse volentiers.

Ne mesaise ne enconbriers
Ne me poroit de rous partir.

(F, $185 \mathrm{r}^{0}$ ) soo Tant vos aine et tant ros desir

Que messaise que j'onques aie

De ro service ne m'esmaie."

(Dames, chi devés vos entendre,

Car boin exenple i pöés prendre!)

90.5 Li gentis fame est en grant paine.

Trois jors i fu de la semaine

K'ains n'i manga n'onques n'i but

Ne nul confort boin n'i reçut

Inse'al tierç jor que s'endormi.

910 Taut ot a Dien crïé merchi

Et a sa Mere donce et piue

(r)ele li fist mout boine aine:

(ni Diens aime, s'a boin confort.

La Vírgene, entruẻs qu'ele se dort,

915 S'en vient a li, si le conforte,

Car tel novelle li aporte

Dont elle avra joie et pardon:

Deservi a boin gueredon,

Et la paine (qu'cle a trovee

92n Li ert mont bien geredonee.

La roce tote resclarei,

Quant tes biantès i desiendi

De la Roine Giloriëuse

Por conforter la dolerense.

\$25 „Feme, dist elle, entent a moi!

Por ta deserte et por ta foi

l'en mariage as bien gardee

Ne seras mais nul jor temtee,

Lt tot li mal que on t'a fait,

930 Tot li peciet, tot li tort fait

Ert a poi d'ore descovert

Voiant la gent tot en apert.

Desos ton cief gist tes grans preus:

Une herbe $\mathbf{i}$ a boine as lepreus,

93.5 (que tot cil qui en beveront

Certainnement gari seront.

l'ense del dinguement reçoivie,

El non mon fil le done a boirre!" 
A tant s'en part, cele s'esirlle, 941 Lisbahie est de la mervelle;

A orisons s'est jluec mise.

lierbe enclli et si l'a prise.

L'us gans aroit, eels en empli;

Mais onglnes mais parler n'ui

945 D'erbe yni si treshele fust

Ne tant bele color eiist.

L'erbe a cuellie liëment.

Es ros a tant isnelement

Maronniers qui par mer guioient (F 135 $r^{\circ} d$, 9:" lice nof et boin mast avoient.

role les voit, ses apela

Et en plorant si lor proia

(une il por Dien a li venissent

Et entre gent le remeìsent.

455 A ancm port cil aresterent,

Ens le misent, si l'en menerent.

Bele persone ert et honeste,

lit por pitie li font grant feste.

A port lout mise; elle s'en ist.

(46) A tere vint ef congiet prist.

Un mesel troeve en mi la roie,

La wentis dame en a grant joie;

le s'arbe a pris, si le triulla

Et de eler vin lo destenpra.

9is (bil ell a but, tous fil garis,

lirases l'en rent et grans mercis.

P'ar tot s'en vait la renomee.

Si s'esmuet tote la contree:

Tot li mesel vienent a li,

y7o T'os lis sane, tot sont gari.

li fel yui ot son neven mort

lit (fui jugie l'ot a tort

Estoit meseans, si ot ret scut

$\mathrm{K}^{*}$ en celle terre une dame cut

975 (2ui les malades garisoit;

Et se's freres. (fui mont l'amoit.

(zui sires ert de la cité,

(Quant il en sot la reriti

K'me tes feme est arivee,

gxu si lai plus tost quiil pot mandee:
Ofert li a de sou avoir,

Bel le reçit a soll manoir.

(quant elle $\mathrm{i}$ rint, a sa venue

List la eités tote esmeile.

axs Nins vel connt, et si fu ja

En tel point que mont li greva

lij jngemens e'on sor li fist

A tort, car alle nel fortist.

$\mathrm{Li}$ sire docement li prie

9:m (que por avoir ne le laist min

()n'ele son frere ne garise.

„Ja certes, sire, n'j reuise,

Fait la dame, por vostre avoir;

Mais nequedent, s'il violt aroil.

995 Thel mal qu'il a sa garison,

Si face sa confesion

Vraiement de tos ses preiés

Dont li siens cors est enteciès.

De devant moi roel qu'il le lace,

(F. $135 \mathrm{v}^{\mathrm{a} a}$ ) $1000 \mathrm{Si}$ qu'il me roie face a fice,

Et set persones $\mathrm{i}$ seront

U moi qui le confesseront.

Vos et ma dame, che sont deus,

si resoient aroegues teus

1005 (2ni sou peciet sacent cele]:"

('il se prist mont a vergonder,

Grant il oil que entresait

seroit confés de son meslait,

s'il entresait riolt garis estre.

1m10 $\mathrm{Li}$ sires amaime le prestre;

Envijon lui sont tot asis.

De ses peciés, çou m'est avis.

En partie se coufesa.

Le murdre de l'enfant cela 1w25 ("u'il ot mordri. La dime l'ot,

(Qui l'aventure mont biens sot.

„Amis, fait elle, trestot di.

('ir autrement, jou le t'afi.

Ne te poroie joll garir, 1020 se nulle riens me vios corrir.

- Frere, por Dien, che dist li sire.

Puis yue trestot te eovient dire, 
Ne fai dont nule couverture.

Se mulle riens as en ta eure 1025 Que tu lioses dire por moi.

Jel repardoins en boine foi."

Li meseans l'ot, s'a sospiré

Et plaint ançois et s'a plore

Et en plorant a rejehi

1030 Confaitement I'enfant mordri:

La traison tote lor conte;

Et li frere[s], a cui fou monte,

si en pleme mont tenrement

Et si regrete mont sorent

1035 La boine dame qu'il perti:

..Mar l'acointai et mar le vi,

Car forjugie fu a tort!

Plus miest de li qute de la mort

Mon fil, qut mes freres ocist

10£0 En traïson, si comil dist."

Che dist li dame: "Tes me chi!

Or arés bien le tort oï,

si m'est mont bel que le sarés,

Et neqnedent cis ert sanés

10.5 Qui porcaça le trä̌son."

Elle li dona garison

Par l'erbe boire, et cil respase;

Et li sires, qui arant pase,

Li prie mont qu'ele remange

(F, 135 $\nabla^{\circ}$ b) 1050 Et que son frere a singor prende.

('elle ne rolt, ains s'en parti,

Mais li sires et si ami

L'ont mout conduite liëment.

Elle s'en parti esranment;

to5s Les malates qu'tele voit sane.

Tant a ale k’a le cuinsaine

En est droit dedens Rome entree,

Que ele avoit mout desirer.

En la rité se herbrega,

1060 Plusors malades i sana,

Qui mont sont liẻ de sa venue.

La dame s'est desconneĭe,
Si que nus hom nel conisoit.

Par la cité sovent aloit, 1065 Les enfers metoit eu sante.

li citeain de la rité

Le ront noneier l'eupereor.

Qni a grant ire et graut dolor

De son frere, qui meseans est.

s070 II li'a exure de nul aqnest.

f'ar l'empire, qu'jl of ronguis,

A il perdu, cou li est vis,

quant cesui pert por cui amor

Il amoit plus tote s'onor;

1075 Mais la dame ot a tort traï".

Mais li grans maus yui le desfie

En a cruël vengance prise:

Si le destraint, si le justice

Qu'il porist tos dedens le cors

10so Et art derlens et art defors.

Li ardors et li grans rancure

lue fait peuser a porreture.

Lienpereres of la novelle

De la dame; mont li fu bele.

108. Mandee I'a, celle est renue,

Eit l'enperere Ii desmue

L'ocoison por coi l'a mandee.

"Dame, dist il, buer fustes nee,

Qui tel grase en eest siecle avés

Inow the ces lepreus que ros sanes!

l)ame, un frere mout est atains:

Por un petit (qu'il n'est estains

lel mal qui le destraint si fort:

Ades ell est dusk'a la mort.

1095 En vous est, lame, del garir.

Pros ert apres a vo plaisir

lel geredon et del loier:

Se vostra herbe li a mestier,

Ja n'en sarés tant demandè

$\left(F, 135 \mathrm{v}^{\circ} \mathrm{C}\right)$ s100 Ne vos en voelle plus doner."

"Sire, con pöés bien saroir

Que jou n'ai soing de rostre avoir. 
Je l'ai de Deu, et de Dieu l'ait!

Faites li jehir son mesfait

${ }_{105}$ Et son pecié sans nul delai,

Et, s'il i faut, jou li faurai

De santé et de garison,

Car saus rraie confesion

Ne le poroie pas garir.

1110 - Dame, trestot a ro plaisir

Sera fait, dist li jovenencians,

„Li garirs mest et boins et biaus.

Riens en cest mont plus ne coroite

Na ear's, qui tant par est destroite."

1115 ,Anlis, por vostre bien le falç.

Oiant l'apostole diras

Et chi deraut l'enpereor,

Qui dolans est de vo dolor

Qne rous en vostre cors sofrés

${ }_{1120}$ Tos ros peciés a moi rendés!

- Dame, fait il, mont volentiers!"

L'apostole manda premiers,

Puis les senaus, o els son frere.

Devant aus tons dist la misere,

1125 La traïson et la boisdie

Conment sa dame avoit traie.

$\mathrm{Ni}$ laisa onques riens a dire

Por la santé que il desire.

Quant l'enperere ot et entent

1130 Qu'il of ovré si malement,

L'enperreïs regrete et pleure.

„E! las, fait il, a con male eure

Mes cors del sien se departi,

Car mellor dame onques ne vi!

1135 Mout ai mesfait, ne sai uque dire.

Ceste arenture est asés pire

Que ne cuidoie endroit de moi.

Mesarenu m'est mout par toi,

Frere, quant l'ai ensi perdue.

1140 La mellor dame m'as tolue

Que gentis hom puist mais aroir

Par hautece ne por aroir."
T'ot eil qui la sont environ

Pleurent son cors et sa façou, 1145 Son seus regretent li plussor,

S'oneste rie et sa valor,

Et dient tot: ..Bele mar fu!

Por coi li est mesavenu,

Quant aiue nnl jor ne forfist rien,

(F. 135 $\nabla^{\prime}$ d) 1150 Ains se maintint et bel et bien

la corone de cest enpere?

. Ta mais n'ara li enperere

Si boine dame ne si bele.

Trais Dens! qui en saroit norelle, $1155 \mathrm{Si}$ le mandast qu'ele venist

Et tote l'onor represist,

Car nos ell somes mout dolant?"

Quant elle voit le doel si grant,

S'a dit: „Sengor, car vos taisiés!

1160 Je sui cele que ros plangiẻs,

Qui mainte paine a puis eüe.

La Lien merehi! ehi sui renue

A] point que jou valoie plus.

Deus, li vrais Pere de la sus, 1165 A bieu mostré l'enpereor

Que a grant tort perdi m'onor."

Quant l'emperere ot et entent

Que c'est sa feme voirement,

Grant joie en a, graut joie en maine.

${ }_{1170}$ De grant joie est la sale plaine

Des gens qqui la roïne oirent;

Mais por niënt s'en esjörent:

Ja mais lor dame ne sera

Ne le roiaume ne tenra.

1175 L'enperere le violt reprendre

Et tot l'enpire li violt rendre;

L'enperreis l'a refusé,

N'a soing de unle dingnité.

Celni gari et fist tot sain,

1180 Puis si a dit: „Sengor, en rain

V'aparleriés de mariage,

Car jou ai or un autre usage

1110. Ms. iotenencias. 
Entrepris et me antre afaire.

Drois est, si le roel or mais faire:

${ }_{1185}$ Penitance de mes peciès.“

L'apostole ceï as piés

Et mont li prie et le conjure

Sor Dien et sor tote Eseripture

Qu'il le face nonain relee,

1190 Car aillors n'a nulle pensee.

Lienpereres le violt desfendre

Et, quant il voit k'a şou violt tendre

Et c'aillors nel puet amener,
No le vot mie destorber.

1195 L'apostoles le saintefie,

Puis est entree en l'abeïe.

Tote sa rie $\mathrm{i}$ demora,

Iluec fu tant que defina.

El service la Gloriëuse

(F. $\left.136 \mathrm{r}^{\circ} \mathrm{a}\right) 1200$ Fina sa vif presiëuse.

Sa casteé a bien gardee,

N'est mal mise ne violee,

Ançois a si son cors finé

Qne Dameldieus l'a prise en gré.

Explicit de l'empereris.

\section{Alphabetum narrationum d'Étienne de Besançon}

Ns. Paris, Bibl. nat, f. lat. 15913, fol. it $\mathrm{r}^{0} \mathrm{a}-74 \mathrm{v}^{0} \mathrm{a}$.

(Fol. $74 \mathrm{r}^{0}$ a) Regina quanto dignior, tanto magis castitatem suam seruare debet. Ex miraculis Beate Uirginis.

Imperator quidam Romauns uxorem habens pulcherrimam et castam ad remotas regiones pergens, imperium et fratrem summ inuenem regine commendanit. In cuius amore iunenis exarsit et ei cor sum apernit. Sed illa totaliter reunente et illo frequentius instante innenem in turri inclusit et regnum pacifice rexit. Quingme annis elapsis imperatori redeunti frater suns, de turri de licentia imperatricis emissus, occurrit et imperatricem apul eum pessime accusanit dicens eam puplice et cum omnibus fornicatam fuisse. Et quia ei consentire nohnerat, ob hec eum in quadam turri (Fol. if $\mathrm{l}^{\circ} \mathrm{b}$ ) inclusit et ob hee ${ }^{1}$ se jpsum tenmem et pallidum esse dixit. Imperator nimis cito credens nerhis fratris dolore cordis fere exanimis in terram corruit. Resumptisque uiribus reginam ei ocemrentem et em amplexari uolentem graniter perenssit et ad terram prostranit. Precepitque dnobus famulis, ut eam ad nemns ducentes decollarent. (ui ad locmm decollationis pernenientes eam cognoscere nolnermnt. (lamante illa nobilis quidam cum multis superueniens illis interfectis reginam liberanit. (Quam nxori sne commendauit et fili sui unici paruuli nntricem constituit. Frater antem domini, miles, inflammatns illins amore et ab ea pluries repulsns nocte illa dormiente parmuli inxta eam dormientis gntmr entello secanit et in mam illims dormientis cutellum relinqnens abiit. Sanguine igitur pueri calislo per lectum decurrente excitatur illa, et ad eins clamorem excitantur dominus et domina et tota familia. Qui illam interficere nolentes prohibiti sunt a domino et domina precipientibns, quod nantis traderetur in longimpuam regionem ducenda. Cuins amore capti nante, cum eis consentire nollet, eam submergere nolnerunt, sed Domino consilium eorum mntante in alta rupe posuerunt. Vbi Beata Uirgo ei apparens cou-

\footnotetext{
1 hec manque dans le ms.
} 
fortanit. (am mounitque, ut herbam. que erat sub eapite suo, reciperet et custodiret et inde omnes leprosos mudaret. Thunseutibus antem iloi quibusdam natis clamanit. (pui ej condolentes ad portum adduxermut. Que statim ibi leprosum simanit. Impins antem ille, qui fratris filimu ocriderat, lisprosus fuerat linetus. Ad quem crmi ipsa introducta esset nee ab aliquo de familia eognita rogataqur a domino, ut fratrem ums euraret, respondit se non posse facere, nisi prius in presentia sui et . Yll. personarum omnia poreata sua confiteretur. Ille rero ommia alia pereata confitens illud. quod de ea fecerat, tarebat. Tamdem tremens a fratre asserulatus totnm rerognonit. bicente antem illa: "Ego sum mulier"* instabant, nt rnm in uirum

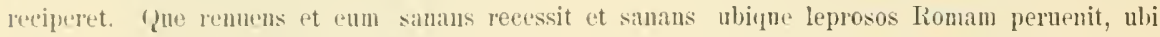
fratrem uiri sui imperatoris Jeprosum prins pecuitum sum, ¿noul in eam commiserat, confitentem ("unetis (Fol. $74 \mathrm{r}^{\circ} \mathrm{a}$ ) Hentibus de tante lomine amissione se ipsam eis manifestans sanauit et at papa, quia notum in tribulationibus leo feeerat, habitum religionis accepit et sancte uitam fininit. (Hoc est videliect ad infumiam et Mariam).

\section{$\mathrm{K}$}

\section{Johannes de Garlandia}

Stella maris de Miramelis Beate Marie Tirginis

(Mis. Londres, Brit. Mus., Roy. 8. C. IV, fol. $18 \mathrm{r}^{\circ} \mathrm{b}-15 \mathrm{v}^{\circ} \mathrm{b}$ )

$-\cdots-\cdots-\cdots$

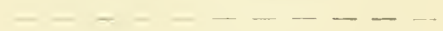

I

(F. I8 $\mathrm{r}^{\circ} \mathrm{b}$ ) Imperatrix acusatil

Rome fuit et dampuata

Livoris aenleo.

Lepras curuns pauper vixit,

Virgo elemens ut predixit,

liffectu gramineo.

\section{Il}

(F. $\left.18 \nabla^{\circ} a\right)$ Lepral lesos delatores

Expurgarit et honores

Jumli, celi meruit.

Dolus tandem apparebat,

Et ad trmpus yne latebat

Veritas emicuit.

IV, 3: Ms. Celeris
III

Frater hanc imperatoris

Esse Joco corruptoris

Optans acusaverat.

Hane in silram spiculator

Duxit, illam sed venator

Liberans salvarerat.

\section{IV}

Nutrix fit hee, sed anatur

A quodam, quo perpetratur

Sceleris andacia:

Puerile resecabat

fiuttu et hanc acusalat

Invilia resania.

\section{V}

Rursus casta condenpuatur, sed a nautis conservatur 
In navis regimine.

Nantis illa stans rebellis

Est relicta in rupellis

Dei sub tutamine.

\section{YI}

Mater, Christmm que portavit, Herbam illi demonstrarit. (Qua lepras mundaverat.

Vixit arte medicine, Hostes suos post in fine A lepra sanaverat.

\section{VII}

Lepra cedit leonina

(F. $18 \mathrm{v}^{\circ}$ b) Sua ricta medicina,

Cedit elephancia,

Phthiriaque condeletur;

Condeleri perhibetui

Putris alopicia.

\section{VIII}

Sicca prior exardescit, Sertens friget et areseit, Friget, hnmet tercia, Quarta calet et humescit; Tamen eger convalescit Maternali gracia.
IX

Sanitas non potest geri, Nisi prius confiteri Velit hic, uni patitur. sic infantis iugulator Est peccati demonstrator Et simus efficitur.

\section{X}

Fratris hec inperatoris

Lepram sanat, delatoris Confitentis scelera.

Imperator ius nxoris Papam petit, sed pudoris Illa vovit munera.

\section{XI}

Vitam sanctam terminavit, sed ad celum transmigravit Papali consilio.

Hec est mptis in exemplum, Ut sint sanctum Dei templum Casto sub coniugio.

\section{XII}

Castitatem hec dilexit, ('asta mater' quam protexit Nec castam deseruit.

\section{L}

Version de la Vie des Pires

(Ms. Paris, Bibl. nat., f. fr. 1546, fol. $29 \mathrm{r}^{0}$ a $-33 \mathrm{r}^{0}$ a)

Du roy qui ala outremer

(F. $29 \mathrm{r}^{0} \mathrm{a}$ ) Follx est rui acroit seur ses pianx. T'andiz comme li gens est bians, Doit l'en son cuer batre et plessier
T'our le jeu foür et lessier. 5 Ne me merreil, se l'en mesprent; Nes qui par coustume s'i prant 
Si se doit pour musart tenir,

Que l'en sce doit bion maintenir.

(Horer et bien fere derons

10 selone ce que les jours avons.

saint lol, qui fu de bone escole,

Nous dist une hone parole.

(quant il fu enfes, si savoit

('e yu'a enfance comvenoit

15 lit si parloit eomme petis,

(Qne pas n'estoit de sages dis;

Et, quant d'ome ot et torme et cors,

In lui ruida l'entance hors

Et fist ce yula home comrint,

20 Quant d'enfance a home revint.

Ensi se doit rhaseun prover

Et selone son aage ourrer.

A s'enfance rient et retourne

(Qui a bien fere ne s'atourne;

(F. $29 \times$ b) 25 Enfe's de cent anz soit houniz,

(u) taut se tient en ses deliz

Que mort de son bien le demet

Et a dampulteion le met.

Haï! con se repentiront,

30 Quant al danpnacion iront!

Et cil qui ont tant espargnié

Que Paradiz ont gaengnié

Seront a repos a touz jors.

('il est maleiirez et glous

35 Qui de son aise tant se poine

(2u'il se met en torment et poine.

Preus est et de sage ahatie

(uni par example se chastie

Et yui chastïment ne crient.

40 La honte et le mal qui l'en vient

Ne porroit pas nus hous descrirre;

Ne langue ue le porroit dire,

Lo mal ostel que l'en li fet

Et apreste pour son meffet.

4s Aprenez rous, gens, aprenez,

Qui ci de leger mesprenez:
Il fet bon savoir et aprendre

('e par yuoi l'en doute a mesprendre

Et par quoi l'en eschire honte.

50 ('i enpress vons devis un conte,

Estret d'estoire et de miracle,

('ertes yne des le tans Earacle

Plus biaus ne fu mes racontès.

()r l'orrez, se vous l'escoute\%

55 Jadiz en la terre de Rome

Ot un enpereour preudome.

Bien tiut terle et bien justissa

(Yuant que a li se herissa,

(i yne maintes gens le cremoient

go Assez plus que il ne l'amoient.

Une fame ot de bon parage,

Bone, cortoise et preus et sage;

Mes li biens qui en lui estoit

Toute richesse semmontoit:

65 Dien et sa Mere et Sainte Eglyse

Ama la dame sauz faintise.

Ses sires l'ama mout et crut,

Et elle lui tant com'el dut.

De rraie amor et par cremor

70 Ama et servi son seignour

(Car nus n'aime bien, e'il ne crient;

Bone amour par cremeur se tient),

(F. $29 \mathrm{v}^{\circ} \mathrm{a}$ 'T'ant que li enperieres ot

Maladie, si qu'il ne pot

75 Lever, aiuz jut mout longuement

Et malades fu durement,

Tant qu'au Sepucre se voa,

Si come ses cuers li loa;

Et promist a Lien qu'il monvroit,

ro si tost con respasse\% seroit.

Nostre Sires, qui ses bras tant

Au pecheenr qui se repant,

De l'enfermeté le geta

Et de la mort le respita

s5 Pour sa bone contriction

Et qu'il i ot s'entencion. 
A son vel briement aquiter

Fist tantost son oire aprester, Come il en sa senté revint.

$90 \mathrm{Si}$ comme a tel home couvint,

Pour soustenir lui et sa gent

S'esmut garnis d'or et d'argent

Et de chevans et de harnois;

Nout s'en parti a grant noblois.

95 Un cheralier a frere avoit

Qui sa terre et sou droit tenoit;

A lui essancier mout penssa,

En son bail sa terre lessa,

Par tel maniere que sa fame

100 Fust deseur tous mestresse et dame.

Et elle si fu tout en pais,

Bone ful avent et aprés;

Contre les felons s'engressa

Et vers les humbles se plessa;

105 Tous jours se tint en verite

Et ot avec lui charitè.

Ses serourges mont la servoit

Et comme sa dame l'amoit,

Tant qu'a l'Asencion avint

110 Que la dame a Rome court tint.

Pour l'amour du jour, sui fu haus,

Li tans fu biaus et clers et chans,

Et la dame fu gente et bele

De cors et de conleur novele,

${ }_{115}$ Qui lui ot entlambé le vis,

Si wue bete fu a devis

Que pour le chant que pour natmre,

Qui en lui fere ot mis sa cure.

Tant que les napes mises furent,

${ }_{120}$ Si s'asistrent si comme il durent.

(F. $29 \mathrm{v}^{\circ} \mathrm{b}$ ) L'enpereris s'asist premiere,

Son serourge devant sa thirre

S'asist, et mengerent ensemble.

Amours, qui as foux le cuer amble

125 Et justisse par fol roloir,
Le fol serourge fist doloir,

Qu'en son cuer sa biaute bouta,

'Tant qu'au fol roloir se domna.

II la rit avenant et bele,

130 'Tant qu'a son cuer en rint novele,

Car ensi come foux se print,

lo l'amour sa serourge esprint.

De lui regarder se disna;

Con plus la vit, plus s'enclina

135 Ters lui ses cuers et la vosist.

Riens qu'elle fist ne li messist,

Car de ses eux la regardoit;

Mout li sist quant qu'elle fesoit.

Quant les tables furent levees

1*4 Fit il orent leurs mains lavees,

(il a son ostel s'en tom'na

Et de la court se destourna.

Tont mua son fet et son estre

Pour l'amour de son novel mestre,

${ }_{145}$ Qui en son cuer fu herbergez.

En po de tans fu estrangez

D'uns et d'antres, car compaignie

Ne le solaz ne li plot mie.

Tous jors voluit penser a cele

150 (Qui li ot mise l'estancele

El cuer pour quoi si se doloit

Gue riens fors pensser ne voloit.

Qui aime si se doit pener

I'estre en joie pour obbliër

155 L'amour dont il se plaint et deult,

Car, quant plus la roit, plus la veult.

Ensi li foux queroit sa mort

En ce qu'il tenoit a confort.

Une nuit a cele peussoit,

160 Pour fol se tint et dist: „Or soit:

Je l'aing. Voire, fas je reson?

Nanil, voir, mes grant mesprison,

Qui bee a mon frere homnir.

Me doit donques bien avenir,

89. sa manque dans le ms. - 91-2: Ms.: Sesmut garnis lui tet sa gent Pour soustenir lui et sa gent (Les corrections sont faites d'après $t c$ ms. Paris, Bibl. nat., f. fr. 15it). - 124. Ms. au foux. 
165 (Quant je veil traï et forfere ('elui yni bee a moi bien fere?

Nanil, se ne puet avenir.

Mon fol talent mestuet gerpir;

(F. 30 ra) Mon fol talent, ma resverie.

170 (quai ge dit? .le ne lerai mie.

('oment lerai ge lis meillor.

soit pucele, soit a scignor,

(yni oncues fust de mere nee?

Ju renit que loieus la m’a donee

175 lit verult que la bele soit mole

lit yur je ses bons amis soic.

se li voloirs en est vilains,

Li ruers en est de joie platins.

Lien doit et pene et mal soufrir

1no Pour si bel solas aconpliz.

Birn est enpluiere la poine,

Yui a parfection la moine.

Bien croi yne ma poine arendra

Et que mes voloirs s'i tendra;

axs Tonques doi ge ma poine amer,

s'a moi me rent douz pour amer.

Th: l'anertume ne me chaut,

Amour saus poine ne me faut;

Nliens vaut, quant elle est bien penee

190 (Qu yuant de leger est donee.

Mes, fous, que vas tu devisant?

("est devise et panssel denfant.

Autel faz je. Je cuit tenir

Ce cu'il ne me puet avenir.

195 Bien sai qu'ol ne mameroit mie;

Amer' ue courient sanz anie.

Ele se leroit ainz deffere

Quialle rosist tel chose fere;

si mo convient mon ener desdire,

200 Et morir m'estnet a matire."

Tant se plaint, tant se dolousa

Pour rele whe tant goulousit

Que du tont au lit acoucha.

Mont fu pales, mont desfroucha.

203 De toutes aises s: mist hors;

Pour ce vint an neant du cors. linpereris, qui mout l'ama, Qui tous jours frere le clama, corrouciee en fu durement.

210 Hout le risita bonnement,

T'ant c'un jour deraut li se sist

Sanz comprignie, si li dist:

"Biau frere, se vous le savez,

bites moi quel mal vous arez.

215 . to vous di bien que pour avoir

Ne vous leroie mal avoir

(F. $30 \mathrm{r}$ 'b) Ne pour rien que fore prisse

On je m'onour saluver seïsse.

I)ites le moi seürement,

220 Et vous arrez conseil briement.

Pour 'pue l'en le puisse trourer,

Ja denier n $\mathrm{i}$ seroit arer.

- Dame, je ne vous sai the dire,

For's tant gque vous arez le mire

22. Ponr qui sainz et haitiez seré,

On je ja u’en respasseré.“

En sospirant laz se clama,

Plus ne pot dire, ainz se pasma,

Et cele le chief li soutint,

230 Tant qua son memoire rerint,

Si li dist: .' 'ar me fetes sage,

Sil vous plest, de vostre malage,

('ar nule e elee n"i roi.

— Te vous aing, dame, plus yne moi.

23.5 - Vous miamez? - Voire. - En ¿quel maniere?

- Pour I)iet, dist il, ma dame chiere,

Quant vous uel savez, si sachiez

l'our vostre amour sui acouchiez.

T'ant rous covoite et tant vous reil

2*0 Q Que je di que morir me rejl."

('ele, qui ama lrïuté,

Tit le musart desconforté,

si ne le rolt pas desconfire;

Eu sousriant li prist a dire:

245 ,Lerez sus, si vous confortez

Et de cest mal rous deportez,

Qu'en petit d'eure vient grant biens: 
Je suiz assez rostre et rous miens;

Par droit nons devons entramer

250 Et li uns l'antre amis clamer."

Qui sanz doner a fol promer

De neant en joie le met;

Ensi cil de noiant fet joie:

Plus fu sires que raz en moie.

255 La dame d'illec se parti,

Et cil maintellant se resti.

Ainz que li quins jours fu passez,

Fu il gueris et respassez;

Par le bian respons cuida bien 260 Aroir tout fet, n'il n'ot fet rien.

Quint cele rit, si la requist,

Et cele maintenant li dist:

"Qu'est ce? Que penssez vous a fere?

Me ruidiez rous a honte trere

(F. 30 v$^{0}$ a) 26.5 Pour rostre lechoiz aconplir?

T'aisiez! Ce ne puet arenir.

Mieus vondroie estre arse en un feu

Que je forfeïsse a mon vell

N'a rostre frere, mon seignemr,

270 A qui rous gardez mal s'oneur.

Quant de tel chose m'apelez.

C'est oisense dont rous parlez.

Sachiez, se plus m'en parliezz.

Que mes anemis seriëz.

27.5 Cil. qui el cuer ot la folie,

Sil requeste nonblia mie

Pour rjens que la dame deäst

Ne pour semblant qu'elle feïst,

Ainz la requist hui et demain;

$2 * 0$ Mes sa priëre fist eu vain.

Taut li dist et tant l'empressa

Que cele sur lui s'aon'si.

Bien vit qu'a lui ne durroit pas:

Prendre le tist isuel le pisc

2s. Par force et metre en une tom

(tui fin fermee en un destour.

Pour lui garder grant garde mist
Et richement servir te fist

De. ce cqu'al home couvenoit

zми (qui malgré sien prison tenoit.

Longuement i fist son sejour,

Tint que l'eupereris un jour

Sot que reuperiere renoit

Et que de Rome pres stoit.

295 Mont en fu liep durement,

son serourge fist erroment

Delisrer, qui tantost monta

Cone cil qui mont se donta

Pour son meffet, dont encourper

: wo Toloit cele et lui deliverer.

Ensi le deable le fist.

A son frere rint, si li dist

De sa fame que tele estuit

Que parler a hil ne devoit

305 Et que sa terre avoit honie

Pon' despendre en sil lecherie,

Et li dist qu'('n prison l'ayoit

Tenu. pour ce qu'il ne roloit

A sa lecherie partir

31 Ne sa maurestiẻ consentir.

Cil, qui sa fane ot mont amee,

Quant il oi la renonmee

F. $30 \nabla^{\circ} \mathrm{b}$ (Qn elle avoit tenu en prison

son frere pour sa mesprison,

315 Si crut le serf plus de leger.

En lui n'ot riens que couroncier,

Tant que de loing la vit venant,

Grant joie pour lui demenant.

La chiere mie renbruncha,

320 Grant erce vint, si lapela

Et dist: ..Bien soiez rous venuz,

Ii miens sires et li mirus druz!

- Et rous soiez la mal trovee

c'ome ma desloiaus provee!"*

.12.; Lor's commenda quelle fust prise

Et a mort pour son forlet mise.

("onques n'ot lesir de plus dire 
Yespare de lui cseondire.

Mitutruant lin prise et minese

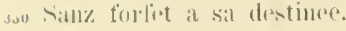

'Trois dhealier's senr lat marinte

loing de gent en mere grastime

La menerent pun metris a mort.

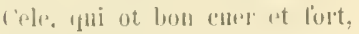

3: Diens reclama mout domerment

Quil la gardant de dampnement.

"Ever, tist li mus, je ne puis. voir?

- Ve je rertes, pour nul avoir.

Qui me tlevoit coper le rhiel'.

stu Trop me seroit a liege grief.

kt, sel n"i muert, pour li morron.

Or vons dire que nos lexom.

Fist li mes, yui la volt samver.

('as la menon en rele mer.

345 A la roche ou miss me reperes!

sie vous elsi le vole\% lere.

Ja mes jour parler n"en ormons.

Ensi nous en aguiterons.

Car trois jours nï prora duret

350 le les grams omles endurer,

se lamediens ne la sesustirnt,

Qui tous les sirns ganle et maintient.

Bien la puet samver, s"il li plest."

A itant lessierent le plet

3 is lit a ce fere s'acorlerent.

A cele roche la menerent;

sanz robe fors de sa chemise

Fu la dame en lat roche mise.

Et "il a liome retornerent,

s60 La mort de la dame ronterent

(F. $\left.31 r^{0} a\right)$

lit distrent qu'il l'orent ocise.

Et cele, qui fin a la bise

Et as ondes qui l'asailloient,

Qui desenr le cors li sailoment,

36: Damediens de ener reclama

fome cele qui mont lama.
Wh "roiz senr la roche se mist.

In bon du cuer plora et dist:

..Jhesu lrist, Pere, pui sanvas

370 Silnt lanièl et qui gardas

Sainte Suzane del tesmoing;

bian sire, qui tiens en ton poing

'Toute poissance a ton taleut:

Garke m'am de denpnement!

375 Gloriënse Pucele at sainte,

Par yui nostre mort est estainte;

1)ame, gue tous tans ai amee

lit comme dame reclamee:

so je vous ai de riens servie.

sжo Garentissioz issi ma vie

Lt le rest blasme me getez!

Dame. si con rous bien savez

Que je n’i ai compes ne tort

Fin re dont mise sui a mort;

dos Dime, sainte Roüne fine.

La uni bonté point ne define;

Roïne, qui enluminez

Et en droit senticr ramenez

Les orlelins desconseilliez

$39 m$ Lit les desvoiez aroiez

Lit fuant pue de bon cher vons prie:

si vous pri ge, dame et amie,

()u rous aiez de moi merci,

si que je ne perisse ci.

39: l'oul' l'e, dame, a rous me demant

Qu a la rostre aïle m'atant."

T'ant fin esploree et batue

De la paom qu'elle ot eile

Qu'on sa priëre s'endormi.

too Amie ot bone et bon ami,

Que la Dame de Paradiz

Li vint devant ell son ariz,

()ui li dist: „Ne t'esmaier mie!

Ie sui la Vere Lien, Marie,

tos (qui par tout en bien te tendré

33:- 3. .Is. La menerent en vne gastine Et loing de gent prour metre a mort. - 336. Ms. Qui.

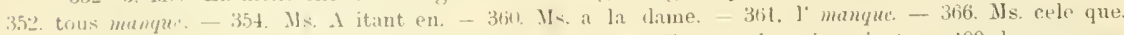
37-. Ms. qui tous tans tai. - 349. Ms. reconseillie\% - 391. Mls. Touz ceul\% qui: prient. - 400. bon manym. 
Et qualeré ta dingneté

Mangé celıi qui t'a ce fet.

(zni elier eomperial son forfet.

(F. $31 \mathrm{r}^{\prime \prime}$ ) Et. quant to te resveilleras,

tio De desons ton chief troveras

Une poingnee d'erbe vert.

si te dire de qu'elle sert:

'Tuit li mesel qui en bevront,

(ài de bon cuer confés seront,

41. Maintenant seront respasse,

Qu'il en arront le col passé,

Et saine char reconverront,

si r'uns et antres le verront.

lles touz jours me tieng en memoire,

t20 si saches me chose voire:

one cil a bone fin rendra

(Yui ma memoire maintendra."

Une nuit et un jour entiel.

lormi cele sanz esreillier,

t25 Et, quant elle fu esveilliee,

Point ne se senti traveillice

De la dolour qu'elle ot rile.

Ainz fn replanie et peiie,

si qu'elle n'ot ne soif ne fain.

t30 L'erbe ru'elle tint en sa main

Mist a sa borrche et a ses enx;

Mont l'ot chiere et si l'ama mient.

Qu’el ne feïst l'aneur de Troie.

Que que de l'erbe fesoit joie,

${ }_{435} \mathrm{Si}$ vit venant me galie

Iterers l'ile de Satlanie.

Qui venoit vers la roche a forme

Ln vent qui la toroit en loche,

si que plaine en estoit la voile.

4tu We hoqueran fete et de toile.

Dames $i$ ot et antre gent.

(uii a un mostier simplement

Aloient en pelerinage.

trant paour orent de l'orage

445 Et du rent qui ai les prenoit,
Qui rers la roche les menoit,

Tant que par force siancrerent

Et la dame sedute troverest.

Mout en liuent esmerveillie;

450 Bien cuiderent estre essillié,

Car de fantosine se louterent.

Tout adés cele part alerent

Et virent rele bele ot simple,

Quj fu ell chemiso et sanz gimple.

455 Hont orent grant pitic de lui,

Que bien commurent som anmi

(F. $\left.31 \mathrm{v}^{\circ} \mathrm{a}\right) \quad$ A la chiere qu'elle of blesmie.

Qui son anni ne celoit mie.

En la galie la mencrent,

460 En merveillant li demenderent

f'omsut elle iert illec venue

Et coment elle estoit si unc.

iele, qui convrir se voloit.

l.ell dist tont el cru'el ne pansisoit.

46: Tout mantenant la revestirent

Et de viande la refirent;

Mout li porterent grant homom.

Et les dames et li seignom:

A son semblant comment lien

470 Quien lui aroit assez de bien

Et que de bon len fu renne;

De plus ne losent comm iïe.

Tantost de lat roche sesmurent.

Bon rent orent ef tant corment

475 Par la droite roie quil timdrent

Qu'a leur pelerinage vimbent

(pinat l'enpereris fin a terre.

Sil chevande ne sot on querre.

te son miens pourchacier ne sot;

tro N"est merreille ulupris ur l'ot.

Quant les dames ot merciëes

Charune plus de reut forees

The lonem que li orent tete

Et du peril dont l'orent trete.

ts5 Dedenz une rité se mist

410. Ms. desus, - 41. Ms. autres. - 483. Ms. qui li. 
Qui desus la marine sist.

A son huis vit une borjoisi

Hinciänue. sage ret rortoise:

Ustel en charite li quist.

490 C'ele pas ue l'en escomdist.

Ainz la herberja rolentiers.

Arec li fu dens jours entiers:

Sa conpaignie hjen li sist,

('ar bien entechier la vist

495 Et hien parlent sanz trop parler

Et rasise sanz trop aler.

Li sires de la terre estoit

Mesians, "fti trop grant plaintu aroit

Ie sa maladie parloient

590 ('il qui couroucir e'll estoirnt.

Tant que cele on oï parler.

lierbe roult a li esprover:

'Font maintenant ele $\mathrm{i}$ alia,

le lerbe a hoive li donna.

IF. 31 v"b) sos dles il fu bien conlés atant.

Quant passee l'ot, maintenant

Fu emrez de senfermetẻ

Et revint dius en sal santí.

si que Dieus et cele loürent

510 'Tuit cil rui lemr seignemr amerent.

Mout fu la dame bien remue

Lt a grant honor receitu;

De tous fin serrie et ameer.

Tel fu de li la renomes

515 (2na lui renoient santé querre

T'uit li malade de la terre.

loaler par tout tant se penal

Que tonte la terre saua.

si que chascun la pousurvoit

52n Et chascun pour sainte l'avoit,

Car le pais enlumina;

lous anz ceste vie meua.

Renomee. qui par tout vole,

Porta de ses oenves parole.

525 'Tant quielle rint a la parsome lusqu'a l'enperiese: de Rome.

(qui aroit un frere mosel:

$x i$ ot leftete char pt pel

Que nus aprouchier ne l'osoit

s30 Pout son cors. qui si fort puoit.

La dame par conseil manda

Ft as mesages conmenda

Que de lamener se penassent

Et que grant honeur li portassent.

${ }_{535}$ Il firent tant qu'il la troverent

Et arec eus l'en amenerent.

A Rome rint mont simplement.

Receite fu bonement.

Mrs onques nus ne la peregut:

5\$0 Ensi ses connoissanz decut.

Qu'il cuidoient qu'el fust timee:

Si se fu bien atapinee

Que pour riens l'en ne labisast,

se l'en senr lui trois jours musast.

5t5 Son seigneur meïmes deçnt,

(qui a grant honour la reçut.

levant le mesel fu menee.

1)e gent $i$ ot grant assamblee:

la pape meïmes $\mathrm{i}$ vint,

s.50 Qui pour sainte fame la tint.

La dame un petit sermon fist,

Jevant tous au malade dist:

(F $32 \mathrm{ra}^{\mathrm{a}}$ ) ,Amis, rous derez bien savoir

Que nus santẻ ne puet aroir.

55.5 se Dieus santé ne li envole;

Et de l'aroir en est la voir

ronfession et repantance

Et en bicn garder penitance.

De par Ilieu tant mentremetré

56. Ih vous quien santé vous metré,

si lo que rous soiez confés

Et bien repantans et en pes.

se vous volez que je rous vaille

Et que mes consens ne rous faille."

565 Il li otroía volentiers, 
Mes ses euers ñi fu pas entiers.

('ar pour un pechié se douta.

Tous ses pechiez dist et conta

Fors celni ynil fist de la tame

57w Ponr perir en cors et en ame:

be son pechié ne gohit mot.

('ele, qui sa faintize sot,

l.i dist: „Amis, ja ñi gnarrez,

Devant que bien confez serez.

57. I)ites tont hors et tout vuidiez

De ce dont estes enterhiez!

-.- J'ai tont dit. - Bien rous i garelez!

Or tenez donques, si bevez!

Par ce boive savrez vous bien,

580 se vons arez oublié rimu."

11 prist le boivre. si le lunt.

Yes onques pour te me s'esmut

De la maladie qu'il ot.

Et la dame li dist tantost:

585 ,Come musart rous decevez!

Dites tont ce que vous savez.

si avrez vostre guerison.

- si diré la grant mesprison

Qui mest dedans le cors reposte,

590 Ce qui de toute joie m'oste?

C'e ne porroit pas avenir;

Ainz veil comme mesel morir.

Ce que je pans je vous diroie?

Desmenbrer arent me leroie.

Et donques ne guarrez vous mie.

- Dé ce ne me chaut, bele amie.

Morir puis, que ja nel diré;

En enfer arent en yré."

De parole sus li corment

600 'Tuit cil runi en la sale furent;

(F. $32 \mathrm{r}^{\circ}$ b) Pape et frere tuit l'apelerent

Et vice de sans le clamerent.

Quant il pour noiant s'amusoit

Et quant sa santé refusoit.
605 Taut th engoissiez qu il leur tist

roment l'eupereris requist

1) the que fire ne devoit.

Et pour re enprisone lavoit.

Et coment a tort la blasma

i21" Et a son fiere lencusil,

Tant que lat tame fist demestre

Et le santé a la mort metre

lour sa desleänté courrir:

..Ensi a tort ai fot monir

61.5 La tine, la bone', l'entiere,

La sage, la bone anmosniere,

(Qui les porres Dien sonstenoit

Lit yni les hons consens dumnoit."

linsi reconnut devant tons

620 Qn il estoit trätres et glous.

Quant il son frere aroit tralhi

Et cele mort et lui homi.

Fusi trois honniz ell aroit;

si dist bien que l'en lo deroit

62.5 Livrer a mort par jugement,

Et il l'otroie bonement.

Quant il oïrent l'errement,

Si se seignerent erroment

he la traisson quil ot fete.

63.3 (Qui ne porroit estre deffete

Pour cele qui estoit finee;

Trestoute kume en fu tronblee.

Quant la novele fin seiie.

Qui guieres n'ot esté teür.

635 Grant en fin la plainte et li denlz,

Et li enperieres tonz ven\%

En son cuer on leva son thel

Et rousist estre mort son reil

Pour sa fame, que tant amoit.

640 Que sanz forfet morte lavoit.

Mes pour ce n'en fist nul semblant

An malade fist maintenant.

Tant que il vint en sa sante.

569. Ms. celni qui. 605. Ns. qui leur. - 627. Mls. (2nt. - 632. Ns. Tonte. - Entre les vers (ift) et

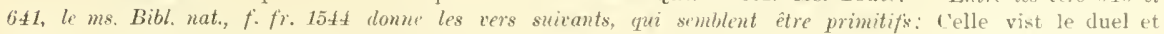
lennuy Que chascun demennoit pour luy. 
Solnone la foi Ji fist bonti.

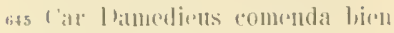

Que l'rul ranclist pour le mal bien

A evelui quni le mal li fet:

lit yui nel fort si se forlet.

F. $32 \mathrm{v}^{\circ}$ a) Mont lis a liome grant li denlz, visu Vinde fu de joir at de jeur

l’ome la dame que mout ambojemt,

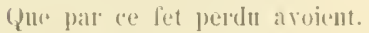

lit rele: qui tont esgarda,

low soi descourviy se garda.

tiss Ansi ronmu pour congié plentre.

Vint a sell mari pour aprembe

Lit volentis de son eomrage.

Belement li dist eomme satge:

"sile (mp)eriser, biem sachiez

we Ite petit est cil tomagez.

puant il pert sa fame on son buef:

f'e comprous ne vaut pas un oet,

f'al re recueve l'en sourent.

Pous une lame en arrez cent.

w65 the vous taut avoir en rolez;

() ne vous ent desesperez.

- .'ai tont perdu quaut que jamoie:

Ma fame, ma terre et ma joie,

Et at esill en seré mis

670 An voloil de mes anemis.

('al pouy sa mort despis et hé

Moi ret ma telue et quant que g'é.

Ensi an neant livrez sui

Pour le colrons yue jai de lui.

675 - Mes sile, l'anizez rous tant

con vous an letes le semblant?

- (Mil. et leje le devoie.

('a j'en lui la meillour avoic

Qui onyurs ful de mere nee."

and Cele se fu destapinet

Fi ot un po leve son ris.

si dist: .. Bian frere, hians anis.

Vor me ri, vestre donce amie,
A qui lliens a salures la vir" 685 Et la Ilame de Mareste.

En qui conduit jai puis sete."

('il l’avisis, si la comut;

1) botrele et de hraz li eorut, si la hesa mont doucement 690 Plus de cent foiz en un tenaut fome ril yui mout l'ot antee, (pui sal perdue of recourree.

sij furent de joie esperduz Qu'i] chatrent tour estenduz 69. Braz a hraz sus le parement. Li apustoiles et la geut (Qui pues furcut la jeleverent Et du miracle lyens loürent Qui mout estoit bians arenus. sm Et par escrit fu retenus.

Et la dame tout maintemant Leur acouta son errement. con la Vierge la secourut, Qui en dorment li aprarut. -15 Et de l'erbe et de la gualie

Toute leur devisa la vie.

'uit cil de Rome se penerent The joie fere. et ell somerent li saint yue il a somer orent

710 Et clere et lai, quant que il porent.

Firent feste trois jours entiers

Fit en rues et en moustiers

kit li emperiere mandia

Les trois a qui il conmenda ins Cele a decoler, et il vindrent,

Gui come mort daupmé se tindrent Pour ce quocise pas n'aroient La dame; poul ce sc doutoient. Mes cil, yui pour anis les ot. :21 I.en' fist (')oistre lemr tie tantost A chascuu do mil mars d'argent, Et les merriat dnrement Ie ce yue il orent forfet; 
Mes el forfet orent bien fet.

725 Et a son lieme fist avoir Grant partie de son avoir.

si l'en envoia soln mients querm.

Et le conjuma de sá ter're,

('ar son estre ne li sist mie;

730 Ensi venja sa felonie.

Et il remest areques cele.

Qui fame fu l)ien et ancele,

Areques sa fame et s'amic,

Qui la bonté n'oblia mie

735 Que la Inume sainte et parfete

Li aroit en lat roche lete.

(Mili l'ot ramenee a s'onor

Et a l'amonr de soll seignor

Et du fans blasme mise hor's.

7+0 Ponr lni nist a escil son cors,

'T'ant l'anor"a, tant la servi

Qne tous ses mentres aservi
Pour gaengnier l'amour de cele

Qui enfenta vinge pucele,

(F. 33 r"a) $7+5$ Si qula la I)eïte devine

Rendi si precrëuse et fine

s"ame qu'es cieus la corona

rele yni grant pooir en a.

(Yni fol envoie lool atant;

75n Qui a traison fere entant

f'ome porre de sims pourchare

('e qui a dampuement le chace.

Nus ne puet ourrer contre loi

Qni ne truisse a pou de delai

7.5 Le gerredon de son ontrige.

Pour ce di an fol et au salge

(que de trair autrui se gart

Et a ce que jai dit regart.

Car traison ef traitour

76. Het I lieus plus qu'autre manlestem:

M

Im vergessen don 17 lieds Frawenlobs Albrecht Paumholezen gedicht Die keiserin von Rom.

Berlin, Kgl. Bibl., Cod Germ. 414, f, $439 \mathrm{r}^{0}-442 \mathrm{v}^{0}$.

I

(F. $439 \mathrm{r}^{6}$ ) ZW Rom do sass ein grosmechtiger keisser,

der hies Octanianus, was ein reisser

des reiches in dy lant so weit;

er dint Got innikleiche.

Der het ein weib, die was so frumm der ere,

las sie daran kunt nymaut nit versere;

ir weiplich er rnd reinikeitt

das kam sie on schwerleiche;

darvmb sie lob vind er pesas.

Vind als vns ('rist geporen wass,

ein herscher aller mechte,

728. Ms. conioia 1?). - 745. Ms, Mi con. - 7t6. Ms, Quen dist.

I, 4: Ms. innn kleiche. - 1, 5: Ms. frumn, 
do nom in vir der keiser lot $z W$ lob vald ar ein walfart $\% w$ volyringe, die was ver mer. zw der er sich gar schnel pereit, auf das er sie verprechte.

II

Fin pruder het der keisser, den ep patte. das er ein neil reigint on seiner statt, pis im Got wider hulff zw lant; er sprach: „Pis sorgen ane!

Ak pal der keisser kam auf meres flutte, sein pruder eilet hin in stiler hutte,

pult rmb die keiserin zw hant; sie sprach: „Dw falscher mane!

(F. $439 v^{0}$ ) Hat das mein her pefolloen dir?

Dein lalscheit hilfti dich nit gen mir. darrmb so las darvone!

Weipliche er die ist ron frot so boch gefreit, das er nit pesers hie den reinen frawen geit; welch den verleust, in hertem stant mis sie frot darvmb thane."

\section{III}

In talscher lieb sein herez so gen ir prane, das er seins pittenss nit mocht abegane, wart ir pedencken lang snd vil, wie sie sich vor im friste.

Zu Rom do stunt cin duren in der statte, darauf pirst mon die purger in dem ratte; auf den gall sie im heimlich zil vind sprach $\mathrm{zw}$ im: "I) iste am pesten rus zw enden das." zom duren er sich fugen was rnd wart sich heimlich lane

dia anf ten imren zicluen, als sie im gepott; zw hant vil sie auf' ire knye vnd tancket (int vor lienden. die in ir aufwil, das sy sein frey mocht stane. 


\section{IV}

Des dages als der keisser kumen solte, lies sie den aus, vor dem sie sich entholte.

Dem keiser er entgegen reit, empfing in schon rod sere.

Der keisser fragt in. wie es da beim stuncte.

Er sprach: "far wol! Dan eins ich dir rerkunde von deines weibes listikeit, die mir pracht schant vnd schwere. Si pat mich in geheim $\mathrm{zw}$ ir; da ich sie nit geweret schir, wart sie mir feint gar dratte.

Zw hant sie mich da auf den duren legen lis, rnd an dem dag, da sie dein zwkunfft west gewis, liess mich ron dem duren an freit. llerck, pruder, die rndatte!“ rud schnf $\mathrm{zW}$ hant der keiserin den dotte: palt must mon sye in fremder wat furen in walt ron dane.

In kaim mon sie im walt vmbpringen solde, vud als mon sie eben enthanpten solde, fil sie anf ire kuye so drat, rnfft Got gar erustlich ane, dass er ir paremberezig wer durch seiner pittren marter er, nud anch Malia reine.

Da sie da kniet rnd Got so hoch rnd diff ermant, ritt vur ein fremmder her, dem do was vnpekant ir cleglich not vad vbel dat.

Der reitt hinzw alleine.

Alss er die keisserin wart onesechen, ruft er sein dinern rnd wart $z w$ in gechen:

"Nempt hin die sehonen mit gewalt, ich mag ir nit lan dotten."

Die diner namens yon der schergen hande; 
der her irsellore anflost ire pande

rnd schwang sie auf sein plert gar palt vnd lint sie hin aus noten, schnit ir gar reiche kleider an, dan ron gepurd rnd ir persan danclst sie in hoch geporen; des hilt er sy in grosser hut rud hohen eren. Des graflen fraw die det ein kindelein geperu; des nom an sich die wol gestalt $\mathrm{zW}$ zichen rnd peworen.

\section{VII}

ler selbig gratti anch einen pruder bette, der legt anch on dey frawen vil der pette. das sic solt da den willen sein, dem sie auch gancz verseitte.

Darvmb er sie so ser hassen pegunde, das er mit nichte ir abseczen kunde;

doch mit we rud mit grosser pein er sie grosslich peleitte.

Einss dages er do leimelich wardt in die kammer fngen sich, da sie lag mit dem kinde.

Thas hint " $"$ in dem aller pesten selhlaff ermort; F. $440 \nabla^{\circ}$ ) er hub sich auf, dass mon in weder spurt noch bort, anf das sich die rndat allein auf die gedreuen finde.

\section{VIII}

Tles morgens man dass hindt ermordet fande.

ler morder sanmpt sich nit vud sprach zw hande mit falscher elag sein pruder das, tnd sprach: „Hab dir der lone ron der, die dw dort ron dem dot erlnste, der dw so vil nachlie gedrawet haste.

Nit nichte dirs abpitten lass! Schaff, das mon ir dw ane den aller pesten dot anleg vmb willen irer falschen ptleg! ‘ 
Der her sprach: „Fruder meine, an irem leibe mon sie nit pedricben sol, wan in ein wilde insel so wil ich sie wol

vervuren. Las, das sie vur was mein ingedenck dw seine!“

\section{IX}

Der graff gab sie eim schiffinon vad gepotte, das er sie in ein insel seczt on notte;

der anf dem mere durch ir schön seinss willenss ron ir geret.

Doch halfi in wider gutt wort noch falsch pette, sunder sie antwurt gutlich durch ir stette:

„Lass ab ılein clag vnd falsch gedön. wan dw wirst nit geweret;

e wil ich sterben in der wilt.“

Zw hant seczt er das schone pilt anf ein fels mit geferdte

rud fur vou dan. Do schrey die edel keisserin:

„Kum mir zw hilff, dw himeliscle kunigin!

Dein hilff mocht mich wol machen kön ron meiner grossen sthwerdte."

\section{X}

In solcher angst rnd herczenlieber schwerde wart sje ror leit hinsincken zw der erde vnd schliff. In dem draum ir furkam Maria, die vil rejue, rnd sprach: „Ich wil dich leren zw der stunde, das dw die sundersichen machst gesunde mit einer woreze lobesam.

Darvmb do grab palt eine rnder dein hanyt; die hat solch kirafft: wer mit dem alssacz ist pehafft, der wirt darvon verlone."

Da sie erwacht, die wurc grub sie pelendiklich.

In dess ging her ein schift mit gut; des freit sie sich.

In dess sass sie rnd vur gen Ram; gab oftlich zil verstane, 
wie sit den anssac\% genezlich kunt verlreiben mit gottes hilft peid mancen vond dem weiben an all leipliche schwerikeit.

Non in der zeit gar hattr dess keiserss pruder, der sie het versaget, mit dem aussarz gar sehwerlichen geplaget.

lir sprach: „O) we, meinss grossenss leit!

Wan kumpt mir die rudatte?*

Des gleieh dess graften pruder was ansergig in solyeher mas.

Der gratf fult in gesehwinde gen Raıı. „(H) mon in dnrelı gross kuust möcht machen rein. mit grossem schacz solt disser arczt pegabet sein," also liess er in sunderheit zw liom oflich verkunde.

\section{XII}

Dess kam gen hoff wol rir dem keisser liere, wy das ein fremde fraw herkmmen were, kmut solche schwer rerdreiben gar. Der keisser nach ir sente.

Dis edel ereztin kam gen hoff zw bande, dass sie $z w$ hoff doch nymant nit rekante.

ler k+isser sprach: „Fraw, nement war, went ron in al pehentte

soliche schwer. dem pruder mein!

llit einem man ganez selberein solt ir pegalet werden."

sic sprach: "Krims schacz noch guttess ich darrm peger, sunder dem hoclsten Got gib icb darin dy er, $\left(\right.$ F. $\left.441 \nabla^{0}\right)$ durch den jeh auch mein kunst nit spar:" Der keisser mit pegerden

\section{XIII}

nan palt sein pruder, furt in zw der frawen;

vol anch der graff rir sein pruder der schawen, ob in geholften wurt durch sy; 
das wolt er selb rerschalde.

Die fraw die stund vor sira rnd sprach: „Wolane!

Wolt ir reriehen liy ror ider mane.

was ir ye det icz oder ye,

ench wirt geholffen palde."

Dess keiser'ss pruder offenlich

ror allem rolek pekennet sich

al seiner missedate,

pis on allein das er ans lugemhaftem munt

die edlen keisserin falschlich versagen kunt;

das wolt er auch veriehen ny

durch falsch seins herezen ratte.

\section{XIV}

Solich rerhaltung mercket die vil gutte;

sie sprach: ,.Mit wissen nit rerschweigen dntte,

smuter euch rberwindet gar,

wolt ir anch werchen reine."

Der keisser sprach: „Pruder, wejstu noch mere

etwass, dass wider mich so groblich were,

das oftenbar hy vor der schar!

Hab forcht rnd sorgen cleine!"

Er fiel zw fuss dem keisser reich

Fnd det reriehen offenlejcb.

wie er die keisserine

gegen dem keisser felschlichen rerlogen het, darvmb dass sie nit wolt geweren seiner pett.

Do das der keisser wart gewar. pedript wurt im sein sine.

\section{XV}

Erst nam die fraw die wmrz rnd schneit sie cleine,

davon dess keissers pruder vil rureine

auch offenlich ZW drincken gab;

do wart er rein rnd elare.

Dess graffens pruder hub do an zw rlagen,

was er ye det jey allen seinen dagen, pis auf dass kiut; da lis el ab,

XIII, 6: Ms. vir. - XIII, 13: MS das edlen; kynt - XV, 5: do manque dans le ms. 
Ind schweigsam bett ers gare.

(F. $442 \mathrm{r}^{n}$

Die fraue sprach: ,Tersucht euch pas,

rnd wist ir mer, so oflinet das!

Jurch nichte dass nit late!"

luss kindes mort er ast do oftenlich reriach.

l) frew nit irem dranek der wurcz sie in versach, als ich das gschicht gelessen halh.

Jal wart er rein gar dratte.

\section{XTI}

tross lob gesproduen wat dem zartten weibe, dass sie so palt macht rein der zweyer leibe

an alle schwer mit irer knust;

dess wundert menikleichen.

Doch was noch ruerkant die zarte frawe;

do nent sie sich vul sprach zu im: „Nun schawe:

('ur falscless werben wass rmbsmst;

mein er philt ich genezleichen."

Itlicher gnad von ir pegert;

zw fuss ril ir der keisser wert, schon erlich sie empfinge.

Der keisser liss ein hoff ansriffen ir zW er.

I le fraw die sprach: ,Kein mon perurt mich nymer mer;

mein herez in hiezelicher prunst

pegert geistlicher dinge."

\section{XIII}

Nach ir pegirde ir der keisser reiche peleit in ein kloster demntikleiche.

Tarin erlanget sie von Got die kron der erikeitte.

.Daran gedenck ('in reines weib: der eren mit nichte solt dein elich man rerkeren,

rnd halt ess alss dir Got gepot;

jiss auch nit so verzeitte!

Wan $d w$ schon angelanget werst, gedenck, das dw in widerferst, rud phatt dein weiplich krone,

XVI, 14: Ms. hiczliher. 
die dir so hoch rber al krou erhaben ist", spricht Albrecht Paumbolez, pittet Got, den waren ('rist, das er rns in der lecten not

(F. $\left.442 \nabla^{\circ}\right)$ welle allen pey pestane.

\section{$\mathbf{N}$}

\section{Der Teichner}

(Ms. Dresde, Kgl. öff. Bibl., M 203, pp. 47 b -53 a).

Die Rede hat yemacht der Teycher.

(Pag. 47 b)
In der Römer puch man las,

Das ein gewaltiger ehnnig was;

Der must in ein herfart.

Do stund allez sein gepart,

s Wem er liez sein frawen pider,

Unz er chem zu land berwidere.

Do wart im geraten, wie

Daz ers seinem pruder lie:

Jer behielt ims wol mit er'u.

10 I'amit so must er damen chern.

Als pald rucl er rait sein strazz.

to verzaigt der pruder daz,

Daz er was ein pöser man

Tnd anch sucht die frawen an,

15 baz si seinen willen tät.

Do veriach die lrawe stät:

"Waz wolzt an dir selher rechen? (Pag. 48 b)

Wolzt $d u$ dein trew am prider

$$
\text { prechen? }
$$

ob ich denn so pös mu wär

20 Vnd don deinen willen pär,

so gehört es dich nicht an."

Do sprach er: "Es mïz ergan,

Stunden bundert trew ze phant."

Do sbrach die fraw zehant:

25. "Seind es nicht anders wesen mag,
So rernim, waz ich dir sag:

So haiz ein haymleich wesen pawen,

Daz daz niemand mag gesihawell,

Daz der sünden rnd der schant

30 Nempt werd innen in dem lint."

Daz geschach, er pawt ein rest

An ein heimleich ort, die er wol west.

No die vest volchomen sehain,

Do sprach die frawe rain:

3.; ,. Noeh ist nicht gewerens zeit.

Ir schawt, wo ezzen rod trinkchen leit,

1)az wir die rest beriisten gar

Tollichleichen auff zway jar:

Ezzen vud trinkehen miizz wir han."

40 Er sprach: "Daz ist schir getan."

bo sprach er zu der guten:

"Du solt nicht lenger hrides muten."

Si sprach: „Ja, die zeit ist hie."

Vor die frawe in lie vest er gie;

4.5 sie verspert in mit dem tor:

Er belaib drinn, si was hiesor.

Also must er sein virspart,

Ynz daz sich endt die herfart.

tho der herr 110 cham herwider,

5n Auff lub sich die trawe pider,

XVII, 15: Ms. pey sey pestane.

27. Ms. Sr(?) haiz. 
An die tiir sie sigt im mer,

IVie ir lerre eliültig wïr;

site sprath: ... lch merkeh anch rond spür,

laz dn gorne werst herfür:

sis so will ich dieh lazzon frey.

siprich, das ieh an sorgen sey!“

10 gab er it trew rnd ayd,

Er gewünseht ir nymmer layd,

I haz er ir hernach zebrach.

6o Als pald rnd er den pruder sach,

All sein trewe die was do ab,

Daz er die frawen schuldig gab):

(Yag. 49 a) sie hiet vels ril getan

Jit so manigem slatman.

6.5 Iha vertog (ir's also scharti,

Inz mans in die Teyfor warfl.

Io cham si in ein viselıpant,

Inz sey cin rischer zorh ans lant.

larmate chom die frawe gat

7n Norh in grozzer aremut,

Ihaz ein herzog nach ir sant

Find sich der frawen ruderwant

Mit also rehten trewen gut,

Dar si iur eins chindleins hut,

75 Daz (2) ir in trewen enphalieh.

to was an dem hof ein walich.

ler des fürten diener hiez.

ber het neyd and widndries

Hinz der frawe ane schuld.

so Lamit si anch verlos sein buld,

Daz er begert prey ir ye ligen,

Tud do der pös des wart rerzigen,

Do stumd stets aller sein Heiz,

Wie or ses pracht in ytweiz.

xs In ein chamer el chömen plulag,

Ha die fraw rudtz chind da law.

(Pag. 49 b) Mit d'ns swert daz chind er sterhet, Als es die lina hiet verderbet.

Er sagt dem fin'sten rul hofyesind.

su Die fiaw wer sthullig an dem elind,

Des sey liot visclunldig chant.
Lo veriaelı der fïrst zehant

Zu dem poswicht: „Ruht hinz ir!

Wa\% du un wild, daz ist mein gir."

95 1) si dem poswicht wart geben;

Do gewans nie vbler leben.

Wiz er marter mocht erdenkrhen,

Hanit tet er sey bechrenkcher.

Also slaift ers anf und nider.

110. Zelezt in die Teyfer wider;

la si ror was anzenomen,

Also musts hinwider chomen.

Joch was der lieb Got ir trost,

Als der yr die seinn erlost.

10.5 Do si in dem wazzer ran.

1) ler plikehts ein alter man;

Der sprach: ..bing aus dem wag,

tinte fraw, and nicht verzag!

Ich bin es, Peter. Gotes pot.

$110^{\circ}$ Dich hat nie verlazzen Got

(Pag. 50 a)

Ynd wil dich halt nicbt verlan.

Ge rud nim dich ermey an

In die stat der Rümer sehar!

Ind wer dir peichtet offenhar,

115 Waz er sünden hat getan.

Jer wirt ein gesmnter man

Vud stet anft mit Gotes gewalt,

Wie sein siechtung ist gestalt."

1)o die Rümer daz erfrieschen,

120 Nach der frawen sie do yeschen.

Do war hesundersiech ir man

Fnd sein pruder, der vortan.

Vnd der man des herzogen,

ler sey anch het rerlogen.

12: lie sey hetten ze töten gemainet.

Die wurden alle drey gererainet

Mit der smdersiechen pein,

Daz doch nieht swachers mocht

$$
\text { gesein }
$$

Vub daz jemerleich verraten,

${ }_{336}$ )atz si an der frawen tilten.

baz het liot hinz in gocochen.

Waz si hetten anfi sew gesprochen. 
Das sie nicht gdacht noch gerüret.

Also wart die fraw gefüret

(Pag. 50 b) 135 Für irn rechten ehemanu,

Daz si in legte erzney an.

si chant in wol, er chant ir nicht.

Do sprach die frawe gericht:

"Es ist alle erzney verlorn,

$1 \neq 0 \mathrm{Ir}$ mïzzt peichtig werdı rorm.

Got hat mir die gnad gegebn,

Wer mir peichtet alz sein leben,

Der wirt ein gesunter man,

Wie sein siechtung ist getan.

145 Anch sein peicht sol offen sein,

șo er lawest mag geschrein,

Daz mans vberal gehörn chan."

Do verschrakcht der herr dervan,

Iaz er offemnlich solt peichten

150 Vnd sich selb so gar verleichten.

Er sprach: „Liebe frawe zart,

Wer ich damit wol bewart,

Ob ich heymlich peicht ein tail?*

Si sprach: „Nain, ez macht nicht

hail."

155 Also sagt er sein gewissen

Ottenbar rnd vurerizzen,

Dann daz ain dawcht in ze scharff,

(Pag. 51 a) I) ma man die frawe ind Teyfer wartf;

Daz wär leicht vor scham verswigen.

160 Doch liez es die frawe nicht ligen;

Si sprach: „Herr, bedenkt ew paz:

Ir habt rergezzen etzwaz.

Wer mir ganz veriehen tut,

Der ist zehant gesunt rudjfrut

165 Also versten ich mich dabey,

Inaz ewr peicht getailet sey.

lavon tut mir anff die tür

Vnd lat ez allez samt herfuir,

TVelt ir gesunten leib hie schawen."

170 Also peycht er vor der frawen,

Die sein hawsfraw was rnd hiez,

Wie er die rerderben liez.

Alsin drat er des veriach.
Verswunden was sein vngemach,

175 Vind stund auff an allen pein

Ind gie zum siechen pruder sein.

Er sprach: „Lieber pruder, sich!

Aller deiner siunden gich

Der frawen offennleichen hie,

${ }_{1 \times 0}$ so wirstn gesunt als ye.“

I) verschraket er von den pünden,

(Pag. 51 b) Daz er offenleich solt chïnden.

Doch pracht in der pruder dran,

Daz er lub die peychte an

185 Ind sprach $5 n z$ an die stat,

l)az er mit seinem falschen rat

Het dem pruder daz weib rerworn;

Do geswayg er vud lag als vorn.

Do sprach die frawe geslecht:

${ }_{190}$, Ewr peycht ist nicht gerecht.

Hiet ir recht gepeicht von grunt,

So stïnt ir anf vnd wert gesunt.

Wer mir genzleich tut veriehen.

Ier ist mit der vart gerehen.

${ }_{195}$ Davon sult ir nichtz versparn;

Ir sult es alles oftenbarn,

Welt ir haben gesunten leib."

Do gedacht er des pruder weib,

Diz er peychtig wurd dervan,

2an Maz er ir rbels hiet getan.

Alsen drat el sich des erchant.

Do was er anch gesunt zehant

Vnd stund anf an allen schaden.

Darnach so wart die frawe geladen

205 Hinz der herzoginne man,

(Pag. 52 a) Der ir ril laides hett getan

Tnd begert nu hilff an sey.

Do sprach die wandelsfrey:

„Ewr sjechtung ist also getan,

210 Das ew niempt gehelfen chan,

Ir mïzt offemmleichen sagen

All ewr siind von chindestagen."

Do sprach der sündig man:

"Wer het ye gehört daran,

215 Daz mau oftennleich sol peychten!" 
J) sprach si zu dem leicliten:

.. D ${ }_{i j}$ ist anders nichtes an:

Welt ir werden ein gesunter man.

Ir müzt offemnleichen iehen.

220 Die weil ewr peycht ist vngereben,

so seyt ir auch des chrmmpen leibes."

Do volgt er dem rat les weylues.

Daz er pejeht auff alle ort

Von dem zwivaltigen mort.

225 Wie er des herren ehind erstach

Vnd das an der frawen rach.

Als pald rud er daz erschainet,

Do was in anch sein leib gerainet.

Do die drey wurden berait

(Pag. 52 b) 230 Vos der möselsüchtichait,

Die zwen an eren wurden tot,

Daz ins niemand wol derpot.

Jo sprach manig man dapey:

„Man solt sein verderben drey

$235 \mathrm{Vmb}$ die ainige posheit!“

Des geuieln si auch in arbeit,

Daz si der fürste slayffen liez

Vnd in die Teyfer werfien hiez,

Als si teten der frawen rain.

2*0 Alzo geschach in allen zwain,

Daz man sie möselsüichtig rant,

Tud aucl sagen musten ir sehant

Gar ottenleich vor layn vnd phaffen lis war uoch hewt ein edelz straften.

245 Wer die lent mit liggen beschwaehet,

Daz den Got zu nichte machet

Mit den sunderleichen maylen,

Jaz den niemand mocht gehaylen;

Lir must offenleichen ichen,

250 Waz im sünden wär geschehen,

Oder waz er liegens ye hiet phlogen:

So wurde dester myn gelogen.

Doch in lezten Got daz wolt,

(Pag. 53 a) Daz der herr erchemnen solt,

255 Daz die ärztinn wär sein weib,

Die in da macht gesunten leib.

Darnach lebt der herre guet

Vnd die frawe gar wolgemut

In der werlt, daz ist wat, 260 Sagt rns die schrifft, zway jar

Vnd leicht ettlich tag hintmr,

Daz sie rolgten der natmr.

Darnach lebten si geistleich,

Daz si verdienten daz himelreich:

265 Er wart ein mönch, si ein nonn,

Auz der falschen werlt entronu

Zu den frewden vnwandelber.

Also sprach anch der Teyehner.

Also hat das puch ein ende.

270 Got behüt uns ror missewende! 


\section{E R R A T A}

P. 25, n. 1, 1. 1: $82 \mathrm{v}^{0}$, lisez: $82 \mathrm{r}^{0}$.

P. 26, n. 3, l. 3 d'en bas: said, lisez: said.

P. 36, 1. 6: d'un, lisez: d'ung.

P. 65, n. 3, l. 2: u der, lisez: u. der. 


\section{TABLE DES MATIĖRES}

('hapitre I. ('lassement gènéral des diffẻrentes rersions

('hapitre JI. Origine du conte 3

('hapitre III. Tersions orientales

('hapitre IV. Les Gesta Romanorum . . . . . . . . . . . . . . . . . . . . 23

Chapitre Y. Florence de Rome . . . . . . . . . . . . . . . . . . . . . . 28

Chapitre VI. Le Miracle de la Vierge . . . . . . . . . . . . . . . . . . . 32

('hapitre VII. C'rescential . . . . . . . . . . . . . . . . . . . . . . 60 60

('hapitre VIII. Hildegarde . . . . . . . . . . . . . . . . . . . . . . . 65

Cbapitre IX. Conclusion . . . . . . . . . . . . . . . . . . . . . . . . 80

('hapitre X. Table alphabetique des rersions . . . . . . . . . . . . . . . . . 8 8

\section{Appendice:}

A. Version du Manse-Buch, ed. de Wilmersdorf (traduction) . . . . . . . . . . . . 97

B. Tersion d'Al Faray Bu'da Alsidda, ms. Londres, Brit. Mus. Or. 237 (traduction) . . 99

C. Fersion des Gesta Romanorum anglo-latins, ms. Londres, Brit. Mus. Harl. 2270 . . 111

D. Version latine en prose du Viracle de la Vierge, ms. Paris, Bibl. nat., f. lat. 14463 . 116

E. Version latine en prose du Viraele de la Vicrge, ms. Londres, Brit. Mus. Harl. 2316 . 120

F. Version latine en rers du Miracle de la Tierge, ms. Paris, Bibl. nat., f. lat. 17491 . . 122

G. Version française en prose du Miracle de la Tierge, ms. Paris, Bibl. uat.. f. fr. 1505 . 129

н. Version des Faiz et miracles de Nostre Dame, ins. Paris, Bibl. nat., f. fr. 410 . . . 132

I. Version fringaise en rers dn Miracle de la Vierge, ms. Paris, Ars. 3516 . . . . . 134

J. Version de l'Alphatietum narrationum d'Étienne de Besançon, ms. Paris, Bibl. nat.,

f. lat. 15913 . . . . . . . . . . . . . . . . . . . . . . 149

K. Version de la Stella maris de Miraculis Beate Marie Virginis de Jean de Garlande, m.. Londres, Brit. Mns., Roy. S. C. $\mathrm{N}^{\top}$. . . . . . . . . . . . . . . . 150

L. Version de la Tie des Pères, ms. Paris, Bibl. nat., f. fr. 1546 . . . . . . . . . 151

M. Version dAlbrecht Baumholtz, ms. Berlin, Kgl. Bibl., Cod. Germ. 414 . . . . . . 161

N. Version de Der Teichner, 1ns. Dresde, Kigl, öff. Bibl., II 203 . . . . . . . . . 169

Errata . . . . . . . . . . . . . . . . . . . 173 



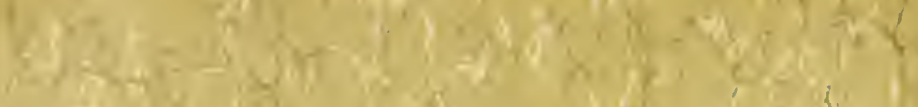

7. $4 \times 28$

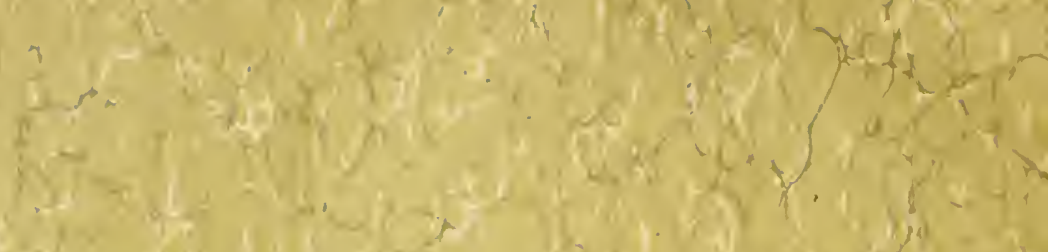

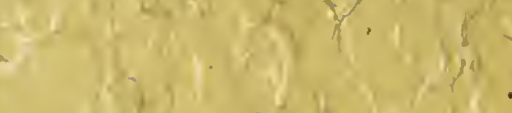

$y^{2}+x^{2}+y_{0}$

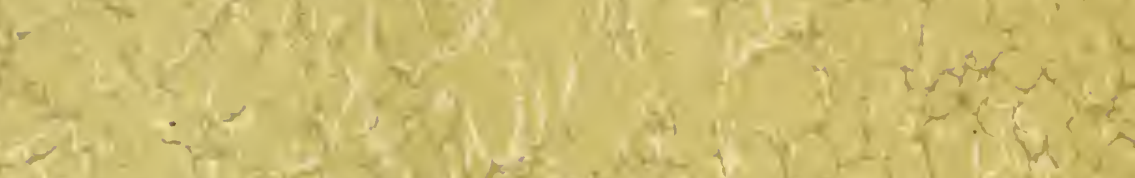

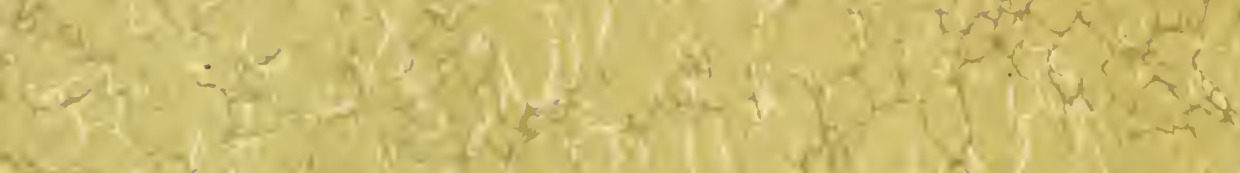

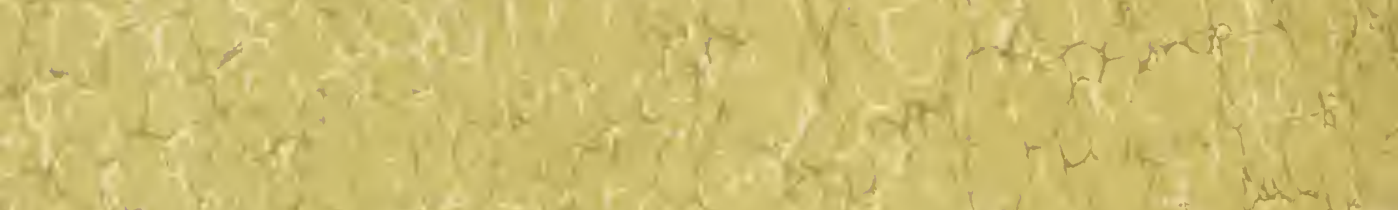

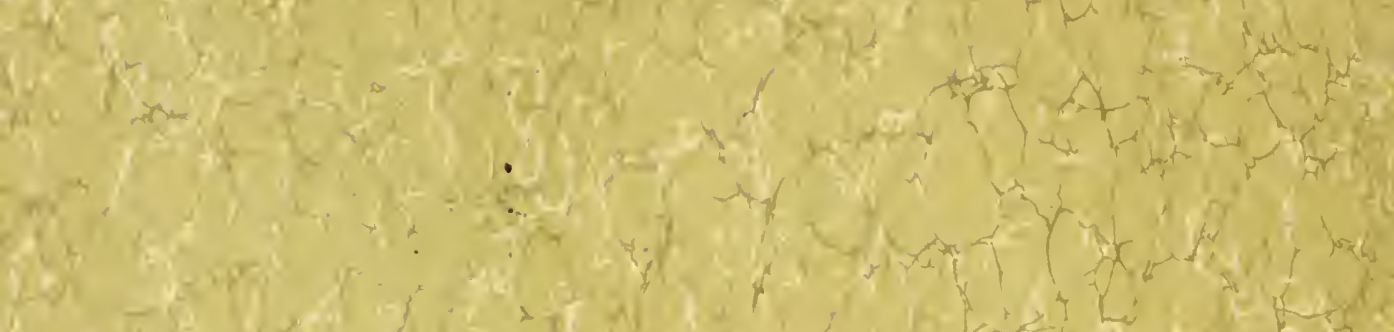

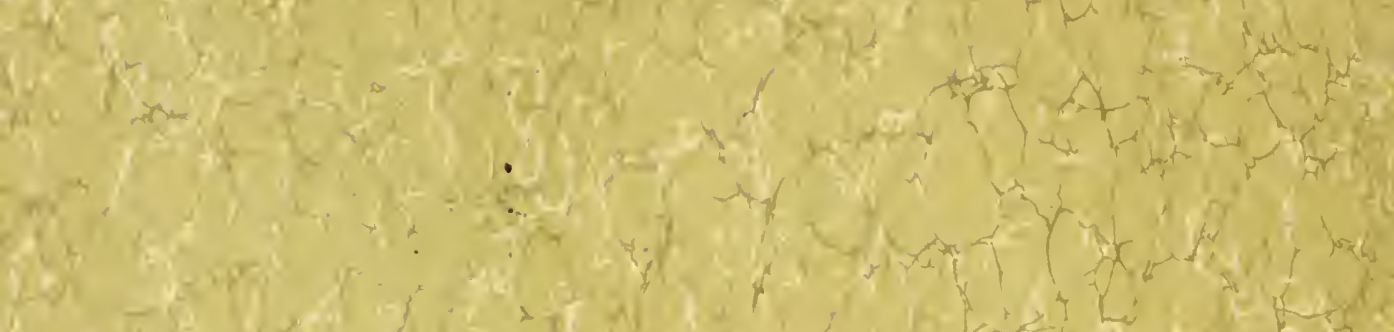

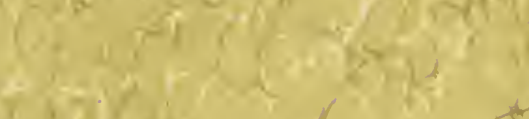

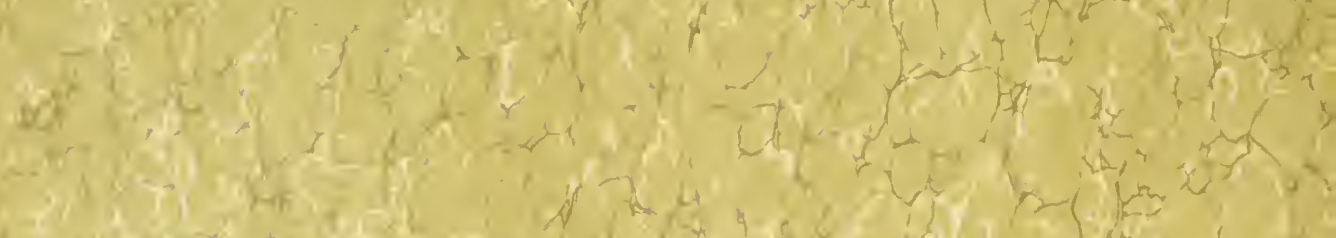
(a) tis

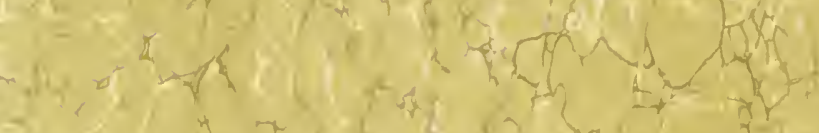

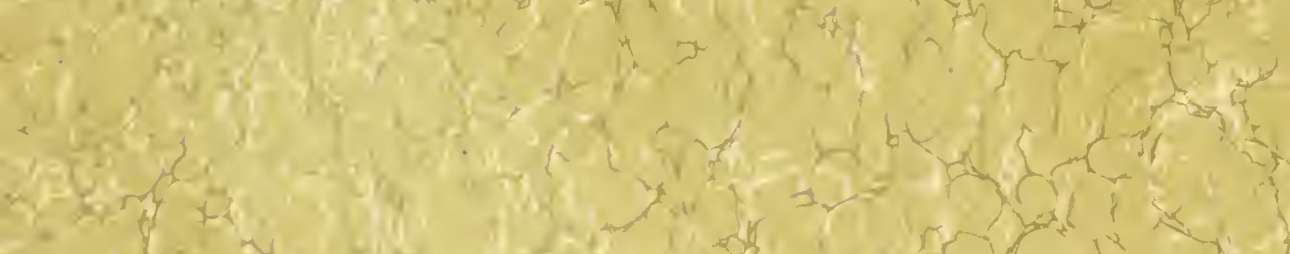
50 a de

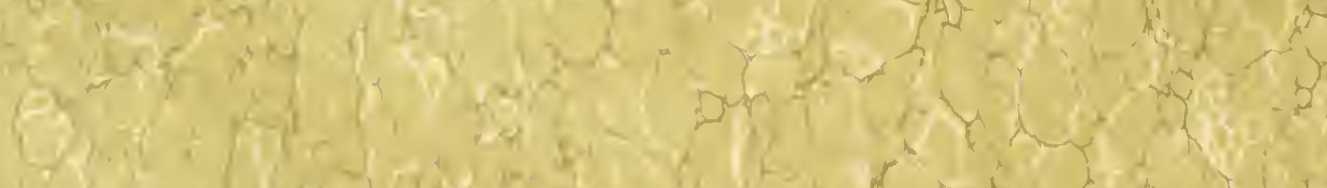
4. 


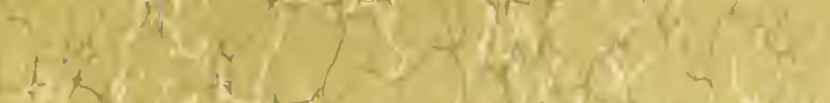

(x) zed at

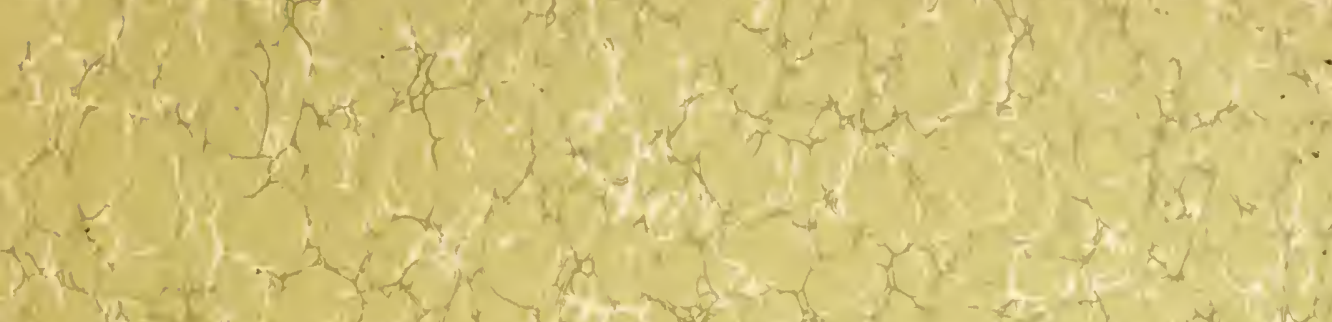

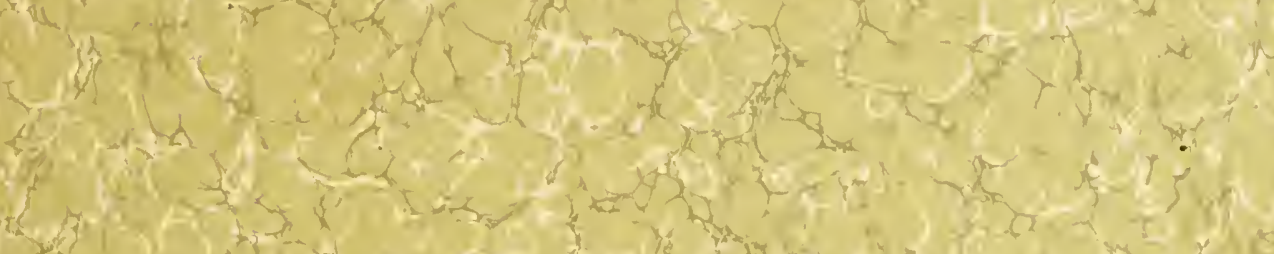

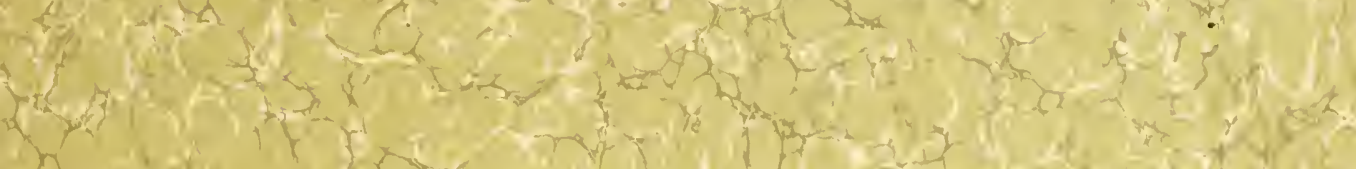

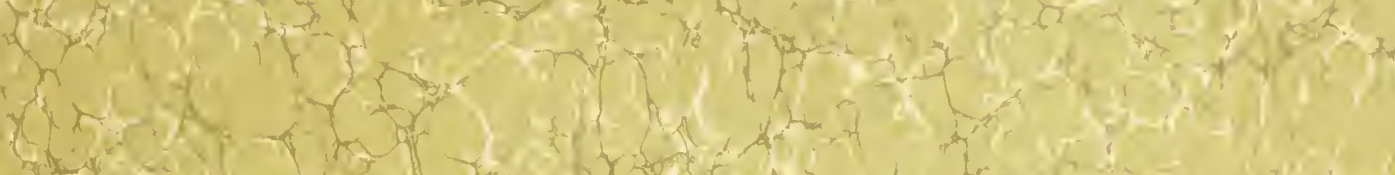

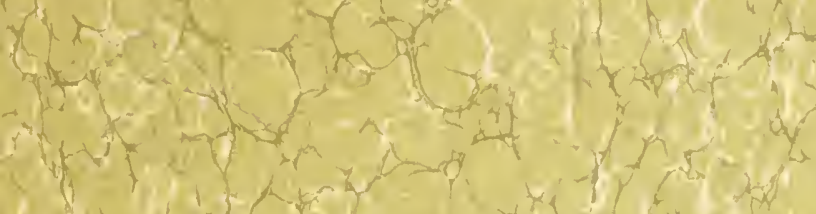
(1) 
